\title{
A novel high-throughput genomic screening tool in Saccharomyces cerevisiae Namal Coorey
}

A thesis submitted to Victoria University of Wellington in part fulfilment of the requirement for the degree of Masters of Biomedical Science 


\section{$\underline{\text { Abstract }}$}

The elucidation of drug targets and their biological effects can be aided by the identification of yeast deletion mutants that confer hypersensitivity to the drug. However, the biological activities of some compounds are reduced by the mechanisms of the pleotropic drug resistance (PDR) network. For this reason a PDR-deficient strain with deletions in the master transcriptional regulators of the PDR network, PDRI and PDR3 was created. This double deletion mutant strain was robotically mass mated against the non-essential deletion mutant array (DMA) to create genome wide nonessential PDR-deficient DMA (PD-DMA). No phenotypic enhancements were observed with $\Delta p d r 1 \Delta p d r 3$ double mutant and the various deletion strains of the DMA.

Increased genome-wide sensitivity of the PDR-deficient mutants was demonstrated by screening pools of PD-DMA and the DMA against cycloheximide, a Pdr5p substrate and rapamycin which is not. Similar sensitivities were observed for the non-PDR substrate rapamycin for the two deletion mutant pools while sensitivity to Pdr5p substrate cycloheximide was significantly more sensitive in the PD-DMA.

The genome-wide increased sensitivity of the PDR-deficient mutants was further assessed by screening the LOPAC library of pharmacologically active compounds against pools of PD-DMA and the wild-type background DMA. The DMA screen identified 5 of 1280 compounds having bioactivity whilst the PD-DMA screen identified 25 compounds including the 5 identified by the DMA. The 20 additional compounds identified in the PDR-deficient background were inactive at the concentrations used in the wild-type background.

The PD-DMA was then used in chemical genetic profiling assays; namely solid-phase chemical genetic profiling and DNA barcode microarray experiments. The PD-DMA was screened against natural products, rapamycin and cycloheximide with well characterized chemical genetic profiles. The PDR-deficient strains hypersensitivity to rapamycin and cycloheximide were indicative of TORC1 pathway inhibition and translational elongation inhibition, respectively. This was consistent with literature as rapamycin is an inhibitor of TORC1 and it mimics nutrient starvation response and cycloheximide inhibits eukaryotic translational elongation. These results validated the 
utility of PD-DMA as a hypersensitive genome-wide deletion reagent for chemical genetic profiling.

Following validation of the PD-DMA, biochemical assays were performed on latrunculin-A, a less well characterised marine natural product and Plakortide-T a marine natural product with novel activity. These inhibitory drugs were shown to be PDR substrates with biological activity at low nanomolar concentrations. LatrunculinA, was $\sim 28$ fold more potent in the PDR-deficient strain. In contrast, plakortide-T was biologically active only in the PDR-deficient background and the PDR mediated efflux did not involve the major efflux transporters.

DNA barcode microarray experiments performed with latrunculin-A identified several hypersensitive deletion mutants consistent with cytoskeletal disruption specifically, actin microfilament assembly. Latrunculin-A is known to bind monomeric G-actin and inhibit actin polymerisation. However, in novel findings tubulin cytoskeleton disassembly was also shown to be mediated by latrunculin-A.

Plakortide-T belongs to a class of compounds that disrupts calcium homeostasis. DNA barcode microarray experiments performed identified 56 deletion mutants hypersensitive to Plakortide-T, however, none were involved in calcium homeostasis and the deletion mutants were not over represented in any of the GO terms. Plakortide$\mathrm{T}$ caused hypersensitivity in several deletion mutants of genes encoding for mitochondrial proteins. This activity however, did not generate reactive oxygen species as increased oxidation of free thiols or induction of the oxidative stress response was not observed. Plakortide-T was shown to induce an increase in cytosolic calcium detected by the nuclear localisation of Crz1p, a transcription factor activated in response to increased cytosolic calcium. This activation was dependent on functional calcineurin which further validates this response is an increase in cytosolic calcium. 


\section{Acknowledgements}

I would like to begin by thanking my parents. I would not be here if not for you. Thank you for putting up with me through the ordeal that was the Master's degree. To my mum for making sure that I was having my meals when working on the thesis was all I seemed to do and my dad for all your encouragement and for constantly reminding me that I am almost 25 of age and have not yet completed the master's degree. To Gabriella my love, for sticking with me through the weekends on at end (for almost two years) that I spent working in the lab rather than spending time with you. I can finally take a small break now.

I would also like to thank other member in the Chemical Genetic Laboratory specially, Dr. David Maass, Dr, James Matthews, Dr. David Bellows, Rosemary Heathcott, Bede Busby, Christina Roberts, Liam Sampson and Peter Bircham for all you help and all the other members both past and present for making the past two years memorable. We have had our moments.

On a more serious note, I would also like to thank my supervisor Prof. Paul Atkinson for all your input and support, and for taking me on as your student although you already had more than double the number of students as other supervisors.

Ploi, it's never too late you are only a plane ride away. Yee, you cannot lose weight without gaining muscle from exercise; and Peter, you are a perve not a tree. No amount of leaves are going to cover it up. Bede, Big car, Big gun, Big bike, Big maine coon cat... hopefully people will get the message. Liam, it was good to have a partner in crime when all the deadlines for assignments and write-ups were looming.

That's enough about the good times. Here begins my Master's thesis. 


\section{Table of Contents}

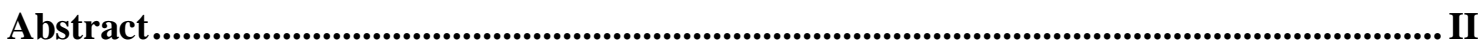

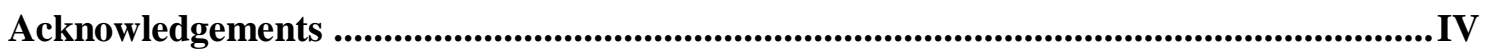

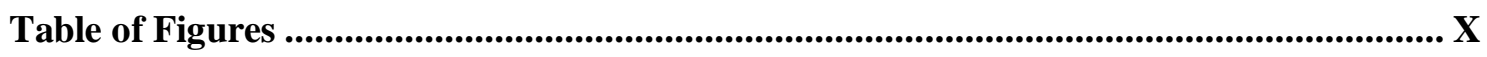

Table of Tables ........................................................................................................................... XII

List of Abbreviations ............................................................................................................. XIII

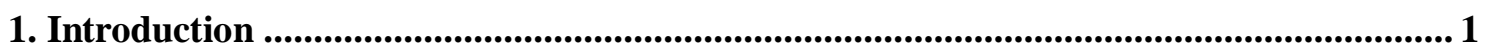

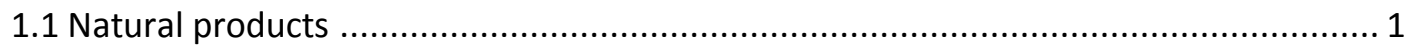

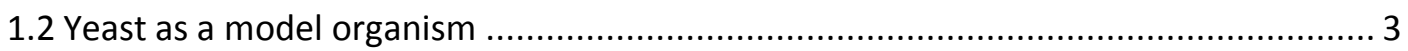

1.3 Various strategies for drug target elucidation and identification ................................ 6

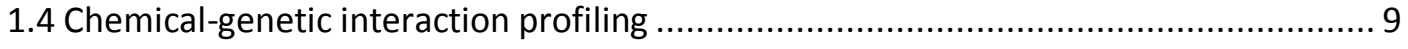

1.5 Chemical-genetic interaction profiles in yeast...................................................... 13

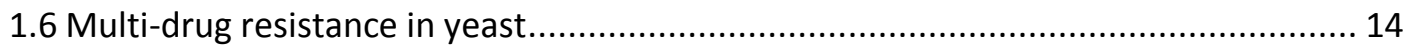

1.7 PDR1 and PDR3 are the master regulators of the PDR network ................................. 15

1.8 Differential regulation of PDR network by Pdr1p and Pdr3p ................................... 18

1.9 Chemical basis of substrate recognition by PDR pumps............................................ 19

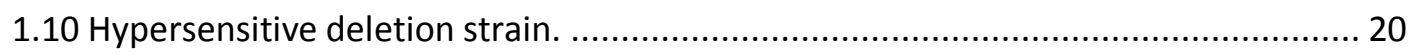

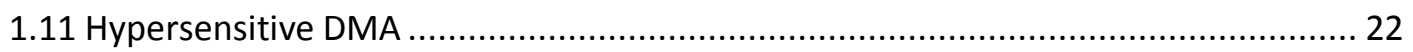

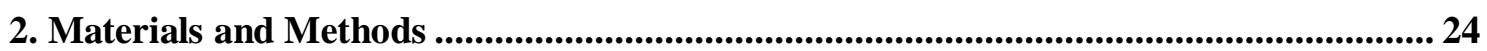

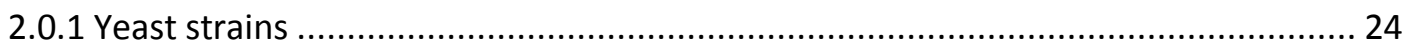

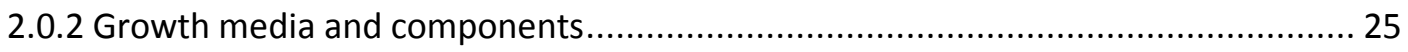

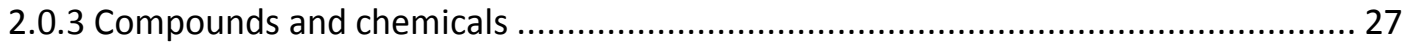

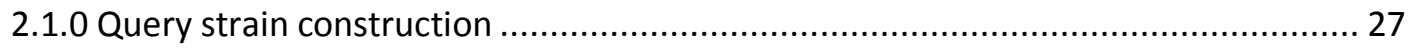

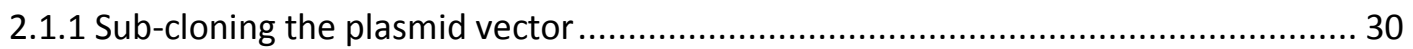

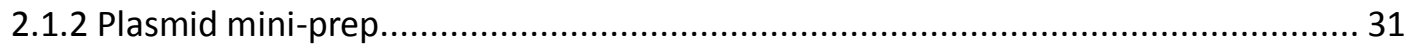

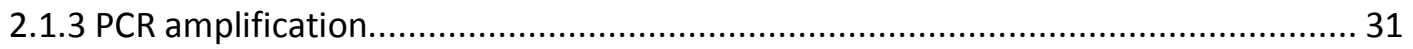

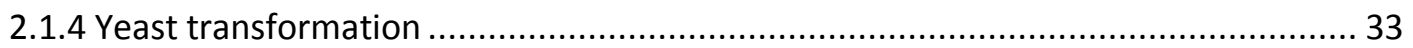

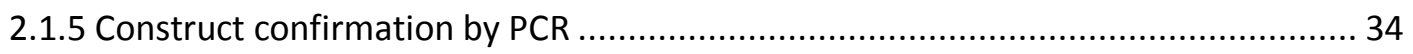

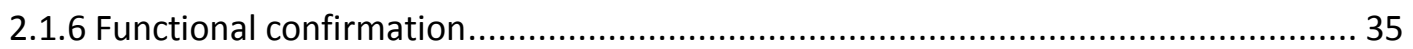

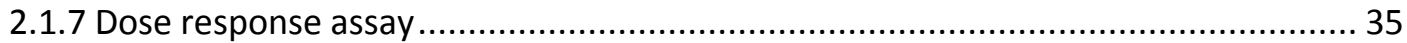

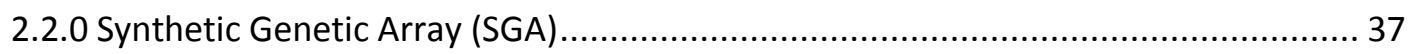

2.2.1 Synthetic Genetic Array methodology (see Fig. 2.3) ............................................ 37

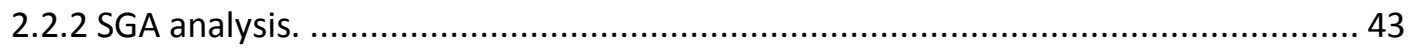

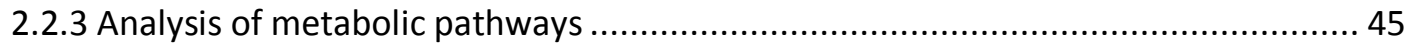

2.2.4 Confirming putative genetic interactions with $\Delta p d r 1 \Delta p d r 3$ double mutant............ 45 
2.2.5 Random spore analysis

2.2.6 Identifying mating and sporulation defects of single gene deletion mutants.

2.2.7 Storing PDR-deficient mutants for chemical genetic profiling experiments........... 47

2.3.0 Genome wide sensitisation of the deletion set to PDR substrates ....................... 48

2.3.1 Screening of the LOPAC library .......................................................... 48

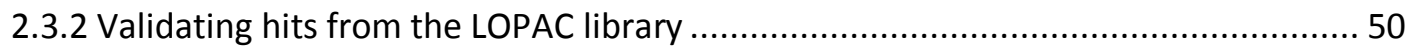

2.4.1 PD-DMA, media and compounds for chemical genetic profiling in solid phase ....... 50

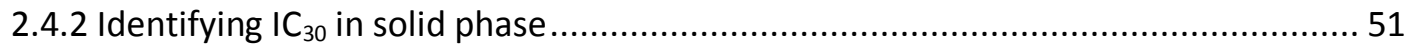

2.4.3 Chemical genetic profiling in solid phase............................................ 51

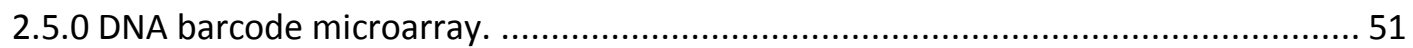

2.5.1 Gene deletion pools, media and compounds for DNA barcode microarray........... 52

2.5.2 Incubation of cells in compound-treated and control conditions.......................... 53

2.5.3 Genomic DNA purification ............................................................... 54

2.5.4 Quantitation of DNA with Hoechst 33258 dye ............................................. 55

2.5.5 PCR amplification of UP and DN tags with Cy dye labelled primers..................... 55

2.5.6 Precipitation of Cy dye labelled PCR products .......................................... 57

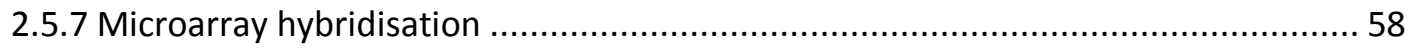

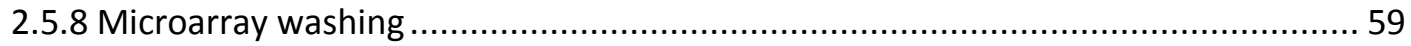

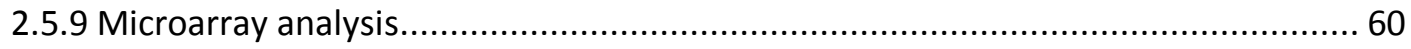

2.5.10 Validation of hits from microarrays........................................................ 61

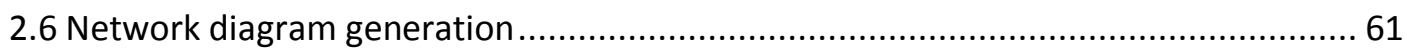

2.7.0 Image acquisition for confocal microscopy............................................ 62

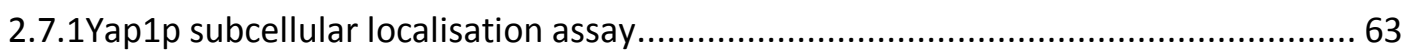

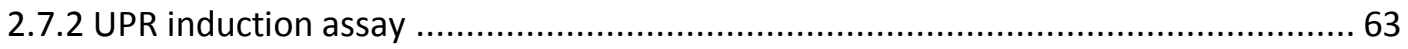

3. PD-DMA Construction and Validation. ...................................................................... 64

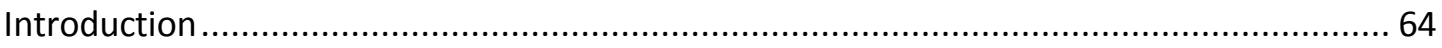

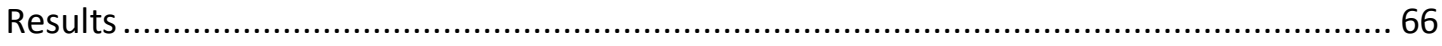

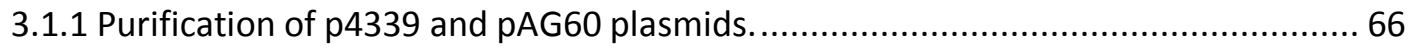

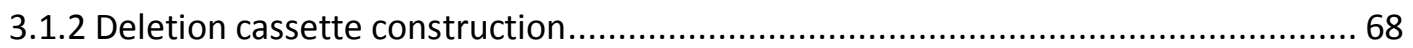

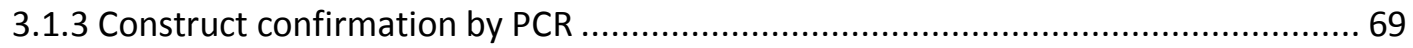

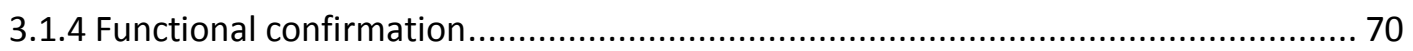

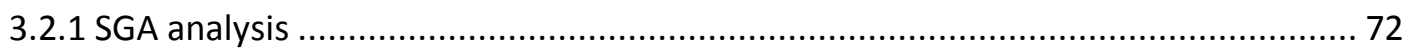

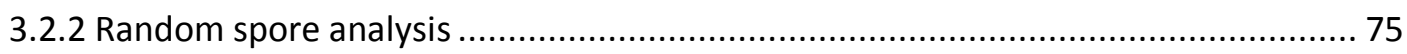


3.3 Loss of Pdr1p and Pdr3p transcription factors does not impair or induce the unfolded protein response (UPR)

3.4 Loss Pdr1p and Pdr3p transcription factors does not induce nor impair oxidative stress

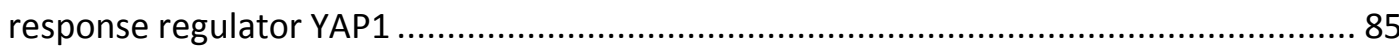

3.5 Genome-wide sensitisation of the deletion set to PDR substrates............................. 86

3.6 Deletion mutants hypersensitive to rapamycin ................................................... 88

3.7 Deletion mutants hypersensitive to cycloheximide ................................................ 91

3.8.1 The PDR-deficient mutants identify more bioactives from the LOPAC library. ........ 94

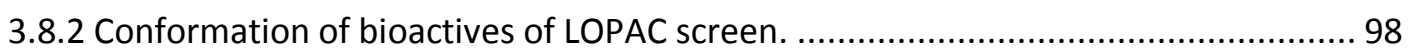

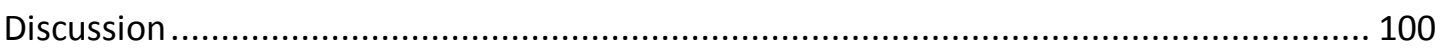

3.9 The $\Delta p d r 1 \Delta p d r 3$ construct and functional conformation.................................... 100

3.10 Loss of PDR1 and PDR3 does not activate oxidative stress response .................... 102

3.11 Loss of PDR1 and PDR3 does not induce or impair UPRE response....................... 105

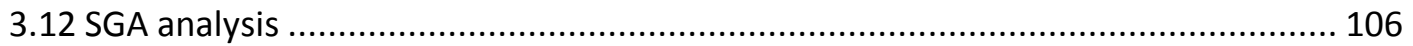

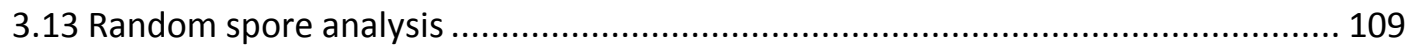

3.14 Deletion mutants sensitive to rapamycin ............................................................ 112

3.15 Deletion mutants hypersensitive to cycloheximide. .............................................. 114

3.16 PDR-deficient mutants identifies more biological active compounds .................... 118

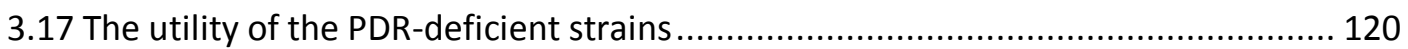

4. PD-DMA Validation Employing Latrunculin-A ....................................................... 121

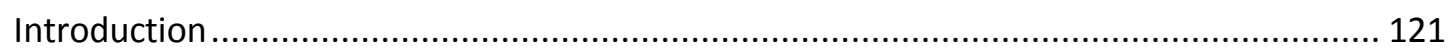

4.1 Marine natural products as lead compounds for drug discovery ............................ 121

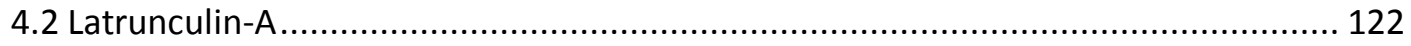

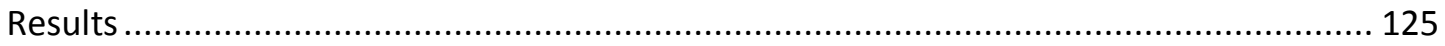

4.3 PDR-deficient strains are more sensitive to latrunculin-A.................................... 125

4.4 PDR5 is the major efflux transporter for latrunculin-A. .......................................... 126

4.5 Deletion mutants hypersensitive to latrunculin-A ................................................. 128

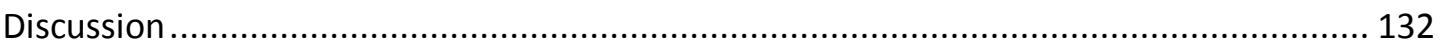

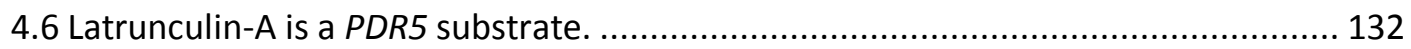

4.7 Deletion mutants hypersensitive to latrunculin-A .............................................. 132

5. Identifying Molecular Mechanism of Plakortide-T Using the PD-DMA....................... 137

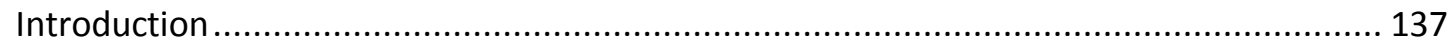

5.1.1 Plakortide-T, a novel marine natural product................................................. 137

5.1.2 Proposed mechanism of action for Plakortis metabolites ................................. 138

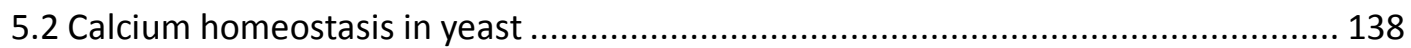


5.3 Disruption of $\mathrm{Ca}^{2+}$ homeostasis by reactive oxygen species (ROS) …...................... 140

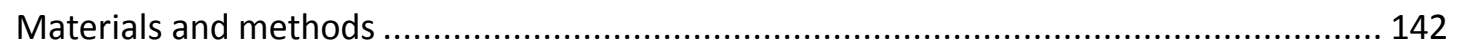

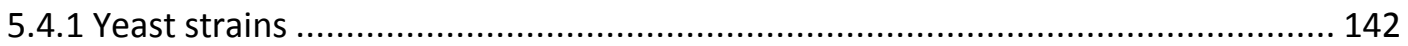

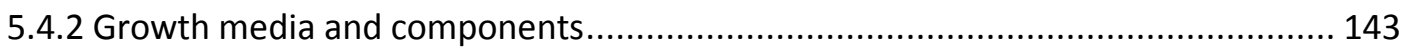

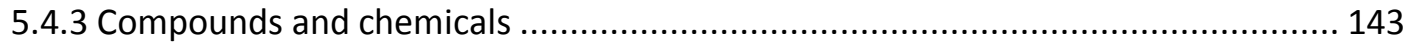

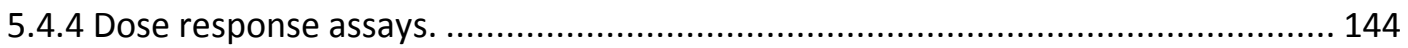

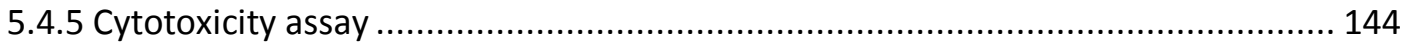

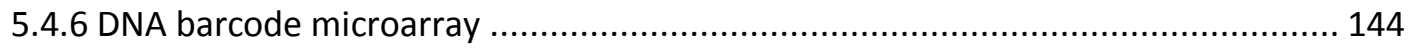

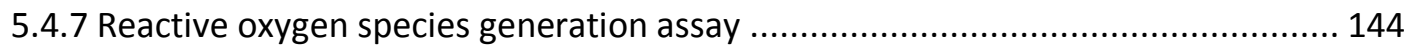

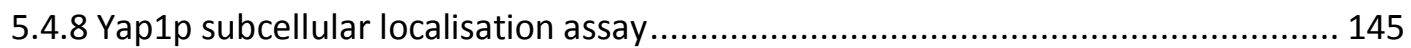

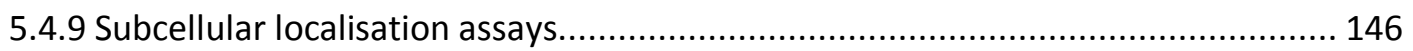

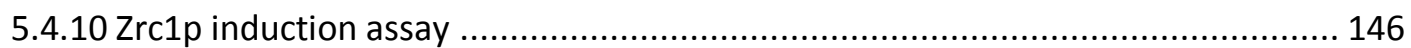

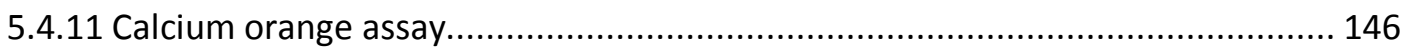

5.4.12 EGTA recue for plakortide-T induced growth arrest ........................................ 147

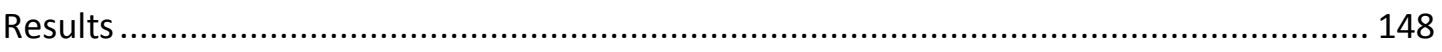

5.5 PDR-deficient strains are more sensitive to plakortide-T...................................... 148

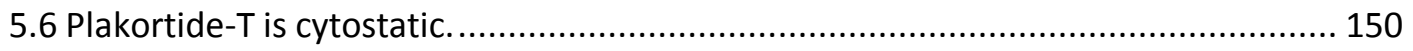

5.7 Plakortide-T is not a substrate for the major PDR efflux transporters ..................... 151

5.8 Addition of either $\mathrm{Ca}^{2+}$ or $\mathrm{Zn}^{2+}$ increases the potency of plakortide- $\mathrm{T}$ in a synergistic

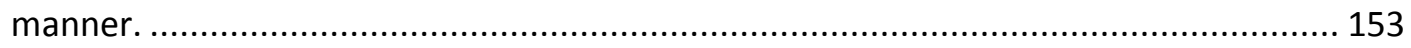

5.9 EGTA failed to rescue Plakortide-T induced growth inhibition ............................... 154

5.10 The sensitivities of the $\mathrm{Ca}^{2+}$ and $\mathrm{Zn}^{2+}$ transporter mutants and calcineurin mutants to

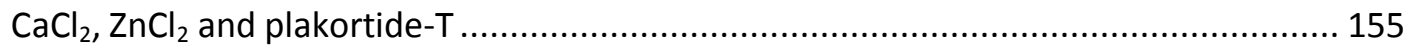

5.11 Deletion mutants hypersensitive to plakortide-T .............................................. 159

5.12 Plakortide-T does not induce the oxidative stress response regulator YAP1 .......... 162

5.13 Plakortide-T does not generate ROS detectable by DTNB ................................... 163

5.14 CRZ1-GFP as a reporter strain for increased intracellular $\mathrm{Ca}^{2+}$ levels...................... 165

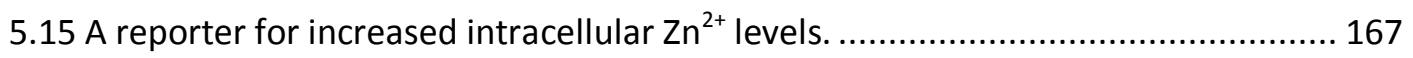

5.16 Plakortide-T induces either increased intracellular $\mathrm{Ca}^{2+}$ or $\mathrm{Zn}^{2+}$ or both................. 170

5.17 Location of Crz1p-GFP in $\mathrm{Ca}^{2+}$ and $\mathrm{Zn}^{2+}$ homeostasis mutants in response to

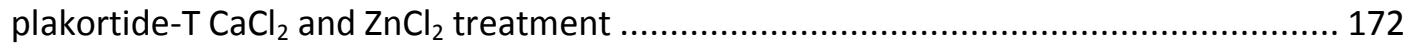

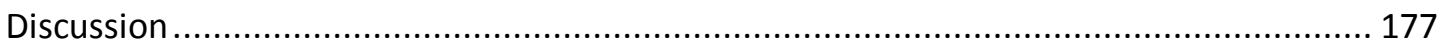

5.18 Plakortide-T is a substrate for the PDR drug efflux pumps................................. 177

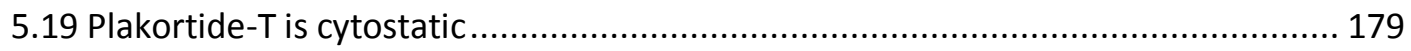

5.20 Plakortide-T effects can be exacerbated by $\mathrm{ZnCl}_{2}$ and $\mathrm{CaCl}_{2} \ldots \ldots \ldots \ldots \ldots \ldots \ldots \ldots \ldots . . . . . . . . . . . . . . .180$ 
5.21 Plakortide-T induced growth arrest could not be rescued by pre-incubating in EGTA

5.22 Mutants in $\mathrm{Ca}^{2+}$ and $\mathrm{Zn}^{2+}$ transporters and calcineurin do not show increased sensitivity to plakortide-T.

5.23 Deletion mutants hypersensitive to plakortide-T are not associated with calcium homeostasis disruption.

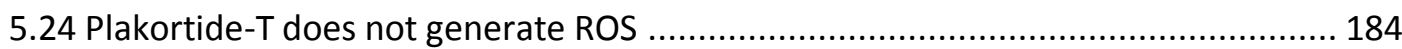

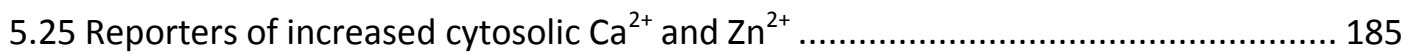

5.26 Plakortide-T causes an increase in cytosolic $\mathrm{Ca}^{2+}$ concentration which requires calcineurin

5.27 Effects of calcineurin activation in the $\Delta p d r 1 \Delta p d r 3$ background .......................... 189

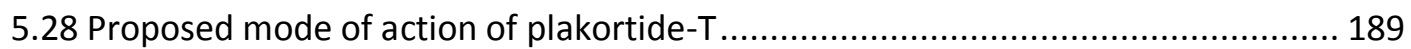

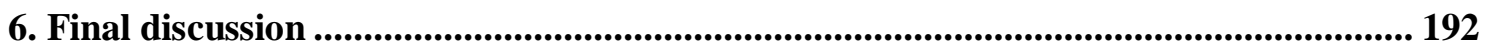

6.1 The utility of the PDR-deficient strains and PD-DMA ............................................ 192

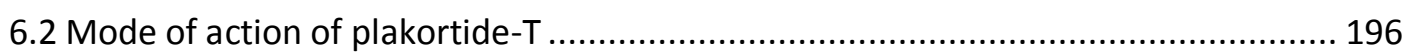

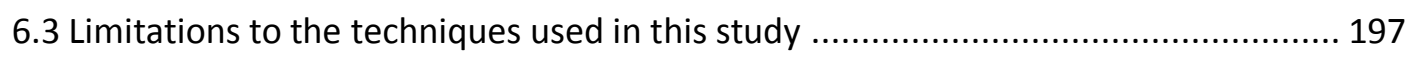

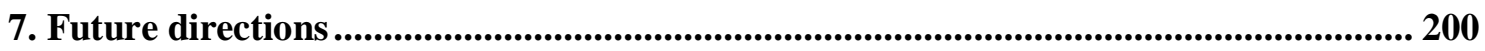

7.1 The importance of plakortide-T drug target elucidation and identification................... 200

7.2 Future studies for elucidating the mechanism of action of plakortide-T ...................... 201

7.2.1 Resistant mutant generation and identification of the resistance mutation(s)...... 201

7.2.2 Sensitivity of rho (0) mutants to plakortide-T ...................................................... 201

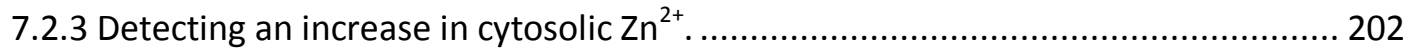

7.2.4 Unfolded protein response (UPR) induction assay............................................. 202

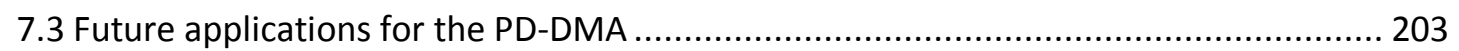

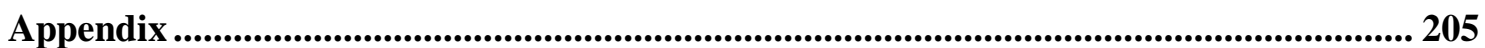

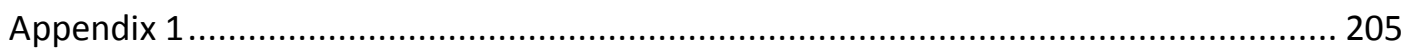

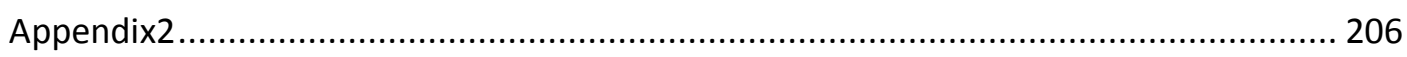

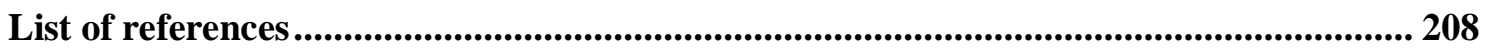




\section{Table of Figures}

Figure 1.1 - Chemical genetic interactions can mimic synthetic lethal interactions

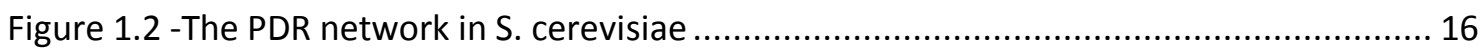

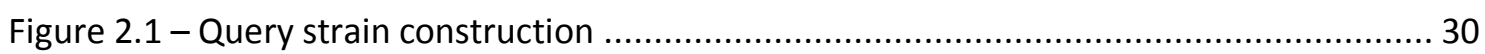

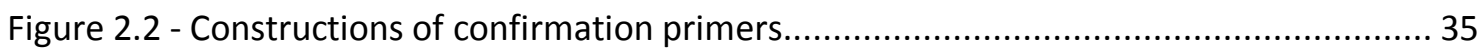

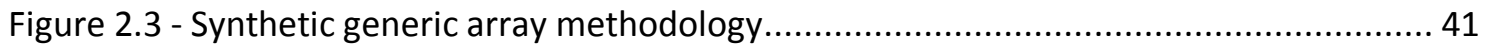

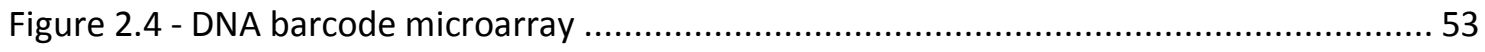

Figure 3.1 - Confirmation of P4339 and pAG60 plasmid isolation .......................................67

Figure 3.2 - Construction of the natR and URA3 deletion cassettes....................................69

Figure 3.3 - Confirmation of correct genomic insertion of $p d r 1 \Delta:: n a t R$ and $p d r 3 \Delta:: U R A 3 \ldots \ldots . .70$

Figure 3.4 - Differential sensitivity to the PDR-substrate cycloheximide............................... 71

Figure 3.5- PEs resulting from reduced mating efficiency of gene deletion mutants ............... 77

Figure 3.6- Confirmation of putative PEs with PDR-deficient strain..................................... 79

Figure 3.7- PEs resulting from reduced sporulation efficiency of gene deletion mutants......... 81

Figure 3.8 - Loss of Pdr1p and Pdr3p transcription factors does not induce nor impair

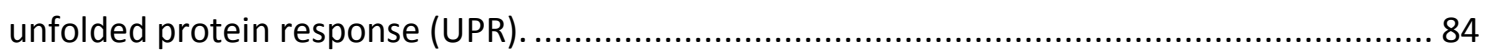

Figure 3.9 - Loss of Pdr1p and Pdr3p transcription factors does not induce or impair

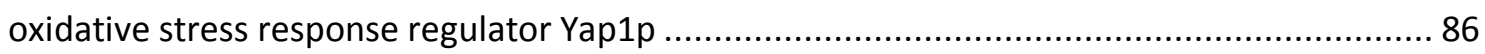

Figure 3.10- Differential sensitivity of the deletion mutants pools to PDR substrate

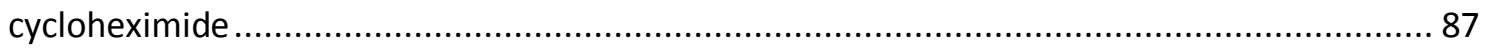

Figure 3.11 - Network diagram of genes hypersensitive to $5 \mathrm{nM}$ rapamycin ...........................90

Figure 3.12 - Network diagram of genes hypersensitive to $30 \mathrm{nM}$ cycloheximide

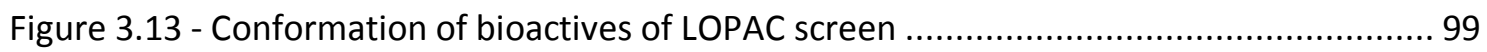

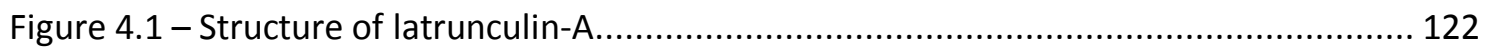


Figure 4.2- PD-DMA are more sensitive to latrunculin-A.

Figure 4.3 - Latrunculin-A is a Pdr5p substrate.

Figure 4.4 - Network diagram for genes hypersensitive to $28 \mathrm{nM}$ latrunculin-A

Figure 5.1 - Structure of plakortide-T

Figure 5.2- The PD-DMA strains are sensitive to Plakortide-T and not the DMA

Figure 5.3- The PDR-deficient strain are sensitive to Plakortide-T and not the wild-type....... 150

Figure 5.4- Plakortide-T cytotoxicity assay.....

Figure 5.5 - The major drug efflux transporters do not individually contribute to

plakortide-T resistance.

Figure $5.6-\mathrm{Ca}^{2+}$ and $\mathrm{Zn}^{2+}$ increases the potency of Plakortide-T in a synergistic manner. 154

Figure 5.7 - EGTA rescue of plakortide-T induced growth inhibition 155

Figure 5.8 - Sensitivities of calcium and transport mutants and calcineurin mutants to

$\mathrm{CaCl}_{2}, \mathrm{ZnCl}_{2}$ and plakortide-T. 158

Figure 5.9 - Plakortide-T does not induce the oxidative stress response regulator Yap1p..... 163

Figure 5.10 - Plakortide-T does not generate ROS detectable by the DTNB assay...... 164

Figure 5.11 - Crz1p-GFP as reporter for increase in intracellular calcium 166

Figure 5.12 - Reporter for increase in intracellular zinc 170

Figure 5.13 - Plakortide-T treatment causes an increase in cytosolic calcium . 171

Figure 5.14 - Location of Crz1p-GFP in calcium and zinc homeostasis mutants in response to treatment with plakortide- $\mathrm{T}, \mathrm{CaCl}_{2}$ and $\mathrm{ZnCl}_{2}$

Figure 8.1 - Structures of LOPAC library compounds that showed biological activity in yeast. 


\section{Table of Tables}

Table 2.1 - Yeast strains used in this chapter

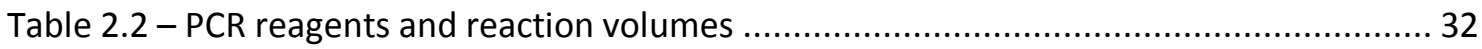

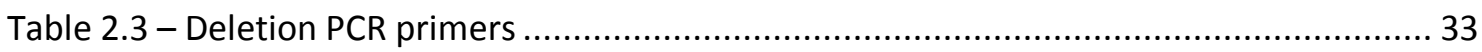

Table 2.4 - PCR reagents and volumes for construct confirmation PCR …............................. 34

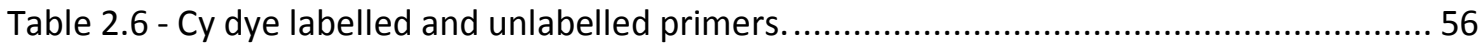

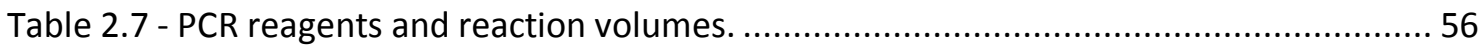

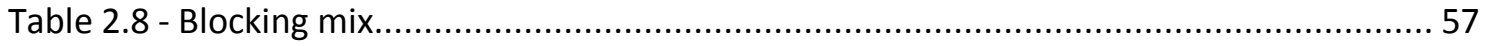

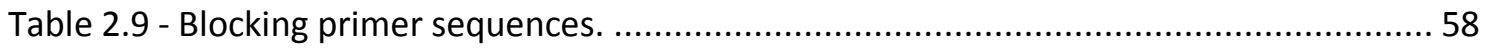

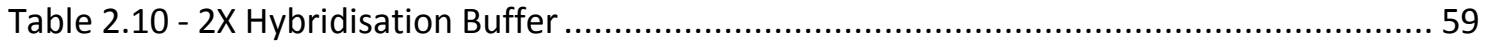

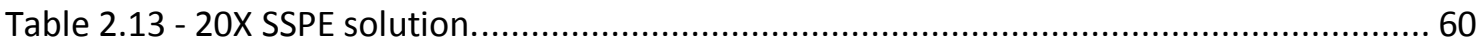

Table 3.1 - Putative phenotypic enhancements of the PDR-deficient strain ........................... 74

Table 3.2- Confirmation of putative PEs with PDR-deficient strain .................................... 80

Table 3.3 - PEs resulting from reduced sporulation efficiency of gene deletion mutants......... 82

Table 3.4 - List of gene deletion mutants hypersensitive to 5nM rapamycin .......................... 90

Table 3.5 - List of gene deletion mutants hypersensitive to $30 \mathrm{nM}$ cycloheximide

Table 3.6 - PDR-deficient mutants identifies more bioactives from the LOPAC library 98

Table 4.1- List of genes deletion mutants hypersensitive to $28 \mathrm{nM}$ latrunculin-A in a

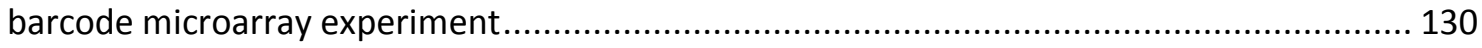

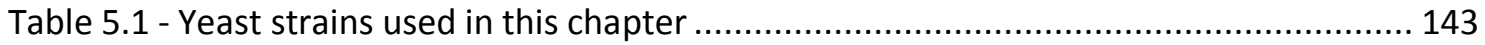

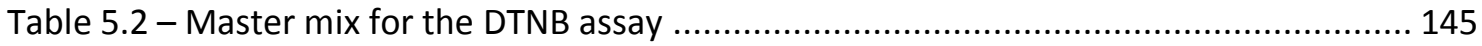

Table 5.3 - List of genes deletion mutants hypersensitive to $160 \mathrm{nM}$ plakortide-T in a barcode microarray experiment

Table 8.1 - The complete List of genes deletion mutants hypersensitive to $28 \mathrm{nM}$ latrunculin-A in a DNA barcode microarray experiment 


\section{$\underline{\text { List of Abbreviations }}$}

\begin{tabular}{|c|c|}
\hline $\mathrm{ABC}$ & ATP binding cassette \\
\hline BD & Becton, Dickinson \& Company. \\
\hline bp & base pairs \\
\hline ClonNAT & Nourseothricin \\
\hline DBD & DNA binding domain \\
\hline $\mathrm{dd} \mathrm{H}_{2} \mathrm{O}$ & Distilled deionised water \\
\hline DMA & Deletion Mutant Array \\
\hline DMSO & Dimethyl sulfoxide \\
\hline DTT & Dithiothreitol \\
\hline EDTA & Ethylenediaminetetraacetic Acid \\
\hline G418 & Geneticin \\
\hline GFP & greeen florecent protein \\
\hline GO & Gene Ontology \\
\hline HIP & haploinsufficiency profiling \\
\hline HOM & homozygous profiling \\
\hline KEGG & Kyoto Encyclopaedia of Genes and Genomes \\
\hline LB & Luria-Bertani Media \\
\hline LBD & ligand binding domain \\
\hline LOPAC & library of pharmacologically active compounds \\
\hline MDR & multi-drug resistance \\
\hline MFS & major facilitator superfamily \\
\hline MIC & minimum inhibitory concentration \\
\hline MSE & multi-drug efflux \\
\hline NBD & nucleotide binding domains \\
\hline nt & nucleotides \\
\hline OD & Optical Density \\
\hline ORF & Open Reading Frame \\
\hline PD-DMA & PDR-deficient DMA \\
\hline PDR & Pleiotropic drug resistance \\
\hline PDRE & PDR element \\
\hline PE & Phenotypic Enhancement \\
\hline PEG & Polyethylene glycol \\
\hline PFA & plakortide $\mathrm{F}$ acid \\
\hline
\end{tabular}




$\begin{array}{ll}\text { P-gp } & \text { P-glycoprotein } \\ \text { ROS } & \text { Reactive Oxygen Species } \\ \text { SAR } & \text { Structure Activity Relationship } \\ \text { SC } & \text { Synthetic Complete Media } \\ \text { SD } & \text { Synthetic Dropout media } \\ \text { SDS } & \text { Sodium dodecyl sulphate } \\ \text { SESA } & \text { SGA Experimental Set Analyser } \\ \text { SGA } & \text { Synthetic Genetic Array } \\ \text { SGAM } & \text { Synthetic Genetic Array Mapping } \\ \text { SGD } & \text { Saccharomyces Genome Database } \\ \text { SL } & \text { Synthetic Lethal } \\ \text { SPO } & \text { Enriched sporulation media } \\ \text { SS } & \text { Synthetic Sick } \\ \text { TBE } & \text { Tris/Borate/Edta } \\ \text { TCA } & \text { Trichloroacetic Acid } \\ \text { TE } & \text { Tris Hcl/Edta } \\ \text { TMD } & \text { trans-membrane domains } \\ \text { UPR } & \text { unfolded protein response } \\ \text { UPRE } & \text { UPR element } \\ \text { UV } & \text { Ultraviolet } \\ \text { wt } & \text { wild-type } \\ \text { xxx } & \text { Deletion from the DMA } \\ \text { YGDS } & \text { Yeast Genome Deletion Set } \\ \text { YPD } & \text { Yeast extract Peptone Dextrose media } \\ & \end{array}$




\section{Introduction}

\subsection{Natural products}

This study looks to expand on the current preliminary drug screening assays, and it specifically looks at natural products to identify biologically active, pharmacologically relevant compounds. Crude extracts derived from living organisms have been used to treat human ailments with the earliest recorded use of plants extending back as far as some 60,000 years ago (1). The pharmaceutical interest in these naturally occurring substances peaked between the years 1970-1980 and by 1990s, $80 \%$ of all the available drugs were natural products or natural product derivatives (2). These included antibiotics (i.e. Penicillin, tetracycline and erythromycin), anti-fungals (i.e. nikkomycins), anti-parasitics (i.e. avermactin), anti-malarials (i.e. quinine and artemisinin), cholesterol reducing agents (i.e. pyripyropene-A, lovastatin and derivatives), immunosuppressants (i.e. cyclosporine, FK506 and rapamycin) and antitumour agents (i.e. taxol, taxotere, dolastatin-10, echinomycin-A and doxorubicin) and central nervous system active agents (i.e. xenovulene-A) $(2,3)$. These compounds have revolutionised medicine extending the average human life expectancy of 44 in the early twentieth century to more than 77 years today (2).

Despite the past successes of natural products in medicine, pharmaceutical research into natural products has declined over the past two decades in favour of high throughput screening of synthetic libraries on defined molecular targets $(4,5)$. These efforts have been largely unsuccessful and have contributed to a decline in the discovery and development of novel-drug like molecules $(5,6)$. With multi-drug resistant and 
extensively resistant pathogens on the rise, there is a perpetual need for new antibiotics $(4,6)$. Most of the currently available antibiotic classes are expected to be no more effective in the future than they are today and the inevitable rise of the resistance mutants will erode away the effectiveness of these antibiotics in the future $(4,6)$. The recent decline in novel therapeutic discovery has greatly contributed to the rise of resistant isolates (6). Therefore, the need for a new generation of antibiotics is of importance.

Since 1960 only four new classes of antibiotics have been discovered. However, none have made a significant contribution to the pharmacopeia which is still dominated by antibiotic classes discovered more than a half a century ago (6). The antibiotics currently in use are generated through chemical tailoring of these few, and other wellworn scaffolds (6). Although making incremental changes to existing scaffolding is a good short term strategy for creating new antibiotics, a sustainable solution to combat resistance will require the discovery of novel scaffolds (6).

The synthetic high throughput screening libraries responsible for the recent decline in natural product research are typically created by making either incremental changes to the basic compound skeleton or tailoring of well characterised scaffolds, tinkering with substituents while leaving the core structure in tact (4). The results were initially promising as tailoring of scaffolds generated analogs with improved properties over parental compounds, for example overcoming drug resistance mechanisms (4). However, it was soon noted that starting with structurally similar compounds leads to compounds with similar biological activity that occupy only a small window in 
biological space (7). These synthetic libraries lack the structural diversity observed in natural product libraries. Furthermore, making incremental changes to well-worn scaffolds will only be effective in the short term due to existing resistance to several of these core structures (6).

In contrast, natural products are complex structures consisting of numerous oxygen containing substituents and an abundance of chiral centres with steric chemistry $(4,5)$. These complex structures have undergone evolutionary selection pressures to interact with various other biological targets in wide variety of organisms. Therefore, they can be considered as privileged structures (5). Natural products occupy a region of chemical space that compliments synthetic compounds and the hit rate for natural products are estimated to be $\sim 100$ fold greater than of synthetic libraries (4). Therefore, natural product research is an invaluable lead for yielding novel drug leads (5).

\subsection{Yeast as a model organism}

The search for novel therapeutic agents with novel mechanisms requires simple and inexpensive laboratory screens (8). One such screen involves using baker's yeast Saccharomyces cerevisiae as a model organism. The unicellular eukaryote $S$. cerevisiae has a small size and short life cycle with a population doubling time of 90 minutes under optimum conditions, and relative ease of culturing in media makes it ideal for laboratory use (9). 
S. cerevisiae was the first eukaryote to be transformed with a plasmid and the first eukaryote to be constructed with a precise gene deletion (8). It was also the first organism to have the entire genome sequenced and a map of its genome was released in 1996 (10). S. cerevisiae has a small genome consisting of 16 chromosomes, comprising 6000 open reading frames (ORFs) that are well characterised $(10,11)$. The yeast genome is genetically tractable, with easily manipulated genes that can be introduced in and out of the genome with relative ease $(9,12,13)$. Nearly 1,000 yeast genes have orthologs that are implicated in human disease and a majority of these have mammalian homologs that are functional in yeast and compliment yeast loss of function mutations (13). The conservation of amino acid sequence and gene function across taxa makes it possible to infer gene function identified in yeast to other eukaryotes (13).

S. cerevisiae has both haploid and diploid life cycles. Its ability to reproduce as haploids makes it easier to generate and maintain true breeding mutants $(8,9,14)$. Its diploid life cycle permits the introduction of gene mutations by mass mating as well as allowing genetic techniques such as complementation and epitasis (8).

Of the $\sim 6000$ ORFs in S. cerevisiae only $\sim 1000$ are indispensable for viability $(11,15)$. The remaining $~ 5000$ are non-essential genes and have been systematically replaced by integration of an antibiotic resistance marker and a synthetic barcode sequence to create a comprehensive non-essential deletion mutant array (DMA) (16). The antibiotic resistance marker kanR confers resistance to geneticin (G418) an analog of kanamycin (17). The synthetic barcode sequence comprise of two unique 20 nucleotide tags (UP tag and DN (down) tag) flanking the $k a n R$ cassette $(16,17)$. The UP and DN tags are 
unique to each gene deletion, allowing parallel analysis of the entire yeast genome-wide deletion set as a pool. The abundance of barcodes reflects the abundance of individual yeast strains under a given condition and allows quantitative analysis of the fitness of individual yeast strains grown as a pool (17). The barcodes denoting the deletion mutants are utilised in DNA barcode microarray technology to identify yeast gene deletion mutants that are sensitive or resistant to a given condition (15).

The integration of kanR deletion cassettes into the yeast genome has generated a comprehensive genome wide gene deletion set in both haploid and diploid organisms $(16,18)$. The haploid genome wide deletion set is utilised in Synthetic genetic array (SGA) methodology to generate double mutants by mass mating against a query gene deletion to identify genetic interaction networks (11). Other comprehensive mutant libraries have been constructed, including the green florescent protein (GFP) fusion library to visualise protein localisation which allows the study of genetic interactions which do not produce a defect in growth phenotype (13).

Genetic interaction data available for $S$. cerevisiae are unmatched by other models organisms (15). $\sim 85 \%$ of the protein coding genes in S. cerevisiae have been assigned with a biological function (13). Other resources on $S$. cerevisiae include the Saccharomyces Genome Database (SGD; http://www.yeastgenome.org/) which provides literature based information on gene function for every gene that has been studied (13). The lessons learned in yeast are transferable to other organisms due to gene conservation across taxa. 


\subsection{Various strategies for drug target elucidation and identification}

The identification of compound targets often remains the bottleneck in drug discovery programs (19). There are several strategies employed for elucidation of drug targets which can be categorised into either direct or indirect approaches. Affinity chromatography is the most utilised direct approach which involves immobilisation of a compound of interest on a solid surface (via a either biotin affinity tag, affi-gel tag or click chemistry) and treatment with proteins from a cellular lysate (20). The proteins bound to the compound are purified by gel separation and are analysed by tandem mass spectrometry (21). Affinity chromatography has been utilised in the identification of numerous compound targets including FK-506, trapoxin and chromeceptin (22).

Another direct approach is the use of yeast three-hybrid system. The yeast three-hybrid system utilises a DNA binding domain (DBD) attached to a ligand binding domain (LBD), a transcriptional activation domain (AD) bound to a second LBD and a bivalent hybrid ligand (23). The hybrid ligand comprises an 'anchor moiety' which attaches the hybrid ligand to the LBD of the $\mathrm{DBD}$, and the test compound. These two moieties are covalently linked (23). When the test compound interacts with the LBD of the AD, AD fusion protein is recruited to the DNA and leads to expression of a reporter gene. The utility of the yeast three-hybrid system in drug target identification was demonstrated by identification of FKBP12 at the target of FK-506 by Licitra et al. (24).

Another direct approach is mRNA display. The mRNA display utilises the mRNA transcribed from a dsDNA library and the mRNA is linked to a puromycin 
oligonucleotide (25). The mRNA-puromycin oligonucleotide is translated in vitro to produce a protein cross linked to its mRNA (25). The resulting mRNA-protein fusion molecules are then reverse transcribed to present cDNA (25). The compound of interest is immobilised on a solid surface and are incubated with mRNA-protein-cDNA fusion library (21). The unbound mRNA-protein-cDNA is washed off. The bound proteins are eluted, the cDNA is purified and sequenced and the target is identified by sequence similarity (21).

The direct studies mentioned above are limited by the loss of bioactivity as a result of attachment to affinity tags or solid matrices. As natural products are complex structures with numerous chiral centres, attachment to a solid resin may be difficult without compromising its biological activity. Therefore, structure activity relationship (SAR) studies must be performed to identify structures that are not necessary for biological activity, before attachment to the solid resin (20). As natural products are limited in supply there may not be enough compound for an SAR study and subsequent affinity chromatography assays.

An indirect approach for drug target identification involves proteomic profiling, which studies protein spots on two-dimensional gel electrophoresis (20). The change in isoelectric point or molecular weight of a protein following posttranslational modification leads to a mobility shift of the protein spot (20). Therefore, change in gene expression or protein modification by a compound can be studied using proteomic profiling and these can be compared against compounds with known targets to identify the targets of novel compounds (20). 
The most commonly used indirect method is transcriptional profiling where cDNA is utilised to identify expression profiles of cells under compound treatment, which is then compared against expression profiles of other known compounds (22). The compounds with similar expression profiles have a similar mode of action therefore, drug targets can be elucidated by comparing expression profiles (22). Using this approach, the target of the anaesthetic, dyclonine was identified as ERG2 (22). 


\subsection{Chemical-genetic interaction profiling}

Another commonly used approach employs genetics to elucidate the mechanisms of action of compounds. Chemical-genetics is defined as the study of biological systems using compounds (26). It is based on the principle that a primary effect of a compound is to bind a gene product in a specific manner and inhibit its function (Fig.1.1a) (26, 27). The primary effect of a compound can therefore be mimicked by the deletion of its corresponding gene target (Fig.1.1b) (27).

There are several approaches for chemical-genetics in yeast which differ based on yeast mutant libraries used. Multi-copy suppression profiling (MSP), uses the yeast overexpression library (22). MSP assays are based on the principle that increasing quantity of a compound's target leads to resistance to a compound (22). A compound screen against the collection of over expression mutants will identify drug targets and pathways affected by mutants that confer resistance (22). Using this method, the targets of methotrexate, fluconazole and rapamycin have been identified as DFR1, ERG11 and TOR 1, respectively (22).

An inverse of this approach the haploinsufficiency profiling (HIP) which utilises the heterozygous gene deletion set. Each of the heterozygous deletion mutants carries half of the gene dosage for particular genes including the compound's target. Therefore, mutants with reduced dosage for a compound's target will demonstrate hypersensitivity to that compound. HIP assays therefore identify the direct target of compounds as well as other components in the same pathway (22). 
Analogous to this technique is the homozygous profiling (HOP) which utilises the homozygous gene deletion set in place of the heterozygotes. HOP assays study the effects of a compound upon complete loss of a gene (22). The HOP assay therefore mimics double deletion mutants where the compound mimics the second site mutation and identifies genes and pathways required to buffer the loss of the compound's target. From the pathways that are affected the compound's target can be elucidated. In some instances, the HOP assay identifies drug targets. An elegant illustration of such was the target identification of the immunosuppressant rapamycin (28). An alternative to the HOP assay is the use of haploid deletion mutants for chemical genetic profiling.

Screening the DMA against sub-lethal concentrations of a compound can be used as a forward genetics approach to dissect the underlying mechanism of a compound by means of synthetic enhancement (Fig.1.1c) (27). Synthetic enhancement is a genetic phenomenon in which a second site mutation enhances the phenotype of an initial genetic lesion to produce a growth defect where individual genetic lesions do not demonstrate such a phenotype (11). Synthetic lethality is an extreme form of synthetic enhancement where the combination of two loss-of-function mutations leads to inviability, whereas synthetic sick interactions only show a reduction in viability. (11).

Similarly, genome wide approaches are applied to a gene deletion of a compound target. Mass mating the gene deletion against the haploid DMA and measuring the fitness of the double mutant meiotic progeny for synthetic lethal/sick interactions, synthetic genetic interaction profiles can be generated. Synthetic genetic interaction 
profiles for a gene deletion should resemble the chemical-genetic interaction profile of its inhibitory compound (Fig.1.1d) (27).

Consequently, genetic interaction networks for a compound or a gene deletion identifies buffering mechanisms that compensate for a loss function of a gene (17). Hence, the mode of action of compound can be inferred from these genetic interaction networks in a guilt-by-association manner to dissect the target of a compound. Comparing a chemical-genetic interaction profile for a compound of interest against a compendium of existing genetic interaction profiles can be used to infer the compound's true target, making it a powerful tool for drug discovery (27). As the compendium of genetic interaction networks and the drugable gene targets grow, it will become easier to associate compounds with their true targets (26). These reasons mentioned above make S. cerevisiae the ideal organism to utilise in chemical genomic studies. 
a

Drug target Gene X Phenotype

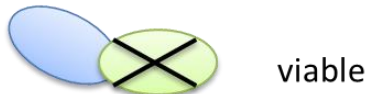

Drug

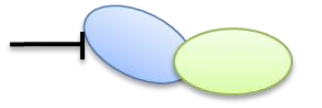

viable

Drug

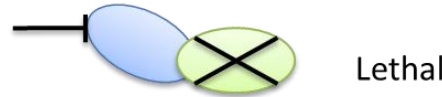

C

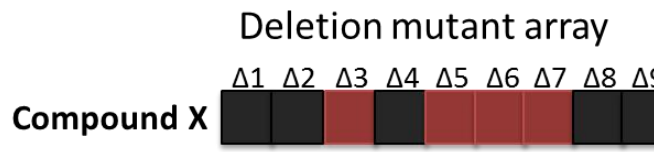

Chemical genetic interaction

d b
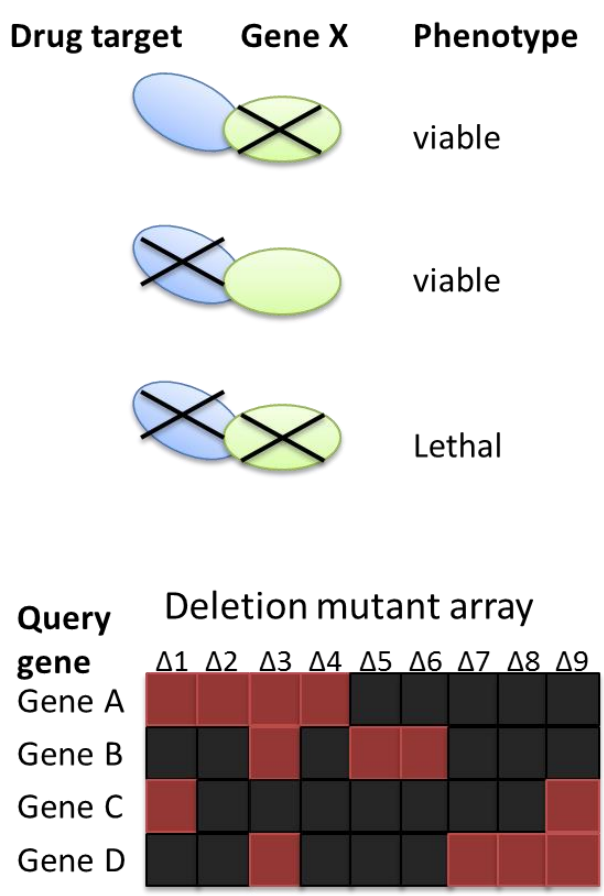

Synthetic-lethal interaction

Figure 1.1 - Chemical genetic interactions can mimic synthetic lethal interactions. (a) Chemical genetic interaction where a gene loss of function mutant shows a lethal/sick phenotype (growth defect) to otherwise sub-lethal concentrations of compound. Either alone is viable and has no growth defect but in combination shows lethal/sick phenotype. (b) A synthetic genetic interaction where two gene deletions in combination show epistatic interaction and a synthetic lethal/sick phenotype but individually, single gene deletions have no such defect.(c-d) A comparison of a chemical interaction profile against a compendium of genetic interaction profiles to identify the target of the compound of interest. In the diagram, a chemical genetic interaction profile of compound $\mathrm{X}$ resembles the genetic profile of query gene $B$, therefore, identifying the primary target of compound $X$ as gene $B$ (adapted from Boone et al. (17)). 


\subsection{Chemical-genetic interaction profiles in yeast}

Chemical-genetic interaction profiling for a compound can be performed either in liquid or solid media. The DMA comprising of $\sim 4700$ deletion mutants can be pinned onto agar plates containing a compound of interest at a semi-inhibitory concentration. The fitness of the deletion mutants is assessed by measuring colony size (27). Assessing the colony size of the DMA in the presence and absence of compounds for synthetic lethal or synthetic sick phenotypes are used to generate chemical-genetic interaction profiles specific for an inhibitory compound (27).

Alternatively, the DMA can be pooled and grown in liquid media under drug treated and untreated conditions. The fitness of the haploid mutant strains is assessed using microarray technology where the barcodes denoting the deletion mutants amplified with Cy3 (untreated) or Cy5 (compound treated) labelled primers and resulting PCR products are hybridised to a microarray chip containing oligonucleotides corresponding to each barcode (17). The competitive hybridisation of Cy3 and Cy5 labelled PCR products allows a quantitative readout of the fitness of each haploid mutant strain in drug treated and untreated conditions and are used to generate chemical-genetic interaction profiles (17).

Microarray based fitness analysis is more drug efficient than colony size-based fitness analysis as the entire DMA is analysed as a mixed population of mutants in two $10 \mathrm{~mL}$ cultures. In comparison, to assess the colony size by pinning the DMA on to solid media plates containing inhibitory compounds require each deletion mutants to be 
pinned as a single colony. This requires multiple agar plates requiring a minimum of $600 \mathrm{~mL}$ of media. Consequently, more compound is needed which may not be available, especially when using natural products. Microarray based chemical-genetic profiling therefore, has the unique advantage of dealing with small amounts of compounds in chemical-genetic profiling assays.

\subsection{Multi-drug resistance in yeast}

Although yeast is a good organism to model human metabolism it has several disadvantages. Mechanisms which enable the multi-drug resistance (MDR) phenotype among mammalian cells and pathogenic fungi are also conserved in S. cerevisiae (29). Yeast being a non-motile organism, have developed MDR or pleiotropic drug resistance (PDR) as means to escape unfavourable environments (29). The PDR phenotype is mediated by transcriptional upregulation of an armoury of plasma membrane transporters which pumps xenobiotics out of the cell or into cellular compartments for sequestering $(30,31)$.

ATP binding cassette $(\mathrm{ABC})$ proteins are the largest of six super-families which encode multi-drug efflux (MSE) systems $(31,32)$. ABC transporters are found in every cellular compartment with the exception of the ER and generally consist of four modules; 2 nucleotide biding domains (NBD) and 2 trans-membrane domains (TMD) arranged in $\left(\mathrm{TMD}_{6}-\mathrm{NBD}\right)_{2}$ forward or in reverse order $\left(\mathrm{NBD}-\mathrm{TMD}_{6}\right)_{2} \quad(30)$. NBDs are conserved across all $\mathrm{ABC}$ transporters and are responsible for ATP hydrolysis dependent transport 
of substrates (30). TMDs are variable and are presumed to be responsible for substrate recognition by $\mathrm{ABC}$ transporters $(30,33)$.

In $S$. cerevisiae there are $28 \mathrm{ABC}$ transporter genes. Nine of these encode for PDR transporters (32). These PDR transporters mediate the efflux of numerous structurally and functionally unrelated xenobiotics in an ATP-dependent manner leading to MDR $(30,31)$. In addition to the $\mathrm{ABC}$ transporters, proteins of the major facilitator superfamily (MFS) transporters also contribute to the PDR phenotype (34). At least 20 MFS proteins have been identified in S. cerevisiae with overlapping substrate specificity with ABC transporters (29).

\section{$1.7 P D R 1$ and $P D R 3$ are the master regulators of the PDR network}

The PDR phenotype is mediated mainly by the binuclear $\mathrm{Zn}(\mathrm{II})_{2}$ cys6 zinc finger regulators Pdr1p and its homolog Pdr3p and to a lesser degree by bZip family regulators Yrr1p and Yap1p (31). Master regulators Pdr1p and Pdr3p, in conjunction with other minor regulators, mediate the expression of PDR genes under both physiological and adverse conditions; forming the PDR network (Fig. 1.3) $(31,35)$. 


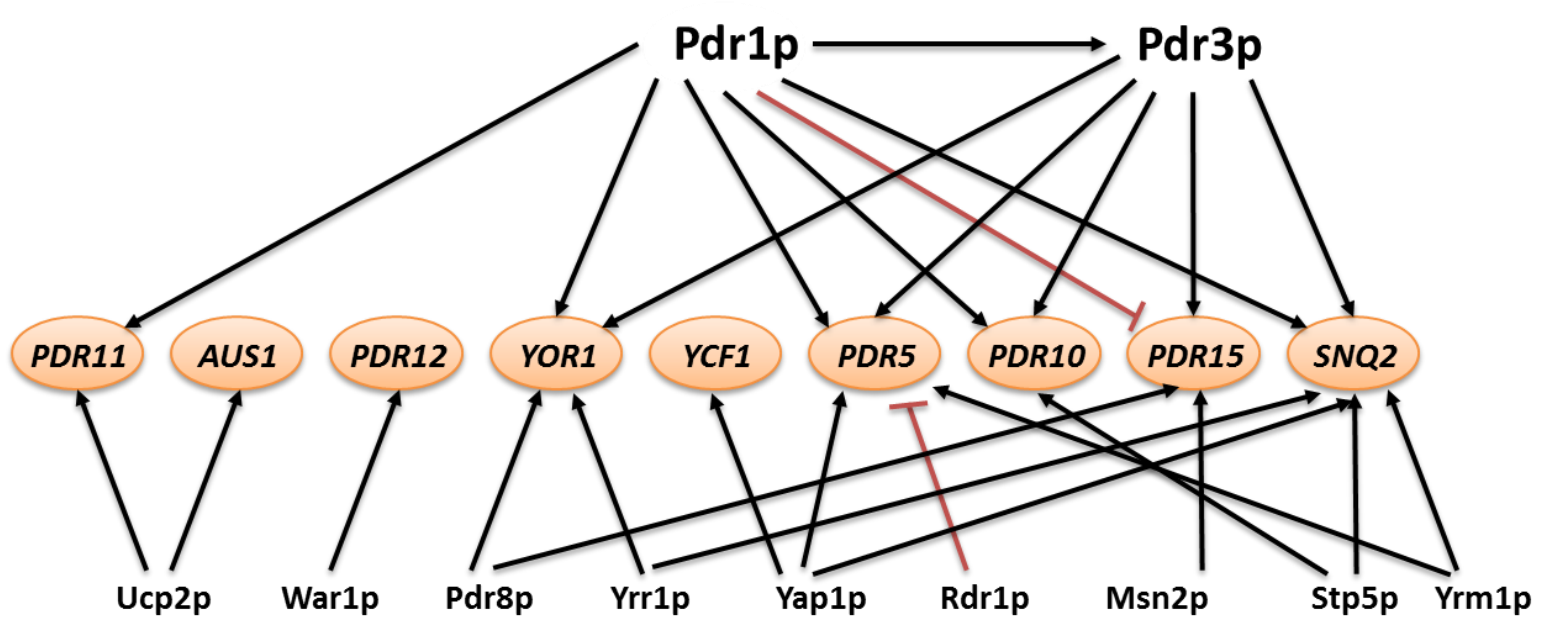

Figure 1.2 -The PDR network in S. cerevisiae. The target genes are represented in the centre and are out lined by circles. The transcriptional regulators are given above and below the target genes. The red arrows indicate negative regulation and the black arrows indicate positive regulation (reproduced from Jungwirth et al. (30)).

Under physiological conditions, the expressions of PDR network genes are predominantly under the control of Pdr1p and Pdr3p. The transcriptional regulation of PDR network genes by Pdr1p and Pdr3p is mediated by binding cis-acting elements present in promoters of target genes known as pleiotropic drug resistance elements (PDRE) (36). PDREs consist of the consensus sequence 5'-TCCGCGGA-3', a perfect palindrome (31). Through PDREs present within the promoter regions, Pdr3p and Yrr1p are auto-regulated $(31,35)$. Degenerate PDREs are also present within genes encoding for PDR pumps and serve as Pdr1p and Pdr3p targets $(35,36)$.

In addition, a target gene may have several PDRE consensus sequences within the promoter and the same consensus sequences within one promoter can have different induction patterns (37). The presence of multiple PDREs within target promoters 
enables regulation from different activators and inhibitors of the PDR network and is responsible for distinct regulation of target genes $(31,38)$.

Pdr1p and Pdr3p are phosphoproteins with Pdr3p having two distinct phosphorylation states. They can bind target promoters either as homo or hetero-dimers, or form heterodimers with other minor regulators of the PDR network such as Yrr1p, Stb5p and Rdr1p to 'fine-tune' the activity on target genes $(30,37,38)$. Through PDREs present in targets, Pdr1p and Pdr3p regulate the expression of the PDR network genes: PDR5, SNQ2, PDR15, PDR12, YOR1, PDR10 as well as various non-PDR genes $(31,39,40)$.

In response to stress, PDR network genes are regulated by Yap1p/Yrr1p and $\mathrm{C}_{2} \mathrm{H}_{2}$-zinc finger proteins Msn2p/Msn4p (35). Target recognition by Yap1p/Yrr1p and Msn2p/Msn4p is also achieved by recognising consensus sequences present on the target promoters (31). At least three Yap1p targets are directly controlled by Pdr1p and Pdr3p (40). The regulation of stress response by Pdr1p and Pdr3p suggests crosstalk between PDR response and stress-mediated response (35). An example of such is MFS protein Flr1p, a Yap1p/Yrr1p target which confers resistance to the agricultural fungicide benomyl in a Pdr3p dependent manner (41). The transcription factors Yap1p, Yrr1p, Msn2p and Msn4p mediate the expression of PDR network genes SNQ2, PDR5, YOR1, YCF1, PDR10, PDR12 and PDR15 under stress conditions. 


\subsection{Differential regulation of PDR network by Pdr1p and Pdr3p}

Pdr1p and Pdr3p, although paralogs, they are differentially expressed and may have opposing effects on targets $(29,31)$. Pdr1p is expressed at 10 times the level of Pdr3p and is responsible for drug induced expression of ABC and MFS transporters $(29,37)$. The loss of PDRl has a more pronounced effect on sensitivity to xenobiotics than the loss $P D R 3$, although, resistance to some compounds are more dependent on Pdr3p (34). PDR3 expression, however, is highly induced by mitochondrial defects, but not PDRl (37). Through PDREs present within the promoter region of PDR3, its expression is both auto-regulated and also positively regulated by $\operatorname{Pdr} 1 \mathrm{p}$ (30).

The expression of PDR network genes, specifically PDR5, PDR10 and PDR12 are differentially altered by deletions of $\Delta p d r 1$ and $\Delta p d r 3$ gene deletion mutants (36). Loss of PDRI led to a decrease in PDR5 expression, with no detectable effect on PDR10 expression and an increase in PDR15 expression (36). Conversely, the deletion of PDR3 has no effect on PDR5 expression, but decreased PDR10 expression and abrogated PDR15 expression (36). In another study deletion of PDRI showed complete down regulation of $P D R 5$ expression and deletion of $P D R 3$ leads to induction of $P D R 5$ expression (37). This suggests that Pdr3p may be a negative regulator of PDR5 expression while Pdr1p is a positive regulator. Pdr1p and Pdr3p therefore play nonidentical roles in mediating the PDR network and the PDR phenotype. 


\subsection{Chemical basis of substrate recognition by PDR pumps}

Although various PDR pumps overlap in their substrate specificity, the mechanism by which substrate specificity is achieved is yet unresolved. Several key features involved in substrate recognition by mammalian MDR transporter, P-glycoprotein (P-gp) have been identified: particularly substrate charge, hydrophobicity and the ability to form hydrogen bonds have been identified as crucial for substrate recognition, with water solubility of the substrate also playing a possible role $(42,43)$. These factors however are not important in substrate recognition by Pdr5p (the fungal equivalent of mammalian P-gp) the major drug efflux transporter in S. cerevisiae (44). The basis of substrate recognition by Pdr5p has been extensively studied with various structurally diverse compounds including tri-alkyltin chlorides, aromatic imidazoles, tetra-alkyltins, cycloheximide, chloramphenicol and rhodamine 6G. The surface volume of the compound was identified as the major determinant for substrate recognition (45-47). A surface volume $90 \AA^{3}$ or greater is required for Pdr5p substrates selection with optimum substrate recognition at $200-225 \AA^{3}(46)$. The substrates' ability to form hydrogen bonds also plays an important role in substrate selection by Pdr5p (43).

However, Pdr5p also recognises substrates which are significantly larger than the optimum surface volume determined for Pdr5p substrate selection. An example of such is Rhodamine 6-G, which has a surface volume of $480 \AA^{3}$ (46). It has been proposed that rhodamine 6-G consists of two set of chemical partners capable of interacting independently with Pdr5p and the distance between the two binding chemical moieties are relevant to substrate recognition (46). The PDR substrates with larger surface area may consist of multiple interacting chemical moieties where each chemical moiety may 
interact independently with Pdr5p to provide multiple binding options (46). Furthermore, at least 3 substrate binding sites have been identified for Pdr5p where different substrates bind several binding sites with differing affinities $(46,48)$.

\subsection{Hypersensitive deletion strain.}

The PDR network in S. cerevisiae prevents the compounds that serve as substrates for the PDR transporters from accumulating within the cells. Some substrates are so efficiently effluxed that they never accumulate to a cytosolic concentration sufficient for a growth inhibitory effect. This system must therefore be circumvented to determine the biological activity of these compounds that are PDR pump substrates. Currently, these compounds either have reduced potency in chemical screens in S. cerevisiae or do not demonstrate any bioactivity. Therefore these compounds will be ignored in chemical screens along with compounds that lack activity in yeast.

Currently, PDR pump mediated drug efflux is overcome by using PDR-deficient strains which are missing either one or more of the drug efflux transporters (49). PDRdeficient strains are constructed by either deleting the genes encoding for the pumps or deleting the genes encoding for the transcriptional activators that induces the expression of PDR pumps (49). A study by Rogers et al. included a PDR-deficient strain lacking in both PDRI and PDR3 as well as deletions in seven of the PDR transporters (47). Although PDR-deficient strains do exist, they have not yet been incorporated into the genetic background of the DMA to sensitise the entire deletion set. 
The master regulators, Pdr1p and Pdr3p play non-identical roles in modulating the PDR network leading to differential expression of PDR network genes. $(31,36)$. Therefore, we hypothesize that null mutations in both master regulators $P D R I$ and $P D R 3$ are required for an effective knockdown of the PDR network and would render $S$. cerevisiae hyper-sensitive to various unrelated xenobiotics. However, this is not a complete knockdown since other transcriptional regulators such as Yap1p and Yrr1p are still functional and $\mathrm{ABC}$ transporters and the MFS transporters they regulate in response to stress have overlapping specificity for substrates that are targeted for efflux by Pdr1p and Pdr3p targets (29). Ideally, these lesser regulators of the PDR network should also be knocked-out to attain a query strain and consequently a DMA deficient in PDR phenotype, but as a result of limited selectable markers, construction and selection, a query strain of such scale was not feasible.

Alternatively, yeast membranes can be permeabilised to compounds by using mutants of the ergosterol biosynthesis pathway such as erg6 or adding the detergent sodium dodecyl sulphate (SDS) (50). However, these can have undesirable effects on yeast. Loss of ERG6 increases the membrane permeability to sodium and lithium ions as well as reducing transformation efficiencies and sexual conjugation (50). Addition of SDS causes reduction in cell viability and membrane disruption permits the entry of various toxins as well as leakage of cellular content (50). 


\subsection{Hypersensitive DMA}

In this thesis I propose constructing the query strain with gene deletions in PDRI and $P D R 3$ in the wild type background and mating it to the genome wide deletion set to create a DMA with the background of null mutations in PDRland PDR3 transcription factors. Such a DMA would be hypersensitive to compounds that are normally targeted for efflux by PDR network pumps. PDRI and PDR3 are non-essential genes (38), therefore, only a few gene deletion mutants should show synthetically lethal/sick interactions with $\Delta p d r l$ and $\Delta p d r 3$ mutations and the resulting triple mutant DMA should be representative of the non-essential deletion mutant array.

To create the hyper sensitive DMA, PDRland PDR3 ORFs will be replaced by PCR mediated gene disruption with selectable markers in the wild type MAT $\alpha$ starting strain Y7092 and mated against the ordered array of haploid non-essential deletion mutants in a synthetic genetic array (SGA) $(17,51)$. In a series of replica pinning procedures MATa meiotic progeny carrying triple mutants will be selected (51). This will result in an ordered array of deletion mutants with the $\Delta p d r 1 \Delta p d r 3$ background which are hyper sensitive compounds of interest (52).

These hyper sensitive triple mutants will then be used in the place of the haploid wildtype background DMA for barcode microarray based fitness analysis to construct chemical-genetic profiles of novel compounds. The DMA in the $\Delta p d r 1 \quad \Delta p d r 3$ background can also serve as the MATa deletion mutant array in SGA analysis. Each of the deletion mutants can serve as starting strains to introduce additional mutations while 
maintaining the drug hypersensitive phenotype. The PDR-deficient DMA (PD-DMA) will serve as a powerful tool in dissecting the mechanism of novel natural products that serve as substrates for PDR network pumps. Natural products that may not possess pharmacokinetic properties useful for therapeutic purposes may still be utilised as probes to study novel biology in chemical genetic studies (7)

The chemical-genetic screens in the current DMA for PDR substrates requires flooding yeast cells with high concentrations of compounds to overcome the PDR mediated efflux, thereby triggering other 'damage control' (general stress) mechanisms. Example of such the induction Msn2p and Msn4p targets in response to treatment with xenobiotics 2,4-dichlorophenol and polyoxyethylene-9-laurylether (53). The induction these genes were significantly reduced in the $\Delta p d r 1 \Delta p d r 3$ double deletion background (53). The PDR-deficient system therefore, avoids such off target effects by inducing cell inhibition at lower concentrations without flooding the cellular machinery, thereby reducing the effective minimum inhibitory concentration (MIC) as well as off target toxicity. 


\section{Materials and Methods}

\subsubsection{Yeast strains}

All $S$. cerevisiae strains used in this chapter were derived from the diploid strain BY4743 (54) and were constructed by PCR mediated disruption (see query strain construction) and are as follows:

\begin{tabular}{|c|c|}
\hline Strain & Genotype \\
\hline BY4743 & 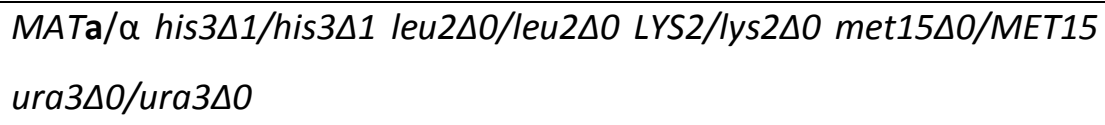 \\
\hline Y7092 (wild-type) & 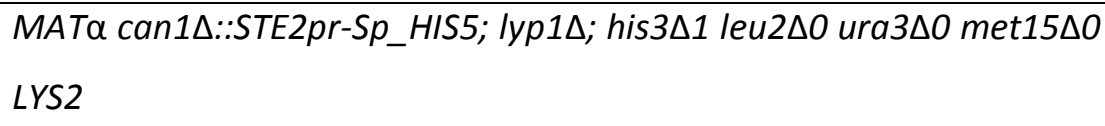 \\
\hline$\Delta p d r 1$ & 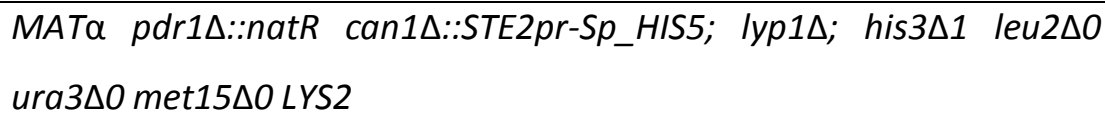 \\
\hline $\begin{array}{l}\Delta p d r 1 \Delta p d r 3 \\
\text { (Y7092 derived) }\end{array}$ & 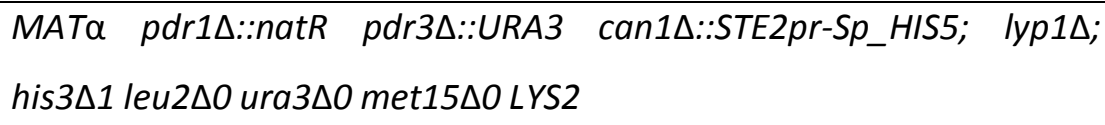 \\
\hline$\Delta u r a 3$ & 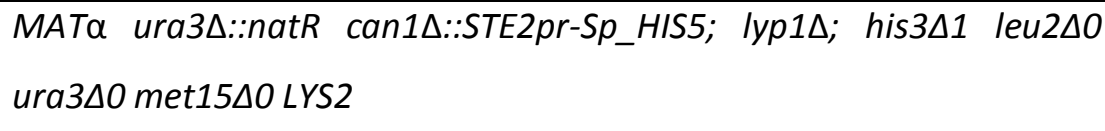 \\
\hline$\Delta x x x$ & MATa $x x x \Delta:: k a n R ;$ his $3 \Delta 1$ leu $2 \Delta 0$ ura $3 \Delta 0$ met $15 \Delta 0$ \\
\hline Y8205 & 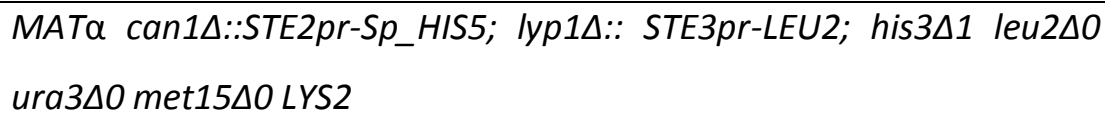 \\
\hline YAP1-GFP & MATa YAP1-GFP-HIS5 his $3 \Delta 1$ leu2 $\Delta 0$ ura $3 \Delta 0$ met15 $\Delta O L Y S 2+$ \\
\hline $\begin{array}{l}\text { YAP1-GFP } \triangle p d r 1 \Delta p d r 3 \\
\text { (Y8205 derived) }\end{array}$ & 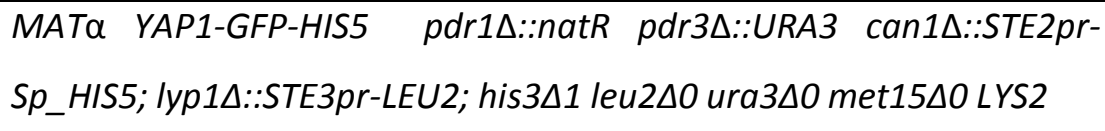 \\
\hline $4 \times U P R E-G F P$ & 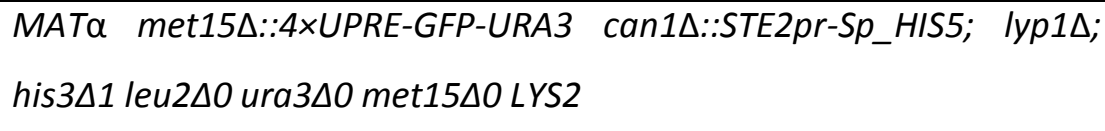 \\
\hline $\begin{array}{l}4 \times U P R E-G F P \triangle p d r 1 \triangle p d r 3 \\
(4 \times U P R E-G F P \text { derived) }\end{array}$ & 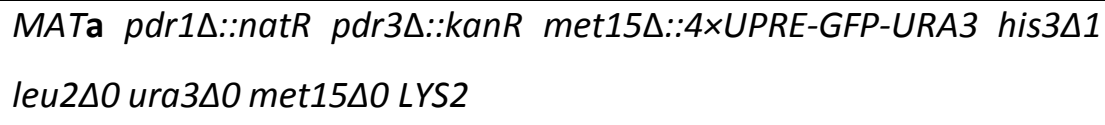 \\
\hline
\end{tabular}

Table 2.1 - Yeast strains used in this chapter. 


\subsubsection{Growth media and components}

All $S$. cerevisiae strains were cultured in one of the following growth media supplemented with the appropriate antibiotics; ClonNAT (Werner bioAgents) final concentrations of $100 \mu \mathrm{g} / \mathrm{mL}$ and geneticin (G418) final concentrations of $200 \mu \mathrm{g} / \mathrm{mL}$. The toxic amino acid analogs canavanine (Sigma-Aldrich) and thialysine (SigmaAldrich) were used at a final concentration of $50 \mu \mathrm{g} / \mathrm{mL}$ in the absence of their amino acid analogs. All media was autoclaved at $121^{\circ} \mathrm{C}$ for 22 min and cool to $65^{\circ} \mathrm{C}$, before adding $2 \%$ glucose and antibiotics unless otherwise stated.

Yeast peptone dextrose (YPD) agar: 1\% (w/v) yeast extract (Becton, Dickinson and Company), $2 \%$ (w/v) bacto-peptone (Becton, Dickinson and Company), 0.12\% (w/v) adenine (Sigma-Aldrich), 2\% (w/v) g agar (Invitrogen) and 2\% (w/v) glucose (SigmaAldrich). YPD broth was prepared as above without addition of agar.

Synthetic complete (SC) agar $0.17 \%(w / v)$ yeast nitrogen base without amino acids or ammonium sulphate (Becton, Dickinson and Company), 0.1\% (w/v) monosodium glutamate (Sigma-Aldrich), 0.2\% (w/v) amino acid mixture to suit (Sigma-Aldrich), 2\% (w/v) agar (Becton, Dickinson and Company) and 2\% (w/v) glucose (Sigma-Aldrich). SC broth was prepared as above without addition of agar.

Synthetic dropout (SD) agar: As with SC minus the appropriate amino acid supplement. SD broth was made without the addition of agar. 
Enriched sporulation (SPO) agar: 1\% (w/v) potassium acetate (Scharlau Chemie S.A.), $0.1 \%(w / v)$ yeast extract (Becton, Dickinson and Company), 0.05\% (w/v) glucose (Sigma-Aldrich), 0.2\% (w/v) amino acid mixture to suit (Sigma-Aldrich), 2\% (w/v) agar (Becton, Dickinson and Company). Enriched sporulation broth was prepared as above without addition of agar.

Amino acids mixture for SC media are as follows: $3 \mathrm{~g}$ adenine, $2 \mathrm{~g}$ uracil, $2 \mathrm{~g}$ inositol, $0.2 \mathrm{~g}$ para-aminobenzoic acid, $2 \mathrm{~g}$ alanine, $2 \mathrm{~g}$ asparagines, $2 \mathrm{~g}$ aspartic acid, 2 $\mathrm{g}$ cysteine, $2 \mathrm{~g}$ glutamic acid, $2 \mathrm{~g}$ glutamine, $2 \mathrm{~g}$ glycine, $2 \mathrm{~g}$ histidine, $2 \mathrm{~g}$ isoleucine, $10 \mathrm{~g}$ leucine, $2 \mathrm{~g}$ lysine, $2 \mathrm{~g}$ methionine, $2 \mathrm{~g}$ phenylalanine, $2 \mathrm{~g}$ proleine, $2 \mathrm{~g}$ serine, $2 \mathrm{~g}$ threonine, $2 \mathrm{~g}$ tryptophan, $2 \mathrm{~g}$ tyrosine and $2 \mathrm{~g}$ valine.

Synthetic dropout (SD) amino acid mixture is a mixture of amino acids (Invitrogen) above minus the appropriate amino acid supplement. Amino acid mixture for enriched sporulation composes of $2 \mathrm{~g}$ histidine, $10 \mathrm{~g}$ leucine, $2 \mathrm{~g}$ lysine and $2 \mathrm{~g}$ uracil.

All E. coli strains were cultured in lysogeny broth supplemented with the antibiotic ampicillin (Sigma-Aldrich) final concentration of $100 \mu \mathrm{g} / \mathrm{mL}$.

Lysogeny broth (LB) Agar: 1\% (w/v) bacto-tryptone (Becton, Dickinson and Company), $0.5 \%$ (w/v) yeast extract (Becton, Dickinson and Company), $1 \%$ (w/v) $\mathrm{NaCl}$ (Scharlau Chemie S.A.) and adjusted to $\mathrm{pH} 7.5$ with $10 \mathrm{M} \mathrm{NaOH}$. The media was 
autoclaved at $121^{\circ} \mathrm{C}$ for $22 \mathrm{~min}$ and cool to $65^{\circ} \mathrm{C}$, before adding $2 \%$ glucose and antibiotics unless otherwise stated. LB broth is prepared as above without addition of agar.

\subsubsection{Compounds and chemicals}

Compounds: cycloheximide (Sigma-Aldrich), rapamycin, camptothecin (SigmaAldrich) ketoconazole (Sigma-Aldrich) and Latrunculin A (kindly provided by Peter Northcote, School of Chemical and Physical Sciences, Victoria University of Wellington) were dissolved in DMSO at a working concentration of $10 \mathrm{mM}$ and stored at $-20^{\circ} \mathrm{C}$.

Dithiothreitol (DTT) (Sigma-Aldrich) and tunicamycin (Sigma-Aldrich) was dissolved in $\mathrm{ddH}_{2} \mathrm{O}$ at the working concentration of $1 \mathrm{mM}$ and was stored at $-20^{\circ} \mathrm{C}$. Hydrogen peroxide $\left(\mathrm{H}_{2} \mathrm{O}_{2}\right)$ (Sigma-Aldrich) was dissolved in $\mathrm{ddH}_{2} \mathrm{O}$ at a working concentration of $10 \mathrm{mM}$ was stored at $4{ }^{\circ} \mathrm{C}$. Menadione was dissolved in ethanol at the working concentration of $100 \mathrm{mM}$ and was stored at room temperature.

\subsubsection{Query strain construction}

This project requires generation of "query strains" i.e. particular gene deletions selectable with nourseothricin (clonNAT) and uracil dropout synthetic media (SD-Ura), for performing synthetic genetic array (SGA) manipulations in order to derive a deletion mutant array (DMA) on a background of null mutations in PDRI and PDR3 transcription factors. 
The yeast strain Y7092 (55) which carries S288c background was the starting strain for query strain construction. The Y7092 strain carries gene deletions $\Delta$ his $3, \Delta l e u 2$, sura3 and $\Delta m e t 15$, therefore permitting the use of its wild-type genes as selection markers to introduce and maintain genetic manipulations (55). Y7092 carries STE2pr-Sp_HIS5, the Schizosaccharomyces pombi HIS5 under the control of the STE2 promoter. The $S p \_H I S 5$ is able to complement the his $3 \Delta l$ deletion mutation in the Y7092 background and confers histidine prototrophy in MATa cells expressing STE2 (55). The Y7092 strain also carries gene deletion mutations in the CANland LYPI loci which in wildtype yeast encode the plasma membrane permeases of arginine and lysine respectively (55). These selection markers are utilised in SGA methodology to select for the appropriate meiotic progeny.

With the purpose of creating a PD-DMA, the query strain $\Delta p d r 1 \Delta p d r 3$ was constructed by sequentially deleting the ORFs; YGL013C (PDR1) and YBL005W (PDR3), from the starting strain Y7092 (51). Firstly, the PDR1 locus of Y7092 was replaced with a $n a t R$ deletion cassette by homologous recombination to create a $p d r 1 \Delta:: n a t R$ strain (see Fig. 2.1). Secondly, the $P D R 3$ locus of the resultant transformant was replaced with a URA3 deletion cassette by homologous recombination to create a pdrl $\Delta::$ natR $p d r 3 \Delta:: U R A 3$ strain. As mentioned in Chapter 1, $\Delta p d r 1$ and $\Delta p d r 3$ mutations render yeast more sensitive to compounds that are substrates of the PDR pumps including xenobiotics and marine natural products (47). 
The natR deletion cassette was constructed by amplifying the natMX4 gene from the plasmid p4339 with deletion primers. Each 68 base pair deletion primer consists of an $18 \mathrm{bp}$ region with homology to the 5 ' or $3^{\prime}$ ends of the natMX4 cassette and the remaining 50 bp region with homology to the 5' or 3' flanking genomic regions of the PDRI locus. The natR gene confers resistance to the antibiotic clonNAT in yeast by monoacetylation of $\beta$-amino groups of the $\beta$-lysyl moiety of clonNAT rendering it inactive (56). The natR gene permits the selection of pdrl $1:: n a t R$ mutants in the presence of the antibiotic clonNAT.

In the second step, the $P D R 3$ locus of the resulting $p d r 1 \Delta:: n a t R$ strain was replaced with another deletion cassette, CaURA3MX4 by homologous recombination to generate

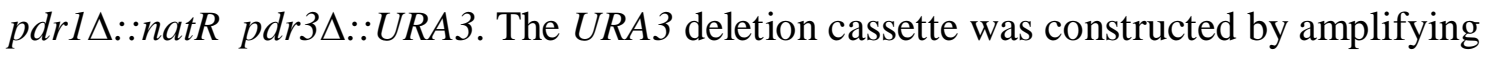
CaURA3MX4 gene from the pAG60 plasmid with the appropriate deletion primers. These deletion primers consist of $68 \mathrm{bp}$ : an $18 \mathrm{bp}$ region with homology to 5' or 3' ends of the CaURA3MX4 deletion cassette and the remaining 50 bp region with homology to the 5' or 3' flanking regions of the PDR3 ORF. The CaURA3MX4 carries URA3 gene of Candida albicans and confers uracil prototrophy in the transformed yeast strain. The $U R A 3$ gene in $p d r 3 \Delta:: U R A 3$ permits the selection of $\Delta p d r 3$ mutants in SD-Ura media. 
a
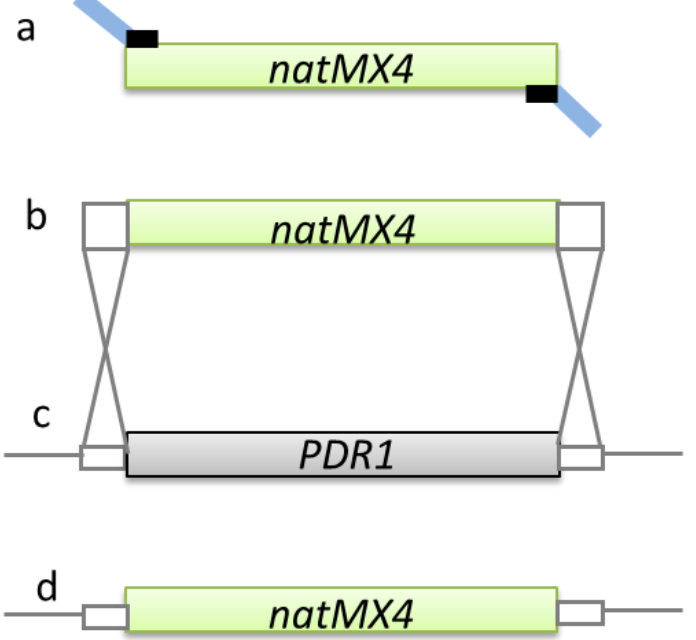

natMX4 cassette and deletion

primers

natR gene deletion cassette

PDR1 ORF

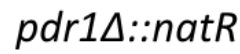

e

\section{natMX4}

Figure 2.1 - Query strain construction (adapted from Tong et al. (51)). (a) Blue bar designates the $50 \mathrm{bp}$ sequence of the deletion primer homologous to the $5^{\prime}$ or $3^{\prime}$ flanking region of the PDR1 locus. Black bar designates the $18 \mathrm{bp}$ sequence of the deletion primer homologous to the natMX4 cassette. (b) natR deletion cassette from the PCR reaction using deletion primers and natMX4 cassette. (b-c) schematic diagram of recombination event between natR gene deletion cassette and PDR1 locus leading to (d) $p d r 1 \Delta::$ natR deletion mutant. (e)The $\Delta p d r 3$ deletion is constructed in a similar manner where CaURA3MX4 uracil prototrophy cassette was used in place natMX4; deletion primers with homology to PDR3 flanking region and CaURA3MX4 cassette. Using $p d r 1 \Delta::$ natR as starting strain leads to $p d r 1 \Delta:: n a t R$ pdr3A::URA3 double mutant.

\subsubsection{Sub-cloning the plasmid vector}

DH5 $\alpha$ competent E. coli cells (Cat. No. 18265-017, Sub cloning Efficiency ${ }^{\mathrm{TM}}$ DH5 $\alpha^{\mathrm{TM}}$ Competent Cells, Invitrogen) were transformed with either pAG60 (Euroscarf) or p4339 (kind gift from Charles Boone, University of Toronto) plasmids according to manufactures guidelines. The AmpR gene carried by pAG60 and p4339 plasmids 
confers bacterial resistance to ampicillin (AMP) and permits the selection of bacteria transformed with plasmid.

\subsubsection{Plasmid mini-prep}

The pAG60 and p4339 plasmids maintained in DH5 $\alpha$ cells were purified using Zyppy $^{\mathrm{TM}}$ plasmid mini prep kit (cat. D4019, Zymo Research Cooperation) and the procedure was performed according to manufacturer's protocol. $5 \mu \mathrm{L}$ of purified plasmid DNA was electrophoresed on a $1 \%$ agarose gel stained with ethidium bromide and were visualised under UV (ultraviolet) light to confirm pAG60 and p4339 plasmid isolation.

\subsubsection{PCR amplification}

The Platinum ${ }^{\circledR}$ taq PCR amplification kit (Invitrogen, cat. 10966-034) was optimised with Q buffer (Qiagen) to amplify the natMX4 and URA3MX4 genes from the plasmids p4339 and pAG60, respectively to generate natR and URA3 deletion cassettes using deletion primers listed in Table 2.2.

The PCR reagents and volumes are given in Table 2.1 and all PCRs were performed using Techne TC-PLUS - Multiformat Thermal cycler (Bibby Scientific Limited, Staffordshire, UK). The reaction conditions were obtained from Janke et al. and were modified as follows: $94^{\circ} \mathrm{C}$ for 2 min for template denaturation and platinum Taq DNA polymerase activation, then 35 cycles of $95^{\circ} \mathrm{C}$ at $45 \mathrm{~s}$ for template denaturation, 52- 
$63.5^{\circ} \mathrm{C}$ temperature gradient for $40 \mathrm{~s}$ for primer annealing and $68^{\circ} \mathrm{C}$ for $1.5 \mathrm{~min}$ for primer extension, and finally, $68^{\circ} \mathrm{C}$ for $10 \mathrm{~min}$ to complete any partial primer elongations. $5 \mu \mathrm{L}$ of PCR products were electrophoresed on an $1 \%$ agarose gel stained with ethidium bromide and were visualised under UV to ensure the PCR product was the correct size.

\begin{tabular}{|l|l|}
\hline Reagents & Reaction volume $(\boldsymbol{\mu L})$ \\
\hline H2O & 14.15 \\
\hline Q buffer(Qiagen) & 5 \\
\hline 10x PCR buffer & 2.5 \\
\hline dNTP (5 mM) & 1 \\
\hline MgCl2 (50 mM) & 0.75 \\
\hline Forward deletion primer(100 mM) & 0.25 \\
\hline Reverse deletion primer(100 mM) & 0.25 \\
\hline Platinum taq & 0.1 \\
\hline Template & 1 \\
\hline Total & 25 \\
\hline
\end{tabular}

Table 2.2 - PCR reagents and reaction volumes.

\begin{tabular}{|l|l|}
\hline Primer & Sequence $\mathbf{5}^{\prime}$ to $\mathbf{3}^{\prime}$ \\
\hline PDR1 deletion primer forward & $\begin{array}{l}\text { CATCTCAGCCAAGAATATACAGAAAAGAATCCAAG } \\
\text { AAACTGGAAGACATGGAGGCCCAGAATACCCT }\end{array}$ \\
\hline PDR1 deletion primer reverse & $\begin{array}{l}\text { AGGAAGGAAGTTTTTGAGAACTTTTATCTATACAA } \\
\text { ACGTATACGTCAGTATAGCGACCAGCATTCAC }\end{array}$ \\
\hline PDR3 deletion primer forward & $\begin{array}{l}\text { ACTGCATCAGCAGTTTTATTAATTTTTTCTTATTGCG } \\
\text { TGACCGCAACATGGAGGCCCAGAATACCCT }\end{array}$ \\
\hline
\end{tabular}




\begin{tabular}{|c|c|}
\hline$P D R 3$ deletion primer reverse & $\begin{array}{l}\text { CCATTTACTATGGTTATGCTCTGCTTCCCTATTTCTT } \\
\text { TTGCGTTTCAGTATAGCGACCAGCATTCAC }\end{array}$ \\
\hline $\begin{array}{l}\text { C1 pdr1 } \Delta:: \text { natR confirmation primer forward } \\
\text { external }\end{array}$ & GCAGGACCATAGCGGCCA \\
\hline $\begin{array}{l}\text { C2 pdr1 } 1: \text { :natR confirmation primer reverse } \\
\text { internal }\end{array}$ & TACGAGACGACCACGAAGC \\
\hline $\begin{array}{l}\text { C3 pdr1 } \Delta:: \text { natR confirmation primer forward } \\
\text { internal }\end{array}$ & TGGAACCGCCGGCTGACC \\
\hline $\begin{array}{l}\text { C4 pdr1 } 1:: \text { natR confirmation primer reverse } \\
\text { external }\end{array}$ & CGCCTTTACTGGTGGGCC \\
\hline $\begin{array}{l}\text { C1 pdr3 } \triangle:: U R A 3 \text { confirmation primer forward } \\
\text { external }\end{array}$ & TACCGCCTAGGTAACCAT \\
\hline $\begin{array}{l}\text { C2 pdr3 } \triangle:: U R A 3 \text { confirmation primer reverse } \\
\text { internal }\end{array}$ & AATTCAACGCGTCTGTGAGG \\
\hline $\begin{array}{l}\text { C3 pdr3 } \triangle:: U R A 3 \text { confirmation primer forward } \\
\text { internal }\end{array}$ & GACACCTGGAGTTGGATT \\
\hline $\begin{array}{l}\text { C4 pdr3 } \triangle:: U R A 3 \text { confirmation primer reverse } \\
\text { external }\end{array}$ & TTATGAACACGCACAGGC \\
\hline
\end{tabular}

Table 2.3 - Deletion PCR primers. The deletion primers were designed with 50 bp of homology to the $5^{\prime}$ or $3^{\prime}$ flanking regions of PDR1 or PDR3 loci and $18 \mathrm{bp}$ with homology to natR or URA3 cassette. The confirmation primers consist of $18 \mathrm{bp}$ (see Fig. 2.2). All primers were constructed according to Janke et al. (57).

\subsubsection{Yeast transformation}

Yeast strain Y7092 was first transformed with the natR deletion cassette to generate

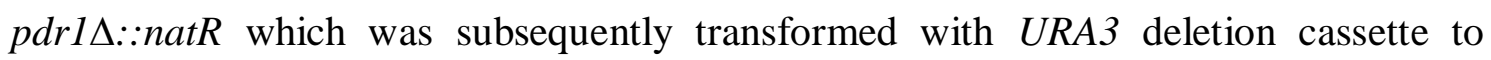
generate $p d r 1 \Delta:: n a t R$ pdr3 $\Delta:: U R A 3$. Yeast transformations were performed according to Gietz \& Schiestl, 2007 (58). 


\subsubsection{Construct confirmation by PCR}

Genomic DNA was harvested from a single colony of $p d r 1 \Delta::$ natR $p d r 3 \Delta:: U R A 3$ strain and $5^{\prime}$ and $3^{\prime}$ genomics flanking regions of the deletion cassettes were amplified using HotStarTaq PCR amplification kit (Qiagen, cat. 203205) using the strategy illustrated in Fig. 2.2. The colony PCR was modified from Amberg et al. (59) and the reagents and reaction volumes are given in Table 2.4 .

The reaction conditions were as follows: $94^{\circ} \mathrm{C}$ for 15 min for HotStarTaq activation and template denaturation, then 35 cycles of $95^{\circ} \mathrm{C}$ for $45 \mathrm{~s}$ for template denaturation, $60.2^{\circ} \mathrm{C}$ for $40 \mathrm{~s}$ for primer annealing and $68^{\circ} \mathrm{C}$ for $1 \mathrm{~min}$ for primer extension, and finally, $68^{\circ} \mathrm{C}$ for $10 \mathrm{~min}$ more to complete any partial extended primer. Upon completion PCR products were stored at $10^{\circ} \mathrm{C}$ until removed from the machine to prevent heat denaturation. $5 \mu \mathrm{L}$ of PCR products were electrophoresed on an $1 \%$ agarose gel stained with ethidium bromide and were visualised under UV.

\begin{tabular}{|l|l|}
\hline Reagents & Volume $(\mu \mathrm{L})$ \\
\hline $\mathrm{H}_{2} \mathrm{O}$ & 10.15 \\
\hline Q buffer & 5 \\
\hline $10 x$ PCR buffer & 2.5 \\
\hline dNTP (5 mM) & 1 \\
\hline Forward deletion primer $(100 \mathrm{mM})$ & 0.25 \\
\hline Reverse deletion primer $(100 \mathrm{mM})$ & 0.25 \\
\hline HotStarTaq & 0.1 \\
\hline Template & 4 \\
\hline Total & 25 \\
\hline
\end{tabular}

Table 2.4 - PCR reagents and volumes for construct confirmation PCR. 
a

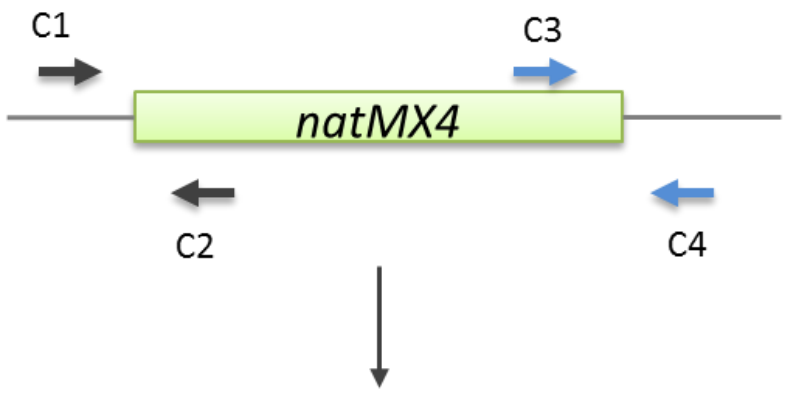

b

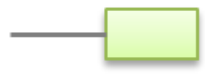

Deletion cassette (natR or URA3) and confirmation primers: $\mathrm{C} 1$ external forward primer, $\mathrm{C} 2$ reverse internal primer, $\mathrm{C} 3$ internal forward primer and $\mathrm{C} 4$ reverse external primer.

Resulting PCR products

Figure 2.2 - Constructions of confirmation primers. (a) Above is a diagrammatic representation of the strategy used to confirm the correct insertion and orientation of natR and URA3 deletion cassettes into respective PDR1 and PDR3 loci. 18 bp confirmation primers were used to amplify sequences overlapping the $5^{\prime}$ and $3^{\prime}$ flanking regions of the deletion cassettes. Direction of the arrows designates the direction of primer extension. Primers denoted as C1 and C2, C3 and C4 (sequences given in Table 2.3) were used as forward and reverse primer pairs for each confirmation PCR reaction. (b) The resulting PCR products.

\subsubsection{Functional confirmation}

The loss of PDR1 and PDR3 loci from the wild-type background was confirmed by treatment of wild-type (Y7092), $\Delta p d r 1$ and , $\Delta p d r 1 \Delta p d r 3$ with the growth inhibitory compounds cycloheximide and rapamycin in a dose response assay.

\subsubsection{Dose response assay}

Starting at an initial concentration of $100 \mu \mathrm{M}$, serial dilutions in half- $\log (\sqrt{ } 10 \approx 3.162$ fold) decrements were made for each inhibitory compound by dilution in the carrier solvent DMSO (dimethyl sulfoxide). The serial dilutions were transferred to wells in a 96 well V-bottomed plate (KJ511-3, Interlab) along with a DMSO alone for the 
negative control to determine the residual growth for each yeast strain in the absence of growth inhibitory compound.

A single colony of yeast strain of interest was inoculated into $3 \mathrm{~mL}$ of synthetic complete (SC) broth with the appropriate antibiotics and was incubated on a rotating drum at $30^{\circ} \mathrm{C}$ and $40 \mathrm{rpm}$ for $16 \mathrm{~h}$. Cell titre was determined using a haemocytometer and $5 \times 10^{5}$ cells $/ \mathrm{mL}$ were inoculated into $10 \mathrm{~mL}$ of SC broth for each yeast strain and vortexed. $99 \mu \mathrm{L}$ of SC plus cells mixture were delivered into the wells of an 96 well flat bottomed plate (TCP-000-096, JET BIOFIL) using a multichannel pipette, for each strain in each drug condition in triplicate. Subsequently, $1 \mu \mathrm{L}$ of half log drug dilutions and DMSO were added to the yeast cells. A row of SC broth alone controls were included for each plate to determine the optical density (OD) of the growth media and sterility. The contents of the wells were mixed by vortexing the plates at $1000 \mathrm{rpm}$ on for $30 \mathrm{~s}$ using the Eppendorf Mix Mate (Krackeler Scientific Inc. Albany, New York) and were incubated at $30^{\circ} \mathrm{C}$ for $17 \mathrm{~h}$.

The cultures were resuspended by vortexing at $1000 \mathrm{rpm}$ for $30 \mathrm{~s}$ and the OD in each well was measured at $590 \mathrm{~nm}$ using Envision ${ }^{\mathrm{TM}}$ Xcite $^{\mathrm{TM}} 2102$ Multilabel Reader (Perkin-Elmer, Turku, Finland) with HTS microplate reader software version 1.12. The OD in each well was subtracted by the average OD for media alone control. The residual growth $\left(\mathrm{OD}_{590} \mathrm{Exp} / \mathrm{OD}_{590} \mathrm{DMSO}\right.$ control $\left.\mathrm{x} 100\right)$ was determined and the average residual growth plotted using Microsoft Excel. 


\subsubsection{Synthetic Genetic Array (SGA)}

SGA analysis was adapted from Tong \& Boone, 2006 (51) to create a DMA containing the background of null mutations in the PDRI and PDR3 transcription factors. The haploid DMA was obtained as a gift from Charles Boone, University of Toronto which contains strains comprising all the non-essential single gene deletions (minus slowgrowing strains) replaced with a kanR deletion cassette. The DMA was maintained as haploid MATa mutants in 1536 colonies per plate format on 14 plates. Each plate contained 384 strains in quadruplicate with a his $3 \Delta:: k a n R$ control strain border comprising 144 colonies along the edge of the plate. Since SGA results rely on assessment of colony sizes, the his $3 \Delta:: k a n R$ control border at the edge of the plate ensures the experimental colony size is not biased because the colonies at the edge grow larger having more access to media. The his $3 \Delta:: k a n R$ was also used to control variation in colony size between plates. The his $3 \Delta:: k a n R$ does not show any genetic interaction with any query mutations since histidine prototrophy is achieved via can1 $\mathrm{S}:$ STE2pr$s p$-HIS5 in the recombinant MATa strains. The replica pinning was performed using the singer RoToR HDA (Singer Instrument Co. Ltd, Somerset, UK).

\subsubsection{Synthetic Genetic Array methodology (see Fig. 2.3)}

\section{Mating the query with DMA:}

The $p d r 1 \Delta::$ natR $p d r 3 \Delta:: U R A 3$ double mutant was grown in $5 \mathrm{~mL}$ of SD-Ura + clonNAT broth at $30^{\circ} \mathrm{C}$ and $40 \mathrm{rpm}$ overnight. The culture was poured over an YPD plate. Using the 96 long pin RoToR pads, YPD plates were pinned in an array 4 times for each plate to generate the query in 384 format and were incubated at $30^{\circ} \mathrm{C}$ for $1 \mathrm{~d}$. 
The 384 format plates were indexed in a square pattern in an array 4 times for each plate using 384 pin RoToR pads generating the 1536 format and incubated at $30^{\circ} \mathrm{C}$ for 1

d. The query strain in 1536 format was replica pinned onto 14 fresh YPD plates. The DMA in 1536 format was pinned on top of the query strain and the plates were incubated at $30^{\circ} \mathrm{C}$ for $1 \mathrm{~d}$ to initiate mating.

\section{$M A T a / \alpha$ zygotes selection and sporulation:}

The MATa/ $\alpha$ zygotes were pinned onto SD-Ura $+\mathrm{G} 418$ plates and incubated at $30^{\circ} \mathrm{C}$ for $2 \mathrm{~d}$ to select for heterozygous diploids containing the genetic material of both haploids. SD-Ura selects for the $U R A 3$ prototrophy cassette linked to $\Delta p d r 3$ mutation thereby selecting for the query strain genome while the antibiotic G418 selects for kanR cassette linked to the gene deletion mutation thereby selecting for the DMA genome.

The resultant diploids were pinned onto enriched sporulation media plates and were incubated at $25^{\circ} \mathrm{C}$ for $5 \mathrm{~d}$. Enriched sporulation media contains low source of carbon and nitrogen. The limited nutrients and incubation at $25^{\circ} \mathrm{C}$ induces the sporulation of diploid. The resulting spores are a collection of wild-type, single, double, and triple mutants for the $p d r 1 \Delta:: n a t R, p d r 3 \Delta:: U R A 3$ and $x x x \Delta:: k a n R$ loci due to recombination and independent assortment of chromosomes. A series of pinning steps onto selective media is utilised to isolate the desired strains. 


\section{MATa selection:}

Meiotic progeny were pinned on to SD - His/Arg/Lys + canavanine/thialysine plates and were incubated at $30^{\circ} \mathrm{C}$ for $2 \mathrm{~d}$. Incubating in media lacking histidine selects for MATa meiotic progeny expressing STE2pr-Sp_HIS5. STE2pr-Sp_HIS5 is a HIS5 gene from $S$. pombi under the control of the STE2 promoter of S. cerevisiae. The STE2 gene encodes for the alpha factor hormone receptor which is only expressed in MATa mating type cells (60). Therefore, Sp_HIS5 under the STE2 promoter only confers histidine

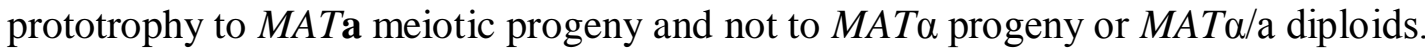

The S. pombi HIS5 was used as a dominant selectable marker since the his $3 \Delta l$ mutation carried by parental strains is only a partial deletion of HIS3 and parental strains can become HIS3 revertants through gene conversion following homologous recombination with a $S$. cerevisiae HIS3 selectable marker (55). The sequence discrepancy present in $s p_{-} H I S 5$ prevents homologous recombination with the his $3 \Delta l$ deletion and allows the selection of true MATa meiotic progeny(55).

In the rare event where recombination occurs in the homologous regions between the MAT locus and the centromere region on chromosome III, gene conversion can result in

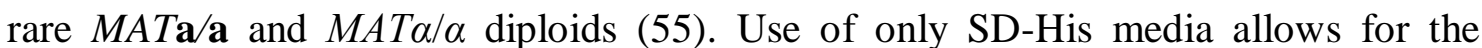
selection of MATa/a diploids leading to false negatives on SGA. To overcome such complications with MATa/a diploids, the use of canavanine and thialysine counterselections were used. 
The wild-type CAN1 and LYPI loci carried by the DMA MATa $x x x \Delta:: k a n R$ strains encode an arginine permease and lysine permease respectively. The arginine permease in addition to importing arginine permits the entry of canavanine, a toxic analog of arginine, into the cell leading to cell death. Similarly, the lysine permease permits entry to thialysine, a toxic analog of lysine, which also causes cell death. Growth of spores in media containing canavanine and thialysine; and lacking arginine and lysine, selects for meiotic progeny carrying the $\Delta c a n l$ and $\Delta l y p l$ mutations. More importantly, this selection kills the MATa/ $\alpha$ diploids since they carry the wild-type CAN1 and LYP1 loci allowing entry to canavanine and thialysine. Media lacking arginine and lysine were used for selection of MATa progeny because the presence of the arginine and lysine amino acids competitively inhibits the entry of their toxic analogs. Although it is still possible for recombination to also occur in $\Delta c a n 1$ and $\Delta l y p l$ loci; it is very unlikely for 3 independent recombination events (MATa/a, $\Delta$ canl/Acanl and $\Delta l y p 1 / \Delta l y p 1)$ to occur within one cell. 

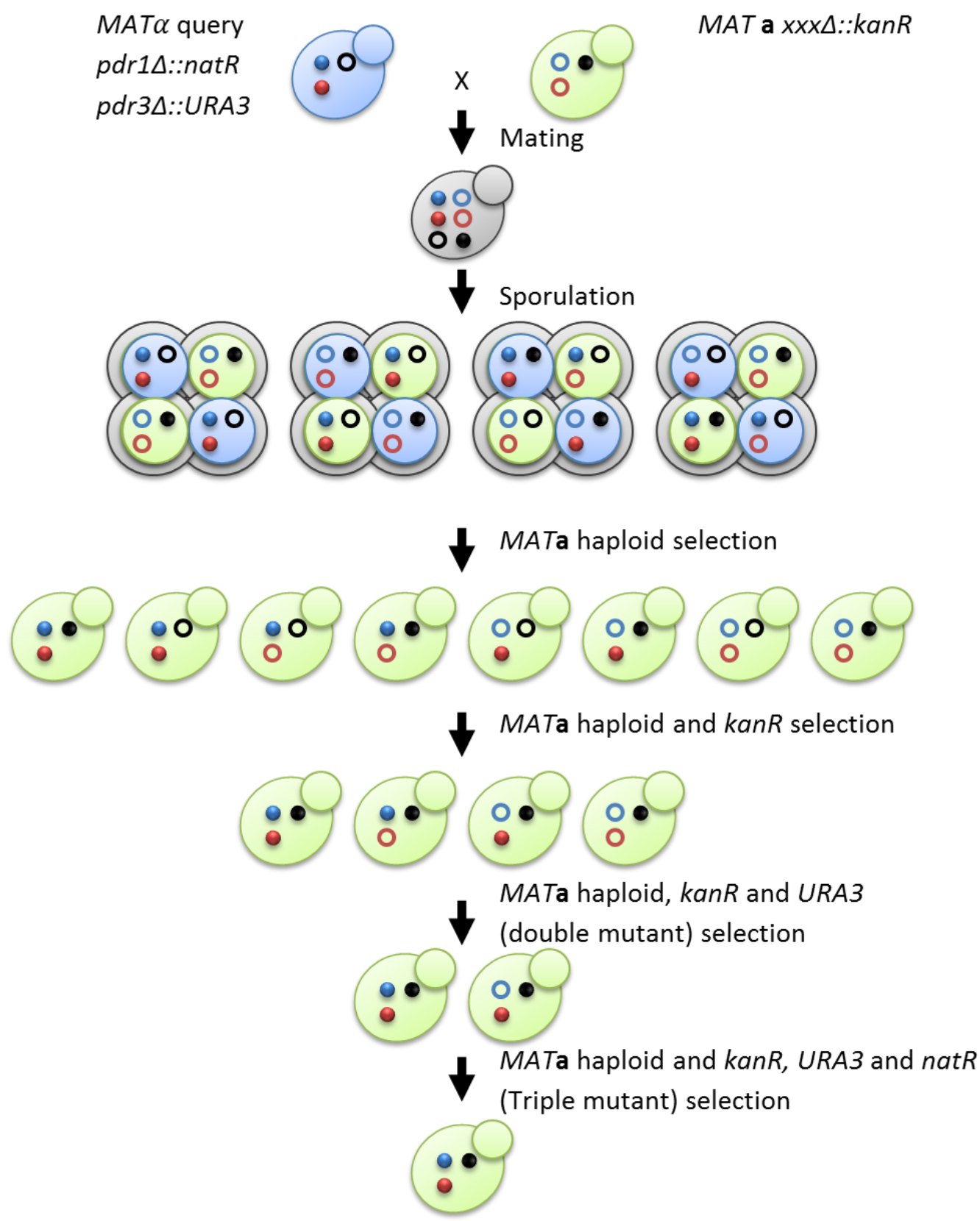

Figure 2.3 - Synthetic generic array methodology (modified from Tong \& Boone, 2005). O PDR1, OPDR3,

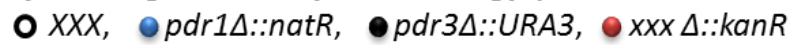

\section{Second MATa selection:}

The MATa meiotic progeny were re-pinned onto SD - His/Arg/Lys + canavanine/thialysine and were incubated at $30^{\circ} \mathrm{C}$ for $1 \mathrm{~d}$ to ensure that the surviving cells are MATa haploids. Only $1 \mathrm{~d}$ growth was necessary the second time since the majority of cells following the first selection were MATa haploids. 


\section{MATa-kanR Meiotic progeny selection:}

MATa meiotic progeny were pinned onto SD-His/Arg/Lys + canavanine/ thialysine/G418 plates and incubated at $30^{\circ} \mathrm{C}$ for $2 \mathrm{~d}$. Media lacking His/Arg/Lys + canavanine/ thialysine maintains the selection pressure for haploid MATa meiotic progeny while the G418 selects for MATa meiotic progeny carrying the kanR marker which is linked to the DMA deletions.

Following MATa-kanR selection MATa meiotic progeny carrying the can $1 \Delta:: k a n R$ deletions are not expected to grow on the MATa-kanR selection plates. The $\Delta$ canl deletion can carry either the kanR or the STE2pr-Sp-HIS5 markers, but not both and both markers are needed for survival on this selection media. The MATa progeny carrying the lyp $1 \Delta:: k a n R$ deletion mutations are expected to grow, since the $\Delta l y p l$ deletion is not linked to a selectable marker and the kanR marker selects for meiotic progeny carrying lypl $1 \Delta:: k a n R$. The genes in close physical proximity to $\Delta$ canl and Slypl loci, called a 'linkage group', display no-growth phenotypes in the presence of antibiotics owing lack of recombination between selection markers leading to linkage disequilibrium. Linkage groups are also expected for strains carrying a gene deletion in close proximity to $\Delta l y p l$ owing to linkage disequilibrium.

\section{MATa-kanR-URA3 Meiotic progeny selection:}

MATa meiotic progeny carrying $x x x \Delta:: k a n R$ mutations were pinned onto SDHis/Arg/Lys/Ura + canavanine/ thialysine/G418 and were incubated at $30^{\circ} \mathrm{C} 2 \mathrm{~d}$. Media lacking His/Arg/Lys + canavanine/ thialysine/G418 maintains the selection pressure for haploid MATa meiotic progeny carrying $x x x \Delta:: k a n R$ deletions. Absence of uracil 
selects for MATa-kanR meiotic progeny carrying the URA3 prototrophic cassette linked to $\Delta p d r 3$ deletion.

\section{MATa-kanR-URA3-natR Meiotic progeny selection:}

MATa meiotic progeny carrying $x x x \Delta:: k a n R$ and $\Delta p d r 3$ deletions are pinned onto SDHis/Arg/Lys/Ura + canavanine/ thialysine/G418/clonNAT plates and were incubated at $30^{\circ} \mathrm{C} 2$ d. Media lacking His/Arg/Lys/Ura + canavanine/ thialysine/G418/clonNAT maintains the selection pressure for haploid MATa meiotic progeny carrying $x x x \Delta:: k a n R$ and $p d r 3 \triangle U R A 3$ deletions. The antibiotic clonNAT selects for MATa meiotic progeny carrying the natR cassette linked to $\Delta p d r l$ deletion. The resulting $p d r l \Delta:: n a t R$ $p d r 3 \Delta:: U R A 3 x x x \Delta:: k a n R$ triple mutants were analysed for fitness by measuring colony size.

\subsubsection{SGA analysis.}

Synthetic lethal and synthetic sick interactions were determined by comparing colony size of the triple mutants to the 2 parental strains. The colony sizes were analysed in high throughput using Colony HT software (61), and uploaded into a MYSQL database containing the colony position of every gene deletion mutant within the DMA.

The colony sizes of the triple mutants were analysed in high throughput using SESA (SGA experiment set analyser) developed by Cameron Jack (SBS, VUW) which utilises statistical algorithms comparing colony size of the experimental set against the control set which comprises 8 independent SGA's. The control sets were conducted using 
ura3A::natR as the starting strain for the SGA using the same growth conditions as the experimental SGA's. The $\Delta p d r 1 \Delta p d r 3$ query strain contains the $\Delta u r a 3$ deletion in its genome, therefore using $\Delta u r a 3$ SGA's as the control set allows to examine the $\Delta p d r 1$ $\Delta p d r 3$ deletion mutations. Use of this control allows for the removal of non-specific genetic interactions, such as linkage groups surrounding $\Delta c a n l$ and $\Delta l y p l$ deletion mutants, and also removes the slow growing yeast strains and strains that fail to sporulate due to deletions in genes that are involved in mating and sporulation.

Plate-to-plate variation is controlled using the ratio of the mutant colony area and comparing against the median control his $3 \Delta:: k a n R$ colony area on each plate. Positional colony growth is controlled by comparing the growth of the triple mutant at a given position against the growth of the double mutant in the $\Delta u r a 3$ control SGA's in the same position. To improve statistical robustness against errors induced by pinning, spontaneous mutations and genetic drift, 3 independent SGAs should be performed. In this thesis only two SGAs were performed since their purpose was to generate a DMA in the $\Delta p d r 1 \Delta p d r 3$ genetic background rather than assessing the genetic interactions with the PDRI and PDR3 loci nevertheless the significant genetic interactions derived from SESA were evaluated. Strains with gene deletions in close physical proximity to the query genes, PDR1 and PDR3, which showed reduced growth were discounted as false positives since they belong to the linkage group of either PDR1 or PDR3 and displayed linkage disequilibrium and were not real genetic interactions. However, such linkage groups surrounding the query ORFs can be used to confirm the query strain contains these genetic alterations since the genes showing linkage disequilibrium should be in close chromosomal position to the query gene. The remaining hits are given in the 
results section with their function categorised according to Saccharomyces Genome Database (SGD) (62).

\subsubsection{Analysis of metabolic pathways}

Metabolic pathway maps were downloaded from Kyoto Encyclopaedia of Genes and Genomes (KEGG) and were used to map the genetic interactions from molecular data sets; particularly, the pyrimidine biosynthesis pathway.

\subsubsection{Confirming putative genetic interactions with $\Delta p d r 1 \Delta p d r 3$ double mutant.}

The putative genetic interactions identified with $\Delta p d r 1 \Delta p d r 3$ deletions were confirmed by random spore analysis. The appropriate gene deletion mutants from the DMA were independently mated against the query strain, pdr1 $1:: n a t R$ pdr3 $3:: U R A 3$ on YPD plates and were incubated at $30^{\circ} \mathrm{C}$ for $1 \mathrm{~d}$. The heterozygous diploids were selected by replica plating onto SD-Ura $+\mathrm{G} 418$ plates and incubated at $30^{\circ} \mathrm{C}$ for $2 \mathrm{~d}$. The diploids were replica plated onto GNA media and were incubated at $30^{\circ} \mathrm{C}$ for $16 \mathrm{~h}$ followed by replica plating onto enrich sporulation media plates and were incubated at $25^{\circ} \mathrm{C}$ for $5 \mathrm{~d}$. The replica pinning of diploids from nutrient rich GNA media to low nutrient enriched sporulation media induces the sporulation of the diploid yeast. The resulting spores are

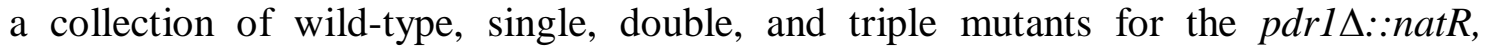
pdr3A::URA3 and $x x x \Delta:: k a n R$ loci due to recombination and independent assortment of chromosomes. 


\subsubsection{Random spore analysis}

The methodology for random spore analysis was adapted from Tong et al. for selection of triple mutants (63). Spores for each diploid strain were inoculated into $50 \mu \mathrm{L}$ volumes of $0.25 \mathrm{mg} / \mathrm{mL}$ Zymolase in $1 \mathrm{M}$ sorbitol in $1.5 \mathrm{~mL}$ microfuge tubes using a tooth pick, vortexed and incubated at $30^{\circ} \mathrm{C}$ for $1 \mathrm{~h}$. Zymolase contains $\beta$-glucuronidase which disrupts the ascus coat releasing the ascospores while sorbitol protects against osmotic lysis. $950 \mu \mathrm{L}$ of $\mathrm{ddH}_{2} \mathrm{O}$ was added to the microfuge tubes to stop the Zymolase digestion and $20 \mu \mathrm{L}$ were spread onto MATa selection (SD - His/Arg/Lys + canavanine/thialysine), $45 \mu \mathrm{L}$ onto each of the single mutant selection MATa $\triangle x x x$ selection (SD - His/Arg/Lys + canavanine/thialysine/G418), MATa $\Delta p d r 1$ selection (SD - His/Arg/Lys + canavanine/thialysine/ClonNAT) and MATa $\Delta p d r 3$ selection (SD His/Arg/Lys/Ura + canavanine/thialysine) plates and $180 \mu \mathrm{L}$ onto MATa $\Delta p d r 1 \Delta p d r 3 \Delta x x x$ triple mutant selection $\quad(\mathrm{SD}-\mathrm{His} / \mathrm{Arg} / \mathrm{Lys} / \mathrm{Ura}+$ canavanine/thialysine/ClonNAT/G418) plates respectively, leading to approximately the same number of colonies on each selection plate in the absence of genetic interactions. The plates were incubated at $30^{\circ} \mathrm{C}$ for $2 \mathrm{~d}$. The size and number of colonies formed under the 5 conditions were assessed using ImageJ analysis software (rsbweb.nih.gov/ij/) and were scored for epistatic interactions in which a synthetic lethal interaction leads to a reduction in colony number and synthetic sick interaction leads to a reduction in colony size for the triple mutants. 
2.2.6 Identifying mating and sporulation defects of single gene deletion mutants.

Appropriate deletion mutants from the DMA were independently mated against ura3s::natR control strain on YPD plates and incubated at $30^{\circ} \mathrm{C}$ for $1 \mathrm{~d}$. Since the $\Delta u r a 3$ mutation is in the genetic background of BY4743, ura3 $\Delta:: n a t R$ strains are phenotypically the same as wild-type and allows to examine the single mutations from the DMA. The heterozygous diploids were selected by replica plating onto YPD + ClonNAT/G418, incubated at $30^{\circ} \mathrm{C}$ for $1 \mathrm{~d}$. Strains with mating defects were identified by the reduced growth of diploids on this media. Heterozygous diploids were replica plated onto GNA plates and incubated at $30^{\circ} \mathrm{C}$ for $16 \mathrm{~h}$ before replica plating onto SPO plates. The diploids were sporulated for $5 \mathrm{~d}$ and sporulation defects were identified by random spore analysis described above.

\subsubsection{Storing PDR-deficient mutants for chemical genetic profiling experiments.}

The final selection of the SGA (SD -His/Arg/Lys/Ura + canavanine/ thialysine/ G418/ clonNAT) was repeated twice more each time incubating at $30^{\circ} \mathrm{C}$ for $1 \mathrm{~d}$. This was to

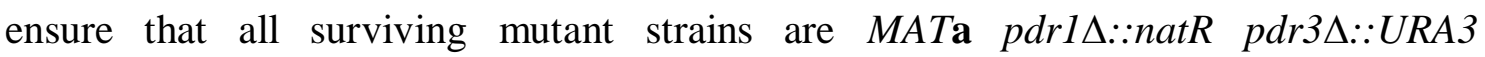
$x x x \Delta:: k a n R$ haploids with similar cell numbers. Three parallel PD-DMA (PD-DMA) sets were generated by pinning onto YPD+G418 plates and were incubated at $30^{\circ} \mathrm{C}$ for $2 \mathrm{~d}$. Two of the PD-DMA sets were inoculated into flat-bottom 384-well plates (Greiner bio-one, cat. 781201 ) containing SC $+25 \%$ glycerol broth using a manual pinner tool and was stored at $-80^{\circ} \mathrm{C}$ as PD-DMA master stocks (59) 
The third PD-DMA was used to generate pools of PD-DMA to be used in barcode microarray experiments. The pdr1 $1:$ natR $p d r 3 \Delta:: U R A 3$ his $3 \Delta:: k a n R$ border was removed from each of the 14 plates prior to pool construction. $5 \mathrm{~mL}$ of YPD $+\mathrm{G} 418$ broth was added on to each plate and the colonies were disrupted off the agar surface using a sterile glass rod. The cell suspension was pipetted into a sterile $500 \mathrm{~mL}$ Schott bottle placed on wet ice. Glycerol was added to a final concentration of $15 \%$, mixed and using a haemocytometer the cell titre determined to be $1 \times 10^{9}$ cells $/ \mathrm{mL}$. In order to obtain 1000x representatives of each of the deletion strains, a minimum of $1 \times 10^{8}$ cells $/ \mathrm{mL}$ was required, as each cycle of freeze thaw halves the number of viable cell. $500 \mu \mathrm{L}$ cell suspensions were aliquoted into $1.5 \mathrm{~mL}$ microfuge tubes and stored at $-80^{\circ} \mathrm{C}$ as PDR-deficient deletion mutant pools (PD-MD) for microarray.

\subsubsection{Genome wide sensitisation of the deletion set to PDR substrates}

Pools of wild-type background DM ( $\Delta x x x)$ (kind gift from Charles Boone, University of Toronto) and the PD-DMA ( $\Delta p d r 1 \Delta p d r 3 \Delta x x x)$ were used in a dose response assay employing the compounds cycloheximide and rapamycin.

\subsubsection{Screening of the LOPAC library}

The commercial library of pharmacologically active compounds (LOPAC) (SigmaAldrich) comprising 1280 compounds were screened against the pools of DMA and PD-DMA to examine if the PDR-deficient strains show increased genome wide sensitivity to compounds. The LOPAC library is maintained in a 96 well format, 
arrayed across 16 plates at a stock concentration of $1 \mathrm{mM}$ in DMSO. In addition, each plate contained a row of controls comprising carrier solvent DMSO alone.

$500 \mathrm{uL}$ of each deletion pool were inoculated into $50 \mathrm{~mL}$ of $\mathrm{SC}$ broth in $250 \mathrm{~mL}$ conical flasks and incubated at $30^{\circ} \mathrm{C}$ and $230 \mathrm{rpm}$ for $16 \mathrm{~h}$. Following incubation, the cell density was determined using a haemocytometer and a fresh $165 \mathrm{~mL}$ of SC broth was inoculated with the deletion pool to a final concentration of $5 \times 10^{5}$ cells $/ \mathrm{mL}$.

$99 \mu \mathrm{L}$ of this cell suspension was delivered into each well of a 96 well flat bottomed plates using a multi-channel pipette. $1 \mu \mathrm{L}$ of each drug in DMSO was delivered into the 96 well flat bottomed plates using the automated $\mathrm{CyBi}{ }^{\circledR}$-well liquid handler (CyBio AG, Jena, Germany) equipped with a pinning tool. A media blank was included on each plate to determine the OD of the growth media. The pins were washed between pinning with methanol and DMSO and were blotted dry to prevent carryover of drugs and cells from one plate to another. The contents of the wells were mixed by vortexing the plates at $1050 \mathrm{rpm}$ for $30 \mathrm{~s}$ on the Eppendorf Mix Mate and were incubated at $30^{\circ} \mathrm{C}$ for $17 \mathrm{~h}$.

The cultures were resuspended by vortexing at $1050 \mathrm{rpm}$ for $30 \mathrm{~s}$ and the OD in each well was measured at $590 \mathrm{~nm}$ using Envision ${ }^{\mathrm{TM}}$ Xcite $^{\mathrm{TM}} 2102$ Multilabel Reader with HTS microplate reader software version 1.12 (PerkinElmer, Massachusetts, USA). Each well was OD corrected by subtracting the average OD of the media alone controls and the residual growth $\left(\mathrm{OD}_{590} \mathrm{Exp} / \mathrm{OD}_{590}\right.$ DMSO control $\left.\times 100\right)$ for each compoundtreated condition was determined. The LOPAC screens for each of the deletion pools 
were performed in triplicate and compounds that were bioactive in all three replicate screens were identified as bioactive.

\subsubsection{Validating hits from the LOPAC library}

The DMA and PD-DMA pools were screened in a dose response assay employing two bioactive compounds from the LOPAC library: camptothecin (Sigma-Aldrich) and ketoconazole (Sigma-Aldrich).

\subsubsection{PD-DMA, media and compounds for chemical genetic profiling in solid phase}

The PD-DMA constructed was maintained as haploid mutant strains in a 1536 colonies per plate format on $14 \mathrm{SC}+\mathrm{G} 418$ plates. Each plate contained 384 strains in

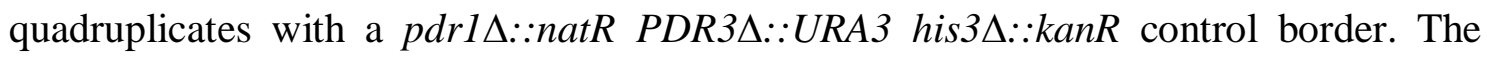

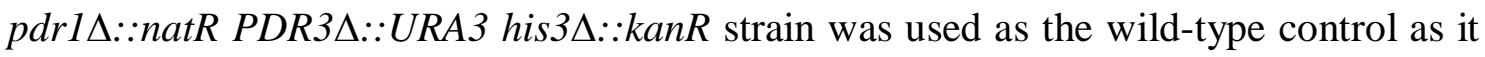
does not show a reduction in growth phenotype due to the presence of $S p \_H I S 5$ which causes histidine prototrophy.

Chemical genetic profiling was performed on SC media as it is chemically defined unlike YPD media which contains various soluble peptides, amino acids and vitamins and can interfere with a given compound's activity. All compounds were dissolved in the DMSO, and added to SC media to a final concentration of $0.1 \%$ DMSO. 


\subsubsection{Identifying $\mathrm{IC}_{\underline{30}}$ in solid phase}

The concentration of the compound which inhibits growth of the PD-DMA by 20-30\% was determined by pinning one of the 14 PD-DMA plates selected at random onto SC plates containing the inhibitory compound across a range of concentration or DMSO alone. The plates were incubated at $30^{\circ} \mathrm{C}$ for $24 \mathrm{~h}$ and the colony size was analysed in high throughput by Colony HT. Residual growth \% for each concentration of compound was determined using the following equation; median $\Delta h i s 3 \Delta p d r l \Delta p d r 3$ border colony size for compound-treated / median $\Delta h i s 3 \Delta p d r 1 \Delta p d r 3$ border colony size for DMSO treated $\times 100$ and the level of inhibition (100-Residual growth\%) was determined relative to growth on $\mathrm{SC}+0.1 \%$ DMSO.

\subsubsection{Chemical genetic profiling in solid phase.}

The PD-DMA was replica pinned onto $\mathrm{SC}+$ compound at $\mathrm{IC}_{30}$ and $\mathrm{SC}+\mathrm{DMSO}$ alone plates using the Singer RoToR. The plates were incubated at $30^{\circ} \mathrm{C}$ for $24 \mathrm{~h}$ and the colony size was measured by Colony HT. Each experiment and the controls were performed in triplicate. The relative fitness of the deletion mutants were determined by SESA, which compared the fitness of the deletion mutants under compound-treated conditions relative to DMSO treated.

\subsubsection{DNA barcode microarray.}

As an alternative to assessing colony size, the barcodes that are unique to each deletion mutant can be utilised to assess the fitness of each deletion mutant in a mixed population. In the DNA barcode microarray, pools of deletion mutants are grown as 
parallel cultures, one culture treated with the inhibitory compound while the other is the untreated control (Fig. 2.4). Genomic DNA from each of the cultures is extracted and the barcodes are amplified by PCR with fluorescently labelled primers, Cy5 (red) for the compound-treated and Cy3 (green) for the untreated sample. The amplified barcodes are hybridised to a microarray chip containing oligonucleotides that are specific for each unique barcode. The competitive hybridisation of barcodes from the compound-treated sample relative to the untreated sample gives a quantitative result of each deletion mutant's fitness and identifies deletion mutants that are hypersensitive or resistant to a given compound of interest.

\subsubsection{Gene deletion pools, media and compounds for DNA barcode microarray}

The pools of PD-DMA constructed as described above were employed in the barcode microarray experiments. All compounds were dissolved in DMSO and were screened at $\mathrm{IC}_{30}$ against a pool of PD-DMA in SC media.

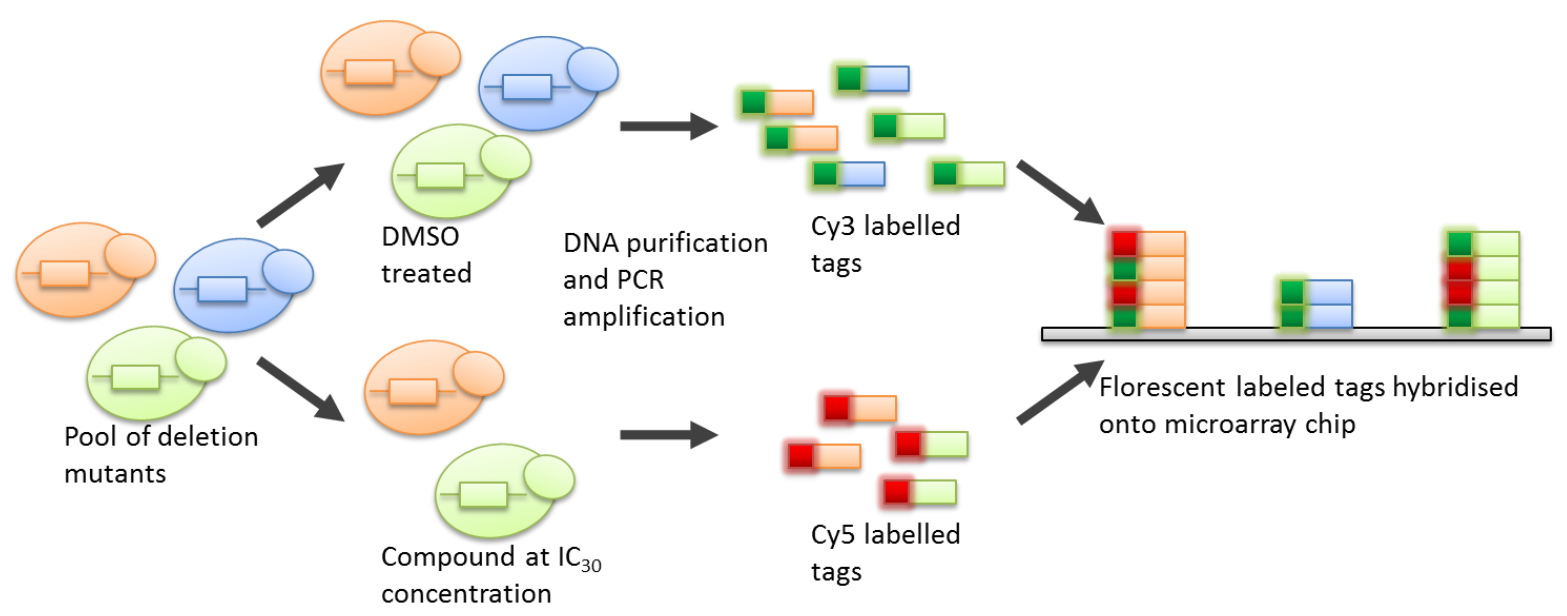


Figure 2.4 - DNA barcode microarray. Yeast deletion mutants each with unique tags are grown in the presence or absence of a growth inhibitory compound. The genomic DNA is extracted and the tags representing each deletion mutant are amplified by PCR with florescent labelled Cy3 and Cy5 primers. The labelled tags are hybridised onto a microarray; the competitive hybridisation leading to a quantitative read-out of each deletion mutant's fitness in the presence of the inhibitory compound and in its absence (adapted from Boone, et al 2007).

\subsubsection{Incubation of cells in compound-treated and control conditions}

DNA barcode microarray experiments were carried out as stated by Cook, 2007 (64). Briefly, $0.5 \mathrm{~mL}$ of the PD-DMA from the $-80^{\circ} \mathrm{C}$ master stock was inoculated into 10 $\mathrm{mL}$ of $\mathrm{SC}+\mathrm{G} 418$ broth and was incubated at $30^{\circ} \mathrm{C}$ overnight on $40 \mathrm{rpm}$. The cell titre was determined using a haemocytometer and two parallel cultures were set up at a cell density of $5 \times 10^{5}$ cells $/ \mathrm{mL}$ in $10 \mathrm{~mL}$ of SC broth. A minimum of $5 \times 10^{6}$ cells were needed to ensure at least 1000 representatives of each mutant strain was contained within the deletion pool to have sufficient coverage of the deletion set. The first of the two parallel cultures was inoculated with $100 \mu \mathrm{L}$ of inhibitory compound to achieve the $\mathrm{IC}_{30}$ concentration while the second culture was inoculated with $100 \mu \mathrm{L}$ of the carrier solvent DMSO alone. Both cultures were incubated at $30^{\circ} \mathrm{C}$ and $40 \mathrm{rpm}$ for $15 \mathrm{~h}$, which is approximately 10 generations of growth.

The cell titre in each $10 \mathrm{~mL}$ culture was determined using a haemocytometer and the cultures were re-diluted to a final cell density of $5 \times 10^{5}$ cells $/ \mathrm{ml}$ in $10 \mathrm{~mL}$ of fresh $\mathrm{SC}$ broth containing either the compound of interest or DMSO alone. The cultures were incubated for a second round for $15 \mathrm{~h}$ at $30^{\circ} \mathrm{C}$ and $40 \mathrm{rpm}$. Rediluting the cultures after 10 generations ensures the drug treatment occurs during the exponential phase of 
growth as the cultures reach saturation after $15 \mathrm{~h}$. The treatment with the compound of interest for a total of 20 generations enhances the growth defect observed for sensitive strains leading to an improved signal to noise ratio in subsequent analysis.

\subsubsection{Genomic DNA purification}

Genomic DNA was harvested from $1.5 \mathrm{~mL}$ cell culture from the compound-treated and the untreated conditions using MasterPure ${ }^{\mathrm{TM}}$ Yeast DNA Purification Kit protocol (Cat. MPY80010, EPICENTER® Biotechnologies). Genomic DNA was resuspended in 35 $\mu \mathrm{L}$ TE (Tris HCl/EDTA) buffer and was treated with $1 \mu \mathrm{L}$ of $5 \mu \mathrm{g} / \mu \mathrm{L}$ RNase A at $37^{\circ} \mathrm{C}$ for 30 min. Removal of RNA by the addition of RNase A is necessary as RNA can interferes with the PCR reactions by binding to $\mathrm{Mg}^{2+}$ ions present in the PCR buffer. Following RNase A treatment, the volume was made up to $500 \mu \mathrm{L}$ by adding $\mathrm{ddH}_{2} \mathrm{O}$. $500 \mu \mathrm{L}$ of phenol/chloroform/isoamylalcohol (25:24:1) was added, mixed by vortexing and centrifuged at $16,000 \mathrm{xg}$ for $10 \mathrm{~min}$. The upper aqueous layer was removed and 1 volume of chloroform was added, vortexed and centrifuged at $16,000 \mathrm{xg}$ for $10 \mathrm{~min}$. The genomic DNA was precipitated by adding 2.5 volumes of $96 \%$ Ethanol and 1/10 volume of $3 \mathrm{M}$ sodium acetate ( $\mathrm{pH} 5.3$ ), mixing by inversion and placed at $-20^{\circ} \mathrm{C}$ for 25 min. The genomic DNA was pelleted by centrifuging at $16000 \mathrm{xg}$ for $10 \mathrm{~min}$, and the DNA pellet was washed in $70 \%$ ethanol and centrifuged for a further $5 \mathrm{~min}$. The DNA pellet was resuspended in $35 \mathrm{uL} \mathrm{TE}$ and incubated at $65^{\circ} \mathrm{C}$ for $10 \mathrm{~min}$ for the pellet to completely dissolve. 
The DNA yield was messured with Hoechst 33258 (Sigma-Aldrich DNA Quantitation kit, DNA-QF) according to manufacturers instructions.

\subsubsection{PCR amplification of UP and DN tags with Cy dye labelled primers}

The PCR reactions were set up in a laminar flow hood under sterile conditions with PCR dedicated pipettes and consumables. DNA solutions and PCR products were excluded from the hood to avoid contamination. The light sensitive Cy dye labelled primers were thawed in the dark. The DNA templates for the PCR reactions were added lastly outside the laminar flow.

In the following PCR, Platinum ${ }^{\circledR}$ Taq PCR amplification kit (Invitrogen, cat. 10966034) was used to amplify the UP and DN tags from treated and untreated DNA samples with Cy dye labelled and unlabelled primers (65) (Table 2.6). Four PCR reactions were set up in $200 \mu \mathrm{L}$ PCR tubes using reagents and volumes given in Table 2.7. For each PCR reaction a negative control was included to check for DNA contamination of reagents.

The reaction conditions were as follows; $94^{\circ} \mathrm{C}$ for $3 \mathrm{~min}$ for template denaturation and Platinum ${ }^{\circledR}$ Taq DNA polymerase activation, then 38 cycles of $95^{\circ} \mathrm{C}$ at $30 \mathrm{~s}$ for template denaturation, $50^{\circ} \mathrm{C}$ for $30 \mathrm{~s}$ for primer annealing and $72^{\circ} \mathrm{C}$ for $30 \mathrm{~s}$ for primer extension, and finally, $72^{\circ} \mathrm{C}$ for $5 \mathrm{~min}$ to complete the synthesis of any partially elongated primers. 
Upon completion, the PCR products were held at $10^{\circ} \mathrm{C}$ until removal from the machine to prevent heat denaturation. The PCR products were stored at $4^{\circ} \mathrm{C}$ covered with aluminium foil to prevent degradation of the light sensitive $\mathrm{Cy}$ dye labelled PCR products. A $5 \mu \mathrm{L}$ aliquot of the PCR products were electrophoresed on a $4 \%$ Metaphor agarose gel stained with ethidium bromide along with a 10 bp ladder (Invitrogen), and was visualised under UV.

\begin{tabular}{|l|l|l|l|}
\hline Condition & Barcode & PCR primer & Primer sequence \\
\hline \multirow{3}{*}{$\begin{array}{l}\text { gDNA from } \\
\text { Experimental }\end{array}$} & UP tag PCR forward primer & U1 & 5'-GATGTCCACGAGGTCTCT \\
\cline { 2 - 4 } & UP tag PCR reverse primer & U2 comp-Cy3 & 5'-Cy[3/5]-GTCGACCTGCAGCGTACG \\
\cline { 2 - 4 } & DN tag PCR forward primer & D1 & 5'-CGGTGTCGGTCTCGTAG \\
\cline { 2 - 4 } & DN tag PCR reverse primer & D2 comp-Cy3 & 5'-Cy[3/5]-CGAGCTCGAATTCATCGAT \\
\hline \multirow{2}{*}{$\begin{array}{l}\text { gDNA from } \\
\text { control }\end{array}$} & UP tag PCR forward primer & U1 & 5'-GATGTCCACGAGGTCTCT \\
\cline { 2 - 4 } & DN tag PCR reverse primer & U2 comp-Cy5 & 5'-Cy[3/5]-GTCGACCTGCAGCGTACG \\
\cline { 2 - 4 } & UP tag PCR forward primer & D1 & 5'-CGGTGTCGGTCTCGTAG \\
\cline { 2 - 4 } & DN tag PCR reverse primer & D2 comp-Cy5 & 5'-Cy[3/5]-CGAGCTCGAATTCATCGAT \\
\hline
\end{tabular}

Table 2.6 - Cy dye labelled and unlabelled primers.

\begin{tabular}{|l|l|}
\hline Reagents & Reaction volume $(\mu \mathrm{L})$ \\
\hline $\mathrm{H}_{2} \mathrm{O}$ & 40.8 \\
\hline $10 \times$ PCR buffer & 6 \\
\hline dNTPs (each $5 \mathrm{mM})$ & 2.4 \\
\hline Labelled primer $(25 \mu \mathrm{M})$ & 2.4 \\
\hline Unlabelled primer $(25 \mu \mathrm{M})$ & 2.4 \\
\hline $\mathrm{MgCl}_{2}(50 \mathrm{mM})$ & 1.8 \\
\hline Platinum taq $(5$ units $/ \mu \mathrm{L})$ & 0.2 \\
\hline DNA $(25 \mathrm{ng} / \mu \mathrm{L})$ & 4 \\
\hline Total & 60 \\
\hline
\end{tabular}

Table 2.7 - PCR reagents and reaction volumes. 


\subsubsection{Precipitation of Cy dye labelled PCR products}

The blocking mix (see Table 2.8) was made using the primers listed in Table 2.9. 55 $\mu \mathrm{L}$ of PCR product from each of the four separate PCR reactions (UP and DN tags for experimental and control PCR reactions) were combined with $20 \mu \mathrm{L}$ of blocking mix, $24 \mu \mathrm{L}$ of $3 \mathrm{M}$ sodium acetate $\mathrm{pH} 5.2,600 \mu \mathrm{L}$ of absolute ethanol and $1 \mu \mathrm{L}$ of $5 \mathrm{ng} / \mathrm{mL}$ linear acrylamide as a carrier for precipitation. The blocking primers consist of oligonucleotide sequences that are complimentary to the common priming sites present on the PCR products and addition of the blocking primers prevent cross hybridisation of the PCR products allowing them to hybridise to the microarray.

The contents was vortexed to mix and incubated at $-20^{\circ} \mathrm{C}$ for $1.25 \mathrm{~h}$ to allow for precipitation then centrifuged at $16000 \mathrm{xg}$ at $4^{\circ} \mathrm{C}$ for $3 \mathrm{~min}$. The DNA pellet was washed with $1 \mathrm{~mL}$ of $70 \%$ ethanol and centrifuged at $4{ }^{\circ} \mathrm{C}$ for a further $5 \mathrm{~min}$. The ethanol was removed and the pellet was dried in the dark at room temperature. The pellet was resuspended in $50 \mu \mathrm{L}$ of dd $22 \mathrm{O}$ and was stored at $-20^{\circ} \mathrm{C}$.

\begin{tabular}{|l|l|}
\hline Primers & Volume $(\mu \mathrm{L})$ \\
\hline U1 $(100 \mu \mathrm{M})$ & 12.5 \\
\hline D1 $(100 \mu \mathrm{M})$ & 12.5 \\
\hline U2 block $(100 \mu \mathrm{M})$ & 12.5 \\
\hline D2 block $(100 \mu \mathrm{M})$ & 12.5 \\
\hline dH20 & 50 \\
\hline
\end{tabular}

Table 2.8 - Blocking mix. 


\begin{tabular}{|l|l|}
\hline Primer & Sequence \\
\hline U1 & 5'-GATGTCCACGAGGTCTCT \\
\hline D1 & 5'-CGGTGTCGGTCTCGTAG \\
\hline D2 & 5'-CGTACGCTGCAGGTCGAC \\
\hline U2 & 5'-ATCGATGAATTCGAGCTCG \\
\hline
\end{tabular}

Table 2.9 - Blocking primer sequences.

\subsubsection{Microarray hybridisation}

2x hybridisation buffer was made (see Table 2.10) and DTT (dithiothreitol) to final concentration of $1 \mathrm{mM}$ was added. DTT prevents oxidation of the fluorescent Cy dyes. $50 \mu \mathrm{L}$ of $2 \mathrm{x}$ hybridisation buffer containing DTT was combined with $50 \mu \mathrm{L}$ of the labelled PCR products containing blocking mix. The sample was mixed by pipetting and was denatured at $95^{\circ} \mathrm{C}$ for 2 min on a heat block and was cooled on ice.

Microarray hybridisation was set up according to the Agilent microarray hybridisation chamber users' guide (Agilent Technologies). The slides were handled by the edges using powder free gloves to prevent artefacts. $100 \mu \mathrm{L}$ of the hybridisation mix was applied to the microarray slide within the hybridisation chamber. The assembled hybridisation chamber was rotated about the vertical axis to completely wet the slide and to mobilise any air bubbles which may have formed when applying the hybridisation mix. The hybridisation chamber was positioned in a vertical orientation in the pre-heated hybridisation oven and incubated at $4 \mathrm{rpm}$ and $42^{\circ} \mathrm{C}$ for $4 \mathrm{~h}$. 


\begin{tabular}{|l|l|}
\hline Reagents & Volume $(\mathbf{m L})$ \\
\hline $5 \mathrm{M} \mathrm{NaCl}$ & 2 \\
\hline $1 \mathrm{M}$ Tris- $\mathrm{HCl} \mathrm{pH7.5}$ & 0.1 \\
\hline Triton $\mathrm{X}-100$ & 0.05 \\
\hline $\mathrm{ddH}_{2} \mathrm{O}$ & 2.85 \\
\hline
\end{tabular}

Table 2.10 - 2X Hybridisation Buffer. Filter sterilised and stored at $4^{\circ} \mathrm{C}$.

\subsubsection{Microarray washing}

20X SSPE solution was made by dissolving the reagents in Table 2.13 in $1 \mathrm{~L} \mathrm{ddH}_{2} \mathrm{O}$ and the $\mathrm{pH}$ was adjusted to 7.4 with $10 \mathrm{M} \mathrm{NaOH}$. $90 \mathrm{~mL}$ 20XSSPE, $210 \mathrm{~mL} \mathrm{ddH}_{2} \mathrm{O}$ and $0.15 \mathrm{~mL}$ Triton $\mathrm{X}-100$ was added to make Wash Solution 1 (6X SSPE+ $0.05 \%$ Triton X-100). $0.18 \mathrm{~mL}$ of 20XSSPE was added to $60 \mathrm{~mL} \mathrm{ddH}_{2} \mathrm{O}$ to make Wash Solution 2 (0.06X SSPE). All wash solutions were filter sterilised through a $0.2 \mu \mathrm{m}$ filter.

The hybridisation chamber was disassembled while submerged in $230 \mathrm{~mL}$ of Wash Solution 1. The microarray slide was transferred into a $50 \mathrm{ml}$ Falcon tube containing 50 $\mathrm{mL}$ of Wash Solution 1 and the slide was dipped up and down 5 times while keeping the arrays submerged under the wash solution. The microarray slide was then transferred to a $50 \mathrm{ml}$ Falcon tube containing $50 \mathrm{~mL}$ of Wash Solution 2 and was washed as described above. The microarray slide was placed in a $50 \mathrm{ml}$ Falcon tube and centrifuged at $500 \mathrm{rpm}$ for $2 \mathrm{~min}$ to remove residual Wash Solution and then air dried in a container with silica desiccant overnight in a dark room. 


\begin{tabular}{|c|c|}
\hline Reagents & Weight (g) \\
\hline $3 \mathrm{M} \mathrm{NaCl}$ & 175.35 \\
\hline $0.2 \mathrm{M} \mathrm{NaH} 2 \mathrm{PO} 4$ (anhydrous, MW $120 \mathrm{~g} / \mathrm{mol}$ ) & 24 \\
\hline $20 \mathrm{mM}$ EDTA. $2 \mathrm{H}_{2} \mathrm{O}$ & 7.4 \\
\hline
\end{tabular}

Table 2.13 - 20X SSPE solution.

\section{$\underline{\text { 2.5.9 Microarray analysis }}$}

The microarray slide was scanned with a Genepix ${ }^{\circledR}$ 4000B microarray scanner situated at the Otago genomics facility (University of Otago, Dunedin, New Zealand). Images of the microarray slides were analysed in Genepix pro 6.0 microarray image analysis software (Molecular Devises, Sunnyvale, USA). Each spot on the array was assigned with a UP or a DN tag specific to a gene deletion mutant. The florescence data for control spots and spots with poor florescence were excluded from further analysis. The remaining data was corrected for intensity dependent bias in $\mathrm{Cy} 5 / \mathrm{Cy} 3$ ratios using the web-based software SNOMAD (66) and spots with poor Cy3 (untreated control) florescence were removed. SNOMAD-normalised log ratios for replicate tags were averaged and $\mathrm{Z}$ scores $((\mathrm{x}-\mu) / \sigma)$ were calculated. Deletion mutants with corresponding UP and DN tags with SNOMAD-normalised Z scores smaller than -3.0 (3 standard deviations below the mean) were identified as hypersensitive strains against a given condition. 


\subsubsection{Validation of hits from microarrays.}

The deletion strains hypersensitivity to a growth inhibitory compound in a DNA barcode microarray were independently validated in a growth assay (see dose response assay), at a single compound concentration, i.e. $\mathrm{IC}_{30}$ for $\Delta p d r 1 \Delta p d r 3 \Delta$ his3 (wild-type control) strain. The appropriate PDR-deficient mutants and $\Delta p d r 1 \Delta p d r 3 \Delta$ his 3 control were treated with either the compound or DMSO alone in triplicate and the plates were incubated at $30^{\circ} \mathrm{C}$. Staring at $12 \mathrm{~h}$, the $\mathrm{OD}$ was measured at $1 \mathrm{~h}$ intervals. For each deletion strain, $\mathrm{OD}_{590} \mathrm{DMSO}$ nearest to 0.3 and its corresponding $\mathrm{OD}_{590}$ compound were used to calculate residual growth $\left(\mathrm{OD}_{590}\right.$ compound/OD $\left.{ }_{590} \mathrm{DMSO} \times 100\right)$. The deletion strains with a greater fitness defect than the $\Delta h i s 3 \Delta p d r l \Delta p d r 3$ control to the compound were determined ( $\Delta x x x$ residual growth $/ \Delta p d r 1 \Delta p d r 3 \Delta$ his3 residual growth $=<0.95$ ). $0.3 \mathrm{OD}_{590} \mathrm{DMSO}$ was used as it is still mid log and reaches saturation at $0.4 \mathrm{OD}$.

\subsection{Network diagram generation}

The results from DNA barcode microarray and solid phase Chemical genetic profiling assays were compiled into network diagrams using Cytoscape (67). Cytoscape is an open source bioinformatics software platform for visualising and integrating molecular interaction networks which uses a Java based plug-in, BiNGO (68). BiNGO maps the gene ontology (GO) categories that are statistically over represented for set of imported genes and out-puts the map as a connected graph. GO is a controlled vocabulary of terms that defines the biology of a gene product in terms of its biological process, molecular function and cellular component and allows clustering of genes by description (69). 
The latest GO annotations and associations were downloaded from http://www.geneontology.org (69). The PD-DMA was used as the reference set to limit the GO annotations only to the deletion mutants present in the DNA barcode microarray and solid phase chemical genetic profiling assays. Gene enrichment in terms of biological process, molecular function and cellular location were assessed for each gene set and fold enrichment ((number genes belonging to the GO term from the gene set/ number of gene belonging to the GO term from the DMA)/ (total number of genes in the gene set/ total number of genes in the DMA)) was determined.

\subsubsection{Image acquisition for confocal microscopy}

A single colony of each GFP strain was inoculated into $3 \mathrm{~mL}$ of SC broth and was incubated at $30{ }^{\circ} \mathrm{C}$ and $40 \mathrm{rpm}$ overnight. Cell titre was determined using a haemocytometer and $10 \mathrm{~mL}$ of fresh $\mathrm{SC}$ broth was inoculated at $5 \times 10^{7}$ cells $/ \mathrm{mL}$ and $49.5 \mu \mathrm{L}$ volumes of the cell suspension was inoculated into each well of a 384 well clear bottomed microliter plate (Perkin Elmer cell carrier) along with $0.5 \mu \mathrm{L}$ of compound or DMSO. Each condition was performed in duplicate. The plates were vortexed at $2000 \mathrm{rpm}$ for $30 \mathrm{~s}$ and were incubated at $30^{\circ} \mathrm{C}$ for $4 \mathrm{~h}$.

The images were acquired using Evo Tec OPERA (Perkin Elmer) high-throughput spinning disk confocal microscope using the 60x water immersion lens NA 1.2 according to Bircham et al (70). The appropriate midsection out of $5 \mathrm{Z}$ stacks was selected by identifying the midsection with the highest contrast difference between the cells and the background. 


\subsubsection{Yap1p subcellular localisation assay}

Yeast strains $Y A P 1-G F P$ and $Y A P 1-G F P \quad \triangle p d r 1 \Delta p d r 3$ were used in confocal microscopy employing rapamycin, latrunculin $\mathrm{A}$, menadione and $\mathrm{H}_{2} \mathrm{O}_{2}$. Cells were visually identified and Yap1p subcellular localisation was detected by the GFP tagged Yap1p fusion protein. Confocal microscopy was performed immediately after treatment with rapamycin, latrunculin $\mathrm{A}$, menadione or $\mathrm{H}_{2} \mathrm{O}_{2}$ as described above.

\subsubsection{UPR induction assay}

Yeast strains $4 \times U P R E-G F P$ and $4 \times U P R E-G F P \Delta p d r 1 \Delta p d r 3$ were used in confocal microscopy employing unfolded protein response element (UPRE) inducing compounds DTT and tunicamycin. The UPR induction was detected by GFP tagged UPRE fusion construct. The GFP intensity of each cell in each well was measured using Acapella, high content imaging and analysis software (Perkin Elmer) and was used as the averaged GFP intensity for each condition. Confocal microscopy was performed as described above. 


\section{PD-DMA Construction and Validation.}

\section{$\underline{\text { Introduction }}$}

A compound's mechanism of action can be elucidated by the identification of yeast deletion mutants that confer hypersensitivity to the particular compound. However, the biological activities of some compounds are attenuated by the mechanisms of the PDR network. This can result in the necessity for the use of higher concentrations of compounds which can be a major disadvantage when the amount of compound available is limiting as is often the case with natural products. For this reason a DMA comprising all the non-essential deletion mutants in a PDR-deficient background was created as a 'reagent' for drug screening utilising yeast. To date there is no such DMA reported in the literature; therefore, this reagent will benefit the scientific community.

Genome wide hypersensitivity of the DMA to selected compounds was determined by pooling the $\sim 4,700$ triple mutant strains and screening this pool against the LOPAC library of pharmacologically active compounds. The PD-DMA (PD-DMA) was then used in chemical genetic profiling assays; namely solid phase chemical genetic profiling and barcode microarray experiments on the compounds, cycloheximide and rapamycin, which have well characterized chemical genetic profiles. These results validated the PD-DMA as a more drug sensitive genome-wide deletion reagent.

This chapter explored some properties of the $\Delta p d r 1 \Delta p d r 3 \Delta x x x$ strains including synthetic lethalities in triple mutants (there were none) and whether the loss of PDRI and PDR3 loci caused an unfolded protein response through UPRE elements (it does 
not) or induced the cellular oxidative stress response (it does not) since these properties are often important in drug screens. 


\section{$\underline{\text { Results }}$}

The PDR network was disabled by generating null mutants of the master transcriptional regulators $P D R 1$ and $P D R 3$. A PDR-deficient strain comprising of $p d r l \Delta:: n a t R$ and pdr3A::URA3 double deletion mutations was constructed with the selectable makers natR and URA3 in place of the PDR genes. This strain was validated by PCR amplification of $5^{\prime}$ and $3^{\prime}$ genomic flanking regions of the $\operatorname{pdr} 1 \Delta::$ natR and pdr3A::URA3 construct to ensure correct disruption of the PDR I/PDR3 loci. In addition the PDR-deficient strain was validated through increased sensitivity to the known PDR substrate cycloheximide, in a dose response assay.

The PDR-deficient strain, $p d r 1 \Delta:: n a t R$ pdr3 $\Delta:: U R A 3$, was robotically mass mated against the DMA comprising the non-essential single deletion mutants, and put through a series of selection steps to generate genome wide DMA in a PDR-deficient background.. Synthetic sick and synthetic lethal interactions with $\Delta p d r l \Delta p d r 3$ double mutant were determined using Colony HT and SESA and were validated by random spore analysis.

\subsubsection{Purification of p4339 and pAG60 plasmids.}

The construction of the PDR-deficient strain $p d r 1 \Delta:: n a t R$ pdr3 $\Delta:: U R A 3$ required the use of the bacterial plasmids pAG60 and p4339 which carry the coding sequence for the selection markers URA3 and natR. The p4339 and pAG60 plasmids were maintained in DH5 $\alpha$ bacterial cells and the plasmids were purified using Zyppy $^{\mathbf{T M}}$ plasmid mini prep kit. The eluted p4339 and pAG60 plasmids were electrophoresed on an 1\% agarose gel 
stained with ethidium bromide and visualised under UV light (Fig. 3.1). The presence of bands for p4339 and pAG60 plasmids on the $1 \%$ agarose gel indicates the plasmid purification was successful. The bands observed for the plasmids p4339 (5086 bp) and pAG60 (3944 bp) appeared less than actual size owing to DNA supercoiling of the plasmids. The p4339 and pAG60 plasmids were used as templates in the PCR amplification of respective natR and URA3 deletion cassettes.

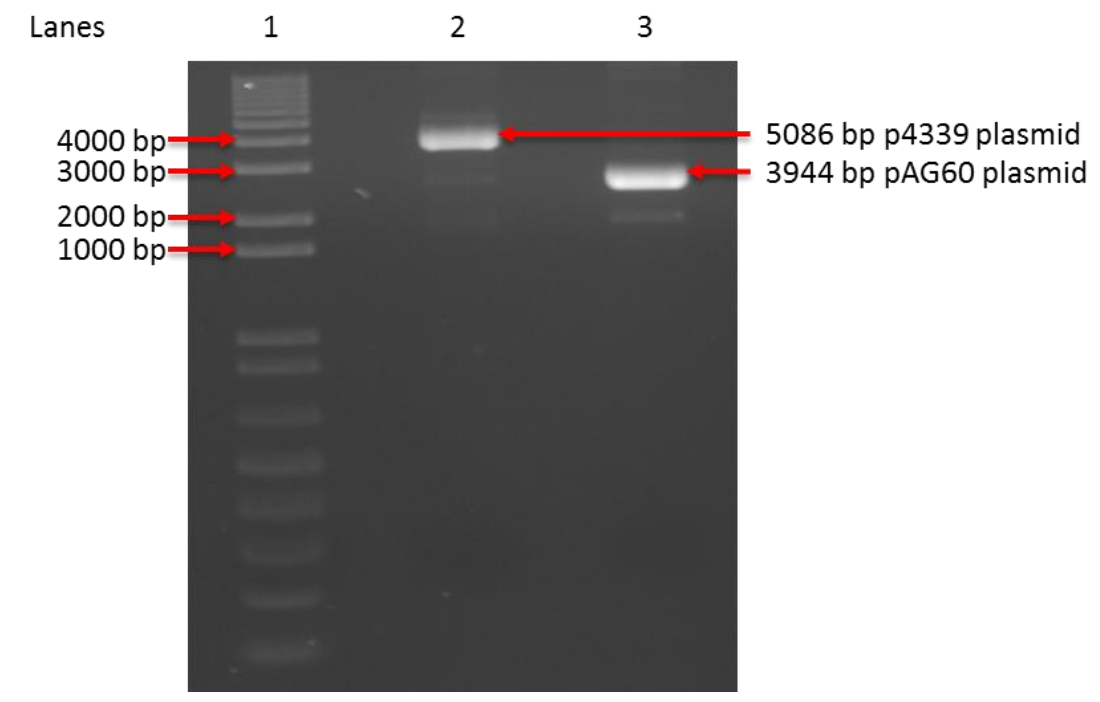

Figure 3.1 - Confirmation of P4339 and pAG60 plasmid isolation. Samples were electrophoresed on $1 \%$ agarose gel. Lane 1. 1-kb plus DNA ladder, Lane 2. Purified p4339 plasmid, Lane 3. Purified pAG60 plasmid. 


\subsubsection{Deletion cassette construction}

To construct the natR and URA3 deletion cassettes, natMX4 and CaURAMX4 cassettes from plasmids p4339 and pAG60 were used as the template with the appropriate deletion primer pairs with sequence homology to flanking region of the selectable marker to permit amplification of the cassettes and sequence homology to the flanking region of the genomic PDRI or PDR3 ORF to promote genomic integration. The deletion cassette was amplified in a gradient PCR using the reaction conditions (Chapter 2 Materials and Methods). The resulting PCR products were electrophoresed on an $1 \%$ agarose gel stained with ethidium bromide and visualised under UV light (Fig. 3.2a -b). The PCR product observed for the natR deletion cassettes was the expected size of $1209 \mathrm{bp}$; similarly, the PCR product observed for the URA3 deletion cassettes was the expected size of $1449 \mathrm{bp}$, indicating that both deletion cassette constructions was successful.

The natR deletion cassette was used firstly to transform the strain Y7092 to create a

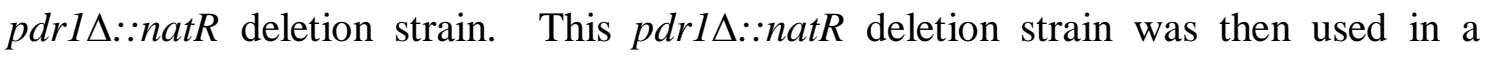
subsequent transformation employing the $U R A 3$ deletion cassette to create the strain

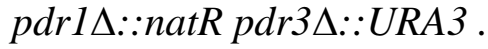


a

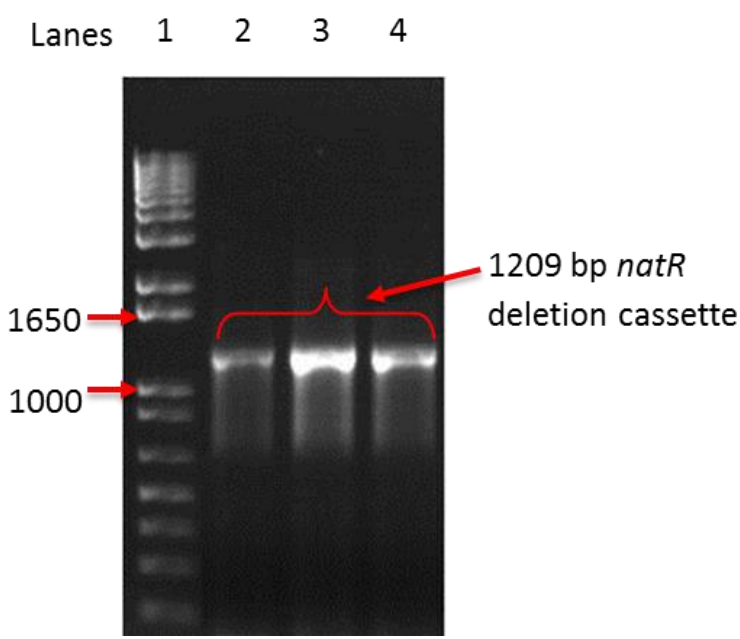

b

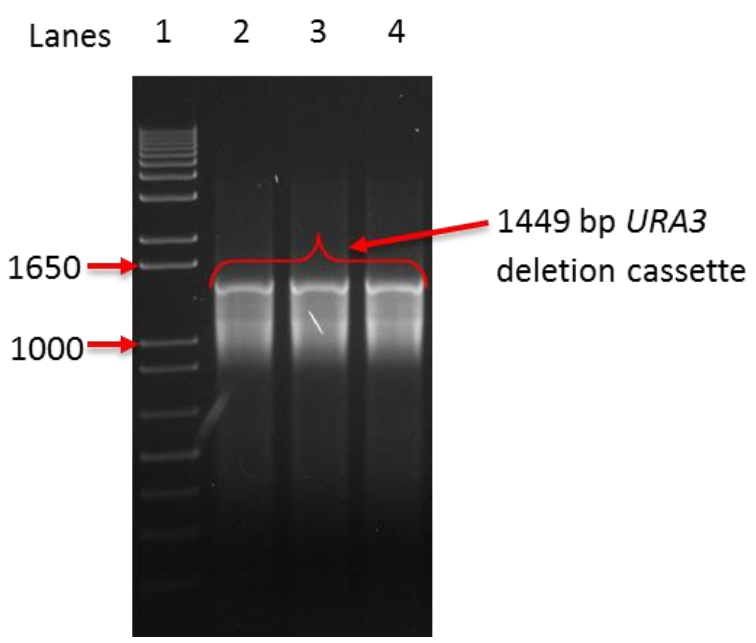

Figure 3.2 - Construction of the natR and URA3 deletion cassettes. PCR products were electrophoresed on an $1 \%$ agarose gel. (a) natR deletion cassette amplification. Lane 1. 1-kb plus DNA ladder, Lane 2. natR deletion cassette using $56.2^{\circ} \mathrm{C}$ annealing temperature, Lane 3 . natR deletion cassette using $60.2^{\circ} \mathrm{C}$ annealing temperature, Lane 4 . natR deletion cassette using $63.5^{\circ} \mathrm{C}$ annealing temperature. (b) URA3 deletion cassette amplification. Lane 1. 1-kb plus DNA ladder, lanes 2. URA3 deletion cassette using $59.4^{\circ} \mathrm{C}$ annealing temperature, Lane 3. URA3 deletion cassette using $60.2^{\circ} \mathrm{C}$ annealing temperature, Lane 4 . URA3 deletion cassette using $63^{\circ} \mathrm{C}$ annealing temperature.

\section{$\underline{\text { 3.1.3 Construct confirmation by PCR }}$}

Colony PCR was performed on the $p d r 1 \Delta:: n a t R$ pdr3 $3:: U R A 3$ transformants selected on SD-uracil and containing clonNAT to confirm the correct integration of the deletion cassettes. Primer pairs were designed such that one PCR primer is internal to the natR and URA3 deletion cassettes while the other PCR primer is in the flanking genomic DNA of the expected integration site. The resulting PCR products were electrophoresed on an $1 \%$ agarose gel stained with ethidium bromide and visualised under UV light (Fig. 3.3). PCR products of the expected size of $761 \mathrm{bp}$ and $880 \mathrm{bp}$ were observed for the PCR reactions amplifying the 5' and 3'flanking region, respectively, of the URA3 
deletion cassette. This confirms the correct integration of the $p d r 3 \Delta:: U R A 3$ construct. Similarly, PCR products of 841 bp and 769 bp were observed for the PCR reactions amplifying either the 3' and 5' flanking region, respectively, of natR deletion cassette confirming the correct integration of $p d r l \Delta::$ natR construct.

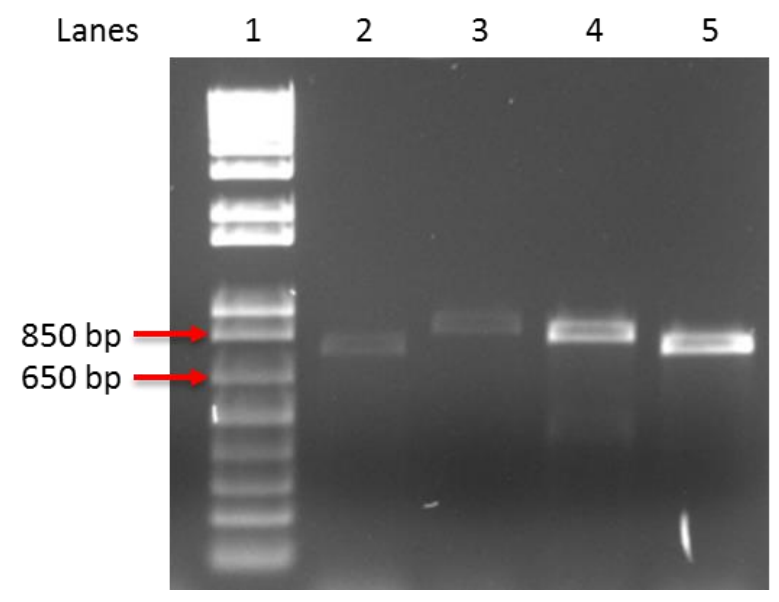

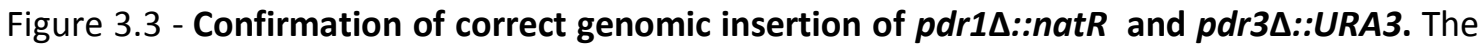
PCR products were electrophoresed on an $1 \%$ agarose gel. Lane 1. 1-kb plus DNA ladder, Lane 2. URA3 deletion cassette $5^{\prime}$ flanking PCR product, Lane 3. URA3 deletion cassette $3^{\prime}$ flanking PCR product, Lane 4. natR deletion cassette 3' flanking PCR product, Lane 5. natR deletion cassette 5' flanking PCR product.

\section{$\underline{\text { 3.1.4 Functional confirmation }}$}

The PDR-deficient strain, $\Delta p d r 1 \Delta p d r 3$, was used in a dose response assay employing the growth inhibitory compounds, cycloheximide, which is a substrate for the PDR pumps and rapamycin, which is not. Growth was determined by measuring optical density and was compared against growth of both a wild-type and a $\Delta p d r l$ single mutant strain (Fig. 3.4a-b). These yeast strains differed in their sensitivity to cycloheximide (Fig. 3.4a). The wild-type was the most resistant to cycloheximide with an $\mathrm{IC}_{50}$ of $\sim 122.33 \mathrm{nM}$, while the loss of Pdr1p trasncriptional regulator in the $\Delta p d r 1$ 
strain resulted in an increased sensitivity to cycloheximide $\left(\mathrm{IC}_{50}\right.$ of $\left.\sim 84.43 \mathrm{nM}\right)$. Loss of both Pdr1p and Pdr3p transcriptional regulators in the $\Delta p d r 1 \Delta p d r 3$ strain further sensitised it to cycloheximide $\left(\mathrm{IC}_{50} \sim 53.90 \mathrm{nM}\right)$.

The two yeast strains, $\Delta p d r l$ and $\Delta p d r 1 \Delta p d r 3$, showed rapamycin sensitivities not significantly different to a wild-type yeast strain (Fig. 3.4b). This is expected since rapamycin is not a substrate for the PDR network and as such the sensitivity to rapamycin is unaffected by the loss of one or both Pdr1p and Pdr3p transcription factors.
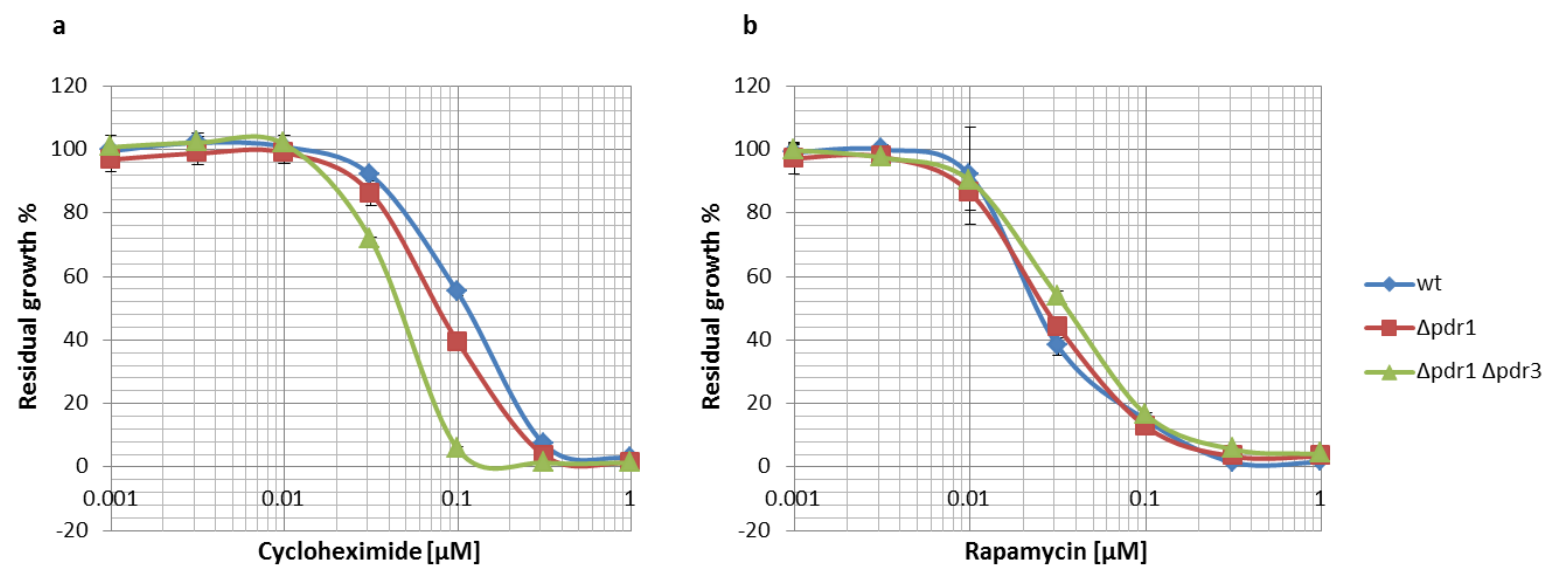

Figure 3.4 - Differential sensitivity to the PDR-substrate cycloheximide. Dose response assays were performed on the yeast strains; wild-type, $\Delta p d r 1$, and $\Delta p d r 1 \Delta p d r 3$, against the $\operatorname{Pdr} 5 p$ substrate cycloheximide and the non-PDR substrate, rapamycin. (a) $\Delta p d r 1 \Delta p d r 3$ was the most sensitive to the PDR substrate, cycloheximide, followed by $\Delta p d r 1$ and wild-type. (b) All three yeast strains had similar sensitivities to the non PDR substrate, rapamycin. 


\subsubsection{SGA analysis}

The PDR-deficient strain $p d r 1 \Delta:: n a t R$ pdr3 $\Delta:: U R A 3$ was robotically mass mated against the non-essential DMA and put through a series of selection steps to generate a genome-wide triple mutant deletion array comprising $\sim 4700$ pdrl $1:$ natR pdr3A::URA3 $x x x \Delta:: k a n R$ mutants. The relative growth rate of each strain was assessed by comparing colonies size utilizing colony HT and SESA image analysis software. Of importance in the SGA procedure (11) is the haploid MATa selection step which is used as described below to differentiate mating efficiency.

Two independent SGAs identified 42 phenotypic enhancements (PE) with the $\Delta p d r 1 / \Delta p d r 3$ double mutant (Table 3.1). The majority of the PEs can be explained by linkage disequilibrium with either the $\Delta p d r l$ or $\Delta p d r 3$ loci where this is readily visible on the SGA plates as a set of contiguous deletions comprising the "linkage group". Nine gene deletion mutants $(\Delta d s f 2, \Delta e c m 15, \Delta f u s 3, \Delta h t a 2, \Delta n t h 2, \Delta p d r 3, \Delta r c r 1, \Delta s l a 1$ and $\Delta u g a 2)$ were in linkage disequilibrium with $\Delta p d r 3$, while17 gene deletion mutants

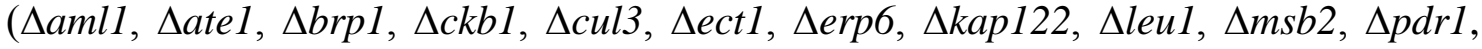
$\Delta p m c 1, \Delta p u f 4, \Delta y g l 010 w, \Delta y g l 015 c, \Delta y g r 016 w$ and $\Delta y g r 018 c)$ were seen in the linkage group of $\Delta p d r 1$. The four genes $U R A 1, U R A 2, U R A 4$ and $U R A 5$ required for pyrimidine biosynthesis were identified as PEs since CaURA3 was used as a selection marker for the $\Delta p d r 3$ deletion. The gene deletion mutants $\Delta m i r 1, \Delta m r m 2, \Delta r a d 14, \Delta r p l 9 a, \Delta s r o 9$, $\Delta y i l 012 w, \Delta y l r 346 c, \Delta y p r 126 c, \Delta z r c 1, \Delta f a r 1, \Delta m f a 1$, and $\Delta$ ste20 seemed to be genuine PEs which needed further investigation for confirmation. 


\begin{tabular}{|c|c|c|c|}
\hline & Gene & ORF & Description \\
\hline \multirow{12}{*}{ 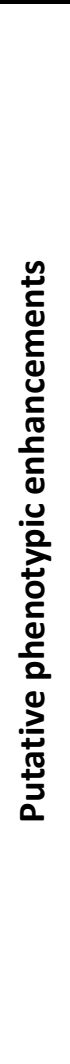 } & MIR1 & YJR077C & Mitochondrial phosphate carrier \\
\hline & MRM2 & YGL136C & Mitochondrial 2' O-ribose methyltransferase \\
\hline & RAD14 & YMR201C & $\begin{array}{l}\text { Protein that recognizes and binds damaged DNA during } \\
\text { nucleotide excision repair }\end{array}$ \\
\hline & RPL9A & YGL147C & Protein component of the large (60S) ribosomal subunit \\
\hline & SRO9 & YCL037C & $\begin{array}{l}\text { RNA-binding protein that associates with translating } \\
\text { ribosomes }\end{array}$ \\
\hline & YIL012W & YIL012W & Dubious ORF but close to YIL013C/PDR11 \\
\hline & YLR346C & YLR346C & Putative protein of unknown function found in mitochondria \\
\hline & YPR126C & YPR126C & Dubious ORF but overlaps YPR125W/YLH47 \\
\hline & ZRC1 & YMR243C & $\begin{array}{l}\text { Vacuolar membrane zinc transporter, transports zinc from } \\
\text { the cytosol }\end{array}$ \\
\hline & FAR1 & YJL157C & Cyclin-dependent kinase inhibitor \\
\hline & MFA1 & YDR461W & Mating pheromone a-factor \\
\hline & STE20 & YHL007C & Cdc42p-activated signal transducing kinase of the PAK family \\
\hline \multirow{9}{*}{ 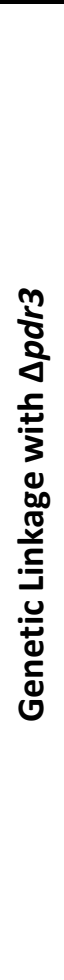 } & DSF2 & YBR007C & Deletion suppressor of $\mathrm{mpt} 5$ mutation \\
\hline & ECM15 & YBL001C & Putative protein of unknown function \\
\hline & FUS3 & YBL016W & $\begin{array}{l}\text { Mitogen-activated serine/threonine protein kinase involved } \\
\text { in mating }\end{array}$ \\
\hline & HTA2 & YBL003C & $\begin{array}{l}\text { Core histone required for chromatin assembly and } \\
\text { chromosome function }\end{array}$ \\
\hline & NTH2 & YBR001C & Putative neutral trehalase \\
\hline & PDR3 & YBL005W & $\begin{array}{l}\text { Transcriptional activator of the pleiotropic drug resistance } \\
\text { network }\end{array}$ \\
\hline & RCR1 & YBR005W & $\begin{array}{l}\text { Protein of the ER membrane involved in cell wall chitin } \\
\text { deposition }\end{array}$ \\
\hline & SLA1 & YBL007C & Cytoskeletal protein binding protein \\
\hline & UGA2 & YBR006W & $\begin{array}{l}\text { Succinate semi-aldehyde dehydrogenase involved in GABA } \\
\text { utilization }\end{array}$ \\
\hline
\end{tabular}




\begin{tabular}{|c|c|c|c|}
\hline \multirow{17}{*}{ 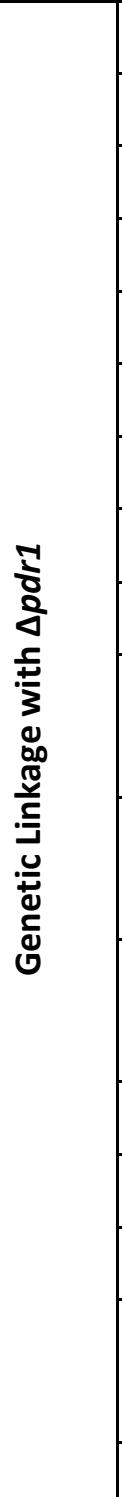 } & AML1 & YGR001C & Putative protein of unknown function \\
\hline & ATE1 & YGL017W & Arginyl-tRNA-protein transferase \\
\hline & BRP1 & YGL007W & Dubious ORF \\
\hline & CKB1 & YGL019W & Beta regulatory subunit of casein kinase 2 (CK2) \\
\hline & CUL3 & YGR003W & Ubiquitin-protein ligase \\
\hline & ECT1 & YGR007W & Choline phosphate cytidylyltransferase \\
\hline & ERP6 & YGL002W & Member of the p24 family involved in ER to Golgi transport \\
\hline & KAP122 & YGL016W & Karyopherin beta \\
\hline & LEU1 & YGLO09C & Isopropylmalate isomerase \\
\hline & MSB2 & YGR014W & $\begin{array}{l}\text { Mucin family member involved in filamentous growth } \\
\text { signaling }\end{array}$ \\
\hline & PDR1 & YGL013C & $\begin{array}{l}\text { Transcription factor that regulates the pleiotropic drug } \\
\text { response }\end{array}$ \\
\hline & PMC1 & YGL006W & $\begin{array}{l}\text { Vacuolar Ca2+ ATPase involved in depleting cytosol of Ca2+ } \\
\text { ions }\end{array}$ \\
\hline & PUF4 & YGL014W & Member of the PUF protein family \\
\hline & YGL010W & YGL010W & Putative protein of unknown function \\
\hline & YGL015C & YGL015C & Putative protein of unknown function \\
\hline & $\begin{array}{l}\text { YGR016 } \\
\text { W }\end{array}$ & YGR016W & Putative protein of unknown function \\
\hline & YGR018C & YGR018C & Protein of unknown function \\
\hline \multirow{4}{*}{ 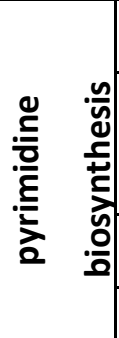 } & URA1 & YKL216W & Dihydroorotate dehydrogenase \\
\hline & URA2 & YJL130C & $\begin{array}{l}\text { Bifunctional carbamoylphosphate synthetase/aspartate } \\
\text { transcarbamylase }\end{array}$ \\
\hline & URA4 & YLR420W & Dihydroorotase \\
\hline & URA5 & YML106W & Major orotate phosphoribosyltransferase (OPRTase) isozyme \\
\hline
\end{tabular}

Table 3.1 - Putative phenotypic enhancements of the PDR-deficient strain. PEs of the $\Delta p d r 1 \Delta p d r 3$ double deletion mutants were identified from 2 independent SGAs. The genes were annotated according to GO function as stated in SGD (62). 


\subsubsection{Random spore analysis}

There were 12 mutant strains that showed apparent PEs with the PDR-deficient strains. They could have shown this phenotype for three reasons: (i) genuine PE epistasis, (ii) low mating efficiency, (iii) low sporulation efficiency. To determine these possibilities they were mated then assessed by random spore analysis. The individual steps in this analysis allow assessment of these three variables.

To assess mating efficiency, the PDR-deficient strain, $\Delta p d r 1 \Delta p d r 3$, was mated against the single DMA deletion mutants identified above by SGA analysis that included 12 mutants at the top of Table 3.1. Analysis of the heterozygous diploids generated determined six deletion mutant strains ( $\Delta$ mirl, $\Delta m r m 2, \Delta \operatorname{rad} 14, \Delta s o r 9, \Delta y l r 346 c$ and $\Delta z r c 1)$ to mate efficiently with the PDR-deficient strain (Fig. 3.5a). In contrast, a significantly reduced number of diploids (low efficiency mating) were observed for crosses involving the PDR-deficient strain and the gene deletion mutants $\Delta$ farl, $\Delta y i l 012 w, \Delta y p r 126 c, \Delta m f a 1, \Delta s t e 20$ and $\Delta r p l 9 a$ in comparison to the $\Delta h i s 3$ control cross where $\Delta m f a l, \Delta$ farland $\Delta$ ste 20 are known low efficiency mating strains (71-73). 

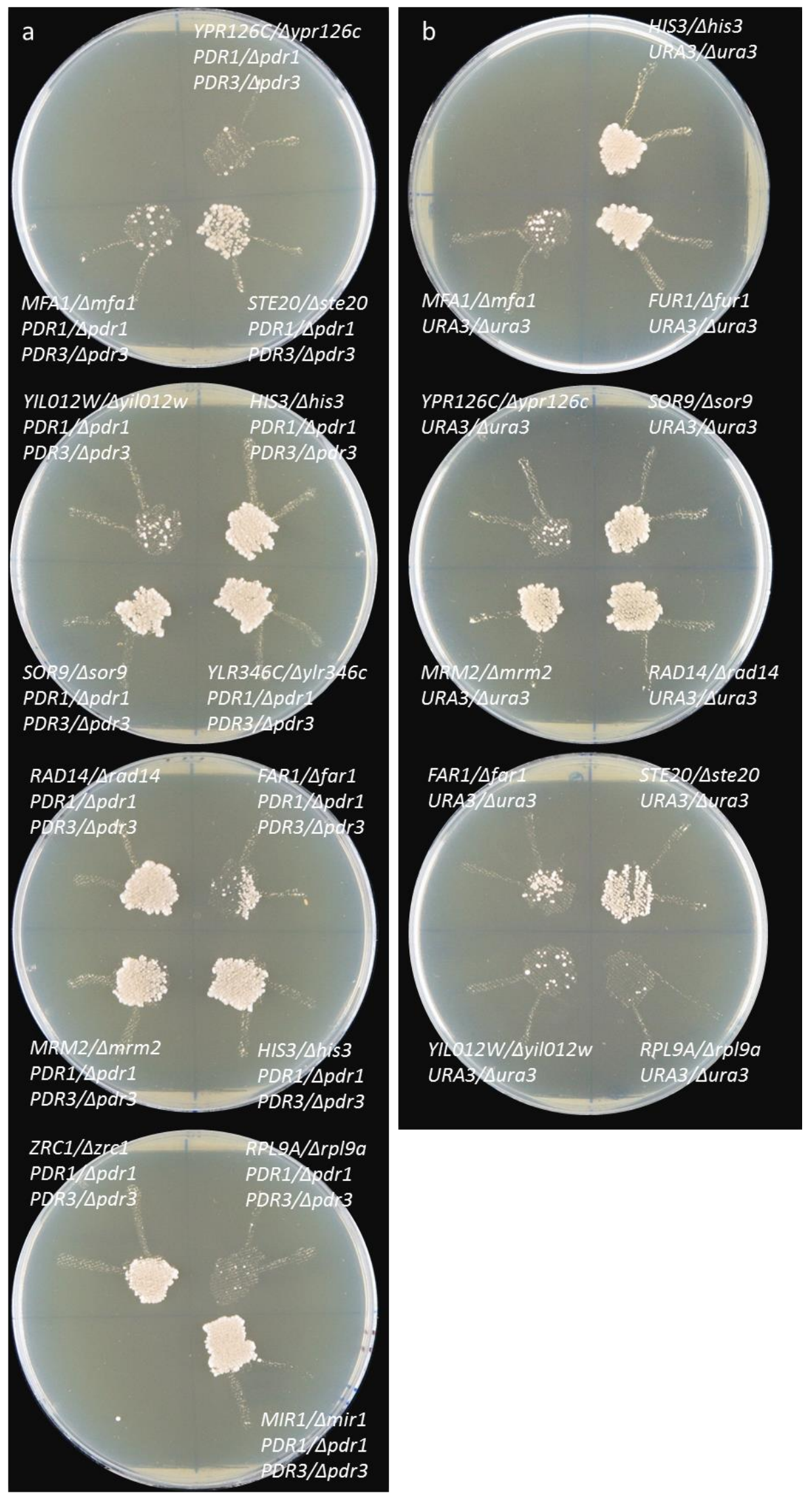
Figure 3.5- PEs resulting from reduced mating efficiency of gene deletion mutants. (a) Crosses involving the PDR-deficient strain. Deletion mutants $\Delta$ mir1, $\Delta m r m 2, \Delta$ rad14, $\Delta s o r 9$, $\Delta y / r 346 c$ and $\Delta z r c 1$ had mating efficiencies comparable of the $\Delta$ his3 control. $\Delta$ far1, $\Delta y i l 012 w$, $\Delta y p r 126 c, \Delta m f a 1, \Delta s t e 20$ and $\Delta r p / 9 a$ mutants had reduced mating efficiencies in comparison to the $\triangle p d r 1 / P D R 1 \Delta p d r 3 / P D R 3 \Delta h i s 3 / \Delta h i s 3$ control. (b) Crosses involving the $\Delta u r a 3$ control strain. Deletion mutants $\Delta s o r 9, \Delta m r m 2, \Delta r a d 14$ and $\Delta f u r 1$ (synthetic lethal control) mated

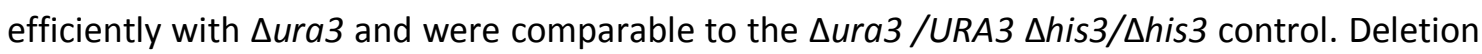
mutants $\Delta$ mfa1, $\Delta$ far1, $\Delta s t e 20, \Delta y i l 012 w, \Delta r p / 9 a$ and $\Delta y p r 126 c$ had reduced mating efficiencies in comparison to the $\triangle$ Ura3 /URA3 $\triangle$ his3/Dhis3 control.

To assess sporulation efficiency, the diploids were sporulated and plated on MATa, MATa $\Delta p d r 1, M A T \mathbf{a} \Delta p d r 3, M A T \mathbf{a} \triangle x x x$ and MATa $\Delta p d r 1 \Delta p d r 3 \Delta x x x$ selection plates. If the strains are sporulation deficient, there will be drastically reduced (i.e. almost none) spores when the MATa haploid selection step of the SGA procedure is applied (middle column of plates on Fig. 3.7). On the other hand if a PE is involved, there will be little difference in the selection plates steps until the final plate where spores with reduced colony size (synthetic sick) or no spores (synthetic lethal) will be observed (right hand plates on Fig. 3.7) because of negative epistasis. Upon sporulation, MATa meiotic progeny (spores) were observed only for the $\Delta h i s 3 \Delta p d r 1 \Delta p d r 3$ mutant control and $\Delta$ mirl $1 \Delta p d r 1 \Delta p d r 3, \Delta r a d 14 \Delta p d r 1 \Delta p d r 3$ and $\Delta y l r 346 c \Delta p d r 1 \Delta p d r 3$ mutants and no synthetic lethal or synthetic sick interactions were observed among these three mutants (Fig. 3.6 \& Table 3.2). Reduced sporulation efficiency was observed for $\Delta m r m 2 \Delta p d r 1 \Delta p d r 3, \Delta r a d 14 \Delta p d r 1 \Delta p d r 3, \Delta s o r 9 \Delta p d r 1 \Delta p d r 3, \quad \Delta y p r 126 c \Delta p d r 1 \Delta p d r 3$, $\Delta y i l 012 w \Delta p d r 1 \Delta p d r 3$ and $\Delta r p l 9 a \Delta p d r 1 \Delta p d r 3$ mutants.

The PDR deletion mutants that showed reduced sporulation efficiency, $\Delta m r m 2$, $\Delta$ rad14, $\Delta$ sor9, $\Delta y p r 126 c, \Delta y i l 012 w$ and $\Delta r p l 9 a$ (also $\Delta m f a 1, \Delta f a r 1$ and $\Delta s t e 20$ ) were mated 


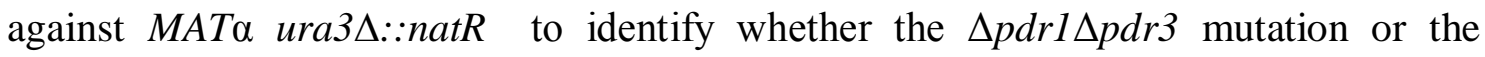
specific $\Delta x x x$ mutation conferred the reduced mating and sporulation efficiency trait. The mating and sporulation efficiencies of the deletion mutants were compared against $\Delta$ furl and $\Delta$ his 3 control crosses. Deletion mutants $\Delta$ sor $9, \Delta m r m 2, \Delta r a d 14$ and $\Delta$ furl had mating efficiencies comparable to the $\Delta$ his 3 control. The crosses involving $\Delta u r a 3$ and gene deletion mutants $\Delta m f a l, \Delta f a r l, \Delta s t e 20, \Delta y i l 012 w, \Delta r p l 9 a$ and $\Delta y p r 126 c$ had reduced mating efficiencies in comparison to the $\Delta$ his 3 control cross (Fig. 3.5b). The deletion mutants that showed reduced mating efficiency with $\Delta p d r l / \Delta p d r 3$ double mutant also showed reduced mating with the $\Delta u r a 3$ control strain showing that it was the individual deletion mutants $(\Delta x x x)$ and not the $\Delta p d r 1 \Delta p d r 3$ mutations that was responsible for the trait. Reduced sporulation efficiencies were observed for crosses involving $\Delta u r a 3$ with all six single gene deletion mutants $\Delta m r m 2, \Delta \operatorname{rad} 14, \Delta s o r 9$, $\Delta y p r 126 c, \Delta y i 1012 \mathrm{w}$ and $\Delta r p l 9 a$ (Fig. 3.7 \& Table. 3.3). These mutants also demonstrated reduced sporulation efficiency with crosses involving $\Delta p d r 1 / \Delta p d r 3$ double mutant. As expected MATa meiotic progeny were observed for $\Delta$ his $3 \Delta u r a 3$ and $\Delta$ furl $\Delta u r a 3$ controls and $\Delta$ furl $\Delta u r a 3$ double mutant was synthetically lethal. Similar colony numbers were expected for $\Delta f u r l$ mutants carrying wild-type copies of $U R A 3$ on the MATa $\Delta x x x$ selection plates ( far right plate on row c Fig. 3.7) as the $\Delta u r a 3$ mutants on MATa $\triangle$ ura3 plates (far right plate on row b on Fig. 3.7). But as s288c background carries $u r a 3 \Delta 0$ mutation there were no wild-type copies of URA3 present, and both ura3 $\Delta 0$ and $u r a 3 \Delta::$ natR mutations were synthetic lethal with $\Delta f u r l$.

It can be concluded that reduced mating and sporulation efficiency was not contributed by the $\Delta p d r 1$ or $\Delta p d r 3$ single mutations or the $\Delta p d r 1 \Delta p d r 3$ double and was a property of these original single deletions in the DMA. 


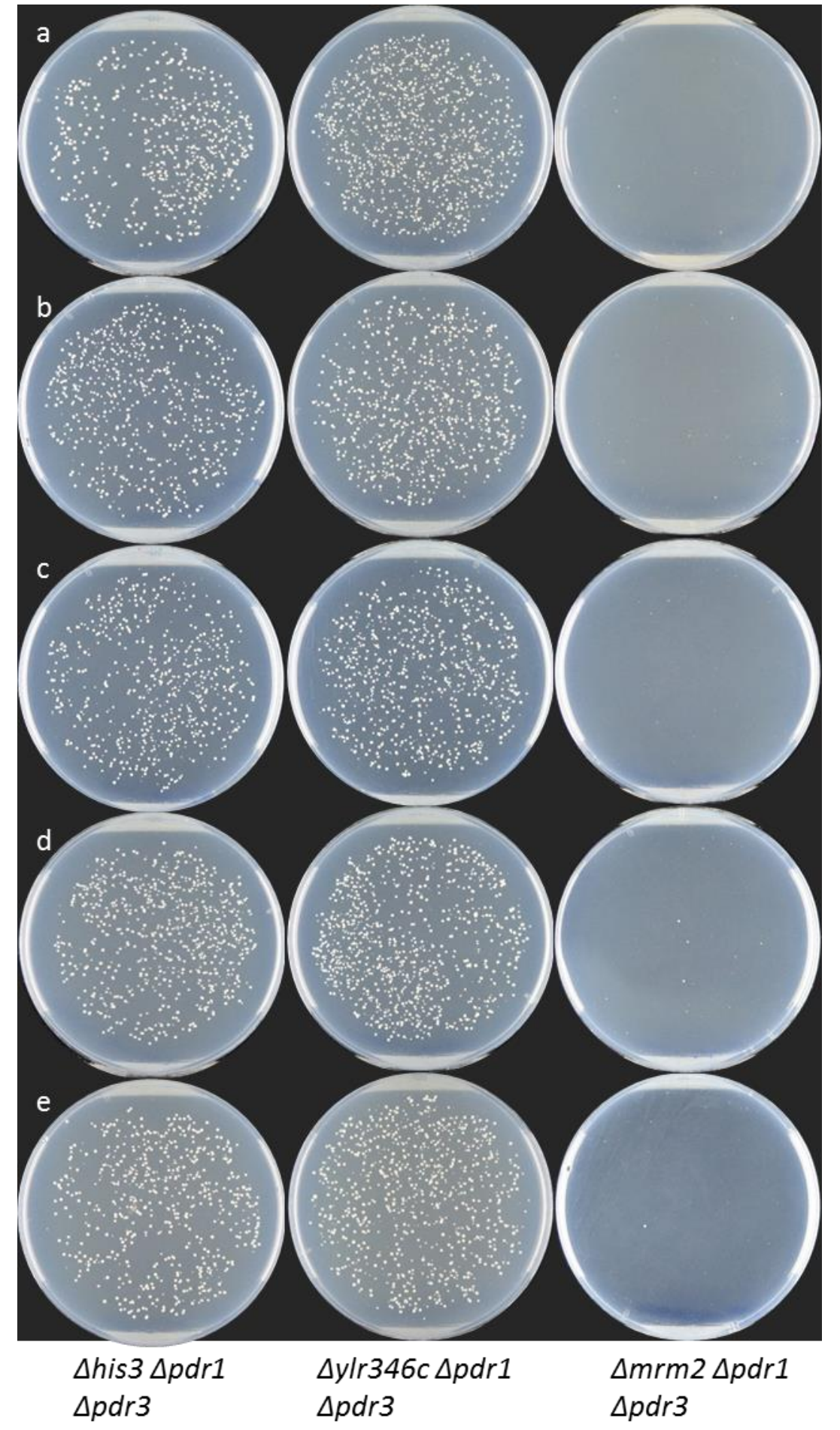

Figure 3.6- Confirmation of putative PEs with PDR-deficient strain. 20, 45, 45, 45 and $180 \mu \mathrm{L}$ volumes of spores /unsporulated diploids were plated on (a) MATa, (b) MATa $\triangle p d r 1$, (c) MATa $\Delta p d r 3,($ d) MATa $\Delta x x x$ and (e) MATa $\Delta p d r 1 \Delta p d r 3 \Delta x x x$ selection plates. The above figure shows the random spore analysis for $\Delta h i s 3 \Delta p d r 1 \Delta p d r 3$ (positive control), $\Delta y / r 346 c \Delta p d r 1 \Delta p d r 3$ (normal sporulation efficiency) and $\Delta m r m 2 \Delta p d r 1 \Delta p d r 3$ (reduced sporulation efficiency) 
mutants. The colony numbers for MATa and MATa $\Delta p d r 1 \Delta p d r 3 \Delta x x x$ selection plates are given in Table 3.2.

\begin{tabular}{|l|l|l|l|l|}
\hline Triple mutant & $\begin{array}{l}\text { Diploid } \\
\text { selection }\end{array}$ & $\begin{array}{l}\text { MATa haploid } \\
\text { selection }\end{array}$ & $\begin{array}{l}\text { MATa Triple } \\
\text { mutants }\end{array}$ & $\begin{array}{l}\text { SL or SS } \\
\text { interactions }\end{array}$ \\
\hline$\Delta$ his3 $\Delta p d r 1 \Delta p d r 3$ & normal & 474 & 503 & No \\
\hline$\Delta m i r 1 \Delta p d r 1 \Delta p d r 3$ & normal & 465 & 501 & No \\
\hline$\Delta m r m 2 \Delta p d r 1 \Delta p d r 3$ & normal & 0 & - & - \\
\hline$\Delta r a d 14 \Delta p d r 1 \Delta p d r 3$ & normal & 2 & - & - \\
\hline$\Delta$ sor9 $\Delta p d r 1 \Delta p d r 3$ & normal & 1 & - & - \\
\hline$\Delta y / r 346 c \Delta p d r 1 \Delta p d r 3$ & normal & 412 & 387 & No \\
\hline$\Delta z r c 1 \Delta p d r 1 \Delta p d r 3$ & normal & 361 & 499 & No \\
\hline$\Delta r p / 9 a \Delta p d r 1 \Delta p d r 3$ & reduced & 0 & - & - \\
\hline$\Delta y i l 012 w \Delta p d r 1 \Delta p d r 3$ & reduced & 2 & - & - \\
\hline$\Delta y p r 126 c \Delta p d r 1 \Delta p d r 3$ & reduced & 1 & - & - \\
\hline
\end{tabular}

Table 3.2- Confirmation of putative PEs with PDR-deficient strain. The sporulation efficiency (colony numbers) of $\Delta m i r 1, \Delta z r c 1$ and $\Delta y / r 346 c$ mutants were comparable to the $\Delta h i s 3$ control and no synthetic lethal (SL) or synthetic sick (SS) interactions were observed for $\Delta$ mir1 $\Delta p d r 1 \Delta p d r 3, \Delta z r c 1 \Delta p d r 1 \Delta p d r 3$ and $\Delta y / r 346 c \Delta p d r 1 \Delta p d r 3$ triple mutants. $\Delta m r m 2, \Delta r a d 14$, $\Delta$ sor9, $\Delta y p r 126 c, \Delta y i l 012 \mathrm{w}$ and $\Delta r p / 9 a$ deletion mutants had reduced sporulation efficiencies in comparison to the $\Delta h i s 3$ control. 


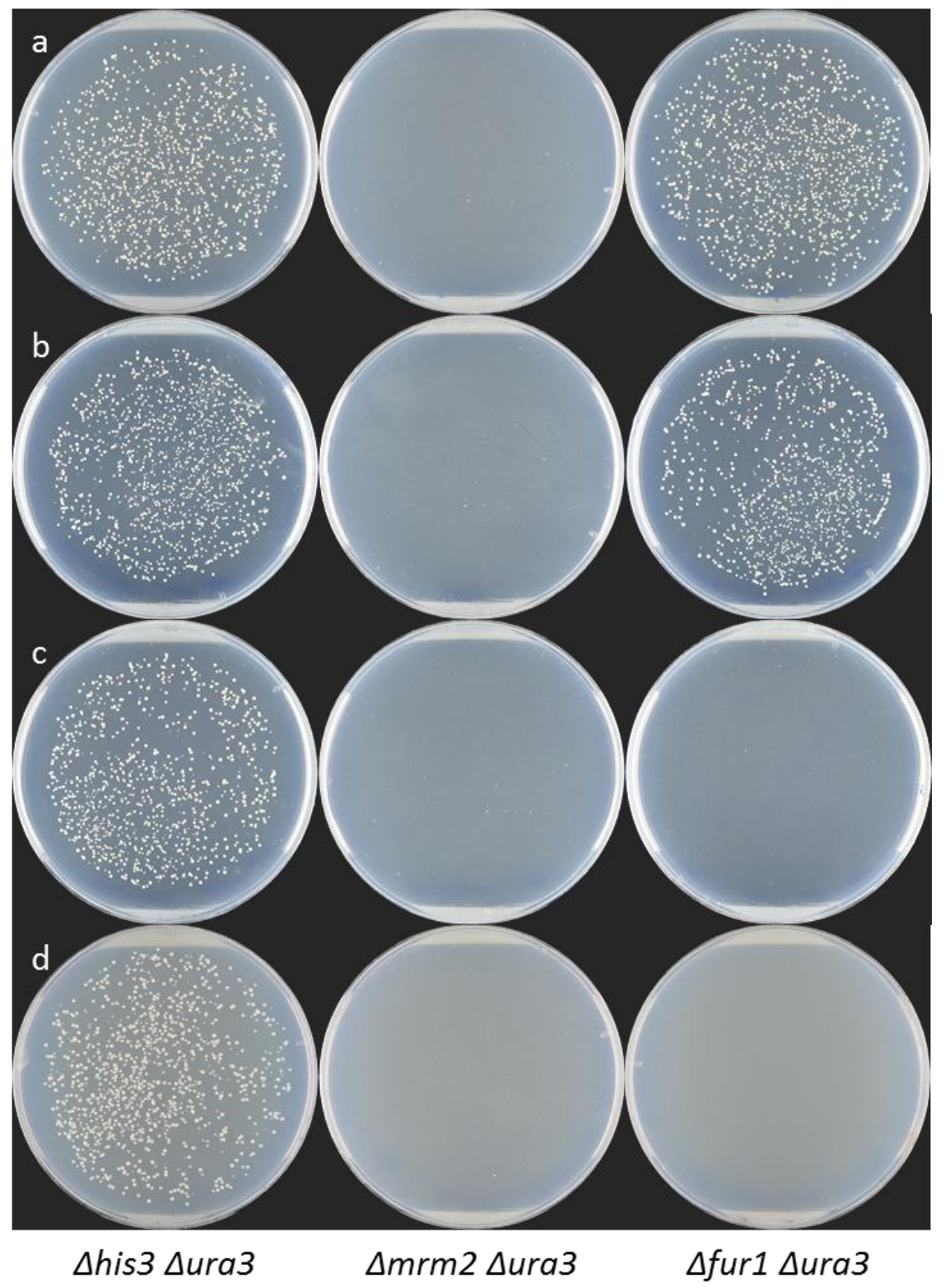

Figure 3.7- PEs resulting from reduced sporulation efficiency of gene deletion mutants. 15, 30, 30 and $60 \mu \mathrm{L}$ volumes of spores/unsporulated diploids were plated on (a) MATa, (b) MATa $\triangle u r a 3$, (c) MATa $\triangle x x x$ and (d) MATa $\triangle u r a 3 \Delta x x x$ selection plates. The figure above shows the random spore analysis for $\Delta$ his $3 \Delta u r a 3$ (positive control), $\Delta m r m 2 \Delta u r a 3$ (reduced sporulation efficiency), and $\Delta$ fur1 $1 \Delta u r a 3$ (SL control). Colonies of similar size and numbers were observed for the $\Delta$ his $3 \Delta u r a 3$ control across all plates. Reduced number of colonies was observed for $\Delta m r m 2 \Delta u r a 3$ across all selection plates. A similar number of colonies were observed for the

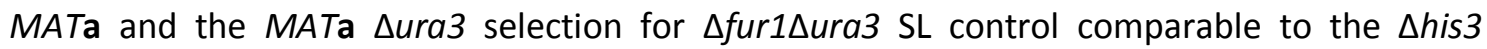
control and a significantly reduced number of colonies were observed for MATa $\triangle$ fur1 or MATa $\Delta$ Ura3 $\Delta f u r 1$ selection plates which was indicative of a synthetic lethal interaction. The colony numbers for MATa and MATa $\triangle$ ura3 $\Delta x x$ selection plates are given in Table 3.3. 


\begin{tabular}{|c|c|c|c|c|}
\hline Double mutant & $\begin{array}{l}\text { Diploid selection } \\
\text { efficiency }\end{array}$ & $\begin{array}{l}\text { MATa haploid } \\
\text { selection }\end{array}$ & $\begin{array}{l}\text { MATa double } \\
\text { mutants }\end{array}$ & $\begin{array}{l}\text { SL or SS } \\
\text { interactions }\end{array}$ \\
\hline 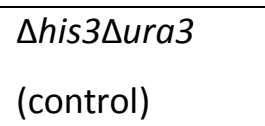 & Normal & 302 & 379 & No \\
\hline$\Delta m r m 2 \Delta u r a 3$ & Normal & 0 & - & - \\
\hline 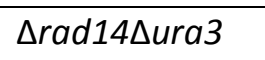 & Normal & 1 & - & - \\
\hline 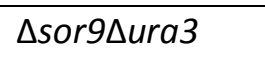 & Normal & 2 & - & - \\
\hline 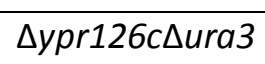 & Reduced & 1 & - & - \\
\hline$\Delta$ far1 $\Delta$ ura3 & Reduced & 1 & - & - \\
\hline 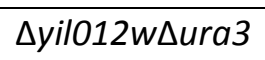 & Reduced & 0 & - & - \\
\hline 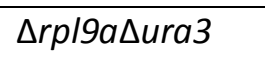 & Reduced & 0 & - & - \\
\hline $\begin{array}{l}\Delta \text { fur1sura3 } \\
\text { (SL control) }\end{array}$ & Normal & 390 & 1 & $\mathrm{SL}$ \\
\hline
\end{tabular}

Table 3.3 - PEs resulting from reduced sporulation efficiency of gene deletion mutants. Only the $\Delta$ his3 (control) and $\Delta f u r 1$ (SL control) sporulated efficiently (colony numbers) in crosses involving $\Delta u r a 3$, and $\Delta$ fur1sura3 was synthetically lethal. $\Delta m r m 2, \Delta \operatorname{rad} 14, \Delta s o r 9, \Delta y p r 126 c$, $\Delta f a r 1, \Delta y i l 012 w$ and $\Delta r p / 9 a$ had reduced sporulation efficiencies in comparison to the $\Delta h i s 3$ control for crosses involving $\Delta$ ura3 explaining their little or no-growth appearance in the SGA plates.

3.3 Loss of Pdr1p and Pdr3p transcription factors does not impair or induce the $\underline{\text { unfolded protein response (UPR) }}$

PDR-deficient strain, $\triangle p d r 1 \Delta p d r 3 \quad U P R E-G F P$ and wild-type strain, UPRE-GFP (Chapter 2 Materials and Methods) were treated with UPR inducing compounds dithiothreitol (DTT) and tunicamycin. UPR induction was assayed by measuring GFP expression driven from the UPR element (UPRE) promoter using confocal microscopy (Fig. 3.8a-c). 
For the wild-type strain, UPRE-GFP, UPRE expression was low under untreated conditions and as expected increased in a dose dependent manner in the presence of DTT and tunicamycin as expected. Similar results were also observed for the PDRdeficient strain, $\triangle p d r 1 \Delta p d r 3$ UPRE-GFP where UPRE expression was low under untreated conditions and it increased steadily with increase in concentration of DTT and tunicamycin. The UPRE levels were not significantly different between the PDRdeficient strain and wild-type strain in the absence of DTT and tunicamycin. Upon UPRE induction with DTT and tunicamycin UPRE levels were again not significantly different for the PDR-deficient strain and the wild-type strain. Thus, it can be concluded that the loss of Pdr1p and Pdr3p transcription factors does not induce or impair the UPR response.

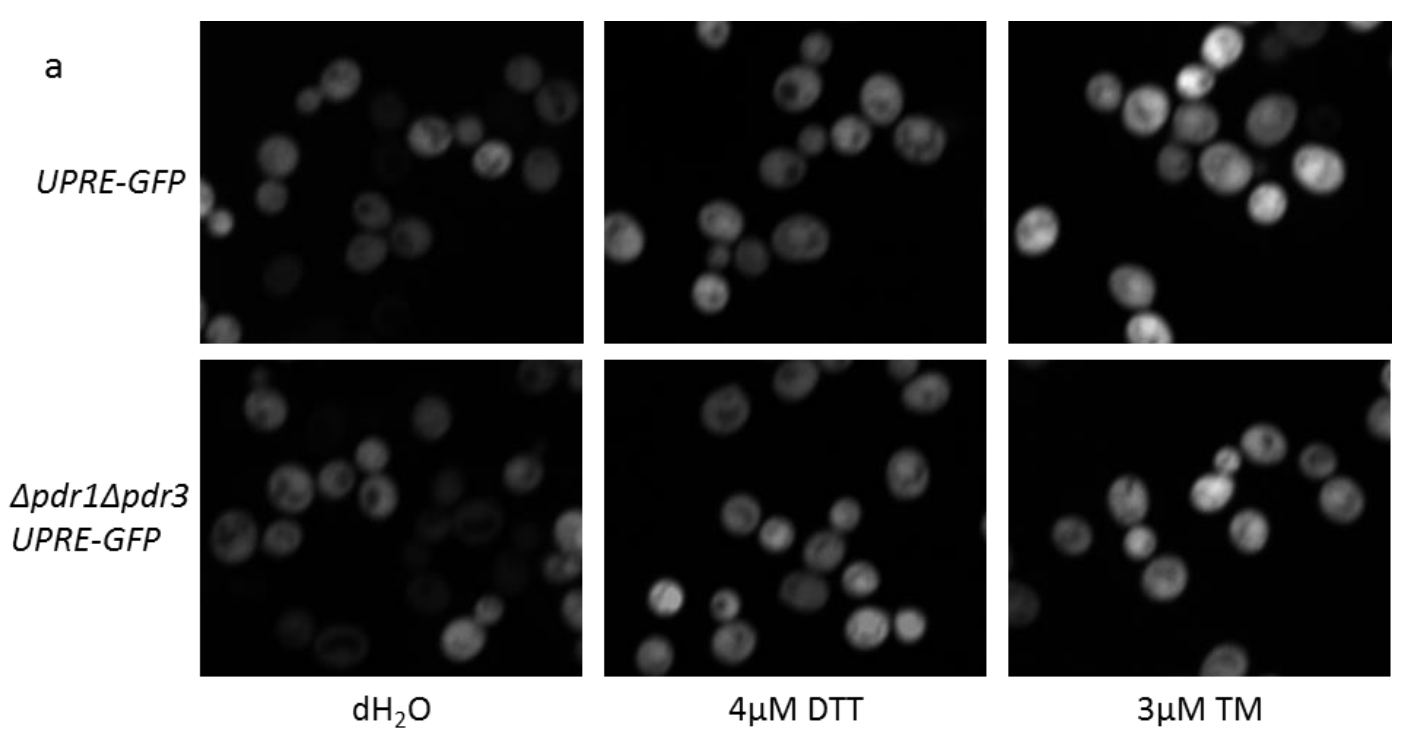




\begin{tabular}{|l|l|l|l|l|l|l|l|l|}
\hline DTT [ $\mu \mathrm{M}]$ & $\mathrm{dH}_{\mathbf{2}} \mathbf{0}$ & $\mathbf{0 . 0 6}$ & $\mathbf{0 . 1 3}$ & $\mathbf{0 . 2 5}$ & $\mathbf{0 . 5}$ & $\mathbf{1}$ & $\mathbf{2}$ & $\mathbf{4}$ \\
\hline $\begin{array}{l}\Delta p d r 1 \Delta p d r 3 \\
\text { UPRE-GFP (RFU) }\end{array}$ & 496.83 & 565.3 & 522.16 & 607.37 & 809.27 & $\begin{array}{l}1283.1 \\
9\end{array}$ & 1725.11 & 1725.65 \\
\hline $\begin{array}{l}\text { UPRE-GFP } \\
\text { (RFU) }\end{array}$ & 440.69 & 484.42 & 499.96 & 561.02 & 852.69 & $\begin{array}{l}1517.7 \\
9\end{array}$ & 1947.67 & 1811.01 \\
\hline
\end{tabular}

c

\begin{tabular}{|l|l|l|l|l|l|l|l|l|}
\hline $\mathrm{TM}[\mu \mathrm{M}]$ & $\mathrm{dH}_{\mathbf{2}} \mathbf{0}$ & $\mathbf{0 . 0 5}$ & $\mathbf{0 . 0 9}$ & $\mathbf{0 . 1 9}$ & $\mathbf{0 . 3 8}$ & $\mathbf{0 . 7 5}$ & $\mathbf{1 . 5}$ & $\mathbf{3}$ \\
\hline $\begin{array}{l}\Delta p d r 1 \Delta p d r 3 U P R E \\
- \text { GFP (RFU) }\end{array}$ & 694.26 & 655.26 & 611.81 & 545.5 & 658.18 & 627.04 & 1049.62 & 1429 \\
\hline $\begin{array}{l}\text { UPRE-GFP } \\
\text { (RFU) }\end{array}$ & 542.6 & 504.93 & 541.18 & 480.52 & 484.7 & 618.51 & 978.54 & 1292.3 \\
\hline
\end{tabular}

Figure 3.8 - Loss of Pdr1p and Pdr3p transcription factors does not induce nor impair unfolded protein response (UPR). The images were cropped using Image J (rsbweb.nih.gov/ij/). The GFP intensity for each cell in each well was measured using the OPERA confocal microscope and Acapella and averaged GFP intensities for duplicate experiments are given in the table above. (a) UPRE induction of PDR-deficient strain, $\triangle p d r 1 \triangle p d r 3$ UPRE-GFP and wild-type strain, UPRE-GFP with DTT and tunicamycin (TM). (b) The UPRE induction levels were lowest under untreated conditions for both wild-type and PDRdeficient strains and it steadily increased with increasing concentrations of DTT. The PDRdeficient strain, $\triangle p d r 1 \Delta p d r 3$ UPRE-GFP had UPRE induction levels comparable to and wild-type strain, UPRE-GFP across all concentrations of DTT tested. (c)The UPRE induction in response to tunicamycin treatment was not observed until $1.5 \mu \mathrm{M}$ tunicamycin. The UPRE induction levels across all concentrations of tunicamycin were similar for the PDR-deficient strain and wild-type strain. 
3.4 Loss Pdr1p and Pdr3p transcription factors does not induce nor impair oxidative $\underline{\text { stress response regulator YAP1 }}$

The wild-type strain, YAP1-GFP and the PDR-deficient strain, $\triangle p d r 1 \triangle p d r 3$ YAP1-GFP were treated with compounds associated with reactive oxygen species (ROS) generation; menadione and $\mathrm{H}_{2} \mathrm{O}_{2}$, in addition the xenobiotics; latrunculin-A, a Pdr5p substrate (Chapter 4-Fig. 4.2) and rapamycin, which is not PDR substrate. Activation of Yap1p was detected by nuclear localisation of GFP using confocal microscopy (Fig. 3.9a-b).

Yap1p had a cytoplasmic location under untreated conditions for both the wild-type and the PDR-deficient strains. Rapid nuclear localisation of Yap1p was observed for both the wild-type and the PDR-deficient strain following treatment with menadione and $\mathrm{H}_{2} \mathrm{O}_{2}$ showing a functional oxidative stress response for both the wild-type and the PDR-deficient strain. Interestingly, nuclear localisation of Yap1p was not observed for either wild type or the PDR-deficient strain following treatment with the xenobiotics latrunculin-A or rapamycin. The oxidative stress response was not induced under xenobiotic treated conditions including PDR substrates even in the absence of Pdr1p and Pdr3p transcription factors. This suggests that Yap1p plays a role in oxidative stress response but not in mediating the PDR phenotype. In the above experiment different concentrations of latrunculin-A was used for YAP1-GFP and the $\triangle p d r 1 \Delta p d r 3 Y A P 1$ GFP strain due to increased sensitivity of the PDR-deficient strain to PDR substrates. 
a

YAP1-GFP

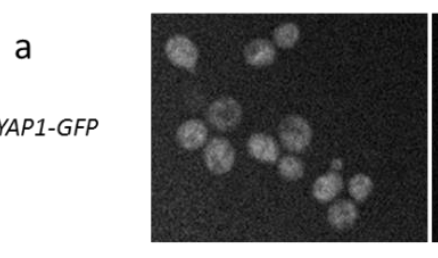

$\mathrm{dH} 2 \mathrm{O}$

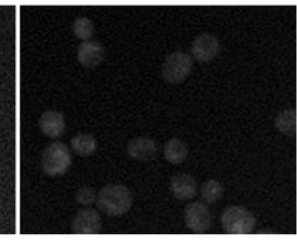

30 nM Rapamycin

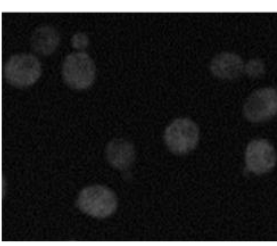

$8 \mu \mathrm{M}$ Latrunculin A

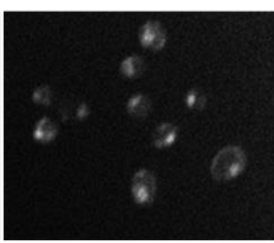

$100 \mu \mathrm{M}$ Mennadione

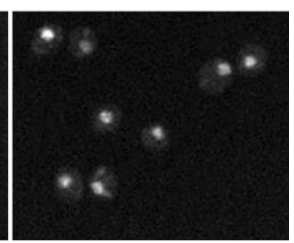

$1 \mathrm{mM} \mathrm{H}_{2} \mathrm{O}_{2}$

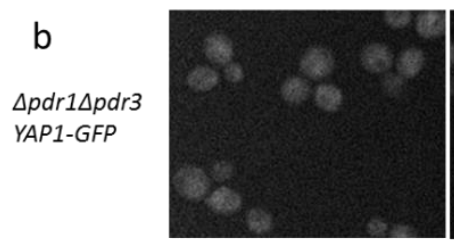

$\mathrm{dH} 2 \mathrm{O}$

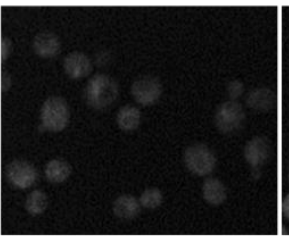

30 nM Rapamycin

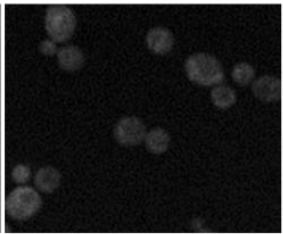

$3 \mu \mathrm{M}$ Latrunculin A

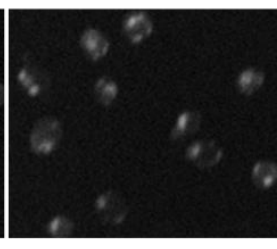

$100 \mu \mathrm{M}$ Mennadione

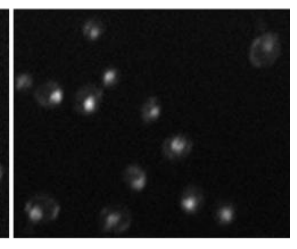

$1 \mathrm{mM} \mathrm{H}_{2} \mathrm{O}_{2}$

Figure 3.9 - Loss of Pdr1p and Pdr3p transcription factors does not induce or impair oxidative stress response regulator Yap1p. The wild-type strain, YAP1-GFP, and the PDR-deficient strain, $\triangle p d r 1 \triangle p d r 3 Y A P 1-G F P$ was treated with xenobiotics latrunculin-A (a PDR substrate) and rapamycin (not a PDR substrate) and ROS generating compounds menadione and $\mathrm{H}_{2} \mathrm{O}_{2}$ at MIC. The images were cropped using Image J (rsbweb.nih.gov/ij/).(a) Nuclear localisation of Yap1p was observed for the wild-type strain, YAP1-GFP, in response to treatment with menadione and $\mathrm{H}_{2} \mathrm{O}_{2}$ but not with latrunculin-A, rapamycin or under untreated conditions. (b) Nuclear localisation of Yap1p was observed for the PDR-deficient strain, $\triangle p d r 1 \Delta p d r 3 Y A P 1-G F P$, in response to treatment with menadione and $\mathrm{H}_{2} \mathrm{O}_{2}$ but not with latrunculin-A or rapamycin or under untreated conditions.

\subsection{Genome-wide sensitisation of the deletion set to PDR substrates}

Pools of PD-DMA ( $\Delta p d r 1 \Delta p d r 3 \Delta x x x)$ and wild-type background DMA $(\Delta x x x)$ were used in a dose response assay employing cycloheximide, which is a PDR substrate and rapamycin, which is not. The growth was determined by measuring optical density of the culture after incubation in the presence of the compound or the carrier solvent. The two deletion pools differed in their sensitivity to PDR substrate cycloheximide (Fig. 3.10a). The DMA was more resistant to cycloheximide and had an $\mathrm{IC}_{50}$ of $53.80 \mathrm{nM}$. The PD-DMA was the less resistant to cycloheximide with an $\mathrm{IC}_{50}$ of $163.47 \mathrm{nM}$. The 
PD-DMA demonstrated a $\sim 3$ fold increase in sensitivity to cycloheximide in comparison to DMA. The two deletion pools had sensitivities that were not significantly different for rapamycin (Fig. 3.10b). The DMA and PD-DMA had rapamycin $\mathrm{IC}_{50}$ of $79.81 \mathrm{nM}$ and $85.62 \mathrm{nM}$, respectively.

These results were consistent previous findings for the PDR-deficient strain, $\Delta p d r 1 \Delta p d r 3$, and the wild-type strain. The two strains demonstrated similar sensitivities to rapamycin which is not PDR substrate and differed in their sensitivities to PDR substrate cycloheximide with PDR-deficient strain showing less resistance to cycloheximide than the wild-type strain.
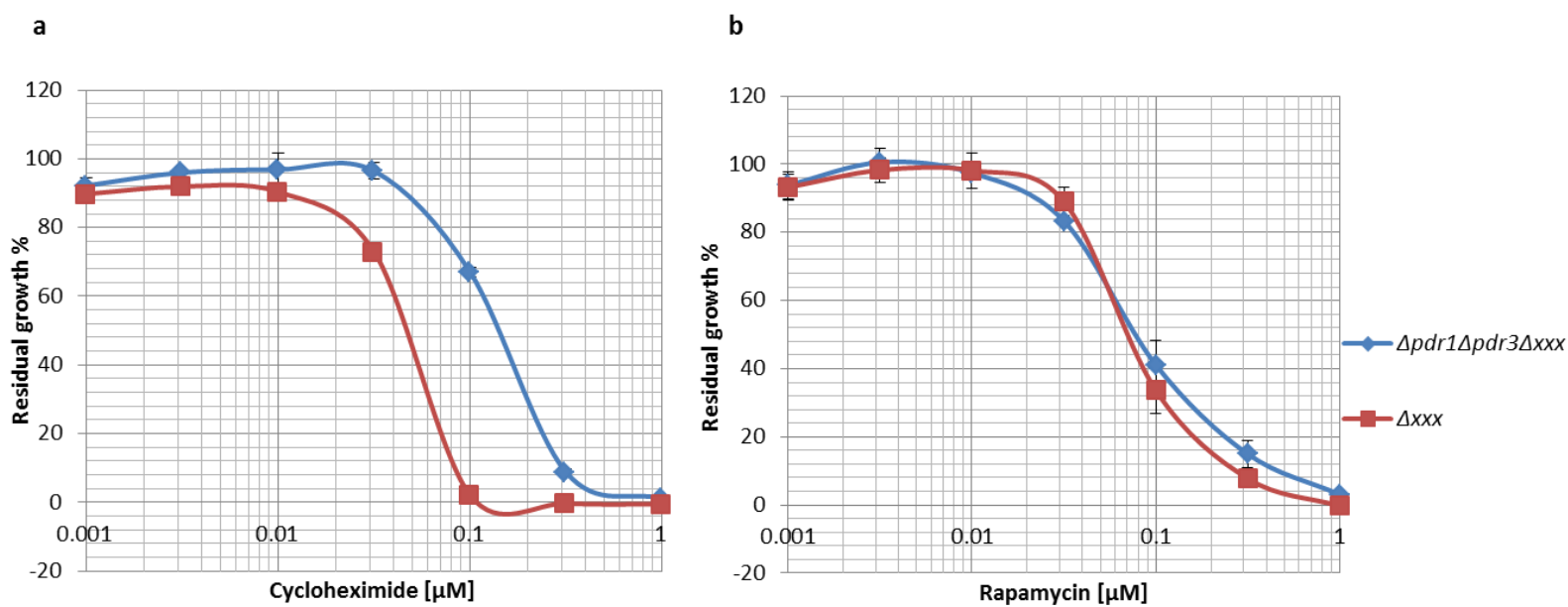

Figure 3.10- Differential sensitivity of the deletion mutants pools to PDR substrate cycloheximide. In a dose response assay PD-DMA $(\Delta p d r 1 \Delta p d r 3 \Delta x x x)$ and the DMA $(\Delta x x x)$ pools were assessed in their sensitivity to cycloheximide, which is a PDR substrate and rapamycin, which is not. (a) The PD-DMA was the most sensitive and had a $\sim 3$ fold increase in sensitivity to cycloheximide in comparison to DMA in wild-type background. (b) The PD-DMA and the DMA had similar sensitivities to rapamycin. 


\subsection{Deletion mutants hypersensitive to rapamycin}

The sensitivities of the deletion mutants to rapamycin were assessed by pinning the PDDMA onto SC agar plates containing $5 \mathrm{nM}$ rapamycin $\left(\sim \mathrm{IC}_{30}\right)$. Rapamycin is an inhibitor of TORC1, a Ser/Thr protein kinase which plays major role in cell metabolism and growth (74). Rapamycin in yeast forms a complex with the protein complex Frp1p which consequently binds TORC1 and inhibits its function causing growth arrest (74).

The relative growth rates for each strain was assessed by comparing colony size on agar plates utilising colony HT and SESA and were compared against mutants treated with the carrier solvent DMSO. Three independent pinning array experiments identified 18 phenotypic enhancements with rapamycin (Table 3.4). These were compiled in to a network diagram using Cytoscape (Chapter 2 Materials and Methods) based on statistical overrepresentation of GO categories. The deletion mutants hypersensitive to rapamycin included TOR1, $P H O 80$ and genes responsible for macromolecule localisation (SSD1, VPS53, GTR1, VPS27, VPS51, PEP8, and GTR2), lipid metabolism genes (OPI3 and ERG2) and several other genes (CKA2, ESO1, TRR2, EMC8, PKR1, YELO57C and YDL121C). The genes TOR1 and PHOSO were enriched for the GO category "negative regulation of autophagy" ( 79.5 fold enrichment) and genes VPS51, PEP8 and VPS53 for "retrograde transport from endosome to Golgi" ( 19.9 fold enrichment) (Fig. 3.11).

These were as expected, as TOR 1 with the essential gene TOR 2 encodes for the target of rapamycin TORC1. TORC1 negatively regulates various catabolic processes: 
retrograde transport and macroautophagy, a process which hydrolyses surplus cytoplasmic content. Both of these pathways generate nutrients for cellular adaptation under nutrient starvation or TORC1 inhibited conditions. The downregulation of TORC1 pathway by loss of TOR1 or loss of genes that are expressed under TORC1 inhibition disables the adaptive response needed to overcome rapamycin induced growth arrest.

\begin{tabular}{|l|l|l|}
\hline ORF & Gene & Description \\
\hline YOR061W & CKA2 & Alpha' catalytic subunit of casein kinase 2 (CK2) \\
\hline YBR076W & ECM8 & Non-essential protein of unknown function \\
\hline YNL080C & EOS1 & Protein involved in N-glycosylation \\
\hline YMR202W & ERG2 & C-8 sterol isomerase \\
\hline YML121W & GTR1 & $\begin{array}{l}\text { GTP binding protein and negative regulator of the Ran/Tc4 GTPase } \\
\text { cycle }\end{array}$ \\
\hline YGR163W & GTR2 & Putative GTP binding protein that regulates the Ran/Tc4 GTPase cycle \\
\hline YJR073C & OPI3 & Phospholipid methyltransferase \\
\hline YJL053W & PEP8 & Sorting protein that is a subunit of the retromer complex \\
\hline YOL001W & PHO80 & Cyclin \\
\hline YMR123W & PKR1 & V-ATPase assembly factor \\
\hline YDR293C & SSD1 & Translational repressor with a role in polar growth and wall integrity \\
\hline YJR066W & TOR1 & PIK-related protein kinase and rapamycin target \\
\hline YHR106W & TRR2 & Mitochondrial thioredoxin reductase \\
\hline YNR006W & VPS27 & Endosomal protein that forms a complex with Hse1p \\
\hline YKR020W & VPS51 & Component of the Golgi-associated retrograde protein complex \\
\hline YJL029C & VPS53 & Component of the Golgi-associated retrograde protein complex \\
\hline YDL121C & YDL121C & Putative protein of unknown function \\
\hline YEL057C & YEL057C & Protein of unknown function involved in telomere maintenance \\
\hline
\end{tabular}


Table 3.4 - List of gene deletion mutants hypersensitive to $5 \mathrm{nM}$ rapamycin. 18 phenotypic enhancements were identified for rapamycin against the PD-DMA from 3 independent pinning experiments employing colony HT and SESA. The genes were annotated according to GO function as stated in SGD project http://www.yeastgenome.org/download-data/ (01-07-2012) (75). Highlited in blue are genes involved in the TOR pathway.

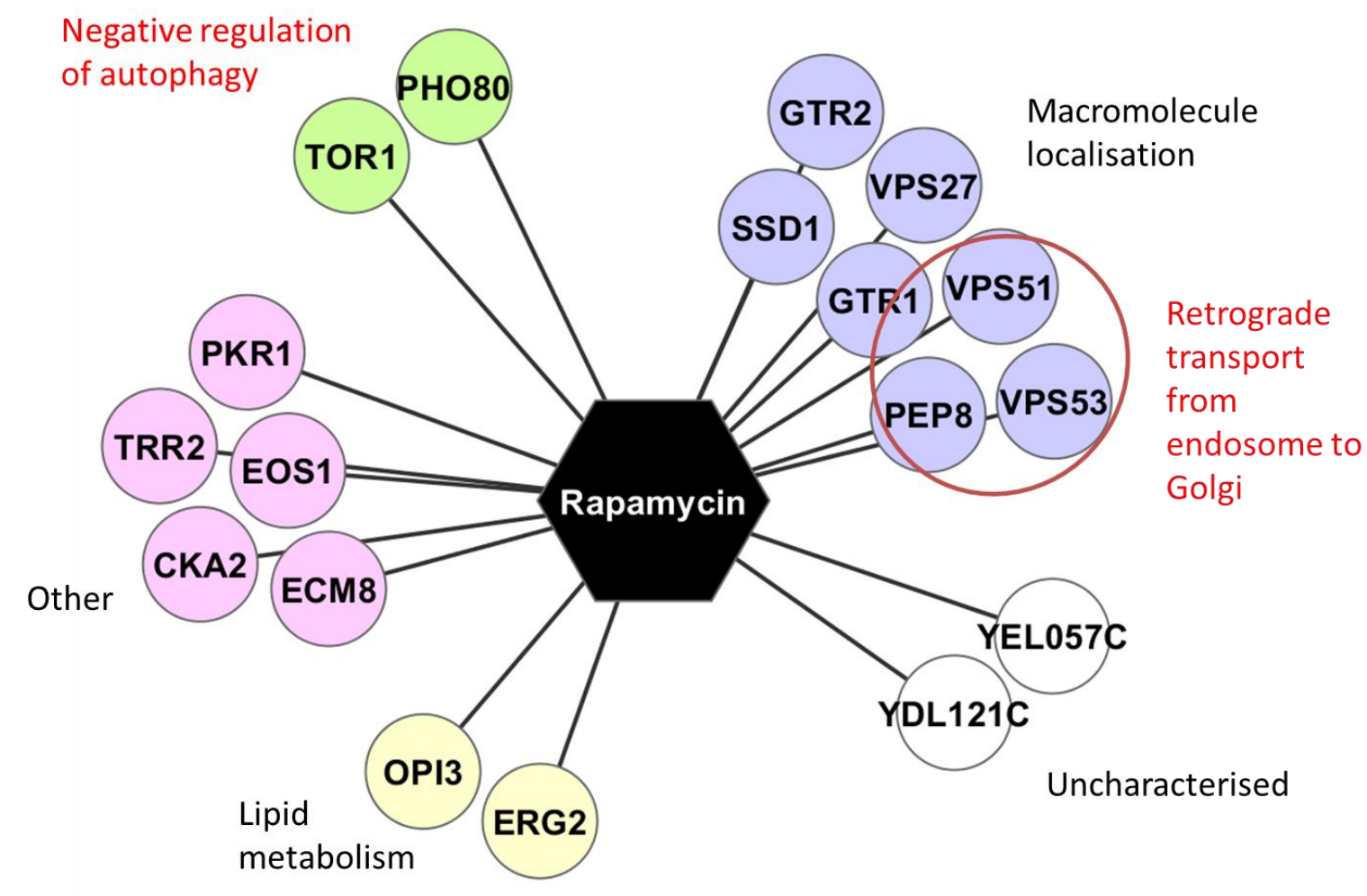

Figure 3.11 - Network diagram of genes hypersensitive to $5 \mathrm{nM}$ rapamycin. Genes were compiled in to network diagram using Cytoscape and assigned to GO terms by methodology based on statistical representation. Genes were enriched for negative regulation of autophagy and retrograde transport from endosome to Golgi. 


\subsection{Deletion mutants hypersensitive to cycloheximide}

The sensitivities of the haploid PDR-deficient strains to cycloheximide were assessed as a pool by DNA barcode microarrays (Chapter 2 Materials and Methods) that measures relative fitness of strains in the pool under drug treatment. Cycloheximide is an inhibitor of eukaryotic translation (76).

The competitive hybridisation ( $\mathrm{Cy} 5 / \mathrm{Cy} 3$ ratios) of barcodes denoting different strains in cycloheximide treated and untreated conditions to oligos on the microarray chip was used to measure the competitive fitness of each PDR-deficient strains. The barcode microarray experiment identified 31 phenotypic enhancements with cycloheximide (Table 3.5). These included genes encoding the large (60s) subunit of ribosome (RPL9A, RPL36A, RPL13A, RPL4A, RPP1B, RPL37A and RPL23A), genes involved in translational elongation (EFT2, RPP1B and GCN20), transcription (MBF1 and SOH1) cytoskeletal organisation (CAP1 and SWI4) and multi-drug resistance (Fig. 3.11). The genes RPL9A, RPL36A, RPL13A, RPL4A, RPP1B, RPL37A and RPL23A were enriched for GO category, "cytoplasmic translation" ( 6.7 fold enrichment) and RPL9A, RPL36A, RPL13A, RPLAA, RPP1B, EFT2, RPL37A and RPL23A enriched for the GO category "translation" ( $\sim 5.1$ fold enrichment).

Such hypersensitive mutants were as expected, because genes enriched cytoplasmic translation encodes components for large (60S) subunit of the ribosome and along with translational elongation genes which mediate the translation. The increased sensitivity 
of several transcription and cytoskeletal organisation genes to the eukaryotic translation inhibitor cycloheximide suggest that they may play a role in translation.

\begin{tabular}{|c|c|c|}
\hline ORF & Gene & Description \\
\hline YKL007W & CAP1 & Alpha subunit of the capping protein (CP) heterodimer \\
\hline YJR048W & CYC1 & Cytochrome c \\
\hline YBR078W & ECM33 & GPI-anchored protein of unknown function \\
\hline YDR385W & EFT2 & Elongation factor 2 (EF-2) \\
\hline YNL080C & EOS1 & Protein involved in N-glycosylation \\
\hline YMR202W & ERG2 & C-8 sterol isomerase \\
\hline YLR377C & FBP1 & Fructose-1,6-bisphosphatase \\
\hline YFR009W & GCN20 & Positive regulator of the Gcn2p kinase activity \\
\hline YKL017C & HCS1 & Hexameric DNA polymerase alpha-associated DNA helicase A \\
\hline YMR132C & JLP2 & Protein of unknown function \\
\hline YNL323W & LEM3 & Membrane protein of the plasma membrane and ER \\
\hline $\begin{array}{l}\text { YOR298C- } \\
\text { A }\end{array}$ & MBF1 & Transcriptional coactivator \\
\hline YOR231W & MKK1 & MAPKK involved in the protein kinase $\mathrm{C}$ signaling pathway \\
\hline YDL082W & RPL13A & Protein component of the large (60S) ribosomal subunit \\
\hline YBL087C & RPL23A & Protein component of the large (60S) ribosomal subunit \\
\hline YMR194W & RPL36A & $\mathrm{N}$-terminally acetylated protein component of the $60 \mathrm{~S}$ ribosomal subunit \\
\hline YLR185W & RPL37A & Protein component of the large (60S) ribosomal subunit \\
\hline YBR031W & RPL4A & $\mathrm{N}$-terminally acetylated protein component of the $60 \mathrm{~S}$ ribosomal subunit \\
\hline YGL147C & RPL9A & Protein component of the large (60S) ribosomal subunit \\
\hline YDL130W & RPP1B & Ribosomal protein P1 beta \\
\hline YML013W & UBX2 & Bridging factor involved in ER-associated protein degradation \\
\hline YNL032W & SIW14 & Tyrosine phosphatase involved in actin organization and endocytosis \\
\hline YPR189W & SKI3 & Ski complex component and TPR protein \\
\hline YGL127C & $\mathrm{SOH} 1$ & Subunit of the RNA polymerase II mediator complex \\
\hline YPL157W & TGS1 & Trimethyl guanosine synthase \\
\hline YDR049W & VMS1 & Component of a Cdc48p-complex involved in protein quality control \\
\hline
\end{tabular}




\begin{tabular}{|l|l|l|}
\hline YGL211W & NCS6 & Protein required for uridine thiolation at the wobble position of Gln \\
\hline YGL082W & YGL082W & Putative protein of unknown function \\
\hline YIR042C & YIR042C & Putative protein of unknown function \\
\hline YMR247C & RKR1 & RING domain E3 ubiquitin ligase \\
\hline $\begin{array}{l}\text { YMR316C- } \\
\text { A }\end{array}$ & YMR316C- & Protein of unknown function \\
\hline
\end{tabular}

Table 3.5 - List of gene deletion mutants hypersensitive to $30 \mathrm{nM}$ cycloheximide. 31 phenotypic enhancements were identified from a DNA barcode microarray utilizing the haploid PDR-deficient mutants. Genes were annotated as described in SGD project http://www.yeastgenome.org/download-data/ (01-07-2012) (75). Highlighted in blue are genes encoding components of the large (60s) subunit of the ribosome and translation elongation genes. 


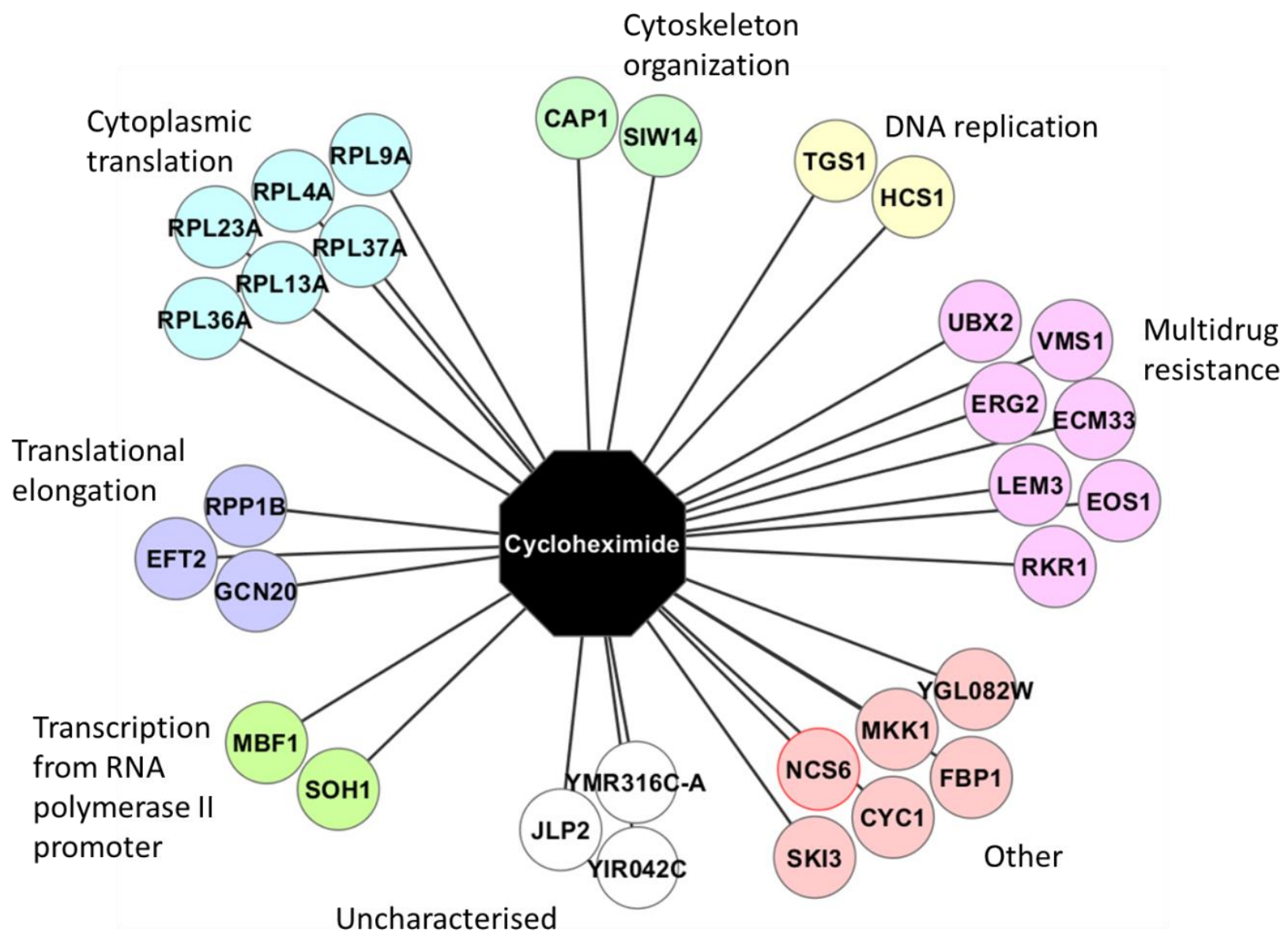

Figure 3.12 - Network diagram of genes hypersensitive to $30 \mathrm{nM}$ cycloheximide. Genes were compiled in to the network diagram using Cytoscape and assigned to GO terms by methodology based on statistical over representation. Genes enriched for biological processes cytoplasmic translation and translation.

\subsubsection{The PDR-deficient mutants identify more bioactives from the LOPAC library.}

Pools of DMA $(\Delta x x x)$ and PDR deficient-DMA ( $\Delta p d r 1 \Delta p d r 3 \Delta x x x)$ strains were screened against the LOPAC library comprising 1280 pharmacologically active compounds in a growth inhibitory assay by measuring OD. The DMA in wild-type background identified five bioactive compounds from the LOPAC library and the PDR deficient-DMA identified the same five compounds and another 20 additional 
compounds as having biological activity (Table 3.6). The structures for the bioactive compounds are shown in Appendix 1.

Both deletion pools identified wortmannin, methotrexate, clotrimazole, dequalinium dichloride, and dequalinium analog (C-14 linker) as biologically active. The PD-DMA and the DMA has sensitivities that were not significantly different for wortmannin, methotrexate and dequalinium analog (C-14 linker). Dequalinium dichloride however, was more potent in the DMA than the PD-DMA. It is possible that dequalinium dichloride enters the yeast cell via a plasma membrane transporter/s rather than through passive diffusion. Several compounds identified by Lanthaler et al. to require plasma membrane transporters for cell entry (77). Pdr1p and Pdr3p may play a role in upregulation of such a transporter(s), as for example is the upregulation of hexose transporters (31). Inactivation of hexose transporters, Htx9p and Htx11p leads to multidrug resistance phenotype (34).

The PDR substrates identified exclusively by the PDR-deficient strains are $(\mathrm{R}, \mathrm{R})$-cisdiethyl tetrahydro-2,8-chrysenediol, (S)-(+)-camptothecin, (Z)-gugglesterone, 2-(alphanaphthoyl) ethyltrimethyl ammonium iodide, 3,4-dichloroisocoumarin, 4chloromercuribenzoic acid, ammonium pyrrolidinedithiocarbamate, bay 11-7085, calcimycin, cantharidic acid, cantharidin, diphenyleneiodonium chloride, idarubicin, ketoconazole, meclofenamic acid sodium, N-p-tosyl-L-phenylalanine chloromethyl ketone, ruthenium red, tyrphostin A9, tyrphostin AG 879 and Z-L-phe chloromethyl ketone. Known antifungals ketoconazole and clotrimazole were both identified as biologically active in the PD-DMA and only clotrimazole and not ketoconazole demonstrated bioactivity in the DMA. Furthermore, both ketoconazole and clotrimazole 
were completely inhibitory in the PD-DMA. This shows that PDR-deficient strains are better at identifying bioactives. 


\begin{tabular}{|c|c|c|c|}
\hline & Compound & $\begin{array}{l}\Delta \mathrm{xxx} \text { deletion } \\
\text { mutants }\end{array}$ & $\begin{array}{l}\text { HS-deletion } \\
\text { mutants }\end{array}$ \\
\hline 1 & $\begin{array}{l}\text { (R,R)-cis-Diethyl tetrahydro-2,8- } \\
\text { chrysenediol }\end{array}$ & $\mathrm{N} / \mathrm{I}$ & 1 \\
\hline 2 & (S)-(+)-Camptothecin & $\mathrm{N} / \mathrm{I}$ & 4 \\
\hline 3 & (Z)-Gugglesterone & $\mathrm{N} / \mathrm{I}$ & 3 \\
\hline 4 & $\begin{array}{l}\text { 2-(alpha-Naphthoyl)ethyltrimethyl } \\
\text { ammonium iodide }\end{array}$ & $\mathrm{N} / \mathrm{I}$ & 3 \\
\hline 5 & 3,4-Dichloroisocoumarin & $N / I$ & 3 \\
\hline 6 & 4-Chloromercuribenzoic acid & $\mathrm{N} / \mathrm{I}$ & 4 \\
\hline 7 & Ammonium pyrrolidinedithiocarbamate & $\mathrm{N} / \mathrm{I}$ & 3 \\
\hline 8 & Bay $11-7085$ & $\mathrm{~N} / \mathrm{I}$ & 3 \\
\hline 9 & Calcimycin & $\mathrm{N} / \mathrm{I}$ & 1 \\
\hline 10 & Cantharidic Acid & $\mathrm{N} / \mathrm{I}$ & 3 \\
\hline 11 & Cantharidin & $\mathrm{N} / \mathrm{I}$ & 3 \\
\hline 12 & Clotrimazole & 3 & 1 \\
\hline 13 & Dequalinium analog (C-14 linker) & 1 & 1 \\
\hline 14 & Dequalinium dichloride & 2 & 3 \\
\hline 15 & Diphenyleneiodonium chloride & $\mathrm{N} / \mathrm{I}$ & 4 \\
\hline 16 & Idarubicin & $\mathrm{N} / \mathrm{I}$ & 4 \\
\hline 17 & Ketoconazole & $\mathrm{N} / \mathrm{I}$ & 1 \\
\hline 18 & Meclofenamic acid sodium & $\mathrm{N} / \mathrm{I}$ & 4 \\
\hline 19 & Methotrexate & 3 & 3 \\
\hline 20 & $\begin{array}{l}\text { N-p-Tosyl-L-phenylalanine chloromethyl } \\
\text { ketone }\end{array}$ & $N / I$ & 2 \\
\hline 21 & Ruthenium red & $\mathrm{N} / \mathrm{I}$ & 4 \\
\hline 22 & Tyrphostin A9 & $\mathrm{N} / \mathrm{I}$ & 3 \\
\hline 23 & Tyrphostin AG 879 & $\mathrm{~N} / \mathrm{I}$ & 3 \\
\hline 24 & Wortmannin from Penicillium funiculosum & 1 & 1 \\
\hline 25 & Z-L-Phe chloromethyl ketone & $N / \mathrm{I}$ & 2 \\
\hline
\end{tabular}


Table 3.6 - PDR-deficient mutants identifies more bioactives from the LOPAC library. The LOPAC library comprising 1280 pharmacologically active compounds was screened in a growth inhibitory assay against pools of PD-DMA $(\Delta p d r 1 \Delta p d r 3 \Delta x x x)$ and the DMA $(\Delta x x x)$. Compounds that inhibited growth by more than $10 \%$ in three independent screens were identified as biologically active. Bioactivity was ranked from 1-4: $1=0-25 \%$ residual growth, $2=25-50 \%$ residual growth, $3=50-75 \%$ residual growth, $4=75-90 \%$ residual growth and $\mathrm{N} / \mathrm{I}$ denotes residual growth greater than 90\%. The PD-DMA identified 25 bioactive compounds and DMA in the wild-type background identified five bioactive compounds that were also identified by the PD-DMA. The five compounds identified by the DMA were more potent in the PD-DMA with the exception of dequalinium dichloride.

\subsubsection{Conformation of bioactives of LOPAC screen.}

The pool of PD-DMA ( $\Delta p d r 1 \Delta p d r 3 \Delta x x x)$ was used in a dose response assay against two of the bioactive compounds identified from the LOPAC library, camptothecin and ketoconazole. Growth was determined by measuring OD and was compared against growth of the DMA $(\Delta x x x)$ (Fig. 3.13). Both deletion pools were sensitive to camptothecin, but differed in their sensitivities (Fig. 3.13a). At the concentrations of camptothecin screened in the dose response assay, complete inhibition was not observed for either the PD-DMA or the DMA. The PD-DMA however, was significantly more sensitive to camptothecin $\left(\mathrm{IC}_{50} \sim 9 \mu \mathrm{M}\right)$ than the DMA $\left(\mathrm{IC}_{50} \sim 31.62\right.$ $\mu \mathrm{M})$. The difference in sensitivity between the two deletion pools was approximately 3 fold. This is consistent with the findings from the LOPAC screen where $20 \%$ inhibition was observed for the PD-DMA and no growth inhibition for the DMA at 10 $\mu \mathrm{M}$ camptothecin used in the assay. 
Similarly, both deletion pools demonstrated sensitivity to ketoconazole (Fig. 3.13b). The PD-DMA is significantly more sensitive to ketoconazole $\left(\mathrm{IC}_{50} \sim 0.80 \mu \mathrm{M}\right)$ than DMA ( $\left.\mathrm{IC}_{50} \sim 20.54 \mu \mathrm{M}\right)$ corresponding to a 25 fold difference in sensitivity. This is also consistent with the findings for the LOPAC screen where nearly complete growth inhibition was observed for the PD-DMA and no growth inhibition for the DMA at 10 $\mu \mathrm{M}$ ketoconazole used in the assay.
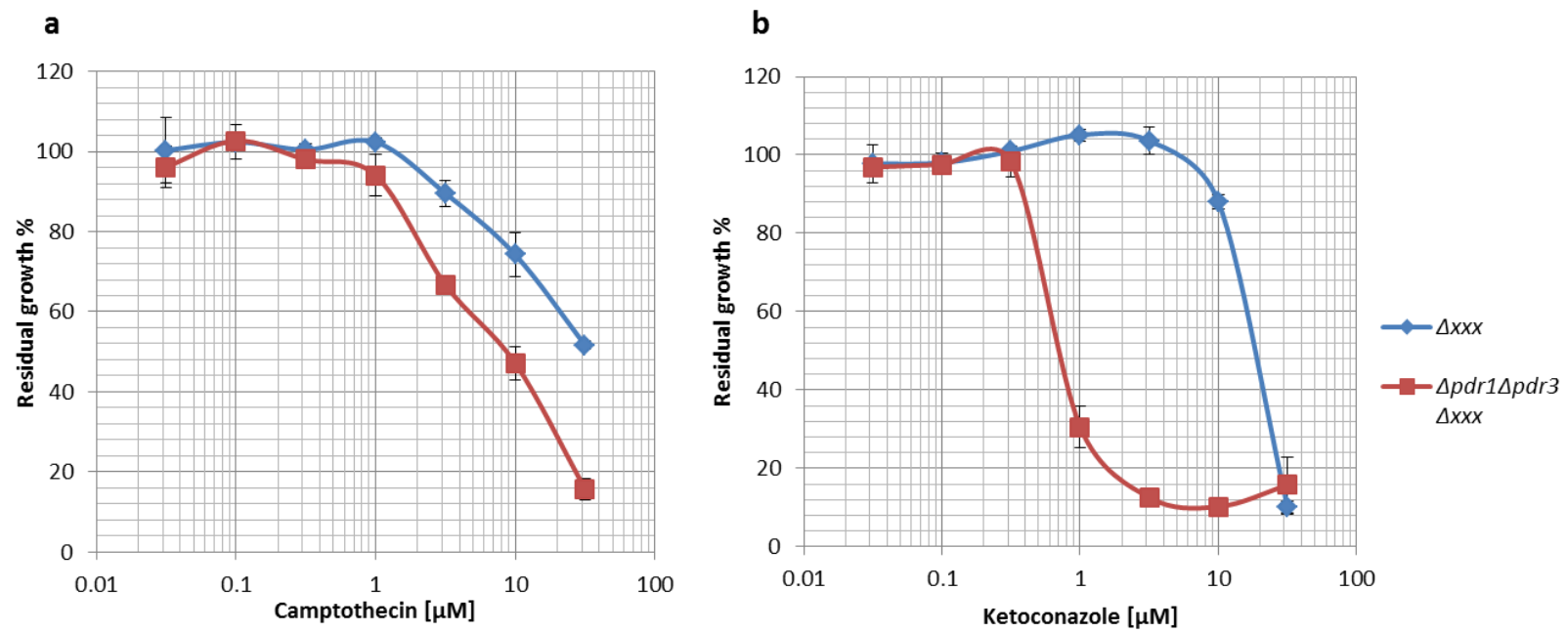

Figure 3.13 - Conformation of bioactives of LOPAC screen. A dose response assay was performed on pools of PD-DMA ( $\Delta p d r 1 \Delta p d r 3 \Delta x x x)$ and DMA $(\Delta x x x)$, against biologically active compounds identified from the LOPAC screen: camptothecin and ketoconazole. (a) The PDDMA was more sensitive to camptothecin than the DMA in the wild-type background. (b) The PD-DMA was more sensitive to ketoconazole than the DMA. 


\section{$\underline{\text { Discussion }}$}

3.9 The $\Delta p d r l \Delta p d r 3$ construct and functional conformation.

In the above chapter the strain $\Delta p d r l \Delta p d r 3$ was constructed by PCR mediated disruption of PDR1 and PDR3 loci with the selectable makers natR and URA3 (Fig. 3.1 \& 3.2). The $p d r 1 \Delta::$ natR $p d r 3 \Delta:: U R A 3$ gene deletions were validated by PCR amplification of the correct size PCR products for both the 5' and 3' flanking regions of the $\operatorname{pdr} 1 \Delta:: n a t R$ and $p d r 3 \Delta:: U R A 3$ constructs (Fig. 3.3).

The PDR-deficient strain, $\Delta p d r l \Delta p d r 3$, was functionally validated by its sensitivity to compounds with well characterised targets in yeast; cycloheximide and rapamycin in a dose response assay (Fig 3.4). Cycloheximide is an inhibitor of eukaryotic translation and it serves as a substrate for the ABC drug efflux transporter, Pdr5p (47, 76). Pdr5p, along with other $\mathrm{ABC}$ drug efflux transporters, form a PDR network which are under the control of two master regulators, Pdr1p and Pdr3p (31). The loss of Pdr1p and Pdr3p therefore, would lead to a decrease in expression of the cycloheximide efflux transporter Pdr5p.

The wild-type yeast strain showed the most resistant to cycloheximide ( $\mathrm{IC}_{50} 122.33$ nM) due to normal Pdr5p mediated drug efflux conferred by functional Pdr1p and Pdr3p transcription factors. The deletion of $\Delta p d r l$ conferred increased sensitivity to cycloheximide $\left(\mathrm{IC}_{50}\right.$ of $\left.84.43 \mathrm{nM}\right)$. This is consistent with a decrease in expression of PDR5 due to loss of one of its major transcriptional regulators (36). Loss of both Pdr1p and $\operatorname{Pdr} 3 p$ transcriptional regulators in the $\Delta p d r 1 \Delta p d r 3$ strain further sensitised it to 
cycloheximide $\left(\mathrm{IC}_{50} \sim 53.90 \mathrm{nM}\right)$ which is consistent with even a further reduction in PDR5 expression (36).

In contrast, rapamycin, a cell cycle regulation inhibitor (78) is not a substrate for the PDR drug efflux transporters and its bioactivity is unaffected by deletions of the PDR1 and PDR3 loci (Fig 1.4b). The similar sensitivity of the three yeast strains to rapamycin confirms that the altered sensitivity observed for cycloheximide among mutant strains were due to the loss of either PDRl or both PDR1 and PDR3 genes. This functional assay confirms that the PDR-deficient strain, $\Delta p d r 1 \Delta p d r 3$, is carrying null mutations in PDR1 and PDR3 loci.

Pdr1p and Pdr3p respond to different environmental cues. Pdr1p is the major transcriptional up-regulator of PDR5 and is required for environmentally induced expression of the PDR network genes, whereas Pdr3p is highly induced in response to mitochondrial defects (37). Using Northern blot analysis, Wolfger et al. demonstrated minor reductions in PDR5 mRNA levels in response to loss of either PDR1 or PDR3, while the loss of both PDRI and PDR3 lead to a significant reduction in PDR5 expression (36). Similar results were observed by Katzmann et al. using PDR5 betagalactosidase reporter assay where loss of either PDRI or PDR3 had minor effects on Pdr5p expression while loss of both $P D R 1$ and $P D R 3$ lead to a major reduction in Pdr5p (79). The PDR5-lacZ expression in the $\Delta p d r 1 \Delta p d r 3$ double mutant was reduced to $2 \%$ of what is observed in wild-type (79). 
These findings can explain the observations above where yeast with wild-type Pdr1p and Pdr3p transcription factors demonstrated greater resistance to Pdr5p substrate cycloheximide. The transcriptional upregulation of ABC drug efflux transporter Pdr5p by Pdr1p and Pdr3p permits greater resistance cycloheximide. The loss of Pdr1p leads to decrease in resistance to cycloheximide through decrease in the expression of the cycloheximide efflux transporter Pdr5p, whereas loss of both Pdr1p and Pdr3p lead to major reduction in of cycloheximide efflux transporter Pdr5p leading to further reduction in cycloheximide resistance.

\subsection{Loss of $P D R 1$ and $P D R 3$ does not activate oxidative stress response}

The loss of Pdr1p and Pdr3p transcription factors does not completely abrogate Pdr5p expression (79). Pdr5p is also under the control of another transcription factor, the oxidative stress response regulator, Yap1p (34). Yap1p is a key regulator of multi-drug resistance as over expression of Yap1 confers resistance several xenobiotics which involve PDR and Major facilitator superfamily (MFS) transporters (80). Therefore cross talk between Yap1p and master regulator of the PDR network, Pdr1p and Pdr3p has been suggested. Yap1p mediates transcriptional control over the several PDR and MFS transporters in response to oxidative stress, namely, the major broad spectrum drug efflux transporter, Pdr5p, and its functional homolog SNQ2, and MFS protein FLR1 which are also targets of Pdr1p and Pdr3p $(34,40)$.

To determine if the oxidative stress response regulator Yap1p is activated in the presence of xenobiotics or to compensates for loss of Pdr1p and Pdr3p for 
transcriptional regulation of PDR and MFS genes, the wild-type strain, YAP1-GFP, and the PDR-deficient strain, $\Delta p d r 1 \Delta p d r 3$ YAP1-GFP was treated with the Pdr5p substrate latrunculin-A, rapamycin which is not a PDR substrate and the known ROS generating compounds menadione and hydrogen peroxide (Fig. 3.9). Cycloheximide was not employed in this assay as it inhibits protein synthesis (76).

Normal oxidative stress response leading to immediate nuclear localisation of cytoplasmic Yap1p was observed for both wild-type strain, YAP1-GFP, and the PDRdeficient strain, $\triangle p d r 1 \triangle p d r 3 Y A P 1-G F P$, following treatment with either menadione or hydrogen peroxide. This suggested that the activation of Yap1p has no requirement of Pdr1p and Pdr3p transcription factors.

In the absence of stress induced by xenobiotics and ROS generating compounds, Yap1p was cytoplasmic in both the wild-type strain and the PDR-deficient strain. This suggests that induction of Yap1p is not required in the absence of xenobiotics or oxidative stress. The absence of Yap1p nuclear localisation in the PDR-deficient strain suggest, Yap1p does not compensate for the loss of Pdr1p and Pdr3p transcription factors in the absence of xenobiotics.

Nuclear localisation of Yap1p was not observed for the wild-type or the PDR-deficient strain following treatment with rapamycin which is not a PDR substrate. However, the treatment with the Pdr5p substrate, latrunculin-A was expected induced Yap1p in the wild-type strain since PDR5 is widely assumed to be positively controlled Yap1p (30, 
34). Therefore, greater induction of Yaplp was expected for the PDR-deficient strain since loss of Pdr1p and Pdr3p leads to a major reduction in Pdr5p (79). However, no Yap1p induction was observed with latrunculin-A in the presence of Pdr1p and Pdr3p transcription factors or in their absence. The findings from the Yap1p reporter suggest that Yap1p does not play a role in PDR5 mediated drug efflux. Furthermore, the oxidative stress response was not induced in the presence of PDR5 substrates even under PDR-deficient conditions where expression level of PDR5 is significantly reduced. Therefore, the activation of oxidative stress response regulator Yap1p is independent of PDR mediated drug resistance.

The overlap in substrate specificity across the drug efflux transporters adds an additional level of complexity to PDR phenotype. The PDR5, SNQ2 and YOR1 overlap in their specificity for substrates as shown by Rogers et al. in a study screening 349 bioactive compounds against gene deletions mutants of $\Delta p d r 5, \Delta s n q 2, \Delta y o r 1$ and $\Delta p d r 1 \Delta p d r 3$ (47). Flr1p is one of 20 MFS proteins with overlapping function with PDR transporters. It mediates efflux of the Pdr5p substrates cycloheximide and fluconazole (81). In the absence of the high affinity transporters, the expression of low affinity transporters are highly induced thereby conferring drug resistance (37). The inactivation of any of the major PDR transporters SNQ2, YOR1 or PDR5 leads to induction of remaining paralogs even in the absence of xenobiotics (37). The deletions of YORI or SNQ2 confers greater drug resistance to Pdr5p substrate cycloheximide than its isogenic wild-type strain (37). The deletion of either $S N Q 2$ or PDR5 confers greater drug resistance to Yor1p substrate oligomycin than wild-type and the loss of both confers greater resistance to oligomycin than the single deletions alone (37). 
In the event that Pdr5p expression is reduced or lost, it is apparent that low affinity transporters are capable of reducing the stress induced by Pdr5p substrates to some extent (37). The additional increased sensitivity observed for cycloheximide in response to loss of both $\Delta p d r 1$ and $\Delta p d r 3$ maybe additional to the down regulation of Pdr5p alone. The loss both $\Delta p d r 1$ and $\Delta p d r 3$ may down regulate the expression of other Pdr1p and Pdr3p inducible low affinity cycloheximide efflux transporters such as FLRI (41). These observations taken together suggest that the loss of both Pdr1p and Pdr3p leads to a down regulation of Pdr5p drug efflux transporter as well as other low affinity cycloheximide transporters.

\subsection{Loss of PDR1 and PDR3 does not induce or impair UPRE response.}

Pdr1p and Pdr3p transcription factors have shown to bind to genomic regulatory elements and modulate the expression of a large array of genes including genes involved plasma membrane composition namely, ABC transporters, MFS and other permeases, genes involved in lipid metabolism, and cell wall synthesis. It is possible these all play a role in reducing the intracellular accumulation of xenobiotics (40).

Genes active in the secretory pathway are required for correct localisation of plasma membrane protein, consequently, deletions mutants of secretory pathway proteins cause sensitisation to multiple compounds (82). EMC1 and EMC4 are two of six members of the ER membrane complex (EMC) which are required for normal protein folding and loss EMC1-6 genes leads to incomplete blockage of proteins secretion at the ER and 
induced UPR $(70,83)$. Transcriptional upregulation EMC1 and EMC4 by Pdr1p and Pdr3p under induced conditions suggest an active role of Pdr1p and Pdr3p in protein folding and secretion $(84,85)$. This led to the suggestion that the loss of Pdr1p and Pdr3p may lead to an induction of UPR to deal with toxic misfolded proteins. However, in the current work normal UPR induction was observed with both wild-type strain and PDR-deficient strain with the UPR inducing compounds DTT and tunicamycin (Fig. 3.8). The UPRE expression levels increased in dose dependent manner with both of DTT and tunicamycin and no significant difference was seen between the wild-type strain and PDR-deficient strain. Additional induction of UPRE was not observed for the PDR-deficient strain relative to wild-type. Under untreated conditions, wild-type strain and the PDR-deficient strain had similar UPRE expression levels which suggest that loss of Pdr1p and Pdr3p does not induce UPR. This shows that the down regulation of EMC1 and EMC4 by loss of Pdr1p and Pdr3p transcription factors was not enough to induce the UPR. Thus, one or more of the other transcriptional regulators of EMC1 (Phd1p and Yap5p) and EMC4 (Gcn4p, Ino4, Msn2p and Tos8) (86) may influence their expression more significantly.

\subsection{SGA analysis}

The query strain $p d r 1 \Delta:: n a t R$ pdr3 $\Delta:: U R A 3$ was robotically mass mated against the DMA which comprises the non-essential single deletion mutants of yeast, and put through a series of SGA selection steps (Chapter 2 Materials and Methods) to generate $\sim 4700$ triple mutants in the background of null mutations in PDRI and PDR3 loci. The colony sizes were assessed for phenotypic enhancements using Colony HT and SESA 
(Chapter 2 Materials and Methods) and 43 phenotypic enhancements were identified for $\Delta p d r 1 / \Delta p d r 3$ double mutant.

26 genes were members of the linkage group of the query genes $\Delta p d r 1$ and $\Delta p d r 3$ and thus were not genuine PEs. The linkage group arises when genes are in close proximity on the same chromosome such that their assortment is not independent of each other and they are inherited together (linkage disequilibrium). As the distance between the two selection markers decreases, the recombination frequency between the markers also decreases. This leads to reduced number of triple mutant meiotic progeny for deletion mutants of contiguous genes located on either side of the query gene that show reduced growth. Nine genes were in linkage disequilibrium with $\Delta p d r 3$ and 17 were in linkage disequilibrium with $\Delta p d r l$. Linkage disequilibrium surrounding $\Delta p d r 1$ is larger due to its closer proximity to the centromere, thus inhibiting the recombination frequency further.

The four genes URA1, URA2, URA4 and URA5 required for pyrimidine biosynthesis were identified as phenotypic enhancements in the SGA since CaURA3 was used as a selection marker for $\Delta p d r 3$ deletion. In the absence of uracil, the genes $U R A 1, U R A 2$, URA3, URA4 and URA5 are required to convert L-glutamine (generated through alanine, aspartate and glutamate metabolism) and carbamoyl phosphate (generated through arginine and proline metabolism) to uradine monophosphate (UMP). URA6 converts UMP to uradine diphosphate (UDP) which is subsequently converted to uradine triphosphate (UTP) by YNK1 which is incorporated into RNA. The CaURA3 gene complements the $\Delta u r a 3$ deletion carried by the parental strains but requires $U R A 1$, 
$U R A 2, U R A 4$ and $U R A 5$ genes in the uracil biosynthesis pathway to function as a uracil prototroph and to permit selection on media lacking uracil (87). In the presence of uracil, FUR1 converts uracil to UMP which is subsequently converted to UDP and to UTP and is incorporated into RNA. The $\Delta f u r l$ mutant is therefore, synthetic lethal with $\Delta u r a 3$ and was employed as a synthetic lethal control for random spore analysis.

The genes STE20, FARl and MFAl were also identified as phenotypic enhancements in two independent SGAs. STE20 is an essential component of the yeast pheromone response and required for pheromone induced cell cycle arrest at G1 and pheromone induced expression of mating specific genes (71). Loss of STE2O from haploid cell results in unresponsiveness to pheromone, therefore, inability to form mating specific morphological changes such as shmoo development. The loss of STE20 from haploid yeast cells result in inability to mate and therefore, was identified as a false phenotypic enhancement in SGA methodology (71).

The gene FAR1 is responsible for cell cycle arrest at G1 via inhibition of the cyclin dependent kinase (CDK) complex in response to alpha factor and also is required for pheromone induced polarised growth toward the mating partner which is independent of cell cycle arrest. Both of which are required for efficient mating. Loss of FARl from haploid yeast results in inability to locate the mating partner thus, leading to reduced mating efficiency (72). 
MFAl is another gene required for normal mating efficiency. MFAl and its functionally redundant homolog MFA2 encodes for mating factor-a which is required for conjugation of MATa and MAT $\alpha$ cells (73). The reduced levels of mating factor-a in the absence of MFAl are adequate for cell cycle arrest at G1, shmoo formation and paring of mating cells but not sufficient to induce cell fusion in mating MATa cells. (88). $\Delta m f a l$ was identified as synthetic lethal in SGA with wild type MATa by Tong et al. but not MFA2 but results here agree with the requirement of MFA1 for normal mating (11). It may be concluded therefore that STE20, FARI and MFA1 are required for efficient mating between MATa and MAT $\alpha$ cells. Deletion mutants lacking these genes could therefore give rise to the appearance of PEs in SGA due their inability to generate sufficient number of diploids for sporulation and the PE are independent of the $\Delta p d r 1 \Delta p d r 3$ deletions.

\section{$\underline{3.13 \text { Random spore analysis }}$}

The nine remaining putative phenotypic enhancements $(\Delta \operatorname{mir} 1, \Delta m r m 2, \Delta \operatorname{rad} 14, \Delta s o r 9$, $\Delta y l r 346 c, \Delta y i l 012 w, \Delta y p r 126 c, \Delta r p l 9 a$ and $\Delta z r c 1)$ and the mating deficient mutants ( $\Delta m f a l, \Delta$ farland $\Delta$ ste 20 ) were independently mated against the PDR-deficient strain, $\Delta p d r 1 \Delta p d r 3$ to validate phenotypic enhancements. The resulting heterozygous diploids with were sporulated and the meiotic progeny were assessed for phenotypic enhancement using random spore analysis.

The three previously characterised mating deficient mutants $(\Delta m f a l, \Delta$ farland $\Delta$ ste 20$)$ in addition to $\Delta r p l 9 a, \Delta y i l 012 w$ and $y p r 126 c$ displayed reduced mating efficiency with 
the PDR-deficient strain. For the explained above $\Delta m f a l, \Delta$ farland $\Delta$ ste20 mutants need no further discussion. Following sporulation, six out of the nine remaining deletion strains displayed reduced sporulation efficiencies with PDR-deficient strain including the three mating defective genes ( $\Delta r p l 9 a, \Delta y i l 012 w$ and $y p r 126 c)$ and three additional strains $(\Delta m r m 2, \Delta \operatorname{rad} 14, \Delta \operatorname{sor} 9)$.

The six deletion mutants ( $\Delta m r m 2, \Delta$ rad14, $\Delta$ sor9, $\Delta y p r 126 c, \Delta y i l 012 w$ and $\Delta r p l 9 a)$ displaying reduced mating/ sporulation efficiency with the PDR-deficient strain were mated against $\Delta u r a 3$ control strain and sporulated to identify whether $\Delta p d r 1 \Delta p d r 3$ contributed to the poor mating efficiency or poor sporulation trait. As with the PDRdeficient strain, the three deletion mutants $(\Delta m r m 2, \Delta \operatorname{rad} 14, \Delta$ sor9) were mating deficient with $\Delta u r a 3$ and all six sporulation defective deletion strains ( $\Delta m r m 2, \Delta r a d 14$, $\Delta$ sor9, $\Delta y p r 126 c, \Delta f a r l, \Delta y i l 012 w$ and $\Delta r p l 9 a)$ displayed reduced sporulation efficiency with $\Delta u r a 3$ control cross. The results were identical to mating and sporulation efficiencies observed with PDR-deficient strain. This suggests that the reduced mating efficiency was not contributed by the $\Delta p d r 1 \Delta p d r 3$ double mutations and was falsely identified due to reduced number of diploids and spores generated during the SGA.

The three remaining deletion strains, $\Delta$ mirl, $\Delta$ rad14 and $\Delta y l r 346 c$ had normal sporulation efficiencies with the PDR-deficient strain. The deletion mutants, $\Delta m r m 2$ and $\Delta \operatorname{rad} 14$ although deficient in mating, sporulated efficiently with both $\Delta p d r 1 \Delta p d r 3$ double mutant and the $\Delta u r a 3$ deletion mutant. The deletion mutant $\Delta y \operatorname{lr} 346 c$ had normal mating and sporulation efficiencies with PDR-deficient strain. The currently 
uncharacterised protein encoded by $Y L R 346 c$ was of interest as it contains a PDR responsive element within its promoter and has shown to be highly induced by hyperactive and constitutively expressed variants of both Pdr $1 p$ and Pdr3p transcription factors $(40,84,89,90)$. Interestingly, $\Delta y \operatorname{lr} 346 c$ did not show PE with $\Delta p d r 1 \Delta p d r 3$ double mutant. This suggests that either YLR346C is not necessary under PDR-deficient conditions or another transcription factor plays a major role in transcriptional regulation of YLR346C.

The gene expression levels of $M I R I$ and $R A D 14$ were not altered by constitutively activated alleles of Pdr1p and Pdr3p (40, 84, 89, 90). MIR1 encodes a mitochondrial phosphate carrier within the inner membrane which catalyses the proton co-transport of inorganic phosphate from the inner membrane to the mitochondrial matrix and is functionally redundant with PIC2 (91). RAD14 encodes for subunit of nucleotide excision repair factor NEF1 which incises the 5' end of the damaged DNA during nucleotide excision repair (92). Neither MIR1 nor RADI4 demonstrated phenotypic enhancement with $\Delta p d r l \Delta p d r 3$ double mutant when individually assayed.

Interestingly, the YPR126C coding region overlaps $Y L H 47 / Y P R 125 \mathrm{~W}$ which encodes a mitochondrial membrane protein that interacts with mitochondrial ribosomes (discussed in SGD). Similarly, the coding region of YILO12W is adjacent to PDR11/YILO13C, a target of Pdr1p which mediates sterol uptake when sterol biosynthesis is impaired (93). However, neither $\Delta y l h 47$ nor $\Delta p d r 11$ demonstrated a PE with $\Delta p d r 1 \Delta p d r 3$ double mutant in the SGA. This suggests that $\Delta y \operatorname{pr} 126 c$ and $\Delta y i l 012 w$ may have fallen out due to pinning errors during the SGA procedure. 
From the nine putative PE identified with $\Delta p d r 1 \Delta p d r 3$ double mutant in the SGA analysis, none were confirmed as true PE by random spore analysis. This was not expected since, on average 34 phenotypic enhancements are observed with pairwise deletions when using a single query gene (94). This number should be significantly more when a double disruptant mutant is used as query strains to generate triple mutants. This suggests the Pdr1p and Pdr3p transcription factors are not necessary for viability in the conditions used in the random spore analysis. More importantly, Only 73 deletion strains were lost in the construction of the PD-DMA as results of mating and sporulation deficiencies, pinning errors and extreme linkage disequilibrium. Therefore, the PD-DMA gives near comprehensive coverage of the non-essential haploid DMA. The PD-DMA can therefore be used in the place of the wild-type background DMA for chemical-genetic profiling assays and also in SGA analysis as the MATa DMA to introduce query mutations.

\subsection{Deletion mutants sensitive to rapamycin}

The PD-DMA comprising the non-essential mutants were pinned on to SC agar plates (solid phase growth) containing $5 \mathrm{nM}$ rapamycin $\left(\sim \mathrm{IC}_{30}\right)$. Three independent solid phase chemical genetic profiling experiments identified 18 phenotypic enhancements.

Rapamycin is a lipophilic macrolide which binds and inhibits TORC1, a Ser/Thr protein kinase localised predominantly in the vacuolar membrane where it responds to the quality and the abundance of the nitrogen and carbon sources within the vacuole (95). 
TORC1 positively regulates anabolic processes of protein synthesis and translation initiation and negatively regulates catabolic processes, that promote nitrogen assimilation under nutrient starvation conditions such as the nitrogen discrimination pathway, amino acid biosynthesis, general amino acid permease, retrograde transport and macroautophagy (95-99).

TORC1 inhibition by rapamycin mimics nutrient starvation and promotes downregulation of anabolic processes while promoting catabolic pathways to assimilate alternative sources of nitrogen which consequently, leads to a total reduction in energy consumption leading to cell cycle arrest at G1/S and entry into quiescence $(96,97,100)$. The deletion strains hypersensitive to rapamycin in the DNA barcode microarray were indicative of TORC1 inhibition (Table 3.4 and Fig. 3.11). The biological processes affected by rapamycin treatment include negative regulation of autophagy, negative regulation of cellular catabolic process, endosomal transport and retrograde transport from endosome to Golgi. Several genes encoding for proteins in multi-protein complexes, TORC1 (TOR1), GARP (VPS53 and VPS51) and GSE (GTR1 and GTR2) were also identified.

Previously only gene deletion mutants of TOR1, PHO80, GTR1, GTR2, PEP8 and ECM8 were identified to demonstrate hypersensitivity to rapamycin in high throughput screens and were among the top 105 hypersensitive strains (PEs ranked as strong sensitivity) with rapamycin (82). Interestingly gene deletion mutants of CKA2, EOS1, ERG2, OPI3, PKR1, SSD1, TRR2, VPS27, VPS51, VPS53, YDL121C and YEL057C 
have not been previously been identified as PE with rapamycin in a high throughput screen.

Some of these PE are discussed below. The gene $C K A 2$ encodes for the alpha catalytic subunit of multi protein complex protein kinase CK2, which is required for cell cycle progression (101). It has been shown that, CK2 mutants affect the expression of PHO pathway genes including $\mathrm{PHO80}$, the key cyclin in the phosphate response pathway (a previously identified rapamycin hypersensitive strain) (101). The protein encoded by SSD1 mediates cell integrity and is a target of Tap2p a downstream effector of TORC1 (102). Proteins encoded by ERG2 and $O P I 3$ are involved in lipid metabolism and are likely to encode multi-drug resistance genes, as loss of ergosterol increases the plasma membrane permeability to compounds (29). VPS51 and VPS53 encode for the GARP complex which regulates two distinct pathways of retrograde transport, a catabolic pathway that is induced in response to TORC1 inhibition $(103,104)$. GARP complex mediates the fusion of both early-endosomal and late-endosomal vesicles with the transGolgi network and GARP complex mutants are impaired in protein recycling and sorting, therefore, it may be that the localisation of various membrane proteins effected including TORC1 $(103,105)$.

\section{$\underline{3.15 \text { Deletion mutants hypersensitive to cycloheximide. }}$}

The sensitivity of the gene deletion strains to cycloheximide was assessed in a DNA barcode microarray experiment (Chapter 2 Materials and Methods) utilizing the haploid PDR-deficient mutants. The competitive hybridisation of Cy3 (control) and Cy5 (cycloheximide treated) labelled barcodes to the microarray chip was used to determine the deletion strains hypersensitive to cycloheximide. 
Cycloheximide is a glutarimide originally isolated from Streomyces griseus, and has been used as an inhibitor of eukaryotic protein synthesis for decades, but it precise mechanism of action however, remained unknown until recently (76). Cycloheximide binds the deacetylated tRNA within the E-site of the ribosome and skews eEF2 mediated translocation of the deacetylated tRNA from the P-site to E-site leading to a stall in translational elongation (76). As it takes two rounds of translocation before the deacetylated tRNA reaches the E-site, cycloheximide permits two rounds of complete translation before stalling translational elongation and this leading to polysome profiles with multiple ribosomes arrested on the mRNA (76). As protein synthesis is a complex process, genes involved in various biological processes were expected to show PEs in a cycloheximide chemical genetic screen.

The DNA barcode microarray using the haploid PDR-deficient mutants identified 31 phenotypic enhancements with cycloheximide which were indicative of translation inhibition and translational elongation inhibition. In addition, several other biological processes were also affected by cycloheximide treatment including transcription and cytoskeletal organisation. Out of the 31 phenotypic enhancements only 11 (RPL23A, MBF1, LEM3, ERG2, CAP1, JLP2, RKR1, SIW14, SKI3, UBX2 and VMS1) were previously identified in high-throughput screens with cycloheximide (82, 106). However, only two ribosomal associated genes were among the top 46 cycloheximide hypersensitive strains ( $\mathrm{P}$ value <0.001) in homozygous diploid microarray in Hillenmeyer et al. which included RPL23A encoding for the large (60s) subunit of the ribosome and $R P P 2 A$ encoding for the ribosomal stalk (106). No ribosomal or 
ribosomal associated genes were among the 51 PEs identified by solid phase chemical genetic profiling screen in Parsons et al. (2004) using the haploid DMA.

In contrast, the haploid deletion mutants in the PDR-deficient background identified $R P L 23 A$ and several additional large ribosomal proteins including RPL9A, RPL36A, RPL13A, RPLAA and RPL37A and several translation elongation genes EFT2, RPP1B and GCN20. These genes have not been previously identified in chemical genetic screens employing cycloheximide.

The large ribosomal subunit catalyses the translocation of the peptidyl moiety from the P-site tRNA to A-site amino-acyl tRNA (107). Expression of RPL9A and RPL36A have previously been shown to upregulate in response cycloheximide treatment $(108,109)$, therefore, genes encoding for the large ribosomal subunit may play a compensatory role in mediating resistance to sub-lethal concentrations cycloheximide.

The genes EFT2, RPPIB and GCN20 mediate the translational elongation step (62). The gene EFT2 encode for eEF2, a translocase promoting the translocation of tRNA with the peptide chain from the A-site to the P-site as well as translocation of deacetylated tRNA from P-site to the E-site; the elongation step inhibited by cycloheximide binding (110). As EFT1 is functionally redundant with EFT2 for eEF2, the reduced levels of eEF2 in the $\Delta e f t 2$ mutant may lead to more severe defect in protein synthesis under cycloheximide treated conditions. 
The protein encoded by $R P P 1 B$ is one of four acidic proteins which forms the ribosomal stalk (RPP2A was identified by the Hillenmeyer et al. (106)) (111). Although acidic proteins are not necessary for ribosomal function, different ribosomal stalk compositions can promote the binding of different translational elongation factors to differentially regulate the translation of various mRNA transcripts (112). As for example, the ribosomal stalk L12 in bacteria is required for binding elongation factors EF-Tu and EF-G to the bacterial ribosome (113). Similar mechanism may be involved in yeast where different ribosomal stalk mutants may have different sensitivities to cycloheximide due to inability to recruit translation elongation factors such as eEF2.

The gene GCN2O is another translational elongation factor that has not previously been associated with cycloheximide resistance. GCN2O interacts with GCN1 to form a translational elongation factor 3 (eEF3) like protein (114). GCN20 shows sequence homology to the $\mathrm{ABC}$ domain of eEF3 and GCN1 shows homology to the region Nterminal of the $\mathrm{ABC}$ domain of eEF3 (114). eEF3 is thought to facilitate the release of tRNA from the E-site of the ribosome after each round of elongation (114). The loss of GCN2O under cycloheximide treated conditions may further impair the release of the deacetylated tRNA bound by cycloheximide with in the E-site of the ribosome. Therefore, Gcn20p may dislodge cycloheximide from the E-site along with the deacetylated tRNA relieving the block in translational elongation to some extent.

At high concentrations cycloheximide also inhibits transcription (76), namely the RNA polymerase II subunit, MBF1 (also identified in the Parsons et al.(82)) and transcriptional co-activator $\mathrm{SOH} 1$. The cytoskeletal organisation also play a role in 
translation as several translation initiation and elongation proteins interact with actin microfilaments (115). The cytoskeletal organisation genes, CAPI and SIW14 were among genes previously identified with cycloheximide chemical screens (106).

Hillenmeyer et al. observed that several deletion mutants particularly, lipid metabolism and vesicle transport mutants to demonstrate hypersensitivity to more than $20 \%$ of the compounds screened (116). These were categorised as multi-drug resistant (MDR) genes in the network diagram with cycloheximide (Fig. 3.2). However, some of these MDR genes may play a specific role in a given compounds mode of action rather than general 'frequent fliers' therefore were not excluded from the results from this study.

\subsection{PDR-deficient mutants identifies more biological active compounds}

The deletion mutants in the PDR-deficient background $(\Delta p d r 1 \Delta p d r 3 \Delta x x x)$ and the deletion mutants in the wild-type background $(\Delta x x x)$ were screened in parallel as a part of pool against the LOPAC library comprising 1280 pharmacologically active compounds in a growth inhibitory assay. The screening of the LOPAC library showed that PDR-deficient strains are more sensitive at identifying biologically active compounds. The PDR-deficient strains identified five times as many compounds as the yeast strains in the wild-type background (Table 3.6).

The $\Delta p d r 1 \Delta p d r 3 \Delta x x x$ mutants were expected to identify more bioactives than the $\Delta x x x$ in wild-type background as the loss of Pdr1p and Pdr3p transcription factors leads to a downregulation in the expression of PDR pumps, which consequently lead to a reduction in the ability to efflux PDR substrates. The PDR-deficient strains therefore, 
identify PDR substrates that would normally be targeted for efflux in the wild-type strains with normal PDR. The increased sensitivity of the PDR-deficient strains to PDR substrates was demonstrated above by the increased sensitivity of the Pdr5p substrate to cycloheximide. The loss of Pdr1p and Pdr3p transcription factors does not alter the bioactivity of compounds that are not PDR substrates as seen by the similar sensitivities to rapamycin by the PDR-deficient strain and the wild-type strain.

The 20 PDR substrates identified exclusively by the PDR-deficient strains included camptothecin and ketoconazole. These were employed in a dose response assay using the DMA and the PD-DMA to validate results from the LOPAC screen. In the dose response assay both camptothecin and ketoconazole demonstrated bioavailability in PDR-deficient and wild-type back ground strains but camptothecin was $\sim 3$ fold more potent and ketoconazole was $\sim 25$ fold more potent in the PDR-deficient background. Both ketoconazole and camptothecin have been previously identified as PDR substrates. Snq2p serves as the major drug efflux transporter for camptothecin while both Yor1p and Snq2p are efflux transporters for ketoconazole $(37,117)$. Thus, validating the results for the LOPAC screen. Furthermore, the increased sensitivity to ketoconazole and camptothecin in the $\Delta p d r 1 \Delta p d r 3$ background is consistent with down regulation in the expression of PDR network genes, YOR1 and SNQ2 due to loss of Pdr1p and Pdr3p transcription factors. This illustrates that deletions of PDRI and PDR3 genes sensitises yeast to compounds that are substrates for various efflux transporters not only for Pdr5p efflux transporter. 


\subsection{The utility of the PDR-deficient strains}

In current biochemical assays, the PDR pump mediated drug efflux is overcome by either adding detergents or using ergosterol biosynthesis mutants to permeabilised cell membranes or by adding more compound (50). Using detergents and ergosterol biosynthesis mutants permits the entry of PDR substrates but can have other undesirable effects. Alternatively PDR pump mediated drug efflux can be overcome by using PDRdeficient strains which are missing either one or more of the drug efflux pumps or transcription factors that upregulate the expression of PDR pumps (49). However these have not yet been incorporated into the genetic background of the DMA to sensitise the entire deletion set prior to the study described in this thesis.

The chemical genetic screens in the current DMA (in wild-type background) for PDR substrates requires incubation of yeast cells with high concentrations of PDR substrates to overcome the PDR mediated efflux thereby triggering other 'damage control' (general stress) mechanisms. The PDR-deficient system described in this chapter induces cell inhibition at lower concentrations without flooding the cellular machinery with compounds that are not readily available which is often the case natural products. Thereby, reducing the effective minimum inhibitory concentration (MIC) as well as off target toxicity. The PDR-deficient system widens the search parameters in this assay, thus, increasing the likelihood of finding biologically active compounds in preliminary biochemical screens in yeast. 


\section{PD-DMA Validation Employing Latrunculin-A}

\section{Introduction}

\subsection{Marine natural products as lead compounds for drug discovery}

Recent efforts to discover compounds with novel scaffolds have led to the study of organisms in unexplored ecological niches, such as myoxbacteria in terrestrial environments, which has generated a wealth of unique natural products which display biological activity (6). Currently, 60-75\% of the proven drugs in the areas of cancer and infectious disease are natural products, or are derived from natural product (118). While the terrestrial environments continue to be a rich source for natural product-based drug discovery, marine niches largely remain a relatively untapped source. Since marine natural products make up only a handful of drugs currently available on the market, they may preserved as potential source of novel compounds with novel mechanisms (118).

The marine ecosystem contains enormous biodiversity, as a vast number of soft-bodied non-motile organisms have evolved various chemical means of defence against predators, competitors, and to acquire prey (119). These natural toxins are highly potent, and are produced in high concentrations, in order to overcome rapid dilution upon release into water (119). In addition, marine organisms are likely to serve as a rich source of compounds with characteristics different from those derived from terrestrial organisms, due to the different environments the two originate from (4). Therefore, focusing on marine organisms will improve the odds of finding new chemical scaffolds (4). These new scaffolds, with novel mechanisms of action, could provide a better 
approach to combating cellular resistance developed against existing scaffolds than that of tailoring the existing scaffolds $(4,6)$.

The evolutionary conservation of genes from micro-organisms to higher mammals rationalises the search of novel compounds derived from marine organisms as drug candidates to treat human disease using semi-inhibitory concentrations (118). Even if some small molecules containing novel chemistry with novel targets do not develop into commercial drugs, they could serve as probes to better understand pathways in disease processes and help development of better drugs in the future (4). The compounds containing novel chemistry can also serve as templates for creating further synthetic derivatives with improved properties over the parental compounds and serve as a starting point for combinatorial chemistry $(4,118)$.

\section{$\underline{4.2 \text { Latrunculin-A }}$}<smiles></smiles>

Figure 4.1 - Structure of latrunculin-A 
Latrunculin-A is marine macrolide originally isolated from red sea sponge Negombata magnifica, and has since been isolated from other taxonomically unrelated species (120). In initial screens, latrunculin-A was shown to bind and sequester monomeric globular actin (G-actin) in rabbit skeletal muscle action in a 1:1 molar complex, thereby preventing the assembly of actin monomers into fibrous actin (F-actin) filaments (121). This was also shown to be true with yeast actin, and furthermore, latrunculin-A was shown to disrupt the actin cytoskeleton by direct binding of actin, as opposed to by promoting F-actin disassembly (122).

Actin filaments, microtubules and intermediate filaments together form the cell cytoskeleton which is a highly dynamic structure constantly undergoing polymerisation and depolymerisation processes (123). In budding yeast, microtubules are utilised only for nuclear migration during mitosis, whereas in animals microtubules are also used for organelle transport $(122,124)$. Latrunculin-A, in contrast to other cytoskeletal disrupting agents such as cytochalasin-B, only interferes with actin microfilament assembly, without altering microtubules $(122,125)$. Latrunculin-A treatment leads to near complete loss of actin cables within 2 minutes, and formation of actin patches (indicative of complete actin depolymerisation) within 5 minutes (122). Following removal of the latrunculin-A, normal polarised actin cytoskeleton is restored in $\sim 60$ minutes (122). However, disassembly of actin patches and actin cables observed following latrunculin-A treatment suggests rapid turnover of actin in yeast cells (122).

The actin cytoskeleton in yeast is utilised in numerous processes, including cytokinesis, endocytosis and exocytosis, organelle transport, vesicle transport, establishment of cell 
polarity, and bud formation (122). Nearly all of these processes (with the exception of cytokinesis) are affected by latrunculin-A treatment (122). Several gene deletion mutants of actin-associated proteins have previously been shown to demonstrate hypersensitivity to latrunculin-A, particularly, $\Delta$ cap2, $\Delta$ sac6, $\Delta s e r 2, \Delta$ sla2 and $\Delta t p m 1$ (122). A DNA barcode microarray experiment in Hillenmeyer et al. (2008) (116) using the wild-type background homozygous diploids identified deletion mutants of cytoskeleton associated genes (AIP1, ARP1, BBC1, BNI5, BNR1, CAP1, CLB2, JNM1, MDM20, PEA2, PFD1, RRD1 and SLM1) within the top 120 (P-value <0.001) hypersensitive deletion strains following latrunculin-A treatment.

Whether this is the whole spectrum of genes in cytoskeletal organisation that would be affected by latrunculin-A was therefore a good question to prove the utility of the PDRdeficient deletion mutant set. Any additional phenotypic enhancements identified in the PDR-background DMA would give further insight in to the action of latrunculin-A. 


\section{$\underline{\text { Results }}$}

4.3 PDR-deficient strains are more sensitive to latrunculin-A.

Pools of DMA $(\Delta x x x)$, and PD-DMA $(\Delta p d r 1 \Delta p d r 3 \Delta x x x)$ were used in a dose response assay employing latrunculin-A. The growth of the PD-DMA was determined by measuring the optical density of the culture following latrunculin-A treatment and was compared against the growth of DMA (Fig. 4.2). The DMA was more resistant to latrunculin-A and had an $\mathrm{IC}_{50}$ of $3.24 \mu \mathrm{M}$, whereas the PD-DMA was less resistant to latrunculin-A, with an $\mathrm{IC}_{50}$ of $0.12 \mu \mathrm{M}$. The PD-DMA demonstrated a $\sim 28$ fold increase in sensitivity to latrunculin-A, thus identifying latrunculin-A as a PDR substrate.

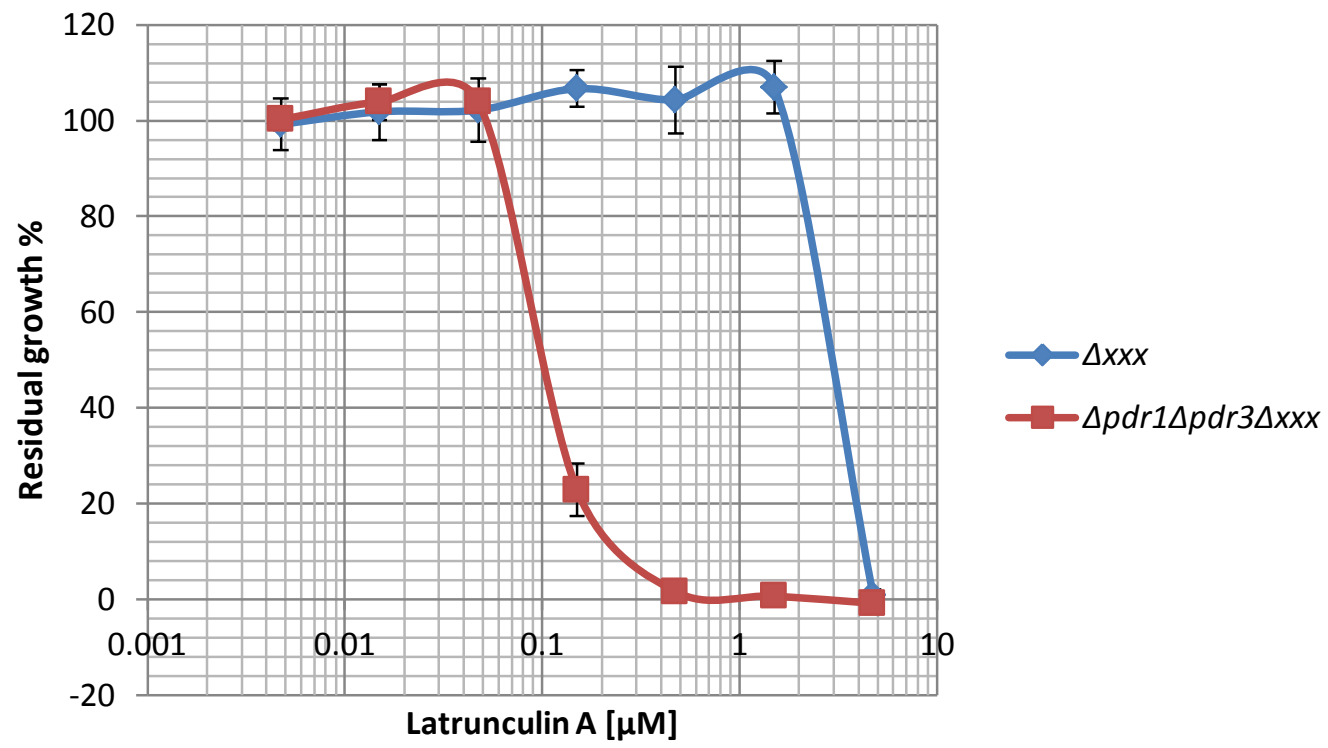

Figure 4.2- PD-DMA are more sensitive to latrunculin-A. In a dose response assay, DMA $(\triangle x x x)$ and PD-DMA ( $\Delta p d r 1 \Delta p d r 3 \Delta x x x)$ pools were assessed in their sensitivity to latrunculin-A. The PD-DMA was the more sensitive to latrunculin-A in comparison to DMA in the wild-type background. 


\subsection{PDR5 is the major efflux transporter for latrunculin-A.}

The single gene deletion strains of the major PDR pumps PDR5, SNQ2 and YOR1 were used in dose response assays employing the PDR substrate latrunculin-A. Growth was determined by measuring culture OD following latrunculin-A treatment and was compared against growth of both a $\Delta$ his 3 control strain and the PDR-deficient strain, $\Delta p d r 1 \Delta p d r 3$ (Fig. 4.3a). The $\Delta p d r 5$ strain was the most sensitive of the PDR pump mutants to latrunculin-A, with an $\mathrm{IC}_{50}$ of $\sim 0.099 \mathrm{nM}$ and its sensitivity was similar to that of the $\Delta p d r 1 \Delta p d r 3$ strain $\left(\mathrm{IC}_{50} 0.089 \mathrm{nM}\right)$ and was $\sim 10.15$ fold more sensitive than that of the $\Delta h i s 3$, wild-type control strain $\left(\mathrm{IC}_{50 \sim} 1.01 \mathrm{nM}\right)$. In contrast, the $\Delta s n q 2$ strain $\left(\mathrm{IC}_{50} \sim 0.99 \mathrm{nM}\right)$ had a sensitivity that was not significantly different from that of the $\Delta$ his3 control strain $\left(\mathrm{IC}_{50} \sim 1.01 \mathrm{nM}\right)$.

Interestingly, the PDR pump mutant $\Delta y o r l$ was resistant to latrunculin-A $\left(\mathrm{IC}_{50} \sim 1.33\right.$ $\mathrm{nM}$ ) in comparison to the $\Delta$ his 3 control (Fig.4.3a -b). This may have resulted from an induction of remaining drug efflux transporters in the absence of YOR1. The similar sensitivities of the $\Delta p d r 5$ and $\Delta p d r 1 \Delta p d r 3$ mutant strains to latrunculin-A identifies Pdr5p as the major drug efflux transporter of latrunculin-A 


\section{$\mathbf{a}$}
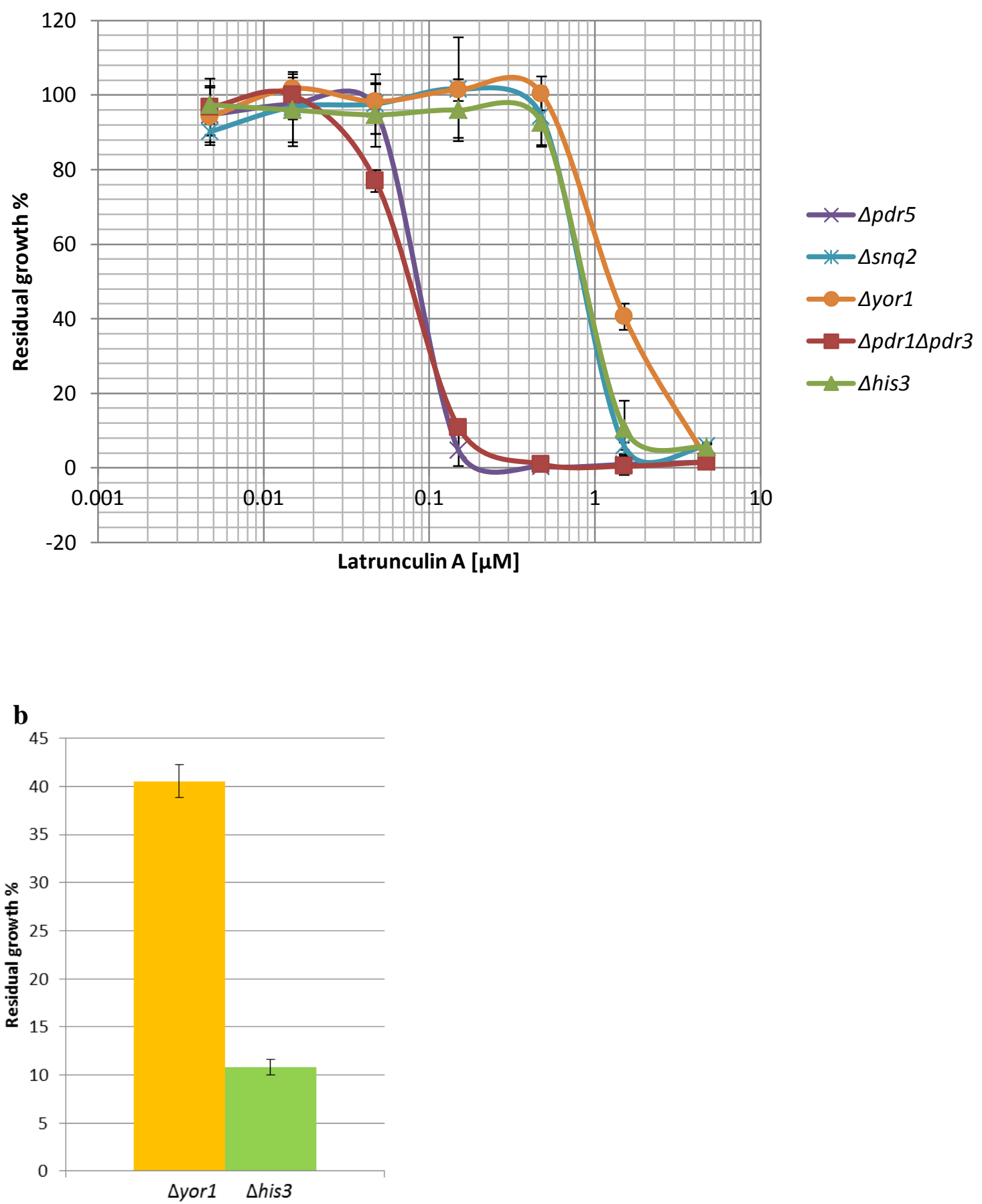

Figure 4.3 - Latrunculin-A is a Pdr5p substrate. (a) Dose response assays were performed on the yeast strains $\Delta p d r 5, \Delta s n q 2, \Delta y o r 1, \Delta h i s 3$, and $\Delta p d r 1 \Delta p d r 3$, against the PDR substrate latrunculin-A. $\Delta p d r 5$ and $\Delta p d r 1 \Delta p d r 3$ strains were the most sensitive to latrunculin-A and had similar sensitivities to each other, followed by $\Delta s n q 2, \Delta h i s 3$ control and $\Delta y o r 1$ strains. (b) 1.5 $\mu \mathrm{M}$ latrunculin-A against PDR pump mutant $\Delta y o r 1$ and $\Delta$ his3 control strain from the above dose response assay as to illustrate the increased resistance of $\Delta y o r 1$ to latrunculin-A relative to $\Delta$ his3 control. 


\subsection{Deletion mutants hypersensitive to latrunculin-A}

The sensitivities of haploid PD-DMA $(\Delta p d r 1 \Delta p d r 3 \Delta x x x)$ to latrunculin-A were assessed as a pool by DNA barcode microarrays (Chapter 2 Materials and Methods) that measures relative fitness of strains in the pool following compound treatment. The competitive hybridisation ( $\mathrm{Cy} 5 / \mathrm{Cy} 3$ ratios) of barcodes denoting the different strains in latrunculin-A treated and untreated conditions was assessed with the microarray chip. This experiment identified 51 phenotypic enhancements (Appendix 2). 25 of these were validated independently as individual strains to have increased sensitivity latrunculin-A and are listed in Table 4.1. These included deletion mutants of several genes involved in cytoskeletal organisation (ARP1, BBC1, BNR1, CAP1, CAP2, CIK1, KIP3, LDB18, and TPM1), tubulin complex (PAC10and YKE2), lipid metabolism (ERG2 andFEN1), and multi-drug resistance (PDR5 and SNQ2) (Fig. 4.4). The genes $B N R 1, C A P 1$, and $C A P 2$ are enriched for GO category "barbed-end actin filament capping" ( $\sim 99.4$ fold enrichment) and "negative regulation of actin filament depolymerisation" ( 99.4 fold enrichment). The genes ARP1, CIK1, KIP3 and LDB18 are enriched for GO category "establishment of mitotic spindle orientation and localisation" ( $\sim 53.0$ fold enrichment) and $B B C 1, B N R 1, C A P 1, C A P 2$, and TPM1 show

enrichment for the GO category "actin cytoskeleton organization" $(\sim 9.7$ fold enrichment).

Hypersensitive mutants were expected in these categories, because genes enriched for "actin cytoskeleton organisation" and "negative regulation of actin filament depolymerisation" protect against disruption of the actin cytoskeleton. Therefore, deletion mutants of these genes will be more sensitive to latrunculin-A, a compound 
that promotes actin cytoskeleton disassembly by binding and sequestering monomeric G-actin and preventing polymerisation of F-actin.

Interestingly, deletion mutants of genes associated with the microtubule cytoskeleton specifically, dynactin complex (ARP1 and LDB18), kinesin complex (CIK1 and KIP3) and tubulin complex assembly (PAC10 and YKE2) also demonstrated hypersensitivity to latrunculin-A. These were unexpected, as previous studies in both budding yeast and vertebrates have shown tubulin cytoskeleton to be unaffected by the latrunculin-A treatment $(121,122)$.

\begin{tabular}{|l|l|l|}
\hline ORF & Gene & Description \\
\hline YHR129C & ARP1 & Actin-related protein of the dynactin complex \\
\hline YJL020C & BBC1 & Protein possibly involved in assembly of actin patches \\
\hline YIL159W & BNR1 & Formin \\
\hline YKL007W & CAP1 & Alpha subunit of the capping protein (CP) heterodimer \\
\hline YIL034C & CAP2 & Beta subunit of the capping protein (CP) heterodimer \\
\hline YMR198W & CIK1 & Kinesin-associated protein \\
\hline YAL026C & DRS2 & Trans-Golgi network aminophospholipid translocase (flippase) \\
\hline YMR202W & ERG2 & C-8 sterol isomerase \\
\hline YCR034W & FEN1 & Fatty acid elongase \\
\hline YHL031C & GOS1 & v-SNARE protein involved in Golgi transport \\
\hline YGL168W & HUR1 & Protein of unknown function \\
\hline YGL216W & KIP3 & Kinesin-related motor protein involved in mitotic spindle positioning \\
\hline YLL049W & LDB18 & Component of the dynactin complex \\
\hline YGR078C & PAC10 & Part of the heteromeric co-chaperone GimC/prefoldin complex \\
\hline YOR153W & PDR5 & Plasma membrane ATP-binding cassette (ABC) transporter \\
\hline YDR011W & SNQ2 & Plasma membrane ATP-binding cassette (ABC) transporter \\
\hline YIL073C & SPO22 & Meiosis-specific protein essential for chromosome synapsis \\
\hline
\end{tabular}




\begin{tabular}{|l|l|l|}
\hline YER111C & SWI4 & DNA binding component of the SBF complex (Swi4p-Swi6p) \\
\hline YBL054W & TOD6 & PAC motif binding protein involved in rRNA and ribosome biogenesis \\
\hline YNL079C & TPM1 & Major isoform of tropomyosin \\
\hline YDR074W & TPS2 & Phosphatase subunit of the trehalose-6-phosphate synthase complex \\
\hline YDR247W & VHS1 & Cytoplasmic serine/threonine protein kinase \\
\hline YLR200W & YKE2 & Subunit of the heterohexameric Gim/prefoldin protein complex \\
\hline YBR178W & YBR178W & Dubious open reading frame unlikely to encode a functional protein \\
\hline YFR016C & YFR016C & Putative protein of unknown function \\
\hline
\end{tabular}

Table 4.1- List of genes deletion mutants hypersensitive to $28 \mathrm{nM}$ latrunculin-A in a barcode microarray experiment. 25 phenotypic enhancements were identified from a DNA barcode microarray using the haploid PDR-deficient mutants. Genes were annotated according to SGD (62). Highlighted in green are genes encoding for components of the cytoskeleton. 


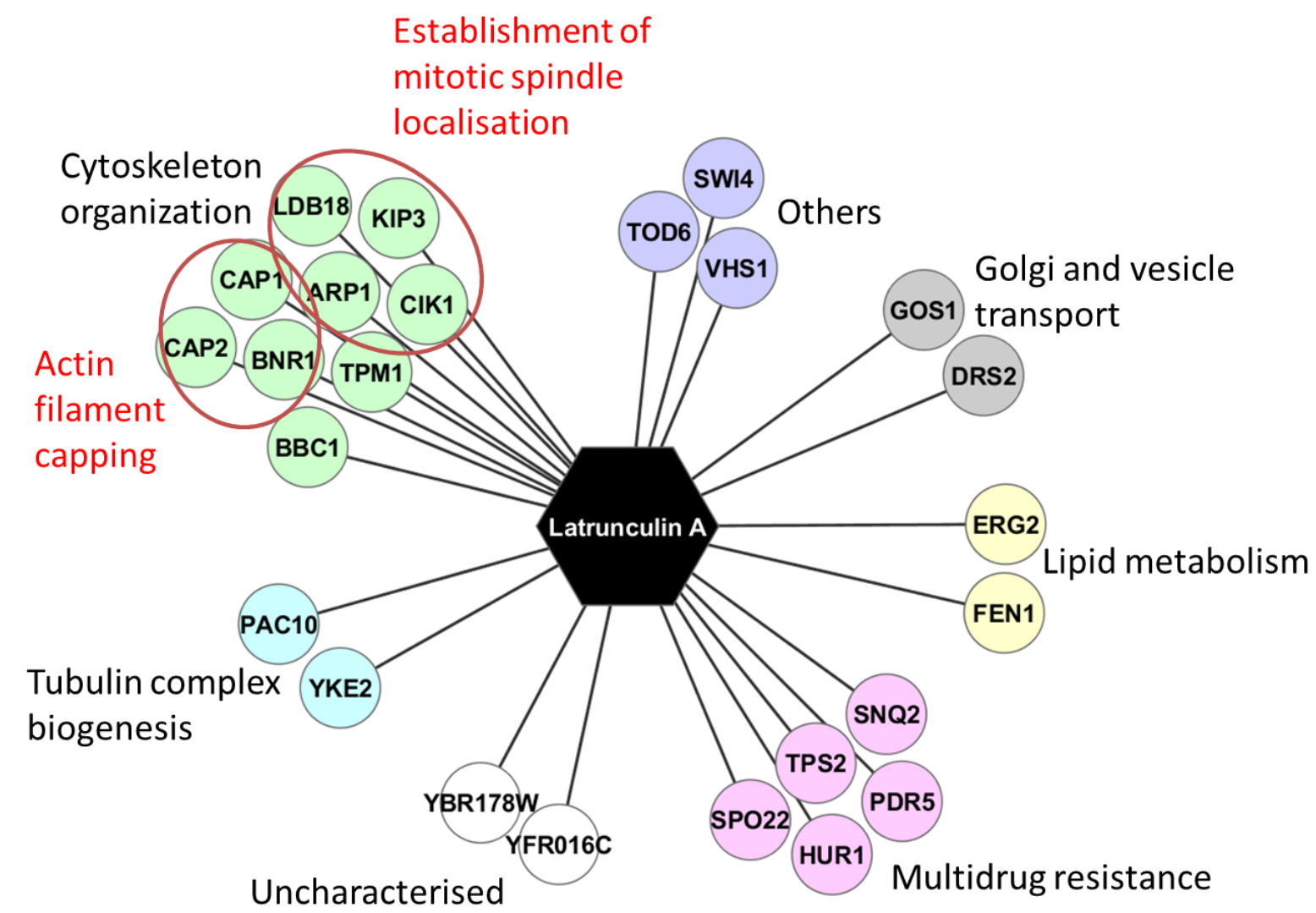

Figure 4.4 - Network diagram for genes hypersensitive to $28 \mathrm{nM}$ latrunculin-A. Genes were compiled into a network diagram using Cytoscape and assigned to GO terms based on statistical over-representation. Genes were enriched for biological processes including actin cytoskeletal organisation, barbed-end actin filament capping, and establishment of mitotic spindle localisation. 


\section{$\underline{\text { Discussion }}$}

\subsection{Latrunculin-A is a PDR5 substrate.}

Based on the dose response shown in Fig. 4.2, latrunculin-A was identified as a PDR substrate based on the increased sensitivity of the $\Delta p d r 1 \Delta p d r 3$ strain. This has not been previously shown. In the subsequent dose response assay (Fig. 4.3a), Pdr5p was identified as the major contributor for Pleiotropic Drug Resistance (PDR) against latrunculin-A. Furthermore, the loss of Yor1p conferred resistance to latrunculin-A (Fig. 4.3a-b). This is consistent with findings by Kolaczkowska et al. where inactivation of any of the major PDR transporters (PDR5, SNQ2 or YORI) lead to induction of the remaining paralogs (37) and the resistance observed was most likely due to upregulation Pdr5p in the absence of YOR1. However, this resistance was not observed with loss of SNQ2. Kolaczkowska et al. (2008) showed an induction of PDR5 in the $\Delta s n q 2$ mutant was present, but to a lesser degree than in $\Delta y o r 1$ mutant (37).

\subsection{Deletion mutants hypersensitive to latrunculin-A}

The sensitivity of gene deletion strains to latrunculin-A was assessed in a DNA barcode microarray experiment (Chapter 2 Materials and Methods) utilizing the haploid PDRdeficient mutants. 25 of the phenotypic enhancements were validated from the DNA barcode microarray experiment by increased sensitivity to latrunculin-A in a growth inhibitory assay (Chapter 2 Materials and Methods). Several of these mutants were indicative of the actin cytoskeleton disruption (Table 4.1). In addition, several other biological processes were also affected by latrunculin-A treatment, including tubulin 
complex assembly and mitotic spindle orientation and localisation. Out of the 25 phenotypic enhancements, deletion mutants of only five genes (ARP1, BBC1, BNR1, CAP1 and YFR016C) were among the top 120 genes (P value <0.001) previously identified by Hillenmeyer et al. using homozygous deletion mutants in a DNA barcode microarray against latrunculin-A (116). Of these, only $B B C 1, B N R 1$ and $C A P 1$ were among the actin associated genes, as described in SGD. In contrast, the haploid deletion mutants in the PDR-deficient background identified the above mutants and several additional cytoskeletal genes, including CAP2, CIK1, KIP3, LDB18, and TPM1, demonstrating the increased sensitivity afforded by the PD-DMA. This screen also identified several other genes involved in tubulin complex assembly and Golgi transport genes, amongst others, which were also validated to show hypersensitivity latrunculinA. These genes have not been previously identified in genome-wide chemical genetic screens employing latrunculin-A, and are genes that modify the main actin-associated pathways. The PD-DMA, thus, identified more cytoskeleton organisation genes and actin cytoskeleton genes than the conventional single deletion mutant array in the wildtype background, and did so at a lower drug concentrations, as latrunculin-A is a PDR substrate.

The proteins encoded by $C A P 1$ and $C A P 2$ form the F-actin capping complex which binds to the barbed end (fast growing end) of the actin filament and promotes actin polymerisation $(62,126)$. CAP proteins localise on actin patches and actin cables, and are required for stability of both (126). The gene $B N R 1$ encodes for a formin, which plays a role in nucleating actin to the barbed end of the filament (127). The major isoform of tropomyosin, TPM1 also binds and stabilises actin (126). Overall, the genes 
identified in this chapter stabilise and promote actin polymerisation and buffer the effect of latrunculin-A in terms of actin filament stabilisation.

Although the DNA barcode microarray experiment utilising the PDR-deficient mutants identified several actin cytoskeleton genes $(B B C 1, B N R 1, \mathrm{CAP} 1, C A P 2$, and TPM1) as buffering latrunculin-A, it also identified several genes involved in the tubulin cytoskeleton (ARP1, CIK1, KIP3, LDB18, PAC10and YKE2). These are involved in three distinct processes in the tubulin cytoskeleton: components of dynactin (ARPI and $L D B 18)$, kinesin associated microtubule motor activity (CIK1 and KIP3) and tubulin complex assembly (PAC10 and YKE2) (discussed in SGD). Two of the dynactin components, ARP1 and JNM1, were also among the cytoskeleton organisation genes identified by Hillenmeyer et al. (116). The published literature lacks these observations, presumably because the PDR-deficient strains were not used.

Recently, several microtubule and actin-associated proteins have been shown to involve multiple cytoskeleton-related pathways (123). The most well studied example of these are the plakins, which encode for large genes which can give splice variants with varying affinity for different cytoskeletons (123). As another example of cross talk between microtubule and microfilament cytoskeleton effects, the microtubuleassociated protein Sym1p, when overexpressed, can rescue defects of an actin-based motor, Myo2p (124). This suggests that latrunculin-A may affect proteins involved in the tubulin and actin cytoskeletons. The possibility that loss of Pdr1p and Pdr3p transcription factors may have led to the increase sensitivity of microtubule-associated genes to latrunculin-A seems unlikely, since several of the numerous genes identified here were also identified by Hillenmeyer et al. (116). 
There were a number of phenotypic enhancements that were not individually validated to demonstrate increased sensitivity to latrunculin-A using growth assays. The conditions used to validate the microarray results were not the same as those used in the microarray protocol. Hence, $100 \%$ correspondence between the two assays would not necessarily be expected. These deletion mutants may still demonstrate hypersensitivity to latrunculin-A under the conditions used for the microarray. Furthermore, not all the strains hypersensitive to latrunculin-A may have been identified in the DNA barcode microarray, because the latter requires genomic DNA extraction, PCR amplification and hybridisation to oligos on a microarray chip. That is an incremental number of assay steps during which the barcodes denoting the some deletion mutants may be lost. Furthermore, nearly $20 \%$ of the deletion mutants in the commercially available DMA carry point mutations in the barcodes and common priming sites (128) therefore, some barcodes may not hybridize, or hybridise with incorrect oligos on the microarray chip, although, using both UP and DN tags somewhat ameliorates this discrepancy.

In Tong et al., a systematic genetic analysis of the HOG pathway gene $N B P 2$ identified several actin and tubulin cytoskeleton-associated genes within the 27 phenotypic enhancements utilising $\triangle n b p 2$ as a query gene (11). Interestingly, this leaves two possibilities: firstly, that latrunculin-A may affect the microtubule-associated proteins through processes that utilise both actin and tubulin cytoskeletons. Alternatively latrunculin-A may have a target besides monomeric actin that may affect both actin and tubulin cytoskeletons. Either scenario is not suggested in the published model for latrunculin-A activity (122), where latrunculin-A is shown to bind monomeric actin in a 
1:1 ratio to form a complex and not interfere with microtubule assembly. Therefore, the results from the current study suggest, at the least, that latrunculin-A has a more intricate network effect, rather than simply having actin binding activity. 


\section{Identifying Molecular Mechanism of Plakortide-T Using the PD-DMA}

\section{$\underline{\text { Introduction }}$}

5.1.1 Plakortide-T, a novel marine natural product.

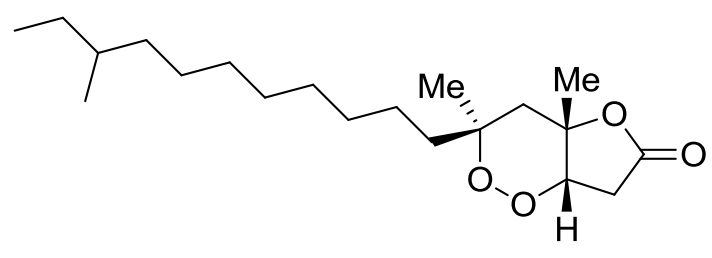

Figure 5.1 - Structure of plakortide-T

Plakortide- $\mathrm{T}$ is a novel cyclic peroxide isolated from Tongan marine sponges Plakinastrella and Plakortis spp. It belongs to a class of compounds comprising 1, 2dioxygenated five or six membered rings (129). These are of particular interest as they possess diverse pharmacological activity including antifungal, antibacterial, anti-tumour and anti-protozoal activity $(129,130)$. These cyclic peroxides have been particularly effective against murine leukaemia cell lines and human cancer cell lines (131). Particularly, plakortides F, I, K, L and Q are highly effective against the malaria and several Plakortis spp. metabolites are also effective against numerous other protozoans (reviewed in Dembitsky et al.) $(129,131)$.

Plakortide-T was employed in this chapter to further explore the utility of the PDRdeficient mutant array since plakortide-T was found to active in the PDR-deficient background and completely insensitive in wild-type yeast strains. Furthermore, the novel mode of action of other cyclic peroxides from Plakortis spp. mentioned below 
and their diverse activity against numerous human parasites and antitumor properties makes it an attractive compound to study.

\subsubsection{Proposed mechanism of action for Plakortis metabolites}

The mechanism underlying these diverse ranges of activities of these cyclic peroxides are yet to be elucidated. However, several Plakortis spp. metabolites promote the uptake of $\mathrm{Ca}^{2+}$ by the sarcoplasmic reticulum in cardiac tissue via a $\mathrm{Ca}^{2+}$ ATPase (132). Particularly, plakortide $\mathrm{F}$ acid (PFA) has been shown to disrupt $\mathrm{Ca}^{2+}$ homeostasis in yeast (129). The calcium homeostasis disruption by PFA was illustrated in several experiments. The expression profile for PFA was similar with high exposure to $\mathrm{CaCl}_{2}$ and to a known calcium homeostasis disrupting agent, amiodarone (129). Several of the deletion mutants of calcineurin subunits i.e. $\Delta c n a 1, \Delta c n b 1, \Delta c m p 2$ and calcineurin activated gene $\Delta c r z 1$ and $\mathrm{Ca}^{2+}$ transporters i.e. $\Delta p m r 1, \Delta p m c 1, \Delta m i d 1$ and $\Delta c c h 1$ demonstrated hypersensitivity to PFA and the activity of PFA is enhanced by calcineurin inhibitors FK506 and cyclosporine-A (129). In addition, the calcineurin dependent response element driving lacZ fusion, $4 x C D R E:: l a c Z$ was highly induced by PFA treatment. Moreover, an elemental analysis of PFA treated cells showed a $\sim 7$ fold increase in intra cellular calcium and a $\sim 2$ fold increase in zinc while no change in manganese, iron, aluminium, Sodium, Magnesium and potassium was observed (129).

\subsection{Calcium homeostasis in yeast}

Calcium is used as a second messenger in response to numerous stress conditions, including hypertonic shock, hyperosmotic shock, cold shock, aluminium toxicity, 
arsenic stress, magnesium starvation, high $\mathrm{pH}$, and in response to generation of reactive oxygen species (133-135). The $\mathrm{Ca}^{2+}$ levels are normally kept at very low levels (10-100 $\mathrm{nM})$ with in the cytosol (136). These processes lead to a transient increase in cytosolic $\mathrm{Ca}^{2+}$ levels which can be mediated either by an influx of $\mathrm{Ca}^{2+}$ via the plasma membrane transporters, Cch1p and Mid1p, or release of calcium from intercellular stores such as the vacuole and ER via Yvc1p (134). The release of calcium from intracellular stores can promote the influx of calcium from the extra cellular environment via Mid1p and Cch1p plasma membrane transporters in a process known as 'capacitive calcium entry' and conversely, the influx of $\mathrm{Ca}^{2+}$ from the extra cellular environment also stimulates the release of $\mathrm{Ca}^{2+}$ from the intracellular stores via Yvc1p in a process known as 'calcium induced calcium release' (134).

The cytosolic $\mathrm{Ca}^{2+}$ levels are restored to normal very low levels by the vacuolar transporters, Pmc1p and Vcxlp and the ER/Golgi transporter Pmrlp which pumps cytosolic $\mathrm{Ca}^{2+}$ into the vacuole and the ER/Golgi respectively (134). This response is mediated by calmodulin which is activated by binding of cytosolic $\mathrm{Ca}^{2+}$ during the transient increase (134). $\mathrm{Ca}^{2+} /$ calmodulin binds and activates calcineurin, a multiprotein complex encoded by two catalytic subunits (Cna1p and Cmp2p/Cna2p), and a regulatory subunits (Cnb1p) (137). The activated calcineurin subsequently dephosphorylates and activates a transcription factor, Crz1p, which translocates to the nucleus and binds CDRE (crz1-resposive elements) on target promoters to induce the expression of several genes including PMRI and PMC1 (135). These latter genes are required to restore basal level of cytosolic $\mathrm{Ca}^{2+}$. 


\subsection{Disruption of $\mathrm{Ca}^{2+}$ homeostasis by reactive oxygen species (ROS)}

The excessive and uncontrolled increased cytosolic $\mathrm{Ca}^{2+}$ concentration has shown to induce apoptosis in other model systems. In mammalian cell lines $\mathrm{Ca}^{2+}$ overloading leads to mitochondrial dysfunction and ROS generation and apoptosis follows as a consequence (134). Several compounds that cause an increase in cytosolic $\mathrm{Ca}^{2+}$ also generates reactive oxygen species (138). A prime example of such is $\mathrm{H}_{2} \mathrm{O}_{2}$ and t-butyl hydroperoxide $(t \mathrm{BOOH})$ which causes an increase in cytosolic $\mathrm{Ca}^{2+}$ contributed mostly by the release of $\mathrm{Ca}^{2+}$ from internal calcium stores (134). The concentration of the cytosolic $\mathrm{Ca}^{2+}$ achieved during treatment with compound appears to be inversely proportional to the amount of resistance to that compound (134). The mutant $\Delta y v c l$ has a reduced levels of cytosolic $\mathrm{Ca}^{2+}$ in response to $\mathrm{H}_{2} \mathrm{O}_{2}$ treatment and it shows greater tolerance to $\mathrm{H}_{2} \mathrm{O}_{2}$ whereas $\Delta$ midl mutant with greater levels of cytosolic $\mathrm{Ca}^{2+}$ in response to $\mathrm{H}_{2} \mathrm{O}_{2}$ treatment shows less tolerance to $\mathrm{H}_{2} \mathrm{O}_{2}$ (134). This is also consistent with $\mathrm{Al}^{3+}$ toxicity where $\Delta p m c 1$ mutant with increased cytosolic $\mathrm{Ca}^{2+}$ than wild type demonstrates reduced $\mathrm{Al}^{3+}$ tolerance (133).

Apoptosis in yeast can be induced by exposure to a myriad of conditions including high salt $(\mathrm{NaCl})$ stress, acetic acid, $\mathrm{HOCl}$, heat stress, UV radiation, high levels of $\mathrm{Al}, \mathrm{Cd}$, $\mathrm{Cu}, \mathrm{Mg}, \mathrm{Fe}$ and even glucose which in the absence of other nutrients generates reactive oxygen species $(138,139)$. The ROS generated include both oxidants and reductants, collectively known as ROS, which can cause havoc within cells where they cause damage to a wide range of molecules including amino acids, proteins, lipids, and nucleic acids (138). One of the main targets of ROS is the oxidation of free thiol groups on proteins to form disulphides, sulphenic and sulphinic acids (138). In addition 
to the generation of primary ROS such as $\mathrm{H}_{2} \mathrm{O}_{2}$, primary ROS in turn can react with other molecules within the cell to generate other reactive species (138). Therefore, the generally used term "oxidative stress" refers to various conditions specific to the compound generating the ROS. This was illustrated by Jamieson et al. where different genes sets were activated in response to treatment with $\mathrm{H}_{2} \mathrm{O}_{2}$ or menadione (140).

Changes in subcellular localisation of Yap1p, the oxidative stress response regulator, in response to the oxidative environments has been studied in depth. Under normal conditions Yap1p is held in the cytoplasm by its interaction with Crm1p which acts as a nuclear export factor for Yap1p (141). However, this interaction is highly sensitive to oxidative damage (141), in oxidative environments Yap1p is oxidised by glutathione peroxide and is translocated into the nucleus by a process which requires Ypb1p (138, 142). The nuclear localisation of Yap1p in oxidative environments was utilised in this chapter to determine the activation of the oxidative stress response. 


\section{Materials and methods}

\subsubsection{Yeast strains}

All $S$. cerevisiae strains used in this chapter were derived from the diploid strain BY4743 (54) and were constructed by PCR mediated disruption (see query strain construction) and are as follows:

\begin{tabular}{|c|c|}
\hline Strain & Genotype \\
\hline BY4743 & $\begin{array}{l}\text { MATa/a his3 } \Delta 1 / \text { his3 } \Delta 1 \text { leu2 } \Delta 0 / \text { leu2 } \Delta O \text { LYS2/lys2 } \Delta 0 \text { met15 } \Delta 0 / M E T 15 \\
\text { ura3 } \Delta 0 / \text { ura3 } \Delta 0\end{array}$ \\
\hline Y7092 (wild-type) & 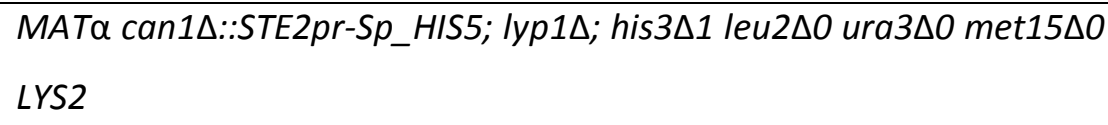 \\
\hline $\begin{array}{l}\Delta p d r 1 \Delta p d r 3 \text { (PDR-deficient } \\
\text { strain, Y7092 background) }\end{array}$ & 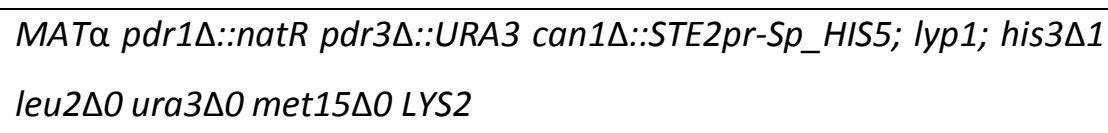 \\
\hline$\Delta x x x \Delta p d r 1 \Delta p d r 3$ & 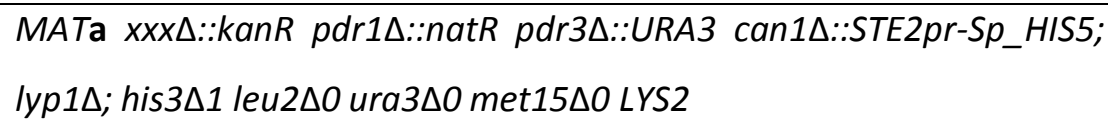 \\
\hline Y8205 & 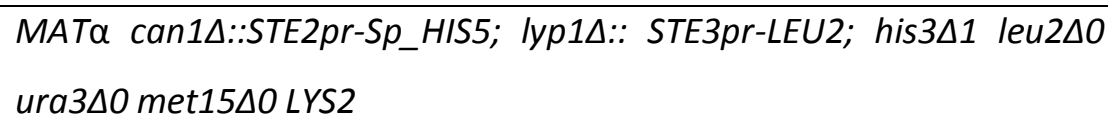 \\
\hline $\begin{array}{l}\Delta p d r 1 \Delta p d r 3 \text { (Y8205 } \\
\text { derived and utilised only in } \\
\text { the construction of PDR- } \\
\text { deficient GFP reporter } \\
\text { strains) }\end{array}$ & 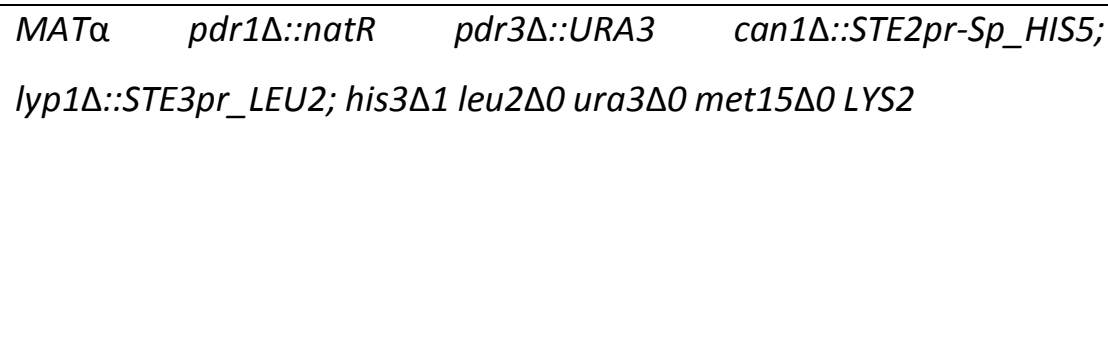 \\
\hline CRZ1-GFP & 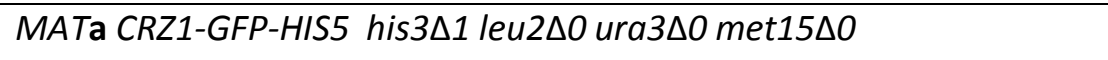 \\
\hline HOG1-GFP & 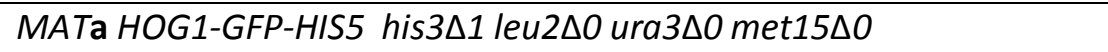 \\
\hline MSN2-GFP & 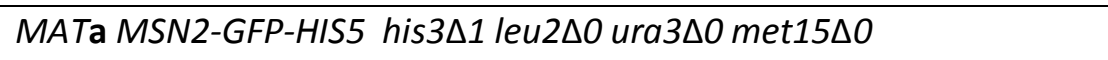 \\
\hline ZAP1-GFP & 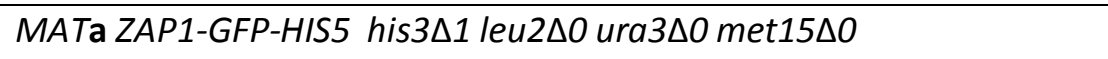 \\
\hline ZRC1-GFP & 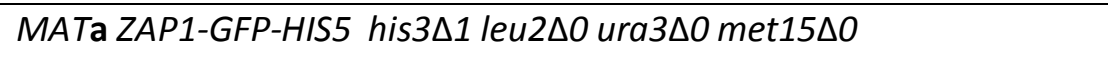 \\
\hline COT1-GFP & MATa COT1-GFP-HIS5 his3 $\Delta 1$ leu2 $\Delta O$ ura3 $\Delta O$ met $15 \Delta O$ \\
\hline CRZ1-GFP $\triangle p d r 1 \Delta p d r 3$ & 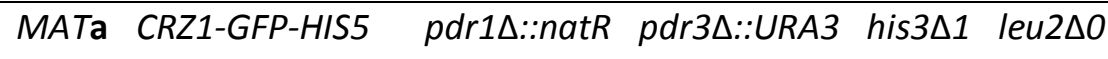 \\
\hline
\end{tabular}




\begin{tabular}{|c|c|}
\hline (CRZ1-GFP Derived) & ra3 $\Delta 0$ met15 $\Delta 0$ \\
\hline $\begin{array}{l}\triangle x x x \quad \Delta p d r 1 \Delta p d r 3 \quad C R Z 1- \\
\text { GFP } \\
\text { (Y8205 derived) }\end{array}$ & 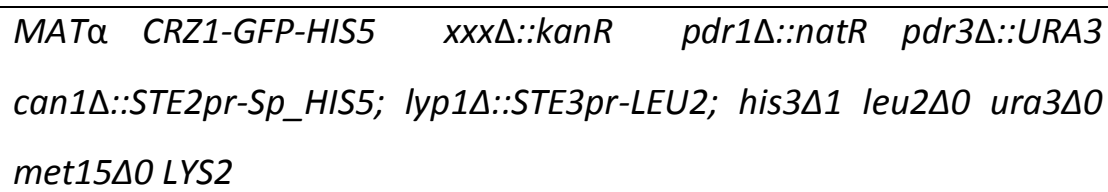 \\
\hline $\begin{array}{l}\text { YAP1-GFP } \triangle p d r 1 \Delta p d r 3 \\
\text { (Y8205 derived) }\end{array}$ & 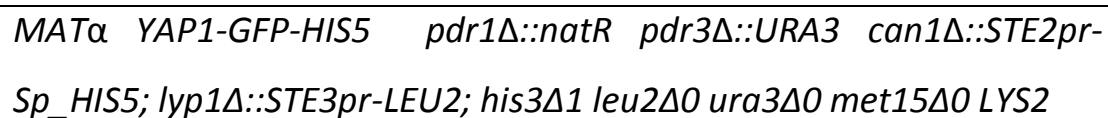 \\
\hline
\end{tabular}

Table 5.1 - Yeast strains used in this chapter. The yeast strains were constructed either by PCR mediate disruption or mating followed by random spore analysis according to chapter 2 (Materials and Methods).

\subsubsection{Growth media and components}

All S. cerevisiae and E. coli strains were cultured in growth media as stated in Chapter 2 (Materials and Methods).

\subsubsection{Compounds and chemicals}

Compounds: latrunculin-A and plakortide-T (kindly provided by Peter Northcote, School of Chemical and Physical Sciences, Victoria University of Wellington) was dissolved in DMSO at a concentration of $10 \mathrm{mM}$ and stored at $-20^{\circ} \mathrm{C}$.

Dithiothreitol (DTT) (Sigma-Aldrich) and tunicamycin (Sigma-Aldrich) was dissolved in $\mathrm{ddH}_{2} \mathrm{O}$ at the concentration of $1 \mathrm{mM}$ and was stored at $-20^{\circ} \mathrm{C}$. Hydrogen peroxide $\left(\mathrm{H}_{2} \mathrm{O}_{2}\right)$ (Sigma-Aldrich) was dissolved in $\mathrm{ddH}_{2} \mathrm{O}$ at a concentration of $10 \mathrm{mM}$ was stored at $4{ }^{\circ} \mathrm{C}$. Calcium chloride (Sigma-Aldrich) and ninc chloride (Sigma-Aldrich) was dissolved in $\mathrm{dH}_{2} \mathrm{O}$ at $1 \mathrm{M}$ and stored at room temperature. 
Menadione was dissolved in ethanol at the concentration of $100 \mathrm{mM}$ and was stored at room temperature.

\subsubsection{Dose response assays.}

Dose response assays were performed as described in Chapter 2 (Materials and Methods).

\section{$\underline{5.4 .5}$ Cytotoxicity assay}

A half $\log$ dose response assay was performed on $\Delta p d r l \Delta p d r 3$ strain employing plakortide-T as described in Chapter 2 (Materials and Methods). At $1 \mathrm{~h}$ intervals $1 \mu \mathrm{L}$ volumes of each culture was spotted onto a SC plate and were incubated for $48 \mathrm{~h}$. The cells were examined for recover after incubation by visual inspection of the spots for colony formation.

\subsubsection{DNA barcode microarray}

The DNA barcode microarray was performed on haploid PDR-deficient gene deletion pools employing plakortide-T as described in Chapter 2 (Materials and Methods).

\subsubsection{Reactive oxygen species generation assay}

$5 \times 10^{7} \Delta p d r 1 \Delta p d r 3$ cells in a volume of $50 \mu \mathrm{L}$ were treated with either plakortide-T, menadione or DMSO for $2 \mathrm{~h}$ at $30^{\circ} \mathrm{C}$. Following incubation, $50 \mu \mathrm{L}$ of $20 \%$ TCA was 
added and the sample was centrifuged at $13,000 \mathrm{rpm}$ for $10 \mathrm{~min}$. A master mix was made by the addition of the reagents listed in Table 5.1 below. $60 \mu \mathrm{L}$ of this master mix was transferred in to the wells of a 96 well culture plate along with $40 \mu \mathrm{L}$ of the TCAtreated cell supernatant prepared above. The OD of the mixture in each well was measured at $412 \mathrm{~nm}$ using the EnSpire Multimode Plate Reader Label-free System (Perkin-Elmer, Turku, Finland).

\begin{tabular}{|l|l|}
\hline Regents & Volumes $(\mu \mathrm{L})$ \\
\hline DTNB (10mM in EtOH) & 13 \\
\hline Tris- $\mathrm{HCl}$ (1 M buffered to PH 8.9) & 230 \\
\hline dd $_{2} \mathrm{O}$ & 600 \\
\hline Total volume & 1000 \\
\hline
\end{tabular}

Table 5.2 - Master mix for the DTNB assay

\subsubsection{Yap1p subcellular localisation assay}

The yeast strain, YAP1-GFP $\triangle p d r 1 \Delta p d r 3$ was assayed using fluorescent confocal microscopy in the presence of either plakortide- $\mathrm{T}, \mathrm{H}_{2} \mathrm{O}_{2}$, menadione or DMSO. Image acquisition was performed immediately following compound treatment as described in in Chapter 2 (Materials and Methods). Cells were visually identified and cytoplasmic to nuclear localisation of the Yap1p-GFP fusion protein was detected by visual inspection. 


\subsubsection{Subcellular localisation assays}

The appropriate GFP reporter strains were used in fluorescent confocal microscopy in the presence of either plakortide- $\mathrm{T}, \mathrm{ZnCl}_{2}, \mathrm{CaCl}_{2}$ or DMSO. The image acquisition was performed as described in Chapter 2 (Materials and Methods). Cells were visually identified and subcellular localisation of the GFP fusion protein was detected by visual inspection.

\section{$\underline{\text { 5.4.10 Zrc1p induction assay }}$}

ZRC1-GFP strain was used in confocal microscopy in the presence of appropriate concentrations $\mathrm{ZnCl}_{2}$ and $\mathrm{CaCl}_{2}$. The image acquisition was performed as described in Chapter 2 (Materials and Methods). Cells were identified and the induction of Zrc1pGFP was detected using Acapella, high-content imaging and analysis software (Perkin Elmer).

\section{$\underline{\text { 5.4.11 Calcium orange assay }}$}

$5 \times 10^{5} \Delta p d r 1 \Delta p d r 3$ cells in a volume of $100 \mu \mathrm{L}$ were pre-treated with $10 \mu \mathrm{M} \mathrm{Ca}^{2+}$ for 3 min before treating with $10 \mu \mathrm{M}$ calcium orange (dissolved in DMSO). The excitation and emission of the sample was measured at 549/576 nM using the EnSpire Multimode Plate Reader Label-free System (Perkin-Elmer). 
The $\Delta p d r 1 \Delta p d r 3$ were pre-treated with $\sim \mathrm{IC}_{30}$ of $25 \mu \mathrm{M}$ EGTA, a calcium ion chelator, for $15 \mathrm{~min}$ before being used in a dose response assays employing plakortide-T. The dose response assay was performed in Chapter 2 (Materials and Methods). Growth was determined by measuring the culture OD, $17 \mathrm{~h}$ after incubation. 


\section{$\underline{\text { Results }}$}

5.5 PDR-deficient strains are more sensitive to plakortide-T.

The biological activity of plakortide-T was assessed against the PD-DMA and wildtype background DMA. The pools of DMA $(\Delta x x x)$ and PD-DMA $(\Delta p d r 1 \Delta p d r 3 \Delta x x x)$ were used in dose response assay employing plakortide-T. Growth was determined by measuring by measuring culture OD of $\Delta x x x$ mutants and $\Delta p d r 1 \Delta p d r 3 \Delta x x x$ mutants after treatment with plakortide-T (Fig. 5.2). The $\Delta x x x$ mutants were insensitive to plakortide-T even at $100 \mu \mathrm{M}$, the highest concentration of plakortide-T tested in the dose response assay. In contrast, the $\Delta p d r 1 \Delta p d r 3 \Delta x x x$ mutants were sensitive to plakortide-T at low at nanomolar ranges with an $\mathrm{IC}_{50}$ of $\sim 703 \mathrm{nM}$. This result demonstrates plakortide-T as a PDR substrate. However, complete inhibition was not observed with plakortide-T. 


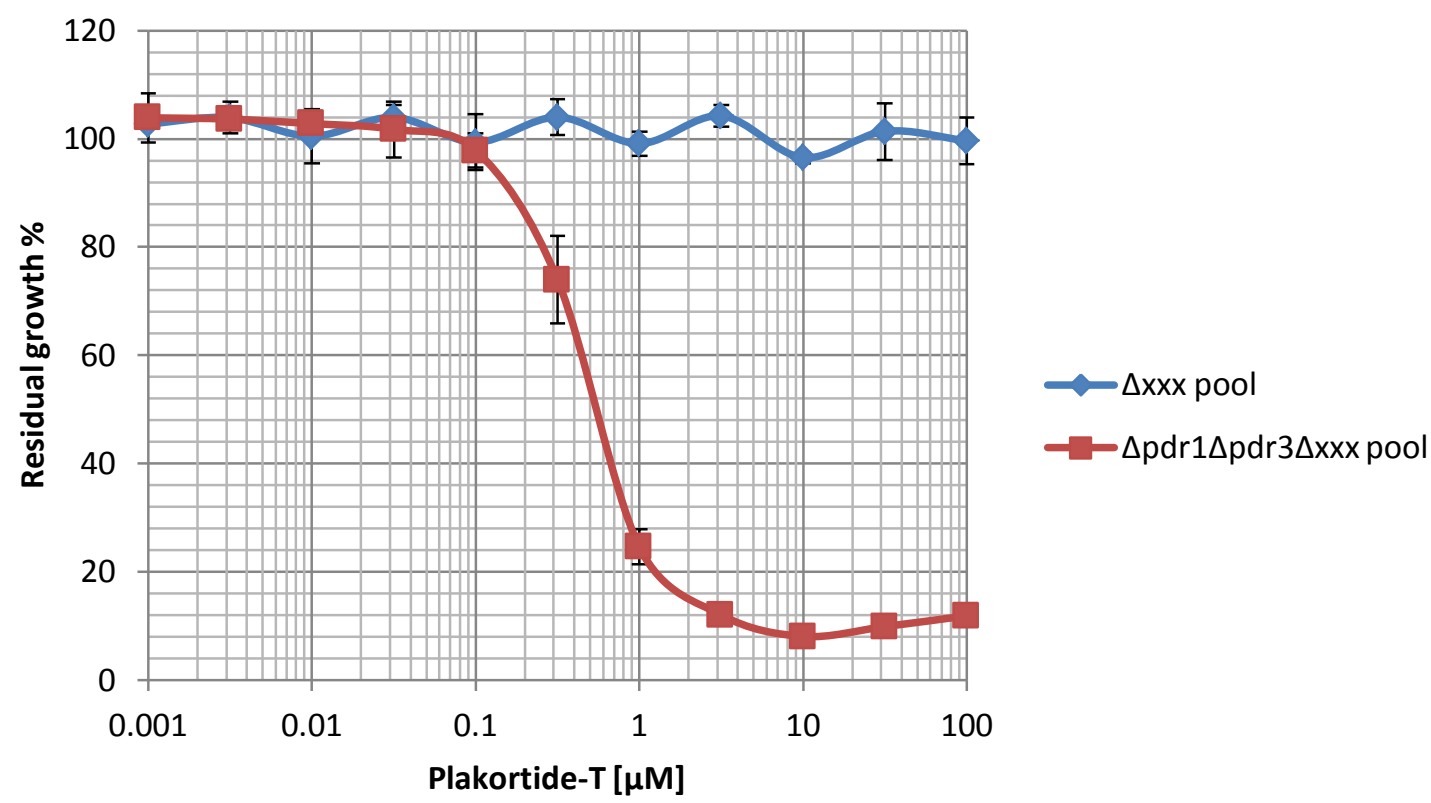

Figure 5.2- The PD-DMA strains are sensitive to Plakortide-T and not the DMA. In a dose response assay DMA $(\Delta x x x)$ and PD-DMA $(\Delta p d r 1 \Delta p d r 3 \Delta \mathrm{xxx})$ pools were assessed in their sensitivity to plakortide-T. The PD-DMA strains were sensitive to plakortide-T, while the DMA strains in the wild-type background were completely resistant to plakortide-T.

To investigate the lack of complete inhibition further, the wild-type yeast strain and the PDR-deficient strain, $\Delta p d r 1 \Delta p d r 3$ were used in a dose response assay employing plakortide-T (Fig.5.3). The sensitivities of the $\Delta p d r 1 \Delta p d r 3$ strain and the wild-type strain to plakortide-T were similar to what was observed with the two deletion mutant pools. The wild-type yeast strain was resistant to plakortide-T at all the concentration tested, while the PDR-deficient strain was sensitive to plakortide- $\mathrm{T}$ with an $\mathrm{IC}_{50} \sim 821$ nM. Approximately $11 \%$ residual growth was observed for the PDR-deficient strain at $10 \mu \mathrm{M}$ plakortide-T. Following this dose response assay it was noted that plakortide-T was heat labile. This would have resulted from opening of the cyclic peroxide ring responsible for the compounds bioactivity to a diol which was biologically inactive. 


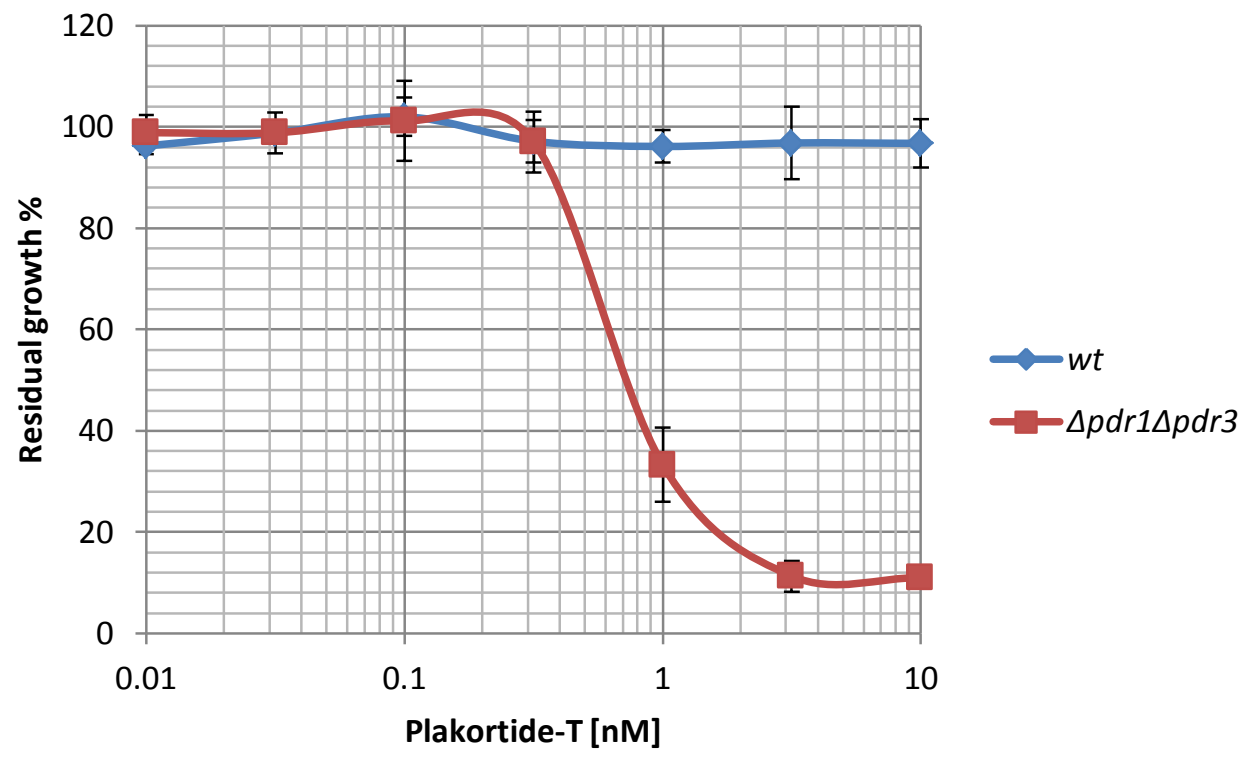

Figure 5.3- The PDR-deficient strain are sensitive to Plakortide-T and not the wild-type. In a dose response assay PDR-deficient strain, $\Delta p d r 1 \Delta p d r 3$ and the wild-type yeast strain were assessed for their sensitivity to plakortide-T. The PDR-deficient strain was sensitive to plakortide-T, while the wild-type strain was completely resistant to plakortide-T. Complete inhibition of the PDR-deficient strain was not observed for plakortide-T.

\section{$\underline{5.6 \text { Plakortide-T is cytostatic. }}$}

The recovery of the $\Delta p d r l \Delta p d r 3$ cells from plakortide-T treatment was assessed in a cytotoxicity assay by spotting cells on to fresh SC media (Fig. 5.4). In the cytotoxicity assay $\Delta p d r 1 \Delta p d r 3$ mutants recovered from plakortide- $\mathrm{T}$ treatment at all the concentration and treatment duration tested. This shows that plakortide-T reversibly inhibits the growth of $\Delta p d r 1 \Delta p d r 3$ cells, and therefore is cytostatic in its mechanism. 


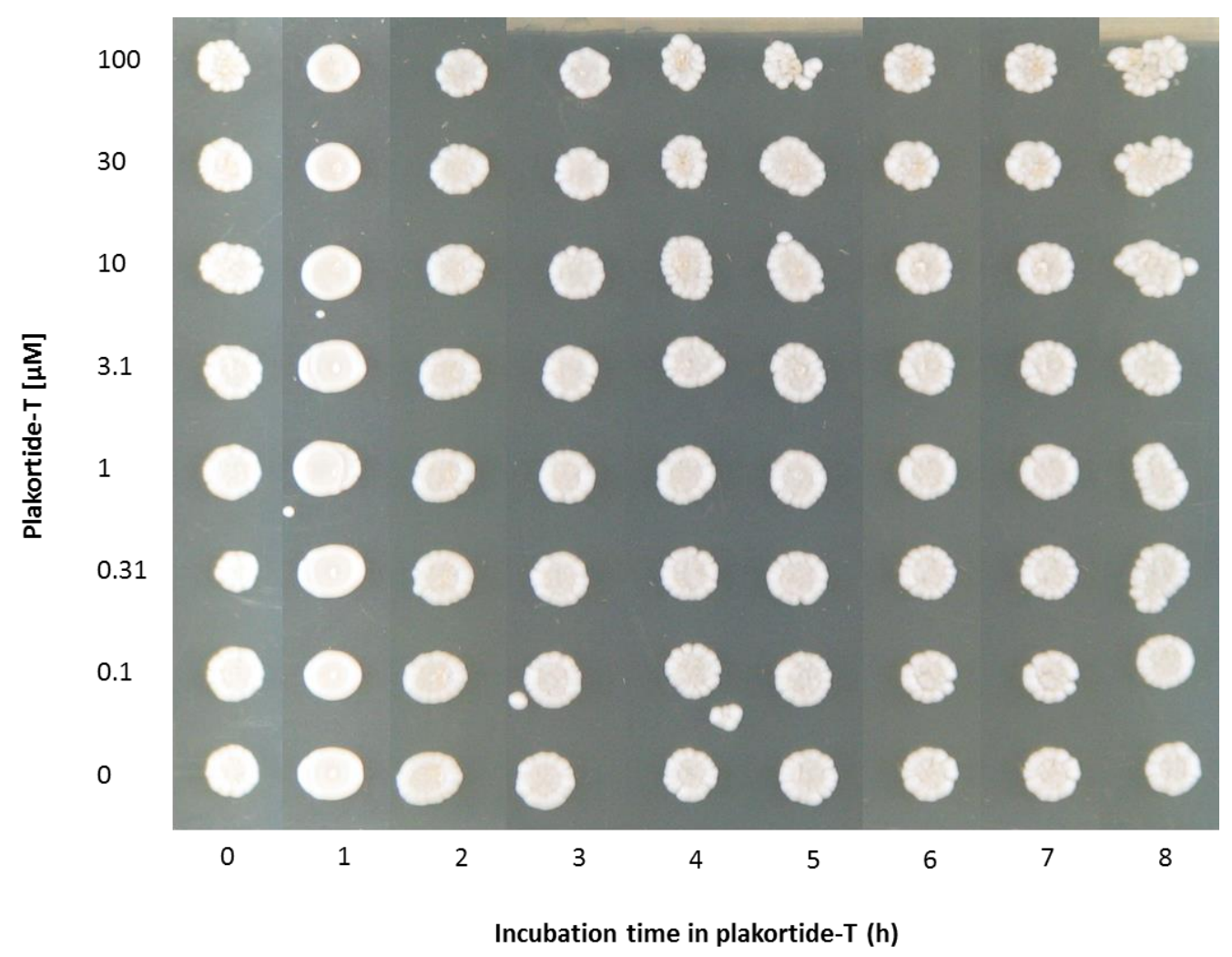

Figure 5.4- Plakortide-T cytotoxicity assay. A dose response assay was performed on PDRdeficient strain, $\Delta p d r \Delta 1 p d r 3$, employing plakortide-T. At $1 \mathrm{~h}$ intervals, $1 \mu \mathrm{L}$ volumes of the culture was spotted onto a SC plate from each of the concentrations of plakortide-T and were incubated at $30^{\circ} \mathrm{C}$ for $48 \mathrm{~h}$. Cells treated at all the plakortide-T concentrations recovered and ability of the cells to grow were unaffected by the length of incubation in plakortide-T. The images of the plates were cropped using power point to construct the above image.

\subsection{Plakortide-T is not a substrate for the major PDR efflux transporters}

The deletion mutants of the major PDR drug efflux transporter $(\Delta p d r 5, \Delta s n q 2, \Delta y o r 1)$ and several other minor PDR pump mutants ( $\Delta p d r 10, \Delta p d r 11, \Delta p d r 12$ and $\Delta p d r 15)$ were used in a dose response assay employing the PDR substrate plakortide-T. Growth was determined by measuring OD of the culture after treatment with plakortide-T and was compared against growth of both the $\Delta$ his 3 control strain and the PDR-deficient strain, $\Delta p d r 1 \Delta p d r 3$ (Fig. 5.5). The $\Delta p d r 1 \Delta p d r 3$ strain was sensitive to plakortide-T as 
expected and showed an $\mathrm{IC}_{50} \sim 6.30 \mu \mathrm{M}$. The sensitivities of PDR drug efflux transporter mutants, $\Delta p d r 5, \Delta s n q 2, \Delta y o r 1, \Delta p d r 10, \Delta p d r 11, \Delta p d r 12$ and $\Delta p d r 15$ were not significantly different from the $\Delta h i s 3$ control and were insensitive at all the concentrations of plakortide-T used in the dose response assay. This suggests that resistance to plakortide-T in wild-type yeast do not arise solely through one of the major or the minor drug efflux transporters screened in the dose response assay.

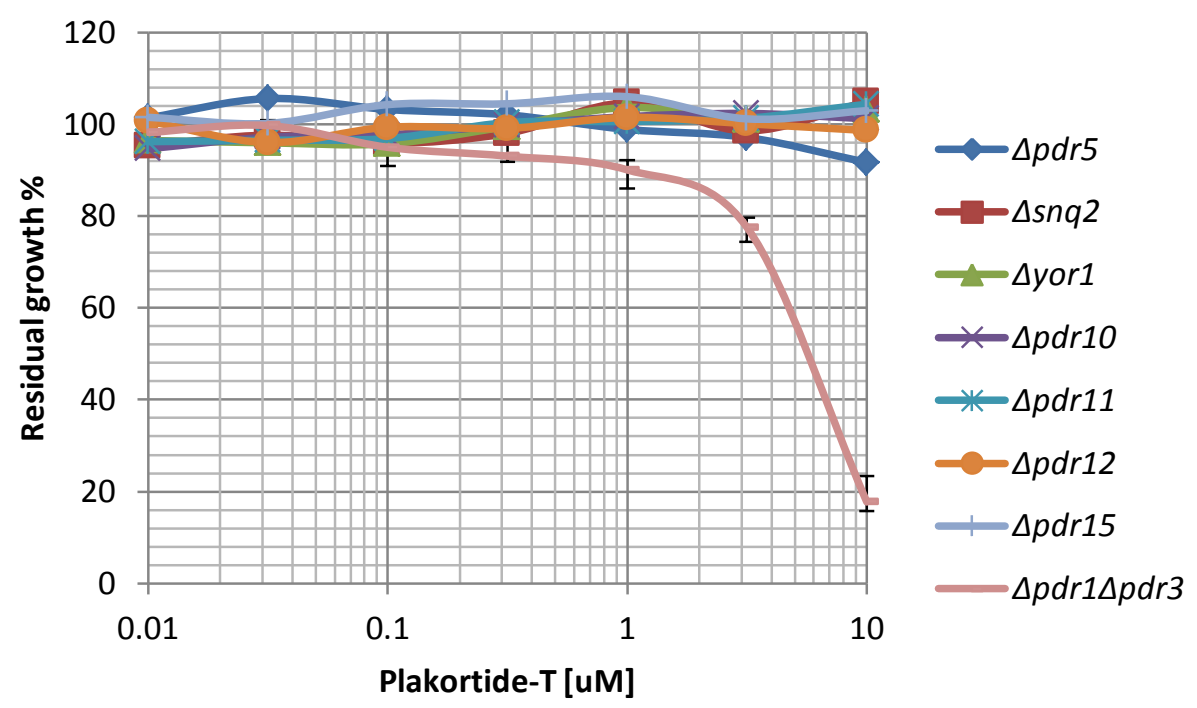

Figure 5.5 - The major drug efflux transporters do not individually contribute to plakortide-T resistance. Dose response assays were performed on the PDR pump mutant strains; $\Delta p d r 5$, $\Delta s n q 2, \Delta y o r 1, \Delta p d r 10, \Delta p d r 11, \Delta p d r 12$ and $\Delta p d r 15$ and the control strains; $\Delta h i s 3$ and $\Delta p d r 1 \Delta p d r 3$ against the PDR substrate plakortide-T. The $\Delta p d r 1 \Delta p d r 3$ was the most sensitive to plakortide-T. The PDR pump mutants; $\Delta p d r 5, \Delta s n q 2, \Delta y o r 1, \Delta p d r 10, \Delta p d r 11, \Delta p d r 12$ and $\Delta p d r 15$ and the $\Delta h i s 3$ control strain had similar sensitivities. 
5.8 Addition of either $\mathrm{Ca}^{2+}$ or $\mathrm{Zn}^{2+}$ increases the potency of plakortide- $\mathrm{T}$ in a synergistic manner.

Increased intracellular $\mathrm{Ca}^{2+}$ and $\mathrm{Zn}^{2+}$ in the elemental analysis of PFA treated cells suggested a possible role of $\mathrm{Ca}^{2+}$ and $\mathrm{Zn}^{2+}$ homeostasis disruption in the mechanism of action of cyclic peroxides. $\mathrm{Ca}^{2+}$ and $\mathrm{Zn}^{2+}$ homeostasis disruption was assessed using the PDR-deficient strain, $\Delta p d r l \Delta p d r 3$ in dose response assays in employing plakortide-T, with either $300 \mathrm{mM} \mathrm{CaCl}{ }_{2}\left(\sim \mathrm{IC}_{30}\right)$ or $2.5 \mathrm{mM} \mathrm{ZnCl}_{2}\left(\sim \mathrm{IC}_{30}\right)$. The growth was determined by measuring the culture OD and was compared against the sensitivity of the PDR-deficient strain treated with plakortide-T alone (Fig. 5.6). The PDR-deficient strain treated with only plakortide- $\mathrm{T}$ had an $\mathrm{IC}_{50}$ of $\sim 4.7 \mu \mathrm{M}$. Addition of either $\mathrm{ZnCl}_{2}$ or $\mathrm{CaCl}_{2}$ with plakortide- $\mathrm{T}$ increased the sensitivities of the PDR-deficient strain in comparison to plakortide-T alone. These increased sensitivities were synergistically greater than additive effect of either $\mathrm{ZnCl}_{2}$ or $\mathrm{CaCl}_{2}$ with plakortide-T. The strongest synergy was observed with $\mathrm{CaCl}_{2}\left(\mathrm{IC}_{50} \sim 1.6 \mu \mathrm{M}\right)$ followed by $\mathrm{ZnCl}_{2}\left(\mathrm{IC}_{50} \sim 3.0 \mu \mathrm{M}\right)$. These suggest that increased levels of calcium and zinc can contribute towards the cytotoxic mechanism of plakortide-T.

At the highest concentration of plakortide- $\mathrm{T}$ with $\mathrm{ZnCl}_{2}$, a slightly higher residual growth was observed in comparison to treatment with plakortide-T alone. This was not observed when the experiment was repeated only for this concentration (results not shown). 


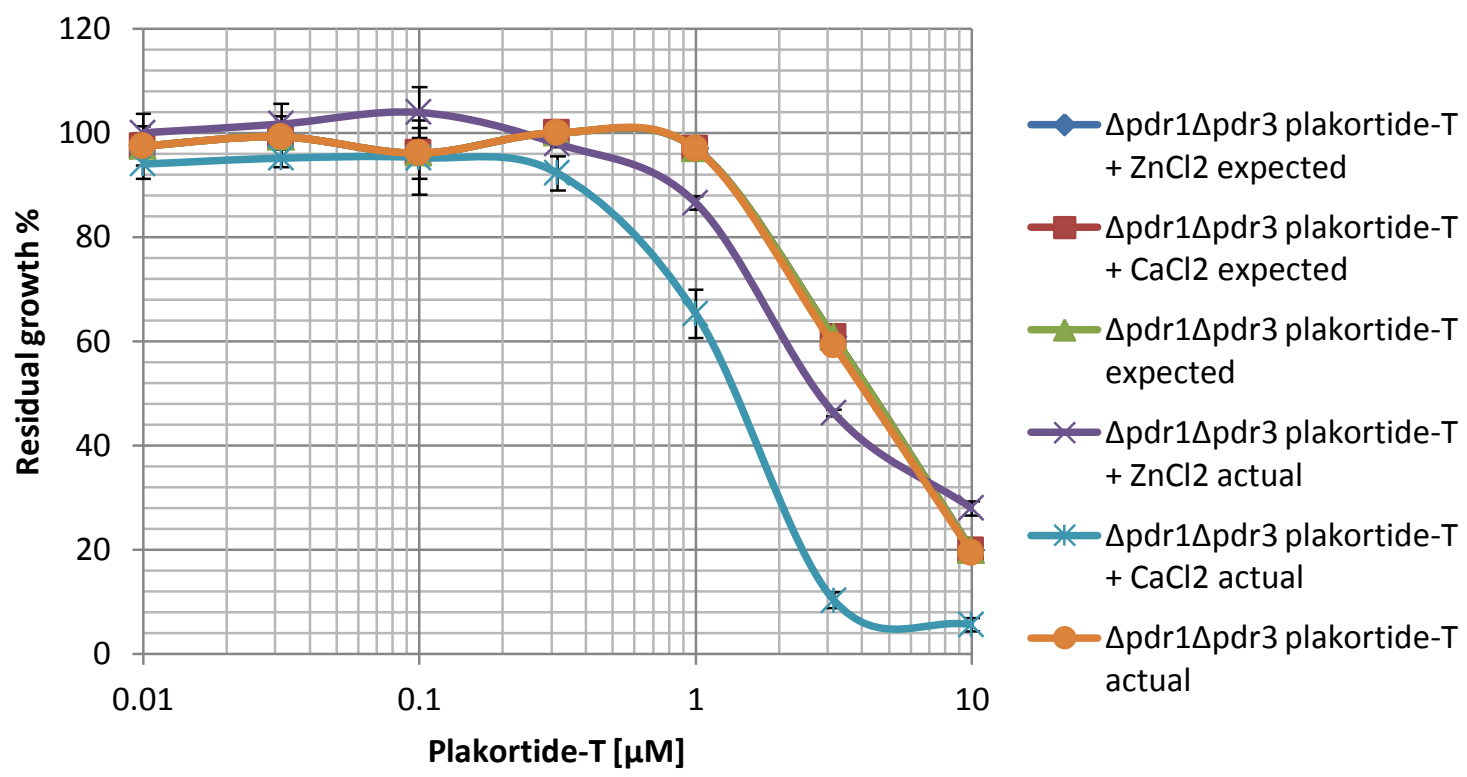

Figure 5.6 $-\mathrm{Ca}^{2+}$ and $\mathrm{Zn}^{2+}$ increases the potency of Plakortide-T in a synergistic manner. Dose response assays were performed on PDR-deficient strain, $\Delta p d r 1 \Delta p d r 3$ against, plakortide-T and $2.5 \mathrm{mM} \mathrm{ZnCl}_{2}$, plakortide-T and $300 \mathrm{mM} \mathrm{CaCl}_{2}$, and plakortide-T alone. Addition of sublethal concentration of $\mathrm{ZnCl}_{2}$ enhances the potency of plakortide-T in synergistic manner. Similarly, addition of sub-lethal concentration of $\mathrm{CaCl}_{2}$ also enhanced the potency of plakortide-T. The synergy observed with $\mathrm{CaCl}_{2}$ was greater than what was observed for $\mathrm{ZnCl}_{2}$. The expected additive effect were calculated according to Spitzer et al. (143).

\subsection{EGTA failed to rescue Plakortide-T induced growth inhibition}

In an attempt to rescue plakortide-T induced growth inhibition by the expected increase in intracellular calcium, the PDR-deficient cells $(\Delta p d r 1 \Delta p d r 3)$ in media were pretreated with $2.5 \mu \mathrm{M}$ EGTA $\left(\sim \mathrm{IC}_{30}\right)$, a calcium ion chelator, before treating with plakortide-T in a dose response assay. The growth was determined by measuring the culture OD after treatment with plakortide-T and was compared against the sensitivity of the PDR-deficient cells treated with plakortide-T alone (Fig. 5.7). 
The sensitivity of the PDR-deficient cells pre-treated with EGTA before plakortide-T treatment was not significantly different from the PDR-deficient cells treated with plakortide-T alone. Therefore, no rescue of plakortide-T induced growth inhibition was observed from pre-treatment EGTA.

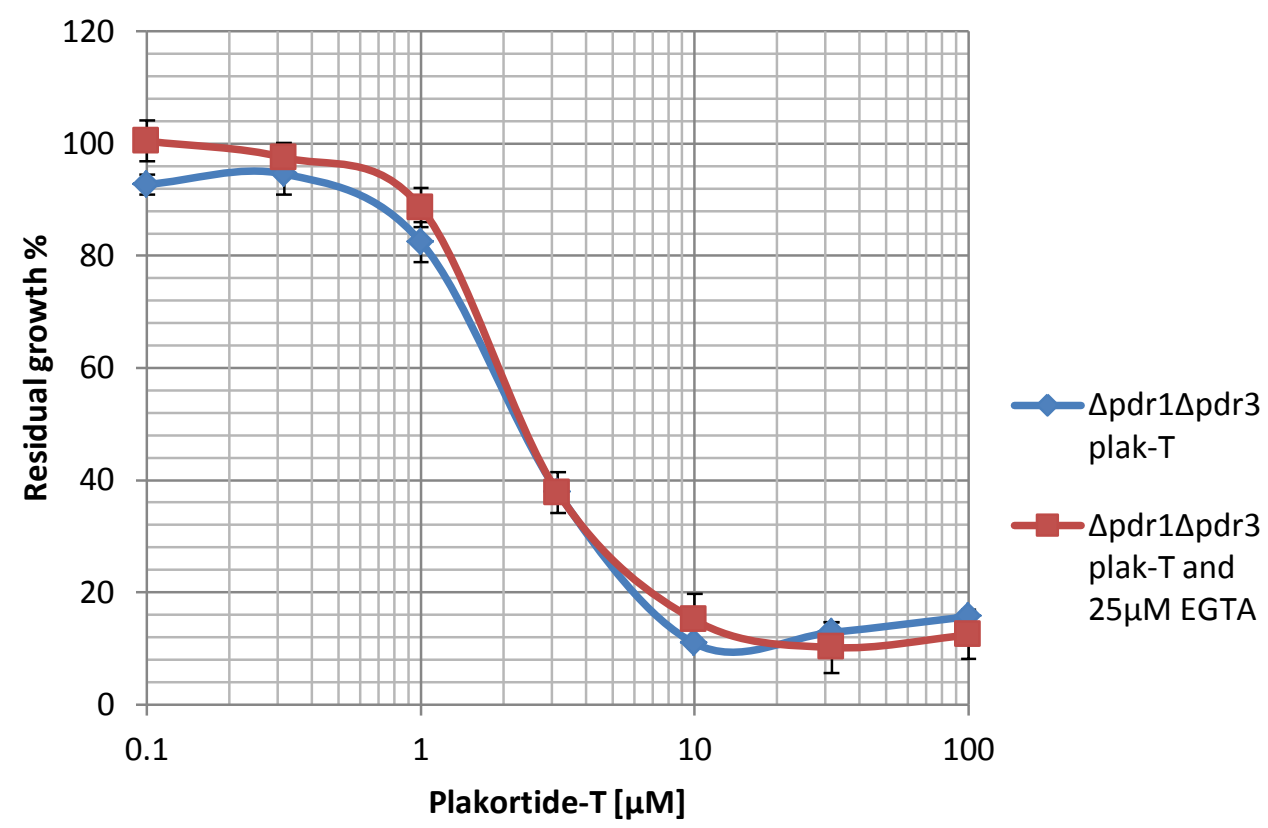

Figure 5.7 - EGTA rescue of plakortide-T induced growth inhibition. Dose response assays were performed on the PDR-deficient strain ( $\Delta p d r 1 \Delta p d r 3)$ against plakortide-T with EGTA and plakortide-T alone. The PDR-deficient strain had similar sensitivities to both plakortide with EGTA and plakortide-T alone.

5.10 The sensitivities of the $\mathrm{Ca}^{2+}$ and $\mathrm{Zn}^{2+}$ transporter mutants and calcineurin mutants to $\mathrm{CaCl}_{2}, \mathrm{ZnCl}_{2}$ and plakortide- $\mathrm{T}$

Addition of either $\mathrm{Ca}^{2+}$ or $\mathrm{Zn}^{2+}$ was shown to increase the potency of plakortide- $\mathrm{T}$ in a synergistic manner. The role of altered $\mathrm{Ca}^{2+}$ and $\mathrm{Zn}^{2+}$ homeostasis in mechanism of action of plakortide- $\mathrm{T}$ was assessed using the calcium transporter mutants: $\Delta v c x 1$, $\Delta p m c 1, \Delta p m r 1, \Delta c c h 1, \Delta m i d 1, \Delta y v c 1, \Delta e c m 7$; zinc transporter mutants: $\Delta z r t 1, \Delta z r t 3$, 
$\Delta z r t 2, \Delta z r c 1, \Delta \cot 1$ and calcineurin mutants: $\Delta c n b 1, \Delta c n a 1, \Delta c m p 2$ in the background of $\Delta p d r 1 \Delta p d r 3$ were used in dose response assays against $\mathrm{CaCl}_{2}, \mathrm{ZnCl}_{2}$ and PlakortideT. The growth was determined by measuring the culture OD and was compared against the sensitivity of the control strain $\Delta$ his3 (Fig. 5.8a-c).

In response to the $\mathrm{CaCl}_{2}$ treatment, the $\Delta p m r 1 \Delta p d r 1 \Delta p d r 3$ strain was significantly more sensitive to $\mathrm{CaCl}_{2}$ in comparison to the $\Delta h i s 3 \Delta p d r 1 \Delta p d r 3$ control strain. The other mutant strains assayed showed sensitivities that were not significantly different from $\Delta h i s 3 \Delta p d r 1 \Delta p d r 3$ control strain (Fig. 5.8a).

In response to the $\mathrm{ZnCl}_{2}$ treatment, all the mutant strains with the exception of $\Delta$ midl and $\Delta v c x l$ showed sensitivities that were not significantly different from $\Delta h i s 3 \Delta p d r 1 \Delta p d r 3$ control strain up to $3 \mu \mathrm{M} \mathrm{ZnCl}_{2}$ (Fig. 5.8b). At concentrations $10 \mathrm{mM}$ and higher $\mathrm{ZnCl}_{2}$ precipitated and its effect at higher concentrations could not be determined. The deletion strains $\Delta m i d 1 \Delta p d r 1 \Delta p d r 3$ and $\Delta v c x 1 \Delta p d r 1 \Delta p d r 3$ showed enhanced growth in the presence of semi-inhibitory concentrations of $\mathrm{ZnCl}_{2}$ (shown separately in Fig. 5.8c).

In response to plakortide-T, all the deletion strains showed sensitivities that were not significantly different from $\Delta h i s 3 \Delta p d r 1 \Delta p d r 3$ control strain (Fig. 5.8d). This shows that mutants defective in calcium and zinc homeostasis are not hypersensitive to plakortide-T treatment. 


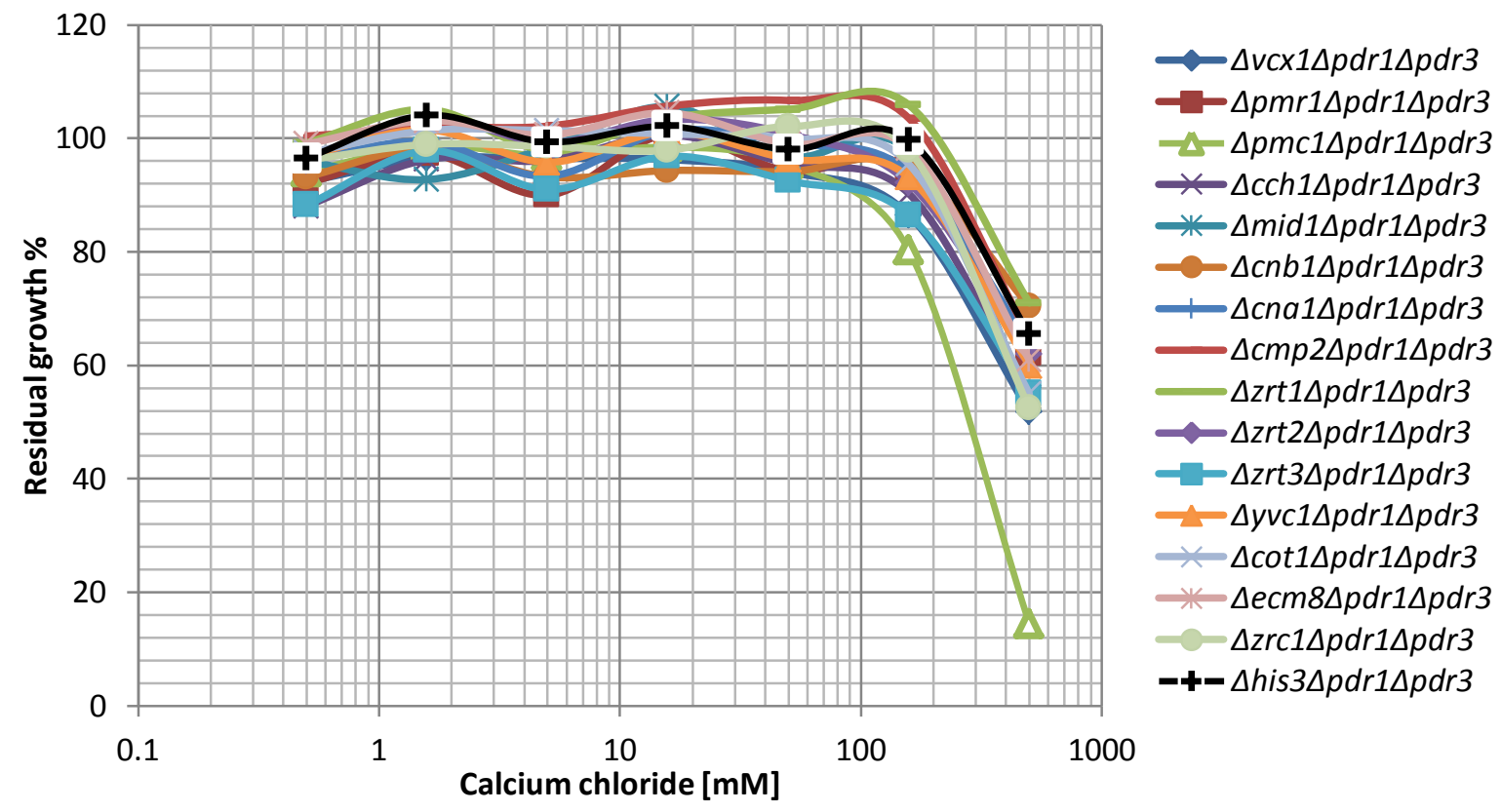

b

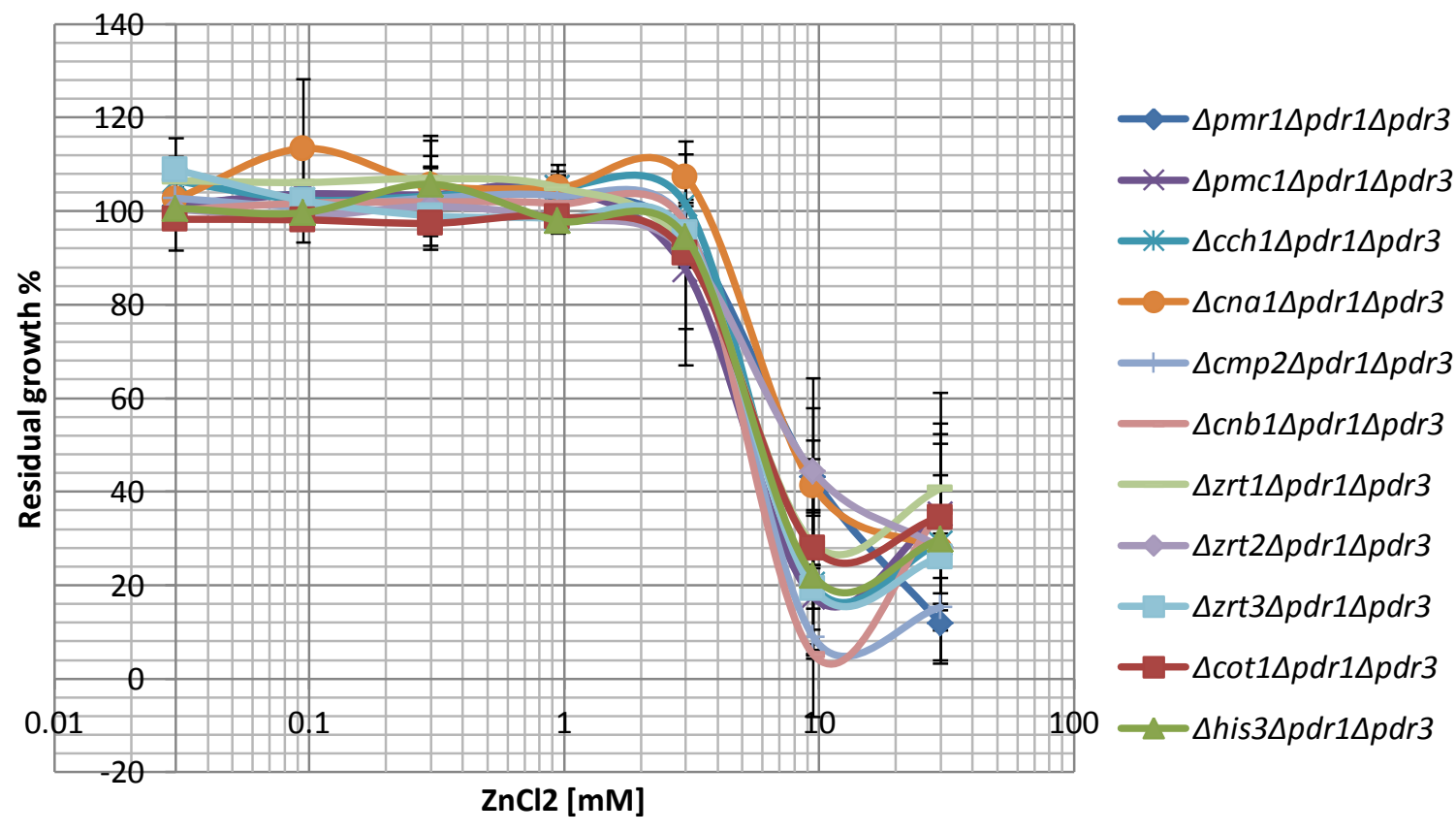



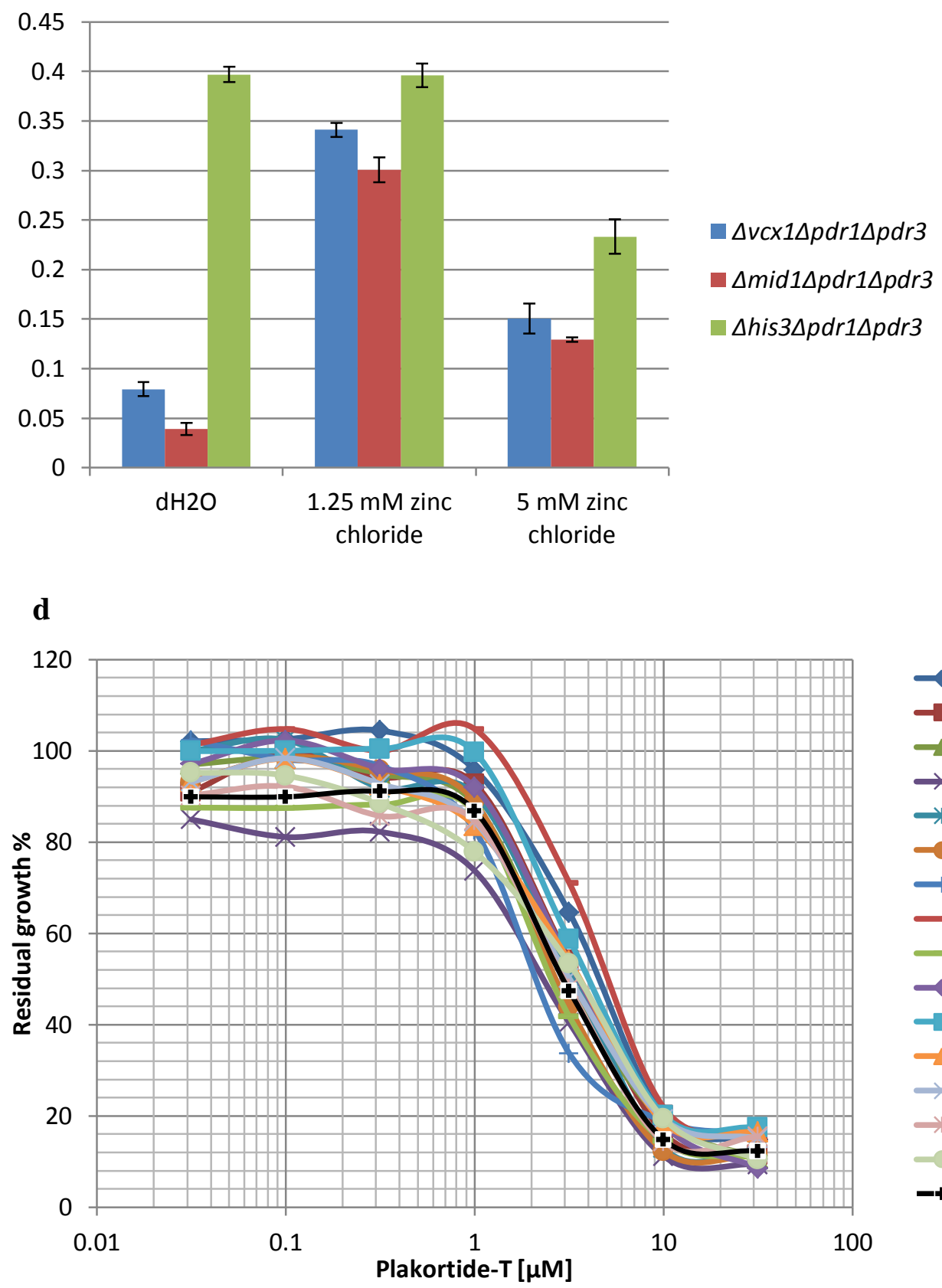

$-\Delta v c x 1 \Delta p d r 1 \Delta p d r 3$ $-\Delta p m r 1 \Delta p d r 1 \Delta p d r 3$ $-\Delta p m c 1 \Delta p d r 1 \Delta p d r 3$ $* \Delta c c h 1 \Delta p d r 1 \Delta p d r 3$ * $\Delta$ mid $1 \Delta p d r 1 \Delta p d r 3$ - $\Delta c n a 1 \Delta p d r 1 \Delta p d r 3$ $-\Delta c n b 1 \Delta p d r 1 \Delta p d r 3$ $\triangle c m p 2 \Delta p d r 1 \Delta p d r 3$ $\triangle z r t 1 \Delta p d r 1 \Delta p d r 3$ $\longrightarrow \Delta$ zrt2 $\Delta p d r 1 \Delta p d r 3$ $-\Delta z r t 3 \Delta p d r 1 \Delta p d r 3$ $-\Delta y v c 1 \Delta p d r 1 \Delta p d r 3$ $\times \Delta \cot 1 \Delta p d r 1 \Delta p d r 3$ * $\Delta$ ecm8 $\Delta p d r 1 \Delta p d r 3$ - $\Delta r c 1 \Delta p d r 1 \Delta p d r 3$ - $\Delta$ his3 $\Delta p d r 1 \Delta p d r 3$

Figure 5.8 - Sensitivities of calcium and transport mutants and calcineurin mutants to $\mathrm{CaCl}_{2}$, $\mathrm{ZnCl}_{2}$ and plakortide-T. A dose response assays was performed on PDR-deficient strains particularly, $\Delta v c x 1, \Delta p m c 1, \Delta p m r 1, \Delta c c h 1, \Delta$ mid1, $\Delta y v c 1, \Delta e c m 7, \Delta z r t 1, \Delta z r t 3, \Delta z r t 2, \Delta z r c 1$, $\Delta \cot 1, \Delta c n b 1, \Delta c n a 1, \Delta c m p 2$ and $\Delta$ his3 against $\mathrm{CaCl}_{2}, \mathrm{ZnCl}_{2}$ and plakortide- T. (a) The $\Delta p m r 1 \Delta p d r 1 \Delta p d r 3$ strain was the most sensitive to $\mathrm{CaCl}_{2}$ and the remaining deletion strains had sensitivities similar to $\Delta h i s 3 \Delta p d r 1 \Delta p d r 3$ control strain. (b) All the PDR-deficient deletion strains showed similar sensitivities to $\mathrm{ZnCl}_{2}$. (c) $\Delta \mathrm{vcx} 1$ and $\Delta$ mid1 showed enhanced growth phenotype to sub-lethal concentration of $\mathrm{ZnCl}_{2}$ in comparison to the his3 control. (d) All the PDR-deficient deletion strains showed similar sensitivities plakortide-T. 


\subsection{Deletion mutants hypersensitive to plakortide-T}

The sensitivities of the haploid PD-DMA ( $\Delta p d r 1 \Delta p d r 3 \Delta x x x)$ to plakortide-T were assessed as a pool by DNA barcode microarrays (Chapter 2 Materials and Methods). The competitive hybridisation ( $\mathrm{Cy} 5 / \mathrm{Cy} 3$ ratios) of barcodes denoting the different strains in plakortide-T treated and untreated conditions to microarray chip was used to measure the competitive fitness of each PDR-deficient strain within the pool.

The DNA barcode microarray experiment identified 55 phenotypic enhancements with plakortide-T (Table 5.3). There were no enrichments within the three main GO categories "biological process", "molecular function" or "cellular component". Therefore, Go slim categories on SGD were utilised which uses more general terms to define the biology of a gene product in terms of its biological process, molecular function and cellular component. The GO slim categories identified several biological processes affected by plakortide- $\mathrm{T}$ which included protein phosphorylation, membrane transport, lipid metabolic processes, cell cycle regulation, response to chemical stress and transcription from RNA polymerase II promoter. The GO slim categories also identified genes involved in several cellular components (Table 5.3), and enrichment was seen for mitochondrion localisation. 


\begin{tabular}{|c|c|c|c|}
\hline ORF & Gene & Component & Description \\
\hline YHR198C & AIM18 & Mito & Putative protein of unknown function \\
\hline YDR380W & ARO10 & Other & Phenylpyruvate decarboxylase \\
\hline YMR116C & ASC1 & Ribo & $\begin{array}{l}\text { G-protein beta subunit and guanine nucleotide } \\
\text { dissociation inhibitor }\end{array}$ \\
\hline YMR119W & ASI1 & $\begin{array}{l}\text { EmS, ER, } \\
\text { Nucl }\end{array}$ & Putative integral membrane E3 ubiquitin ligase \\
\hline YNL259C & ATX1 & Other & Cytosolic copper metallochaperone \\
\hline YGR224W & AZR1 & PM & $\begin{array}{l}\text { Plasma membrane transporter of the major } \\
\text { facilitator superfamily }\end{array}$ \\
\hline YER048C & CAJ1 & Nucl & $\begin{array}{l}\text { Nuclear type II J heat shock protein of the E. coli } \\
\text { dnaJ family }\end{array}$ \\
\hline YOR039W & CKB2 & Nucl & Beta' regulatory subunit of casein kinase 2 (CK2) \\
\hline YIL157C & COA1 & Nucl, Mito & $\begin{array}{l}\text { Mitochondrial membrane protein required for } \\
\text { assembly of complex IV }\end{array}$ \\
\hline YJR048W & CYC1 & Mito & Cytochrome c \\
\hline YDL174C & DLD1 & Mito & D-lactate dehydrogenase \\
\hline YLR214W & FRE1 & PM & Ferric reductase and cupric reductase \\
\hline YDL100C & GET3 & ER & Guanine nucleotide exchange factor for Gpa1p \\
\hline YNL281W & $\mathrm{HCH} 1$ & Nucl & Heat shock protein regulator that binds to Hsp90p \\
\hline YIR036C & IRC24 & Other & Putative benzil reductase \\
\hline YOR123C & LEO1 & Nucl & Component of the Paf1 complex \\
\hline YKL150W & MCR1 & Mito & Mitochondrial NADH-cytochrome b5 reductase \\
\hline YNL277W & MET2 & Other & L-homoserine-O-acetyltransferase \\
\hline YJR077C & MIR1 & Mito & Mitochondrial phosphate carrier \\
\hline YOR231W & MKK1 & CB, SoPG & $\begin{array}{l}\text { MAPKK involved in the protein kinase } C \text { signalling } \\
\text { pathway }\end{array}$ \\
\hline YOR188W & MSB1 & $\begin{array}{l}\text { CB, Mito, } \\
\text { SoPG }\end{array}$ & $\begin{array}{l}\text { Protein involved in regulating 1,3-beta-glucan } \\
\text { synthesis }\end{array}$ \\
\hline YLR338W & OPI9 & Unknown & $\begin{array}{l}\text { Dubious open reading frame unlikely to encode a } \\
\text { functional protein }\end{array}$ \\
\hline YDR466W & PKH3 & Unknown & $\begin{array}{l}\text { Protein kinase with similarity to mammalian PDK1 } \\
\text { and yeast Pkh1p/Phk2p }\end{array}$ \\
\hline YGL205W & POX1 & Prox & Fatty-acyl coenzyme A oxidase \\
\hline
\end{tabular}




\begin{tabular}{|c|c|c|c|}
\hline YHR076W & PTC7 & $\begin{array}{l}\text { EmS, Mito, } \\
\text { Nucl }\end{array}$ & Type $2 \mathrm{C}$ protein phosphatase (PP2C) \\
\hline YBR073W & RDH54 & Nucl & DNA-dependent ATPase \\
\hline YER047C & SAP1 & Other & Putative ATPase of the AAA family \\
\hline YDR409W & SIZ1 & $\begin{array}{l}\text { Ccort, } \\
\text { Csome, Nucl }\end{array}$ & $\begin{array}{l}\text { SUMO/Smt3 ligase that promotes the attachment } \\
\text { of sumo }\end{array}$ \\
\hline YBR182C & SMP1 & Nucl & $\begin{array}{l}\text { Putative transcription factor involved in osmotic } \\
\text { stress response }\end{array}$ \\
\hline YAL030W & SNC1 & $\begin{array}{l}\mathrm{CB}, \mathrm{EmS}, \mathrm{PM} \\
\text { SoPG }\end{array}$ & Vesicle membrane receptor protein ( $V$-SNARE) \\
\hline YHR014W & SPO13 & Csome, Nucl & Meiosis-specific protein \\
\hline YIL073C & SPO22 & Csome, Nucl & $\begin{array}{l}\text { Meiosis-specific protein essential for chromosome } \\
\text { synapsis }\end{array}$ \\
\hline YDR463W & STP1 & Nucl & Transcription factor \\
\hline YDR297W & SUR2 & EmS, ER, & Sphinganine C4-hydroxylase \\
\hline YML072C & TCB3 & $\begin{array}{l}\text { Ccort, } \quad C B \text {, } \\
\text { ER, Mito }\end{array}$ & $\begin{array}{l}\text { Lipid-binding ER protein, enriched at ER-plasma } \\
\text { membrane contact sites }\end{array}$ \\
\hline YBR118W & TEF2 & Ribo & Translational elongation factor EF-1 alpha \\
\hline YJR019C & TES1 & Mito, Prox & Peroxisomal acyl-CoA thioesterase \\
\hline YBL054W & TOD6 & Csome, Nucl & $\begin{array}{l}\text { PAC motif binding protein involved in rRNA and } \\
\text { ribosome biogenesis }\end{array}$ \\
\hline YDL210W & UGA4 & Vac & GABA (gamma-aminobutyrate) permease \\
\hline YNL054W & VAC7 & Vac & $\begin{array}{l}\text { Integral vacuolar membrane protein involved in } \\
\text { vacuole organization }\end{array}$ \\
\hline YDR247W & VHS1 & Other & Cytoplasmic serine/threonine protein kinase \\
\hline YOR069W & VPS5 & EmS, & Nexin-1 homolog \\
\hline YAL042C-A & YAL042C-A & Other & Dubious open reading frame \\
\hline YEL010W & YEL010W & Unknown & Dubious open reading frame \\
\hline YHR022C & YHR022C & Unknown & Putative protein of unknown function \\
\hline YIR042C & YIR042C & Unknown & Putative protein of unknown function \\
\hline YMR262W & YMR262W & Unknown & Protein of unknown function \\
\hline $\begin{array}{l}\text { YMR316C- } \\
\text { A }\end{array}$ & $\begin{array}{l}\text { YMR316C- } \\
\text { A }\end{array}$ & Unknown & Protein of unknown function \\
\hline YNL057W & YNL057W & Unknown & Dubious open reading frame \\
\hline
\end{tabular}




\begin{tabular}{|l|l|l|l|}
\hline YNL120C & YNL120C & Unknown & Dubious open reading frame \\
\hline YNL285W & YNL285W & Unknown & Dubious open reading frame \\
\hline YOL036W & YOL036W & Unknown & Protein of unknown function \\
\hline YOL092W & YOL092W & Vac, Ribo & Putative protein of unknown function \\
\hline YOR019W & YOR019W & Ribo & Protein of unknown function \\
\hline YOR376W & YOR376W & Other & Dubious open reading frame. \\
\hline
\end{tabular}

Table 5.3 - List of genes deletion mutants hypersensitive to $160 \mathrm{nM}$ plakortide-T in a barcode microarray experiment. 55 phenotypic enhancements were identified from a DNA barcode microarray utilizing the haploid PDR-deficient mutants. Genes were annotated according to SGD (62). Highlighted in blue are genes encoding for components of the mitochondrion. Mitochondrion (Mito), Chromosome (Csome), Ribosome (Ribo), Endomembrane System (EmS), Endoplasmic Reticulum (ER), Nucleus (Nucl), Plasma Membrane (PM), Cellular Bud (CB), Site of Polarized Growth (SoPG), Peroxisome (Prox), Cell cortex (Ccort), Vacuole (Vac).

\section{$\underline{5.12}$ Plakortide-T does not induce the oxidative stress response regulator YAP1}

Mitochondria are one of the main contributors and targets of ROS. The loss of function mutations of several mitochondrial protein encoding genes determined as hypersensitive to plakortide-T and mitochondrial damage as an indicator of ROS generation makes a strong case for reactive oxygen species generation by plakortide- $\mathrm{T}$.

The determine ROS generation, PDR-deficient strain, $\triangle p d r 1 \Delta p d r 3$ YAP1-GFP was treated with plakortide-T and known ROS generating compounds, menadione and $\mathrm{H}_{2} \mathrm{O}_{2}$. The activation of Yap1p was detected by nuclear localisation of GFP using confocal microscopy (Fig. 5.9). Yap1p-GFP had a cytoplasmic location under untreated conditions and immediate nuclear localisation of Yap1p-GFP was observed for the PDR-deficient strain following treatment with menadione and $\mathrm{H}_{2} \mathrm{O}_{2}$. No nuclear 
localisation of Yap1p-GFP was observed with following treatment with plakortide-T. This demonstrates that plakortide-T does not induce the oxidative stress response.

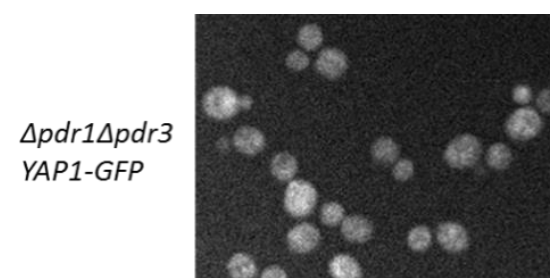

$\mathrm{dH}_{2} \mathrm{O}$

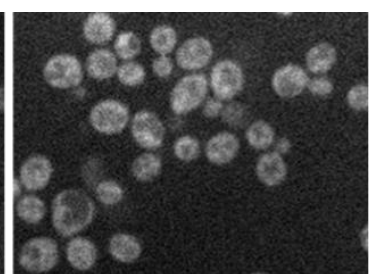

$100 \mu \mathrm{M}$ Plackortide T

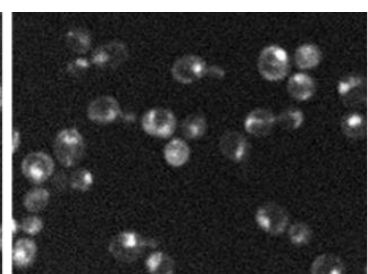

$100 \mu \mathrm{M}$ Mennadione

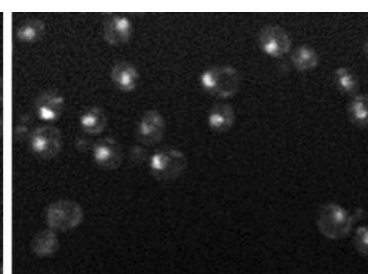

$1 \mathrm{mM} \mathrm{H}_{2} \mathrm{O}_{2}$

Figure 5.9 - Plakortide-T does not induce the oxidative stress response regulator Yap1p. The PDR-deficient strain, $\triangle p d r 1 \triangle p d r 3 Y A P 1-G F P$ was treated with the highest concentration of plakortide-T and ROS generating compounds menadione and $\mathrm{H}_{2} \mathrm{O}_{2}$ at MIC. The images were cropped for presentation using Image J (rsbweb.nih.gov/ij/). Nuclear localisation of Yap1p was observed for the PDR-deficient strain, $\triangle p d r 1 \triangle p d r 3 Y A P 1-G F P$, in response to treatment with menadione and $\mathrm{H}_{2} \mathrm{O}_{2}$ but not with plakortide-T.

\subsection{Plakortide-T does not generate ROS detectable by DTNB}

Oxidation of free thiols on proteins was investigated as another approach to detect ROS generation. The PDR-deficient strain, $\Delta p d r 1 \Delta p d r 3$, was treated with menadione, plakortide-T and DMSO and the proteins were extracted with TCA treatment and centrifugation. The free thiols on proteins were reacted with DTNB (Ellman's reagent) and the resulting colour change was measured by optical density of the sample at 412 $\mathrm{nM}$ (Fig. 5.10)

The $\mathrm{OD}$ of the menadione treated sample $\left(\mathrm{OD}_{412} 0.03\right)$ was significantly reduced from what was observed for DMSO treated sample $\left(\mathrm{OD}_{412}\right.$ 0.054). As $\mathrm{OD}$ measures the 
number of thiols present in the sample, the reduced OD for menadione suggests an increased oxidation of thiols due to reaction with ROS generation. Therefore, menadione is an effective control for ROS generation assays. The OD of the plakortide$\mathrm{T}$ treated sample was not significantly different from the DMSO treated control $\left(\mathrm{OD}_{412}\right.$ 0.054). This shows no additional oxidation of free thiols with plakortide-T treatment in comparison to the DMSO treated control.

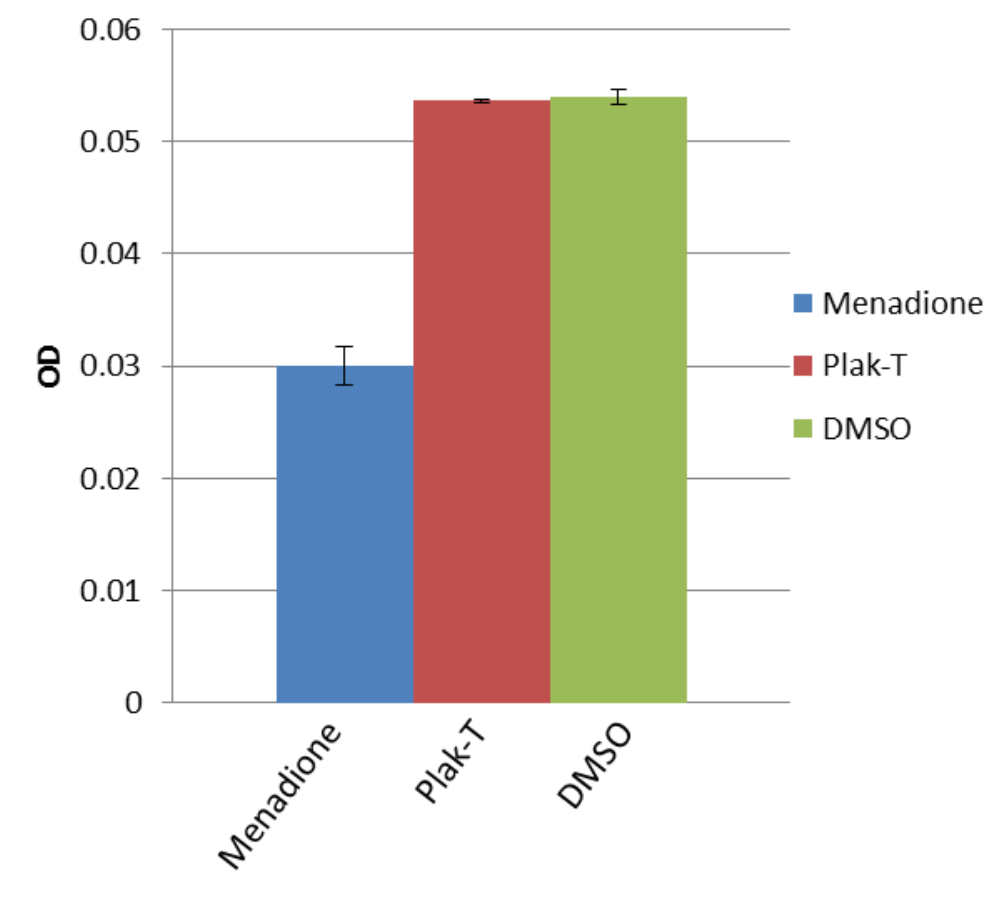

Figure 5.10 - Plakortide-T does not generate ROS detectable by the DTNB assay. The PDRdeficient strain, $\Delta p d r 1 \Delta p d r 3$ was treated with plakortide-T $(100 \mu \mathrm{M})$, menadione $(100 \mu \mathrm{M})$ and carrier solvent DMSO and the proteins were extracted from each of the samples and were reacted with DNTB. OD in each sample was determined which is relative to the amount of free thiols. The amount of free thiols in plakortide-T treated sample was similar to the DMSO treated control. The significant reduction in the amount of free thiols was observed with menadione in comparison to the DMSO treated control. 
5.14 CRZ1-GFP as a reporter strain for increased intracellular $\mathrm{Ca}^{2+}$ levels.

The subcellular localisation of the calcium responsive transcription factors Crzlp, Hog1p and Msn2p were assessed in response to increased cytosolic calcium. The GFP reporter strains, CRZ1-GFP, HOG1-GFP and MSN2-GFP were treated with $\mathrm{CaCl}_{2}$ and the nuclear localisation of Crz1p-GFP, Hog1p-GFP and Msn2p-GFP fusion proteins in response to increased cytosolic $\mathrm{Ca}^{2+}$ were detected by confocal microscopy (Fig. 5.11).

The GFP fusion proteins Crz1p-GFP and Hog1p-GFP were cytoplasmic under untreated conditions, while MSN2-GFP strain showed some cells with nuclear localised Msn2pGFP under untreated conditions. The nuclear localisation of Hog1p-GFP was observed only at $400 \mathrm{mM} \mathrm{CaCl} 2$ and only in some of the cells and it was faint (Fig.5.11b). At 150-300 mM concentrations of $\mathrm{CaCl}_{2}$, no nuclear localisation of Hog1-GFP was observed. The HOG1-GFP strain therefore, was not an effective reporter for increased cytosolic $\mathrm{Ca}^{2+}$. The nuclear localisation of $\mathrm{Msn} 2 \mathrm{p}$ under $\mathrm{CaCl}_{2}$ treated conditions although were easily distinguishable by bright nuclear localised GFP, a large proportion of cells still showing cytoplasmic location of Msn2p-GFP even at highest concentration of $\mathrm{CaCl}_{2}$ used (Fig.5.11c). The proportion of cells with nuclear location of Msn2p-GFP increased in a concentration dependent manner to $\mathrm{CaCl}_{2}$. The nuclear localisation of Msn2p-GFP in the absence of $\mathrm{CaCl}_{2}$ which suggests it may be up-regulated in response to several other signals as well as an increase in cytoplasmic calcium. The MSN2-GFP strain therefore, was not an effective reporter for increased cytosolic $\mathrm{Ca}^{2+}$.

In contrast, nuclear localisation of $C r z 1 p-G F P$ was observed from $150 \mathrm{mM}-400 \mathrm{mM}$ concentrations of $\mathrm{CaCl}_{2}$ and was easily distinguishable from untreated control cells which showed cytoplasmic location for Crz1p-GFP (Fig.5.11a). At lower $150 \mathrm{mM}$ 
$\mathrm{CaCl}_{2}$ concentrations, a small proportion of $C R Z 1-G F P$ cells still showed cytoplasmic location of Crz1p-GFP. In contrast, all the cells showed nuclear localisation of Crz1pGFP at higher $300 \mathrm{mM}$ and $400 \mathrm{mM}$ concentrations of $\mathrm{CaCl}_{2}$. Therefore, CRZ1-GFP strain was an effective reporter for increased intracellular calcium at $300 \mathrm{mM} \mathrm{CaCl} 2$ and was employed in subsequent experiments as the concentration of to induce the increased cytosolic $\mathrm{Ca}^{2+}$ response.

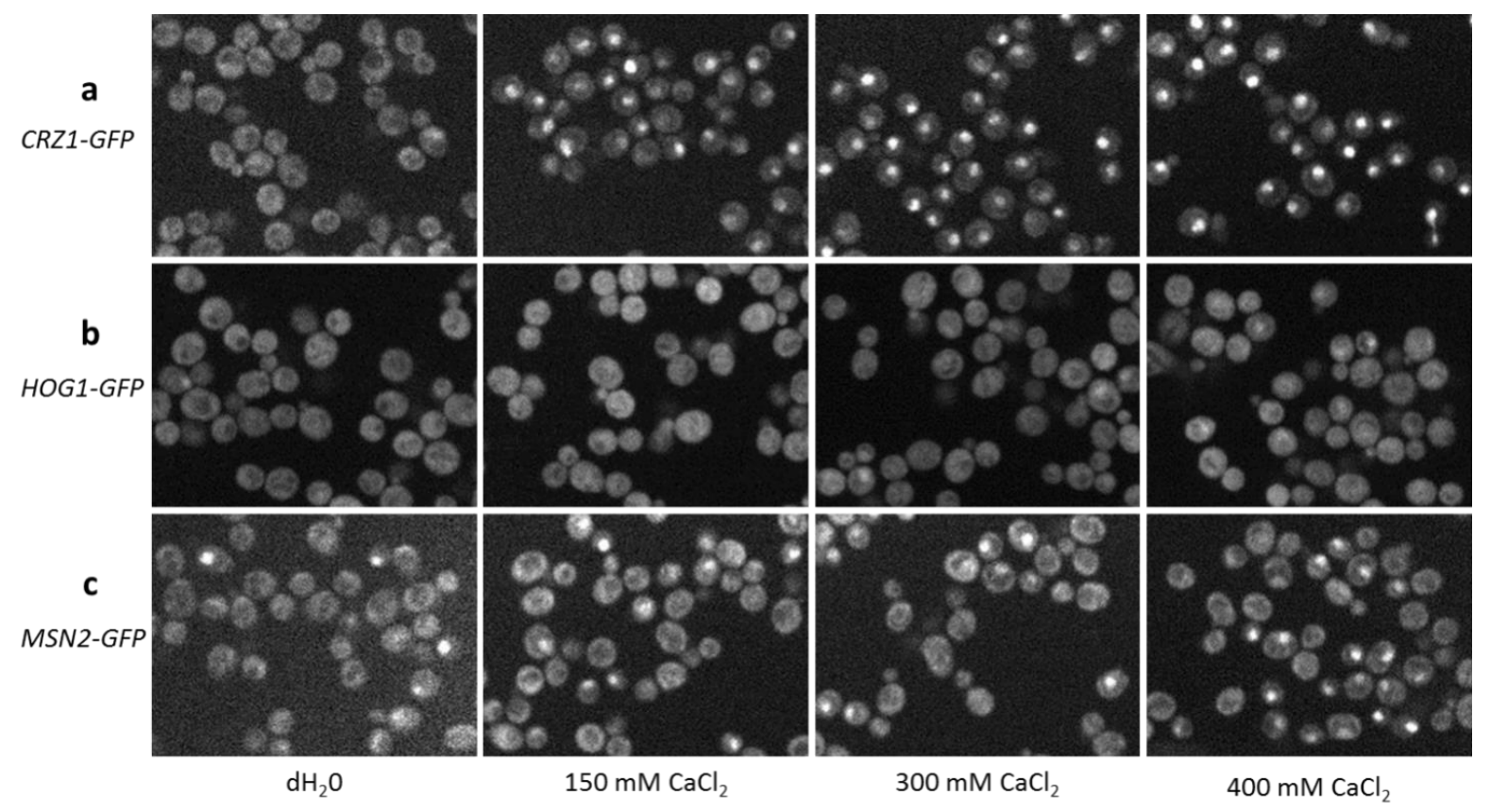

Figure 5.11 - Crz1p-GFP as reporter for increase in intracellular calcium. GFP strains CRZ1GFP, HOG1-GFP and MSN2-GFP were treated with $150 \mathrm{mM}, 300 \mathrm{mM}$ and $400 \mathrm{mM} \mathrm{CaCl}_{2}$ and were incubated for $4 \mathrm{~h}$ at $30^{\circ} \mathrm{C}$. The cells were visualised using the OPERA confocal microscope. The images were brightness/contrast adjusted and cropped for presentation using Image J (rsbweb.nih.gov/ij/). (a) Nuclear localisation of Crz1p-GFP was observed at all of the $\mathrm{CaCl}_{2}$ concentrations and no nuclear localisation of GFP was observed for the untreated control. (b) A few cells showed a nuclear localisation of HOG1-GFP at the highest concentration of $400 \mathrm{mM} \mathrm{CaCl}_{2}$ but not at the other concentration of $\mathrm{CaCl}_{2}$. (c) A few cells showed nuclear localisation of Msn2p-GFP across under $\mathrm{CaCl}_{2}$ treated conditions and also in the untreated condition. 
5.15 A reporter for increased intracellular $\mathrm{Zn}^{2+}$ levels.

To distinguish increased cytosolic $\mathrm{Ca}^{2+}$ from increased cytosolic $\mathrm{Zn}^{2+}$, the zinc responsive transcription factor Zap1p was utilised. The GFP fusion strain ZAP1-GFP was treated with $\mathrm{ZnCl}_{2}$ and $\mathrm{CaCl}_{2}$ and the subcellular localisation of $\mathrm{Zap} 1 \mathrm{p}-\mathrm{GFP}$ was detected by confocal microscopy (Fig. 5.12). The CRZ1-GFP strain was included as a negative control.

The GFP fusion protein, Zap1p-GFP showed nuclear localisation in untreated cells and no change GFP localisation was observed following treatment with $\mathrm{ZnCl}_{2}$ or $\mathrm{CaCl}_{2}$ (Fig. 5.12b). Therefore, this strain was not an effective control for increased cytosolic $\mathrm{Zn}^{2+}$.

The Crzlp-GFP responded as expected to $300 \mathrm{mM} \mathrm{CaCl}_{2}$ with nuclear relocation of cytoplasmic Crz1p-GFP. However Crz1p-GFP, showed nuclear localisation in several cells following treatment with $\mathrm{ZnCl}_{2}$ (Fig. 5.12a). The number of cells showing nuclear localisation of Crz1p-GFP increased with the concentration of $\mathrm{ZnCl}_{2}$ used, with $\sim 60 \%$ of the cells showing nuclear localisation of Crz1p-GFP at $40 \mathrm{mM} \mathrm{ZnCl}_{2}$. To investigate the nuclear localisation of Crzlp-GFP in response to general stress mechanisms, the CRZ1-GFP strain was treated with latrunculin-A $\left(\sim \mathrm{IC}_{30}\right)$ (Fig. 5.12a). No nuclear localisation of Crz1p-GFP was observed with latrunculin-A. This shows that Crz1pGFP localisation to the nucleus following $\mathrm{CaCl}_{2}$ and $\mathrm{ZnCl}_{2}$ treatment was not due to general stress response but rather specific to increased cytosolic $\mathrm{Ca}^{2+}$ and increased cytosolic $\mathrm{Zn}^{2+}$. 
To identify a reporter which responds only to $\mathrm{Zn}^{2+}$ and not $\mathrm{Ca}^{2+}$, the $\mathrm{Zn}^{2+}$ transporter Zrc1p which pumps cytosolic $\mathrm{Zn}^{2+}$ into the vacuole was investigated (Fig. 5.12c-d). The ZRC1-GFP strain was treated with $\mathrm{CaCl}_{2}$ and $\mathrm{ZnCl}_{2}$ and the expression of ZRC1GFP was detected by GFP intensity using OPERA confocal microscopy and Acapella. The GFP expression in $\mathrm{ZnCl}_{2}$ treated cells did not differ significantly from GFP expression in untreated cells. Therefore, no significant induction of Zrc1p-GFP was observed in response to increased cytosolic $\mathrm{Zn}^{2+}$. 

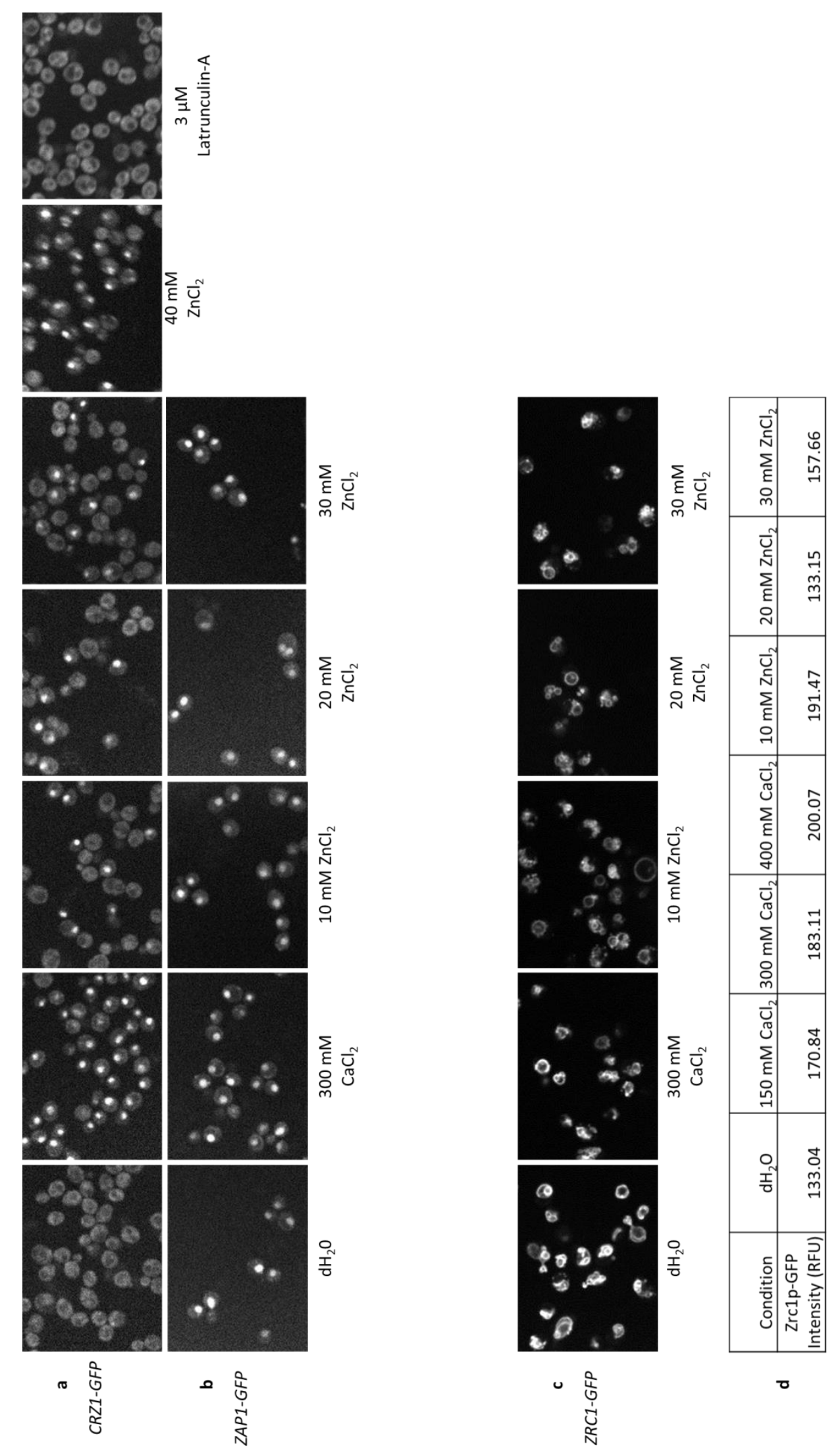
Figure 5.12 - Reporter for increase in intracellular $\mathrm{Zn}^{2+}$. GFP reporter strains CRZ1-GFP, ZAP1GFP and ZRC1-GFP were treated with $300 \mathrm{mM} \mathrm{CaCl}_{2}, 10 \mathrm{mM} \mathrm{ZnCl}_{2}, 20 \mathrm{mM} \mathrm{ZnCl} 2,30 \mathrm{mM} \mathrm{ZnCl}$. In addition CRZ1-GFP was also treated with $40 \mathrm{mM} \mathrm{ZnCl} 2$ and $3 \mu \mathrm{M}$ latrunculin-A and cells were incubated for $4 \mathrm{~h}$ at $30^{\circ} \mathrm{C}$. The cells were visualised using the confocal microscope. The images were brightness/contrast adjusted and cropped using Image J (rsbweb.nih.gov/ij/) to construct the above figure. (a) Crz1p-GFP showed nuclear localisation following treatment with $300 \mathrm{mM} \mathrm{CaCl}_{2}$ and a small proportion of the cells also showed nuclear localization of Crz1p-GFP in response to $\mathrm{ZnCl}_{2}$ treatment. (b) The Zap1p-GFP showed nuclear localisation in cells across all the conditions including untreated. (c-d) The GFP intensity for each of the ZRC1GFP cells in each well was measured using Acapella and averaged GFP intensities for duplicate experiments are given in the table above. No significant change in Zrc1p-GFP was observed in $\mathrm{CaCl}_{2}$ or $\mathrm{ZnCl}_{2}$ treatment.

5.16 Plakortide- $\mathrm{T}$ induces either increased intracellular $\mathrm{Ca}^{2+}$ or $\mathrm{Zn}^{2+}$ or both

The CRZ1-GFP reporter strain was introduced to the background of $\Delta p d r 1 \Delta p d r 3$ and changes in the subcellular localisation of Crz1p-GFP following treatment with plakortide- $\mathrm{T}, \mathrm{CaCl}_{2}$ or $\mathrm{ZnCl}_{2}$ was assayed using confocal microscopy (Fig. 5.13). The GFP fusion protein Crzlp-GFP was cytoplasmic under untreated conditions and following treatment with $\mathrm{CaCl}_{2}$ all cells showed nuclear localisation of Crz1p-GFP. This was consistent which what was observed for the CRZ1-GFP reporter strain the wild-type background (Fig. 5.11).

In response to $\mathrm{ZnCl}_{2}, \sim 65 \%$ of the cells showed nuclear localisation of Crz1p-GFP while the remaining cells showed cytoplasmic location Crzlp-GFP. This was consistent which what was observed for the CRZ1-GFP reporter strain the wild-type background (Fig. 5.12). 
In response to plakortide-T, cytoplasmic Crz1p-GFP localised to the nucleus at all the concentrations of plakortide-T tested. A small number of cell showed cytoplasmic localisation of Crz1p-GFP at low concentrations, however, all cells showed nuclear localisation of Crz1p-GFP at $15 \mu \mathrm{M}$ plakortide-T. The nuclear localisation of Crz1pGFP in response to plakortide- $T$ treatment confirms that plakortide- $T$ causes an increase in cytosolic calcium or $\mathrm{ZnCl}_{2}$.

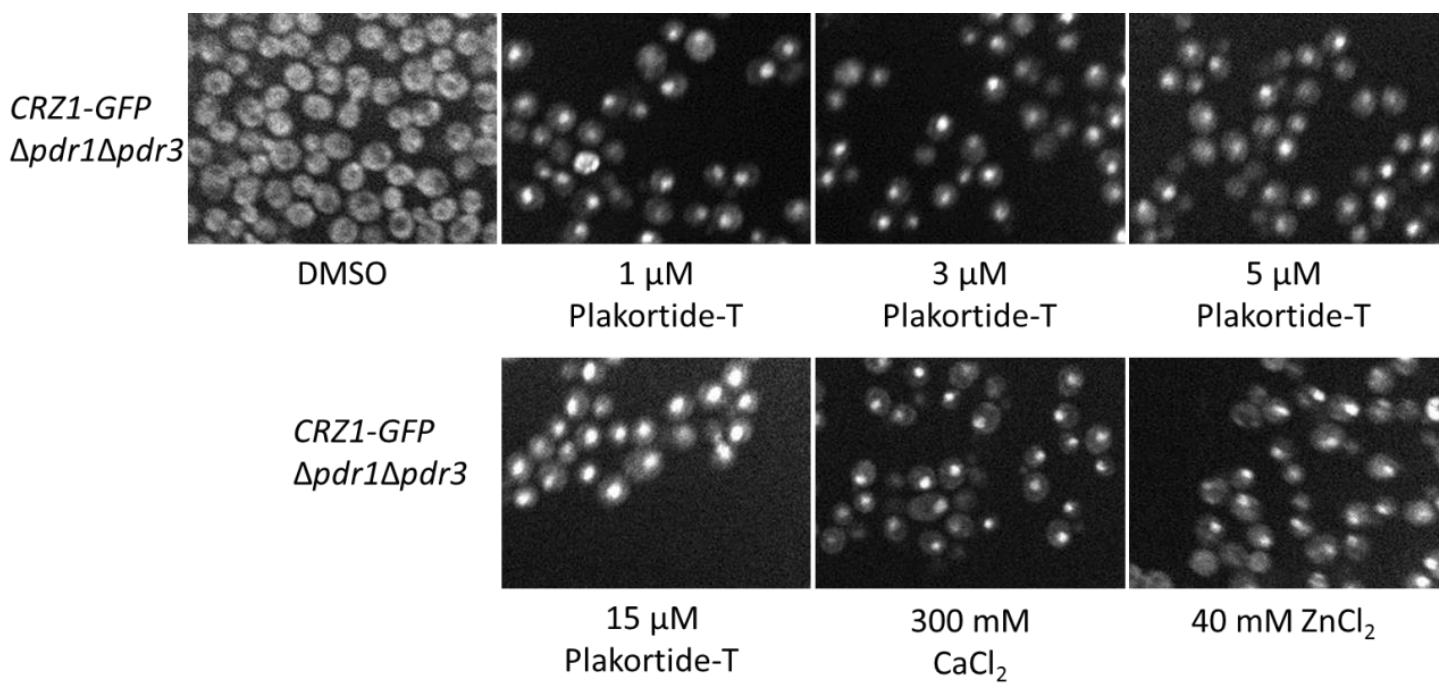

Figure 5.13 - Plakortide-T treatment causes an increase in cytosolic $\left[\mathrm{Ca}^{2+}\right]$. CRZ1-GFP $\Delta p d r 1 \Delta p d r 3$ strain was treated with $1 \mu \mathrm{M}, 3 \mu \mathrm{M}, 5 \mu \mathrm{M}$ and $15 \mu \mathrm{M}$ plakortide-T; $300 \mathrm{mM} \mathrm{CaCl}_{2}$ and $40 \mathrm{mM} \mathrm{ZnCl}_{2}$. The cells were visualised using confocal microscopy and the images were brightness/contrast adjusted and cropped for presentation using Image J (rsbweb.nih.gov/ij/). Nuclear localisation of Crz1p-GFP was observed at all of the plakortide-T concentrations tested. Some cells showed nuclear localisation of Crz1p-GFP with $40 \mathrm{mM} \mathrm{ZnCl}_{2}$ and all cells showed nuclear localisation of Crz1p-GFP with $\mathrm{CaCl}_{2}$. 
5.17 Location of Crz1p-GFP in $\mathrm{Ca}^{2+}$ and $\mathrm{Zn}^{2+}$ homeostasis mutants in response to plakortide- $\mathrm{T} \mathrm{CaCl}_{2}$ and $\mathrm{ZnCl}_{2}$ treatment

The calcium transporter mutants; $\Delta v c x 1, \Delta p m c 1, \Delta p m r 1, \Delta c c h 1, \Delta m i d 1, \Delta y v c 1, \Delta e c m 7$ and zinc transporter mutants; $\Delta z r t 1, \Delta z r t 3, \Delta z r t 2, \Delta z r c 1$ and $\Delta \cot 1$ and calcineurin mutants $\Delta c n b 1, \Delta c n a 1, \Delta c m p 2$ in the background of $\triangle p d r 1 \Delta p d r 3$ and $C R Z 1-G F P$ were utilised against plakortide- $T$ to further assess the effects of calcium and zinc homeostasis disruption (Fig 5.14).

The Crz1p-GFP in the $\Delta p m r 1 \Delta p d r 1 \Delta p d r 3 C R Z 1-G F P$ mutant had a cytoplasmic location in untreated conditions. The remainder of the mutants had an exclusively cytoplasmic location for Crz1p-GFP under untreated conditions and were similar to the $\Delta h i s 3 \Delta p d r 1 \Delta p d r 3$ CRZ1-GFP control strain. Following plakortide-T treatment all the mutant strains showed nuclear localisation of Crz1p-GFP with the exception of the calcineurin mutant strain, $\triangle c n b 1 \Delta p d r 1 \Delta p d r 3 C R Z 1-G F P$ and were similar to the control strain. The $\Delta c n b 1 \Delta p d r 1 \Delta p d r 3 C R Z 1-G F P$ mutant strain in contrast showed no nuclear localisation Crz1p-GFP in response to plakortide-T treatment and the reporter remained cytoplasmic. The nuclear localisation of Crz1p-GFP in the $\Delta p m r 1 \Delta p d r 1 \Delta p d r 3 C R Z 1$ GFP strain was not altered by plakortide-T treatment.

The observation with plakortide- $\mathrm{T}$ was consistent with $\mathrm{CaCl}_{2}$ or $\mathrm{ZnCl}_{2}$ where all strains with the exception of $\Delta c n b 1 \Delta p d r 1 \Delta p d r 3 C R Z 1-G F P$ showed nuclear location of Crz1pGFP. The Crz1p-GFP in the $\triangle c n b 1 \Delta p d r 1 \Delta p d r 3 C R Z 1-G F P$ mutant strain remained cytoplasmic in response to $\mathrm{CaCl}_{2}$ or $\mathrm{ZnCl}_{2}$ treatment. The nuclear localisation of 
$\Delta p m r 1 \Delta p d r 1 \Delta p d r 3 C R Z 1-G F P$ mutant strain was unaffected by either $\mathrm{CaCl}_{2}$ or $\mathrm{ZnCl}_{2}$ treatment. As previously observed with $\mathrm{ZnCl}_{2}$ treatment, only a few cells showed nuclear localisation of Crzlp-GFP and others remained in the cytoplasm. Interestingly slightly increased cell size was observed for cells treated with $\mathrm{ZnCl}_{2}$ which were not observed with $\mathrm{CaCl}_{2}$ or plakortide-T (data not shown). The absence of Crzlp-GFP nuclear localisation in the $\Delta c n b 1$ mutant in response to Plakortide-T, $\mathrm{CaCl}_{2}$ and $\mathrm{ZnCl}_{2}$ treatment shows that nuclear localisation of Crzlp-GFP is mediated through calcineurin since the deletion of the regulatory subunit Cnb1p disables this response. 


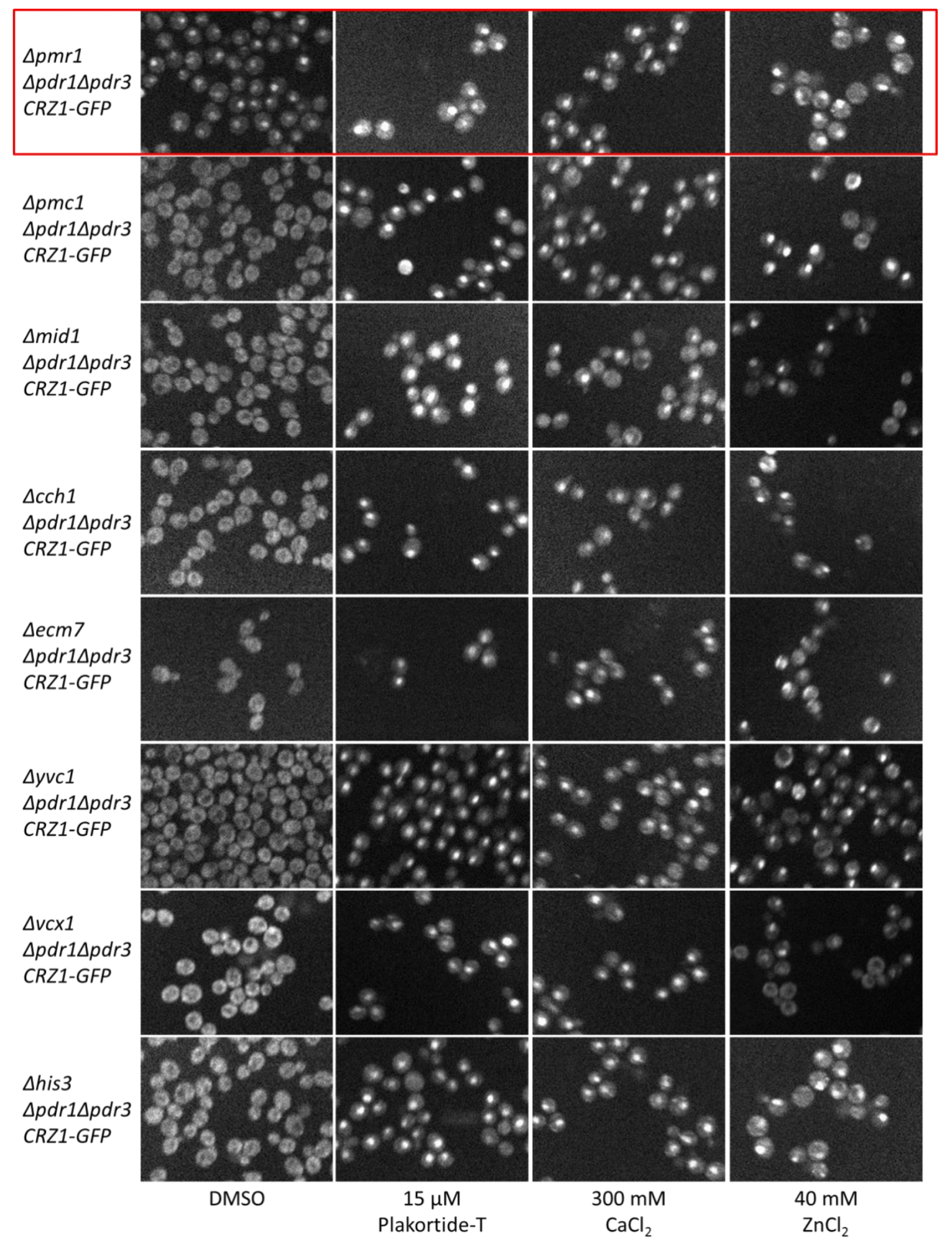




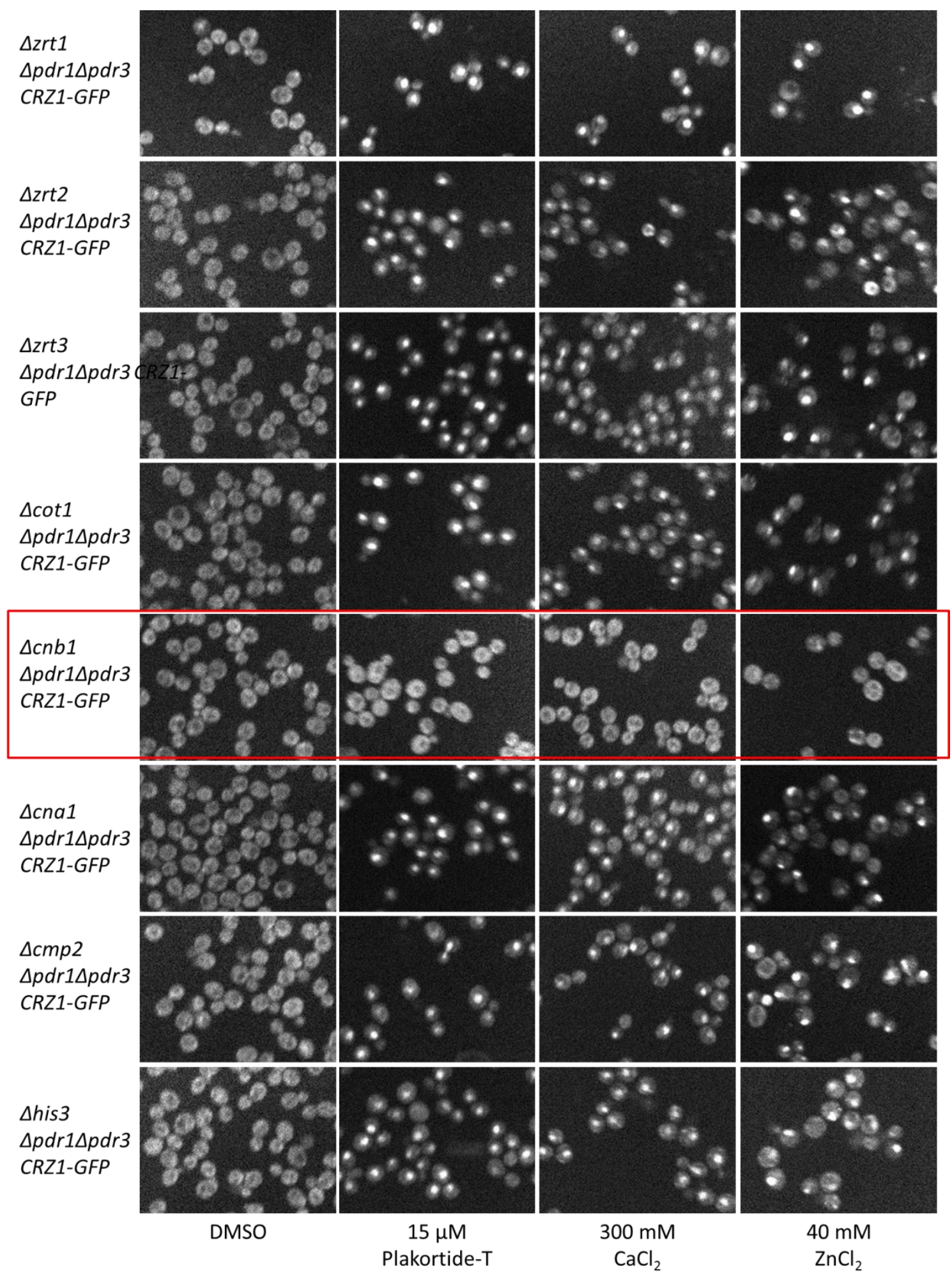

Figure 5.14 - Location of Crz1p-GFP in calcium and zinc homeostasis mutants in response to treatment with plakortide- $\mathrm{T}, \mathrm{CaCl}_{2}$ and $\mathrm{ZnCl}_{2}$. The calcium and zinc homeostasis mutants, $\Delta v c x 1, \Delta p m c 1, \Delta p m r 1, \Delta c c h 1, \Delta m i d 1, \Delta y v c 1, \Delta e c m 7, \Delta z r t 1, \Delta z r t 3, \Delta z r t 2, \Delta z r c 1, \Delta \cot 1, \Delta c n b 1$, $\triangle c n a 1, \Delta c m p 2$ and the control strain $\Delta h i s 3$ in the background of $\triangle p d r 1 \Delta p d r 3$ and CRZ1-GFP were treated with $15 \mu \mathrm{M}$ plakortide- $\mathrm{T}, 300 \mathrm{mM} \mathrm{CaCl}_{2}$ and $40 \mathrm{mM} \mathrm{ZnCl}_{2}$ and were incubated at 
$30^{\circ} \mathrm{C}$ for $4 \mathrm{~h}$. The cells were visualised using the OPERA confocal microscope. The images were brightness/contrast adjusted and cropped for presentation using Image J (rsbweb.nih.gov/ij/). The Crz1p-GFP fusion protein was exclusively cytoplasmic under DMSO treated in all the mutants with the exception of pmr1 $p p d r 1 \Delta p d r 3$ CRZ1-GFP mutant and was similar to the $\Delta$ his3 control. Nuclear localisation of Crz1p-GFP was observed for $15 \mu \mathrm{M}$ plakortide-T and 300 $\mathrm{mM} \mathrm{CaCl}_{2}$ and $40 \mathrm{mM} \mathrm{ZnCl}_{2}$ treated cells in most of the mutant strains. The only exception was the cnb1 $\Delta p d r 1 \Delta p d r 3$ CRZ1-GFP strain which showed no nuclear localisation of Crz1p-GFP under any of the conditions. The nuclear localisation of Crz1p-GFP was observed for nearly all cells in plakortide- $\mathrm{T}$ and $\mathrm{CaCl}_{2}$ treated conditions with the exception of $\Delta c n b 1$. Whereas $~ 75 \%$ of the cells showed nuclear localisation of Crz1p-GFP in $40 \mathrm{mM} \mathrm{ZnCl}_{2}$ treated conditions. 


\section{$\underline{\text { Discussion }}$}

5.18 Plakortide-T is a substrate for the PDR drug efflux pumps.

Plakortide-T was employed in dose response assays using pools of PD-DMA ( $\Delta p d r 1 \Delta p d r 3 \Delta x x x)$ and wild-type background DMA ( $\Delta x x x)$. Remarkably the deletion mutants in the wild-type genetic background showed no sensitivity to plakortide- $\mathrm{T}$ at the micromolar concentrations used (Fig. 5.2) whereas the PDR-deficient strains showed sensitivity to plakortide-T at nanomolar concentrations. Besides demonstrating that plakortide-T is a PDR substrate it has to be noted that its biological activity could not have been discerned in wild-type background screens. It is reasonable to conclude that the PD-DMA will be of general use as a primary screen in high throughput chemical genetic assays where the quantity of test compound is limited (as with natural products) or where targets may not be discernible at all because of the PDR drug efflux.

To identify the PDR pumps that were responsible for efflux of plakortide-T, dose response assays were performed on yeast mutant strains of the major PDR efflux transporters, $\Delta p d r 5, \Delta s n q 2, \Delta y o r 1$ and the minor and low affinity PDR efflux transporters, $\Delta p d r 10, \Delta p d r 11, \Delta p d r 12$ and $\Delta p d r 15$ against plakortide-T. The sensitivities of these drug efflux transporter mutants to plakortide-T was compared against the $\Delta p d r 1 \Delta p d r 3$ and the $\Delta h i s 3$ control strains. These PDR efflux transporter mutants showed no sensitivity to plakortide-T at any of the concentrations tested and was similar to the $\Delta h i s 3$ "wild-type" control (Fig. 5.5). Only the $\Delta p d r 1 \Delta p d r 3$ control strain showed sensitivity to plakortide-T. The PDR mediated efflux of plakortide-T is therefore likely to be the result from either one of the several other minor efflux transporters not screened in the dose response assays above or may involve multiple 
drug efflux transporters. If a single efflux transporter was responsible for the efflux of plakortide-T, then screening all the remaining efflux transporter mutants independently is not feasible as the expression of several hundred genes are directly regulated by Pdr1p and Pdr3p transcription factors $(40,86)$ and the amounts of plakortide-T available would not permit such a study. A competitive growth assay such as the DNA barcode microarray may identify deletion mutants defective in efflux of plakortide-T.

In the latter scenario, if multiple efflux transporters are involved in the efflux of plakortide-T, a gene knockout of a single efflux transporter may not have a significant effect on plakortide-T efflux. Therefore, combinations of several PDR pump mutants may be required to observe increased sensitivity to plakortide-T. Such studies would also be limited by the amounts of plakortide-T (or other natural product) required for the numerous dose response assays of numerous gene deletion combinations. Plakortide-T thus illustrates the need for deletion of the transcription factors of the PDR network rather than the individual efflux transporters to create an effective PDRdeficient strain. This affirms the approach in this thesis to construct a more drug sensitive yeast strain and the subsequent hypersensitive PD-DMA as a primary screening tool in drug discovery. 


\subsection{Plakortide-T is cytostatic}

In the initial dose response assays with plakortide-T (Fig. 5.2), complete inhibition was not observed for PDR-deficient strains, $\Delta p d r l \Delta p d r 3 \Delta x x x$ at the concentrations used in the dose response assay. In the $\Delta p d r 1 \Delta p d r 3 \Delta x x x$ strains a residual growth of $\sim 11 \%$ was observed across at the four highest concentrations spanning two orders of magnitude. This may have resulted from plakortide-T reversibly inhibiting growth of the PDRdeficient cells rather than killing cells. It was possible although highly unlikely that the growing cells were a small number of deletion mutants with wild-type background present among the PD-DMA which would be resistant plakortide-T as plakortide-T is inactive in wild-type yeast. Alternatively, some deletion mutants in the PDR-deficient background may also confer resistance to plakortide-T.

To test this latter possibility, dose response assays were performed on the $\Delta p d r 1 \Delta p d r 3$ and the wild-type yeast strains with each strain comprising a single type of mutant (Fig. 5.3). The $\Delta p d r 1 \Delta p d r 3$ strain as with $\Delta p d r 1 \Delta p d r 3 \Delta x x x$ did not show complete inhibition and also showed $\sim 11 \%$ residual growth at the highest concentrations of plakortide-T tested. Experiments described in Fig. 5.3 and Fig. 5.4 showed that plakortide-T reversibly inhibits the growth of PDR-deficient cells, thus, confirming plakortide-T as a cytostatic compound, a property that may help to explain the absence of complete inhibition following plakortide-T treatment in the PDR-deficient strains. 


\section{$\underline{5.20}$ Plakortide- $\mathrm{T}$ effects can be exacerbated by $\mathrm{ZnCl}_{\underline{2}}$ and $\mathrm{CaCl}_{\underline{2}}$.}

The elemental analysis of PFA treated cells showed an increase in both intracellular concentrations of $\mathrm{Ca}^{2+}$ and $\mathrm{Zn}^{2+}$, although, the increase in $\mathrm{Ca}^{2+}$ was significantly more in PFA treated cells (129). The quantification of possible increased intracellular calcium from plakortide- $T$ treatment was attempted using the long wavelength calcium indicator, calcium orange. Calcium orange was utilised in the current study as it was previously used effectively in studies involving mammalian cells and was available in the laboratory. No interpretable results were obtained from this assay (results not shown). It is possible that calcium orange may not work in S. cerevisiae and no other studies were found that employed calcium orange in S. cerevisiae. Nonetheless there does appear to be a calcium effect with plakortide- $\mathrm{T}$ since treatment $\mathrm{CaCl}_{2}$ and $\mathrm{ZnCl}_{2}$ increased the potency of plakortide- $\mathrm{T}$ in a non-additive manner (Fig. 5.6). Exacerbation of plakortide- $\mathrm{T}$ toxicity was greater with $\mathrm{Ca}^{2+}$ than $\mathrm{Zn}^{2+}$. Therefore, plakortide- $\mathrm{T}$ may induce growth inhibition by increasing either $\mathrm{Ca}^{2+}$ or $\mathrm{Zn}^{2+}$ levels although, the DNA barcode microarray failed to identify such a response. The $\mathrm{Ca}^{2+}$ homeostasis disruption by plakortide-T seems of more importance to its mechanism of action than $\mathrm{Zn}^{2+}$ and is consistent with what elemental analysis of PFA treated cells has previously shown (129).

5.21 Plakortide-T induced growth arrest could not be rescued by pre-incubating in $\underline{\text { EGTA }}$

A possible increase in cytosolic $\mathrm{Ca}^{2+}$ concentration contributed by the external environment via membrane bound $\mathrm{Ca}^{2+}$ pumps was investigated by pre-treating the PDR-deficient cells and the media with an $\sim \mathrm{IC}_{30}$ concentration of the $\mathrm{Ca}^{2+}$ chelator 
EGTA before adding plakortide-T (Fig. 5.7). The pre-treatment of PDR-deficient cells with EGTA did not reduce the plakortide-T induced growth inhibition. From these findings it cannot be concluded that plakortide- $\mathrm{T}$ does not cause an increase cytosolic $\mathrm{Ca}^{2+}$, rather it shows that little or no contribution is made by the extracellular $\mathrm{Ca}^{2+}$ to intracellular calcium levels via the plasma membrane $\mathrm{Ca}^{2+}$ transporters.

$\underline{5.22}$ Mutants in $\mathrm{Ca}^{2+}$ and $\mathrm{Zn}^{2+}$ transporters and calcineurin do not show increased sensitivity to plakortide-T.

The sensitivities of the $\mathrm{Ca}^{2+}$ and $\mathrm{Zn}^{2+}$ homeostasis mutants to plakortide- $\mathrm{T}$ was determined since the biological activity plakortide- $T$ was exacerbated by treatment with calcium chloride and zinc chloride and calcium homeostasis mutants also demonstrated increased sensitivity to PFA. In the dose response assays performed, $\mathrm{Ca}^{2+}$ and $\mathrm{Zn}^{2+}$ homeostasis mutants ( $\Delta v c x 1, \Delta p m c 1, \Delta p m r 1, \Delta c c h 1, \Delta m i d 1, \Delta y v c 1, \Delta e c m 7, \Delta z r t 1$, $\Delta z r t 3, \Delta z r t 2, \Delta z r c 1, \Delta c n b 1, \Delta c n a 1, \Delta c m p 2)$ in the PDR-deficient background showed different responses to $\mathrm{CaCl}_{2}, \mathrm{ZnCl}_{2}$ and plakortide-T. The $\Delta p m c 1$ mutant strain showed increased sensitivity to $\mathrm{CaCl}_{2}$ (Fig. 5.8a). $P M C l$ encodes for a calcium transporter which pumps $\mathrm{Ca}^{2+}$ from the cytosol to the Golgi which is the main intracellular calcium store in yeast where nearly $90 \%$ of cellular $\mathrm{Ca}^{2+}$ is immobilised (135). Therefore the $\Delta p m c 1$ mutant has permanently increased levels of cytosolic $\mathrm{Ca}^{2+}$ in comparison to the other $\mathrm{Ca}^{2+}$ homeostasis mutants; as such it is more sensitive to $\mathrm{CaCl}_{2}$. These findings were consistent with the literature as the $\Delta p m c 1$ mutant was previously identified as hypersensitive in media supplemented with $\mathrm{Ca}^{2+}(135)$. 
In the dose response assays using $\mathrm{ZnCl}_{2}$, the midl and $v c x l$ mutants showed better growth in media supplemented with non-inhibitory and semi inhibitory concentrations $\mathrm{ZnCl}_{2}$. This result was unexpected since $M I D 1$ encodes for the plasma membrane transporter which pumps $\mathrm{Ca}^{2+}$ to cytosol and $V C X 1$ encodes for a vacuolar transporter which pumps $\mathrm{Ca}^{2+}$ from the vacuole to cytosol both which contributes the transient increase in cytosolic $\mathrm{Ca}^{2+}$. It is puzzling why the deletions of these genes have reduced growth in SC media and better growth in SC media supplemented with $\mathrm{ZnCl}_{2}$ concentrations which is either non-inhibitory or semi-inhibitory in the $\Delta$ his 3 control (Fig. 5.8c).

However none of these findings with $\mathrm{CaCl}_{2}$ or $\mathrm{ZnCl}_{2}$ were observed for plakortide- $\mathrm{T}$ against the $\mathrm{Ca}^{2+}$ and $\mathrm{Zn}^{2+}$ homeostasis mutants as all the deletion mutants demonstrated similar sensitivities to the $\Delta$ his 3 control (Fig. 5.8d). This shows that the mechanism of action of plakortide- $\mathrm{T}$ is different from $\mathrm{Ca}^{2+}$ and $\mathrm{Zn}^{2+}$ toxicity. Likewise its action is different to PFA as none of the calcium homeostasis mutants were sensitive to plakortide-T.

\section{$\underline{5.23 \text { Deletion mutants hypersensitive to plakortide-T are not associated with calcium }}$} homeostasis disruption.

The sensitivity of the gene deletion strains to plakortide-T was assessed in a DNA barcode microarray experiment (Chapter 2 Materials and Methods) utilizing the haploid PDR-deficient mutants. The DNA barcode microarray experiment identified 55 phenotypic enhancements (PE) with plakortide-T of which 10 genes encoded proteins 
that interact with the mitochondrion (Table 5.3). No distinct biological processes were affected by plakortide-T as determined by GO analysis. The non-specific clustering of "hits" from the DNA barcode microarray as seen for plakortide-T may simply reflect a pleiotropic effect of the drug interfering with many unrelated targets or alternatively an interference with such a basic general pathway that no specific category of GO is enriched.

A recent study in S. cerevisiae using another cyclic peroxide isolated from Plakortis spp. of marine sponge, plakortide-F acid (PFA) was shown to inhibit growth by disrupting $\mathrm{Ca}^{2+}$ homeostasis (129). In this study, the mRNA expression profile of FPA treated cells closely resembled exposure to high levels of $\mathrm{CaCl}_{2}$ or amiodarone treatment and several deletion mutants of genes required for $\mathrm{Ca}^{2+}$ homeostasis, particularly, $\Delta p m r 1, \Delta p m c 1, \Delta m i d 1, \Delta c c h 1, \Delta c n a 1, \Delta c n b 1, \Delta c m p 2$ and $\Delta c r z 1$ demonstrated hyper sensitivity to PFA (129). Although none of these deletion mutants were among the strains hypersensitive to plakortide-T identified in the DNA barcode microarray it is still possible disruption of $\mathrm{Ca}^{2+}$ homeostasis by plakortide- $\mathrm{T}$ is occurring. Thus the deletion mutants hypersensitive to plakortide-T in the PDRdeficient background were compared against the 100 deletion strains most sensitive to amiodarone in Parsons et al. (65). Only two overlaps were observed between the two screens which included $A S I 1$, a gene encoding an integral member of the E3 ubiquitin ligase and TES1 encoding a peroxisomal acyl-CoA thioesterase (62). Therefore, plakortide-T, unlike plakortide-F acid, showed no functional similarity to amiodarone. Furthermore, from the PFA hypersensitive calcium homeostasis mutants ( $\Delta p m r 1$, $\Delta p m c 1, \Delta m i d 1, \Delta c c h 1, \Delta c n a 1, \Delta c n b 1, \Delta c m p 2$ and $\Delta c r z 1)$, only $\Delta c n b 1$ and $\Delta c r z 1$ mutants were present among the top 100 most sensitive strains to amiodarone (65). 
Therefore mechanism of PFA is not that similar to amiodarone. No genome-wide yeast deletion mutant screens have been published for $\mathrm{CaCl}_{2}$ and so this aspect of plakortideT could not be further compared.

The deletion mutants hypersensitive to plakortide- $\mathrm{T}$ were also compared against the 100 most sensitive deletion strains to $\mathrm{H}_{2} \mathrm{O}_{2}$ (65), since several strains defective in mitochondrial proteins were hypersensitive to plakortide- $T$, which is a possible indication of reactive oxygen species generation. However, the only commonality between the two data sets was $C Y C 1$ which encodes for cytochrome C (62). Therefore, the primary ROS generated by plakortide-T may not be $\mathrm{H}_{2} \mathrm{O}_{2}$ or that plakortide-T does not generate ROS.

\subsection{Plakortide-T does not generate ROS}

Possible generation of ROS and consequent apoptosis induction by plakortide-T was investigated using the GFP reporter strain, $\triangle p d r 1 \Delta p d r 3 Y A P 1-G F P$. The Yap1p showed exclusively cytoplasmic location under normal conditions and response to plakortide-T treatment (Fig. 5.9). This showed that plakortide-T does not induce the oxidative stress response detectable by nuclear localisation Yap1p. On the other hand, Yap1p ROS generation could be masked by a possible (but unobserved) activation of the calcium signalling pathway by plakortide-T. The activation of calcineurin has shown to dephosphorylate and inactivate Yap1p (144). Therefore, detection of ROS generation by plakortide-T was attempted by another approach which measured the oxidation of free thiols which is one of the main targets of ROS (138) (Fig. 5.10). Plakortide-T does 
not show increased oxidation of free thiols therefore it remains unlikely that plakortideT generates ROS as the DNA barcode microarray results here already suggested. However, oxidative stress response refers to different conditions specific to each type of ROS (138) as previously observed for menadione and $\mathrm{H}_{2} \mathrm{O}_{2}$ (140). Therefore different approaches to detect ROS generation by plakortide-T might be justifiable if in the unlikely event plakortide-T turns out to be a compelling drug lead.

\section{$\underline{5.25 \text { Reporters of increased cytosolic } \mathrm{Ca}^{2+} \text { and } \mathrm{Zn}^{2+}}$}

GFP reporter strains of calcium responsive transcription factors, CRZ1-GFP, HOG1GFP and MSN2-GFP and zinc responsive transcription factor, ZAP1-GFP were evaluated as possible reporters of increased cytosolic $\mathrm{Ca}^{2+}$ and increased cytosolic $\mathrm{Zn}^{2+}$, respectively (Fig. 5.11 \& Fig. 5.12). The Crz1p-GFP fusion protein was identified as an effective reporter for increased cytosolic $\mathrm{Ca}^{2+}$ whereas Hog1p-GFP and Msn2p-GFP were ineffective. Hog $1 \mathrm{p}$ was activated only slightly at highest concentration of $\mathrm{CaCl}_{2}$ tested whereas only a few cells showed activated Msn2 in response to $\mathrm{CaCl}_{2}$. This is consistent with a transient nuclear localisation of Hog1p and Msn2p observed by Takatsume et al. (2010) where nuclear localisation of Hog1p-GFP and Msn2p-GFP was immediate after addition of $300 \mathrm{mM} \mathrm{CaCl}_{2}$ and completely reversed to cytosolic location within 30-45 $\min$ (145). In the current work this response was not observed because the confocal microscopy was performed after $4 \mathrm{~h}$ incubation in each condition. However, Msn2p was activated in a few cells in the absence of $\mathrm{CaCl}_{2}$ treatment, which suggest activation of Msn2p by general stress mechanisms consistent with literature

$(62,141)$. In contrast, Crzlp is specifically activated by $\mathrm{Ca}^{2+} /$ calmodulin dependent activation of calcineurin and is a $\mathrm{Ca}^{2+}$ specific response $(134,145)$. 
The Crz1p-GFP fusion protein was also relocated to the nucleus by $\mathrm{ZnCl}_{2}$ treatment which was unexpected (Fig.5.12b). The nuclear re-localisation of Crz1p-GFP was shown not to be activated by general stress by treatment such as with $\sim \mathrm{IC}_{30}$ concentration of latrunculin-A. This suggests that Crz1p-GFP localisation was $\mathrm{Ca}^{2+}$ and $\mathrm{Zn}^{2+}$ specific response and not induced by general stress. Therefore there may yet be an unknown regulator of calmodulin/calcineurin pathway which is responsive to $\mathrm{Zn}^{2+}$. Alternatively, activation of Crzlp-GFP in response to $\mathrm{Zn}^{2+}$ could be related to a response to $\mathrm{Ca}^{2+}$ where an increase in cytosolic $\mathrm{Zn}^{2+}$ may be followed by an increase in cytosolic $\mathrm{Ca}^{2+}$.

The $\mathrm{Zn}^{2+}$ responsive transcription factor, Zaplp was employed as a reporter for increased $\mathrm{Zn}^{2+}$ concentration. The Zaplp-GFP fusion protein was expected to be cytoplasmic following $\mathrm{ZnCl}_{2}$ treatment since expression of $Z A P 1$ is repressed under zinc replete conditions (146). However, no downregulation of ZAP1 was observed with $\mathrm{ZnCl}_{2}$. Therefore, Zap1p was not an effective reporter for increase in cytosolic zinc.

The zinc transporter, Zrc1p, was also assessed as a reporter for increased intracellular zinc. $\mathrm{Zrc1p}$, transports $\mathrm{Zn}^{2+}$ from the cytosol to the vacuole for sequestration under $\mathrm{Zn}^{2+}$ replete conditions (146). Induction of Zrc1p-GFP was expected as over-expression of $Z R C l$ confers resistance to $\mathrm{Zn}^{2+}$ toxicity while its deletion increases sensitivity to $\mathrm{Zn}^{2+}$ (146). However, no change in $\mathrm{Zrc1p}$-GFP was observed with $\mathrm{ZnCl}_{2}$, and therefore was not an effective reporter for increased intracellular $\mathrm{Zn}^{2+}$ (Fig. 5.12c-d). 
5.26 Plakortide- $\mathrm{T}$ causes an increase in cytosolic $\mathrm{Ca}^{2+}$ concentration which requires calcineurin

Since a protein with a measurable change to an increased cytosolic $\mathrm{Zn}^{2+}$ was not found, Crzlp-GFP was used as the reporter both $\mathrm{Ca}^{2+}$ and $\mathrm{Zn}^{2+}$ homeostasis disruption. The $C R Z 1-G F P$ reporter in the $\Delta p d r 1 \Delta p d r 3$ background was treated with plakortide-T, $\mathrm{CaCl}_{2}$ and $\mathrm{ZnCl}_{2}$ (Fig. 5.13). Plakortide- $\mathrm{T}$ was shown to cause an increase in the concentration of either cytosolic $\mathrm{Ca}^{2+}$ or $\mathrm{Zn}^{2+}$, detected by the activation of Crz1p-GFP. $\mathrm{CaCl}_{2}$ and $\mathrm{ZnCl}_{2}$ was also shown to cause an increased in cytosolic $\mathrm{Ca}^{2+}$ and $\mathrm{Zn}^{2+}$ concentrations respectively in the CRZ1-GFP $\triangle p d r 1 \Delta p d r 3$ cells and was consistent with the CRZ1-GFP strain with the wild-type background .

The $\mathrm{Ca}^{2+}$ and $\mathrm{Zn}^{2+}$ homeostasis mutants in the CRZ1-GFP $\Delta p d r 1 \Delta p d r 3$ genetic background were treated with plakortide-T, $\mathrm{CaCl}_{2}$ and $\mathrm{ZnCl}_{2}$. These were compared against the $\Delta h i s 3 \Delta p d r 1 \Delta p d r 3 C R Z 1-G F P$ control strain (Fig. 5.14). Most mutants $\Delta x x x 1 \Delta p d r 1 \Delta p d r 3 C R Z 1-G F P$ behaved as the $\triangle h i s 3 \Delta p d r 1 \Delta p d r 3 C R Z 1-G F P$ control strain, the only exceptions being $\Delta p m r 1 \Delta p d r 1 \Delta p d r 3 C R Z 1-G F P$ and $\Delta c n b 1 \Delta p d r 1 \Delta p d r 3$ CRZ1-GFP. The $\triangle p m r 1$ mutant cells showed nuclear localisation of Crz1p-GFP across all conditions including the untreated control. This phenotype has not been observed or assessed previously in the literature. PMR1, encodes for a $\mathrm{Ca}^{2+}$ transporter which pumps $\mathrm{Ca}^{2+}$ from the cytosol to Golgi and ER (135). Therefore, the loss of PMR1 would lead to reduced ability of cells to sequester cytosolic $\mathrm{Ca}^{2+}$ leading to a permanently elevated cytosolic $\mathrm{Ca}^{2+}$ concentration compared to wild-type explaining why nuclear localisation of Crzlp-GFP was observed for the $\Delta p m r 1 \Delta p d r 1 \Delta p d r 3$ 
CRZ1-GFP mutant in untreated conditions. A similar response should also have been observed with the $\Delta p m c 1 \Delta p d r 1 \Delta p d r 3 C R Z 1-G F P$ mutant since Pmc1p pumps $\mathrm{Ca}^{2+}$ from the cytosol to the vacuole and vacuole is the main intracellular calcium store in yeast (135). However, Vcx1p also sequesters calcium in the vacuole therefore $\Delta p m c 1 \Delta p d r 1 \Delta p d r 3 C R Z 1-G F P$ mutant may sequester calcium using Vcx1p to reduce the cytoplasmic $\mathrm{Ca}^{2+}$ concentration below the threshold needed for activation of Crz1p.

In contrast the $\Delta c n b 1$ mutant cells did not activate Crzlp in response to plakortide-T, $\mathrm{CaCl}_{2}$ or $\mathrm{ZnCl}_{2}$. This was expected with $\mathrm{Ca}^{2+}$ since nuclear localisation of Crzlp requires calcineurin mediated dephosphorylation of cytosolic Crz1p, where the Cnb1p subunit is essential for calcineurin function (137). However, in response to $\mathrm{ZnCl}_{2}$, the $\Delta c n b 1$ mutant again showed no activation of Crz1p. This shows that Crzlp activation by increased cytosolic $\mathrm{Zn}^{2+}$ also requires calcineurin. Therefore, the likely conclusion will be that activation of Crzlp-GFP with $\mathrm{ZnCl}_{2}$ was a result of an initial increase in cytosolic $\mathrm{Zn}^{2+}$ leading to an increase in cytosolic $\mathrm{Ca}^{2+}$. The absence of Crz1p activation with plakortide- $T$ in the $\Delta c n b 1$ mutant shows that this response also requires calcineurin. Together this shows that the activation of Crzlp-GFP observed with plakortide-T treatment in the wild-type control was a result of an increase in cytosolic $\mathrm{Ca}^{2+}$ concentration. Therefore, plakortide- $\mathrm{T}$ induces calcium as a second messenger in it mechanism of action. However, from this experiment it is unclear whether it is solely an increase in cytosolic $\mathrm{Ca}^{2+}$ or an increase in cytosolic $\mathrm{Zn}^{2+}$ followed by an increase in cytosolic $\mathrm{Ca}^{2+}$ which leads to the activation of calcineurin and Crz1p. 


\subsection{Effects of calcineurin activation in the $\Delta p d r 1 \Delta p d r 3$ background}

It is possible that the mechanism of plakortide-T's bioactivity is directly related to deletions of PDR1 and PDR3. The Pdr1p/Pdr3p and Yap1p positively regulates the expression of RPN4, a key transcription factor of the ubiquitin proteasome system (80, 144). Yaplp has shown to be degraded by calcineurin activation (144). Since the activation calcineurin is consistent with plakortide-T treatment, it follows that Yap1p would be degraded. The degradation of Yap1p in the absence of Pdr1p and Pdr3p transcription factors may lead to a significant downregulation of RPN4 expression and the proteasome $(80,144)$. This can lead to an accumulation of proteins Swe1p and

$\mathrm{C} \ln 2 \mathrm{p}$ which negatively regulate entry into G2 phase of the cell cycle (144). A reduction in cell size by a delay in G2 was observed by Barber et al. with epiplakinic acid I (147), another 1, 2-dioxygenated cyclic peroxide metabolite from Plakortis spp. but no change in cell size was observed following treatment with plakortide-T. Therefore, it is unlikely that calcineurin activation is the mechanism of action of plakortide-T leading to growth arrest and must use some other pathway which is yet to be determined.

\section{$\underline{5.28 \text { Proposed mode of action of plakortide-T }}$}

Plakortide-T was identified as cytostatic in its mechanism of action as it reversibly inhibits growth in PDR-deficient cells. Plakortide-T belongs to a class of compounds known to disrupt calcium homeostasis. This was consistent with exacerbation of plakortide-T toxicity by calcium. The contribution to increased cytosolic calcium concentration by the external environment was assessed by pre-treating the media with 
a calcium chelator EGTA before treating with plakortide-T but no reduction in plakortide-T induced growth inhibition was observed.

The exacerbation of plakortide-T toxicity was also observed with zinc suggests a possible role for zinc in the mechanism of action of plakortide-T. The increased concentration of zinc in elemental analysis of PFA treated cells was also observed but was not investigated. However, plakortide-T hypersensitivity was not observed for neither calcium transport mutants, zinc transport mutants or to calcineurin mutants.

Therefore a DNA barcode microarray experiment was performed employing plakortideT. A number of Plakortide-T hypersensitive mutants were identified but failed to show functional enrichment by GO analysis. However, several genes encoding for mitochondrial proteins were identified. The generation of ROS as a possible mode of action of plakortide-T was investigated as mitochondria are one of the main targets of ROS but such a response was not confirmed as oxidation free thiols or induction of the oxidative stress response was not observed. Therefore the "Hits" showing mitochondrial localisation may represent an impairment of some other mitochondrial function.

The disruption of calcium homeostasis by plakortide- $\mathrm{T}$ was investigated further using Crz1p-GFP, a calcium responsive transcription factor. Plakortide-T caused nuclear localisation of Crz1p, which required functional calcineurin, as the $\Delta c n b 1$ mutant deficient for the regulatory subunit of calcineurin did not show this response. These findings validate the increased cytosolic calcium concentration caused by plakortide-T. 
However, there is not yet sufficient evidence to suggest that calcium homeostasis disruption is the cause of plakortide-T induced growth arrest. 


\section{Final discussion}

\subsection{The utility of the PDR-deficient strains and PD-DMA}

The PDR network presents one of the major difficulties in using Saccharomyces cerevisiae as a model organism in biochemical screens as it masks the effect of numerous compounds and attenuates the activity of several others by PDR network mediated drug efflux. This makes toxicity and target assays unfeasible. The MDR transporters in mammals and PDR in yeast uses different mechanisms for substrate recognition, therefore, a compound which shows low or no activity in yeast due to high affinity for PDR efflux transporters may be highly potent against mammalian cells. Compounds, camptothecin, idarubicin and diphenyleneiodonium chloride are some of PDR substrates that were exclusively bioactive in the PDR-deficient background from the LOPAC library these are also bioactive in mammalian cell (148). Therefore, such compounds might be missed from primary screens of chemicals in S. cerevisiae. The discussion that follows makes a strong case for the utility of the PDR-deficient strain in primary screens of novel compounds and PD-DMA in the elucidation of compound's targets.

The PDR-deficient strain constructed in this thesis causes an attenuation of the entire PDR response by disrupting the major transcription factors that control the PDR network, rather than deletion of individual efflux transporters which would only sensitise yeast substrates which utilises the specific transporters. Prime examples of this are latrunculin-A and plakortide-T. The loss of Pdr1p and Pdr3p transcription factors leads to significant reduction of $\operatorname{Pdr} 5 p$ (36), which was identified as the efflux transporter for latrunculin-A. As a consequence latrunculin-A was $\sim 28$ fold more 
sensitive in the $\Delta p d r 1 \Delta p d r 3$, PDR-deficient strain. Even more compelling, plakortide-T was only active in the PDR-deficient background. This sensitivity was most likely induced from transcriptional down regulation of multiple efflux transporters in the PDR-deficient background where several efflux transporters may collectively mediate the efflux of plakortide-T. Such biological activity of plakortide-T therefore, would not have been identified by using a simpler approach such as using single mutants of PDR pumps. However, the disruption of both PDR1 and PDR3 leads to transcriptional down regulation of efflux transporters under the control of $P D R 1$ and $P D R 3$ genes and as a consequence, lowers the effective concentration of a given PDR substrate needed to achieve growth inhibition. To achieve the same effective concentration within cells with wild-type PDR requires flooding the cellular machinery beyond its capacity to efflux. This may induce other undesired general stress mechanisms additional to the compound's mode of action, possibly masking true activities.

Another key point emerging from this thesis is that the absence of Pdr1p and Pdr3p does not induce or impair unfolded protein response or the cellular oxidative stress response. These characteristics of the PD-DMA are important for mode of action studies which in general are aimed at using the genome wide deletion set to identify a few target genes or pathways affected by a given compound without added complications such as stress responses. Adding to this point and the properties relating to general utility, no phenotypic enhancements were identified in the $\Delta p d r 1 \Delta p d r 3 \Delta x x x$, triple mutants, comprising the PD-DMA. This is a remarkable fact in its own right for on average 34 phenotypic enhancements are observed with pairwise deletions when using a single query gene and significantly more when generating triple mutants (94). Relating to the properties of the PD-DMA produced in this thesis, only 73 triple 
mutants were unable to be constructed during the SGA procedure as a result of mating deficiencies, sporulation deficiencies, extreme linkage disequilibrium and pinning errors therefore giving a near comprehensive coverage by the PD-DMA.

The PDR-deficient deletion mutant array allows a unique opportunity to study the mechanism of action of novel compounds including marine natural products, which are often initially available only in small quantities. Utilising chemo-genomic tools for dissecting molecular mechanisms of the bioactive compound in an unbiased manner may require screening against large numbers of deletion mutants, requiring significant amount of compound. Thus novel compounds available in small yields may be insufficient to do genome wide studies in conventional haploid and diploid DMA due to their reduced sensitivity. Therefore, a PD-DMA represents a major addition to the armamentarium of genomic screening tools. No such PD-DMA has been reported in the literature in to date.

The efficacy PDR-deficient strains were validated by examining sensitivity against the well-known compound cycloheximide, a Pdr5p substrate. The PD-DMA was validated in chemical genetic profiling assays against compounds cycloheximide and rapamycin that have known chemical-genetic profiles. In a solid-phase chemical genetic profiling assay employing rapamycin an inhibitor of TORC1 genes enriched for TORC1 pathway and other compensatory pathways to TORC1 as previously observed for the wild-type DMA. Similarly, in a barcode microarray using the PDR-deficient deletion mutant pools genes enriched for translational elongation with cycloheximide. 
The genome wide sensitivity of the PD-DMA was validated by screening the commercially available LOPAC library of compounds. Screening the LOPAC library using the conventional DMA pools identified five out of the 1280 compounds as having biological activity. In the same assay PD-DMA identified the same five compounds and 20 additional compounds to which the conventional DMA was insensitive at the concentration used. This highlights the increased sensitivity of the PDR-deficient mutants to compounds.

The PD-DMA was then utilised in a DNA barcode microarray to probe the activity of a less-well characterised marine natural product, latrunculin-A. This compound is known to bind monomeric actin to disrupt microfilament assembly purportedly without disrupting microtubule assembly (122). However, genes enriched in the chemogenomic profile for latrunculin-A included tubulin cytoskeleton genes as well the expected actin cytoskeleton genes. These "hits" were individually validated against latrunculin-A strengthening the evidence for their involvement in latrunculin-A activity. Microtubule disruption by latrunculin-A has not previously been reported in literature and probably could not have been because PDR-deficient strains were not used. This is mostly due to disruption of tubulin associated genes modifying actin associated pathways. This more detailed account of latrunculin-A activity highlights the utility of the PD-DMA as more drug sensitive and a more efficient DMA. 
Following validation of the PD-DMA as a more drug-sensitive DMA, as described in this thesis it has since been used in the research of seven other students in this department. Examples are the elucidation of the mechanisms of action of laulimalide, benomyl, FC592, the hemigerans, and TA-289. The targets of laulimalide a microtubule disrupting agent were discerned as processes involving tubulin dynamics since deletion mutants of tubulin associated genes including tubulin motors of kinesin and dynein showed hypersensitivity (149).

\section{$\underline{6.2 \text { Mode of action of plakortide-T }}$}

In this study using the PDR-deficient mutants several novel aspects of plakortide-T biological activity was observed. Plakortide-T was identified to reversibly inhibit growth and like other 1,2-dioxygenated cyclic peroxides, was shown to disrupt calcium homeostasis (129), however, this may not be the main mechanism of action as calcium transport and calcineurin mutants failed demonstrate hypersensitivity to plakortide-T. Several other compounds have also shown to disrupt calcium homeostasis. The most extensively studied example is amiodarone. However, the mechanism underlying the disruption of calcium homeostasis by amiodarone has not been determined (150).

The DNA barcode microarray experiment with plakortide-T showed hypersensitivity in mutants involved in various biological processes, which is apparent of a compound that may target a key gene or multiple genes. The former proposition is more likely as plakortide-T is structurally complex therefore, may not interact with multiple targets. The mitochondrial genes identified in the DNA barcode microarray were not because of 
generation of reactive oxygen species. This suggests a novel mechanism for growth inhibition by plakortide-T compared with other 1,2-dioxygenated cyclic peroxides.

\subsection{Limitations to the techniques used in this study}

Using chemical genetic profiling underplays the complexity of gene-product interactions (the proteome) where one gene may encode for more than one protein. A given drug may interact with only one or several variants of the proteins encoded by a single gene or genes. Therefore, deletion of a gene may not be equivalent to a loss of specific drug target but rather loss of the all protein variants encoded by that gene, where some protein variants may have different function to the drug target. The subsequent genetic interactions generated by a deletion of the compounds target may entirely not represent the true chemical genetic profile for its compound. Interpretation of "hits" in these types of screens must therefore take into account these possible outcomes.

A further limitation in this study was the use of the non-essential DMA in the construction of the PD-DMA by SGA methodology. As a consequence the essential genes were not interrogated to the PD-DMA. Therefore, the complete set of chemicalgenetic interactions to a compound of interest cannot be observed. Furthermore, the DNA microarray technology was used to limit drug use. The DNA barcode microarray identifies the fitness of the deletion mutants indirectly by hybridisation of barcodes denoting the deletion mutants to oligos on a microarray chip. Nearly 1000 genes in each barcode microarray experiment did not hybridise as multiple steps are involved from 
initial compound treatment up the hybridisation step. This is due to the multiple steps involved DNA barcode microarray several barcodes are lost along the way. It has been recently published that up to $20 \%$ of the common primer sites and barcodes denoting the specific deletion mutations carry point mutations (128). Although, this is probably ameliorated by using both UP and DN tags, the sensitivity profiles of several mutants may be compromised in each barcode microarray experiment. This can be improved by sequencing of barcodes rather hybridising to a microarrays, an increasingly attractive and feasible option as the cost of sequencing continues to decrease.

A further limitation in the use of microarrays is that at times they yield uninterruptable results, which was evident from the DNA barcode microarray employing plakortide-T. This is also a consequence of measuring the fitness of the deletion mutants indirectly, where the fitness of the deletion mutants are identified by hybridisation of barcodes denoting the deletion mutants. An overall reduced level of PCR products in either from the control or compound treated conditions can offset the balance (the Cy5/Cy3 ratio) used to identify mutants fitness. This and the non-specific hybridisation barcodes may generate noise that will mask the true sensitivities of the deletion mutants. Chemical genetic profiling can also yield uninterruptable results when dealing with compound targets that affect multiple pathways.

Another possible limitation in using the PDR-deficient strains is that several genes are controlled by PDRI and Pdr3p transcription factors including genes encoding for several membrane transporters which pump nutrients into the cell. The compounds that resemble nutrients may utilise these transporters for cell entry (77), and may show 
reduced sensitivity to the PDR-deficient strains due to the down regulation of these transporters by the loss of Pdr1p and Pdr3p transcription factors (31). Out of the 25 compounds in the LOPAC library identified as bioactive with the screens described in this thesis, dequalinium dichloride showed a slight yet a significant increase in resistance in the PDR-deficient strains in comparison to the wild-type background strains. The PDR-deficient strains showed increased sensitivity to 21 compounds where 20 were biologically inactive in the wild-type strains at the concentrations used. These 20 compounds can only be studied in the PDR-deficient strains and their drug targets may potentially be elucidated by using PD-DMA. The PDR-deficient strains gives access to more compounds than the previously possible in preliminary chemical and high throughput screens and more opportunities to find potential therapeutics. This show cases the utility of the PDR-deficient strains and the PD-DMA. 


\section{$\underline{\text { 7. Future directions }}$}

The aim of the thesis was to construct and validate the suitability of a PDR-deficient deletion library to perform chemical genetic profiling studies on compounds that are targeted by the PDR network for efflux. This aim was achieved as the PDR-deficient strains demonstrated increased sensitivity to compounds that are PDR substrates and unaltered sensitivity to non-PDR substrates. The PD-DMA was validated in several studies in both solid and liquid media to establish the mode of action of both PDR substrates and non-PDR substrates. For example, disruption of tubulin-related gene increased sensitivity latrunculin-A which has not been previously reported (Chapter 4.6). Furthermore, the PD-DMA strains did not display induced or impaired unfolded protein response, or the oxidative stress response, allowing the interpretation of chemical genetic profiling without complication by these stress responses.

\subsection{The importance of plakortide-T drug target elucidation and identification}

The target of plakortide-T is highly conserved in eukaryotes as it showed potent biological activity in both PDR-deficient budding yeast and in mammalian cell lines (147). Plakortide-T is a 1,2-dioxygenated cyclic peroxide, these compounds are of particular interest due to their diverse pharmacological activities. These include; antifungal, antibacterial, anti-protozoal activity against several major human parasites and potent anti-tumour activity against murine and human cancer cell lines (129-131). Therefore elucidating their mechanism of action will greatly benefit the pharmacological field as current second and third line therapeutic drugs are fast becoming ineffective due to evolution of multi-drug resistance and extensive drug resistant pathogens and neoplasms (6). The elucidation of the mechanism of plakortide- 
T's bioactivity in this thesis was limited by the availability of compound. An important future direction will be to establish the target(s) of this compound should more become available.

\subsection{Future studies for elucidating the mechanism of action of plakortide-T}

\subsubsection{Resistant mutant generation and identification of the resistance mutation(s)}

It is possible to identify a compound's target by identifying genes that are resistant to the particular compound. Therefore, generation of resistant mutants in the $\Delta p d r 1 \Delta p d r 3$ strain is a potential next step if compound availability permits. These mutants will then be assessed for dominant and recessive nature of the resistant mutation. The genetic basis of the resistance mutation will be characterised using genome-wide linkage mapping technology, $\operatorname{SGAM}(151,152)$ to in hope to identify the drug target of plakortide-T.

\section{$\underline{7.2 .2 \text { Sensitivity of rho }(0) \text { mutants to plakortide-T }}$}

Although generation of reactive oxygen species was not observed with plakortide-T, the mitochondrial genes identified by the DNA barcode microarray may be due to impairment of some other mitochondrial function. Effects of plakortide- $T$ on mitochondrial function can be detected by using yeast rho (0) mutants which lack mitochondria, therefore such mutants will show increased resistance to plakortide-T. The activity of plakortide-T on mitochondria can be discerned using the yeast rho (0) mutants. 


\subsubsection{Detecting an increase in cytosolic $\mathrm{Zn}^{2+}$.}

Although, the nuclear localisation of Crzlp was consistent with an increase in cytosolic calcium it could not be determined whether it was calcium alone or an increase in zinc leading to an increase in cytosolic calcium leading to the response. The FRET system uses $\mathrm{ZF} 1$ and $\mathrm{ZF} 2$ zinc finger interacting pairs from Zap1p linked to a yellow fluorescent protein and a cyan fluorescent protein respectively. Binding of $\mathrm{Zn}^{2+}$ coordinates the $\mathrm{ZF} 1 / \mathrm{ZF} 2$ bring them in close proximity which can be detected by change in emission (153). An increase in cytosolic $\mathrm{Zn}^{2+}$ concentration in response to plakortide-T treatment can be detected using this system.

\subsubsection{Unfolded protein response (UPR) induction assay}

The influx of calcium from the external environment via Mid1p and Cch1p lead to depletion of several internal calcium stores including the ER (134). The depletion of ER calcium has been shown to interfere with the processing of secretory proteins and protein folding because calcium is required to coordinate protein folding (154). Therefore, depletion of ER calcium leads to an increase in protein misfolding which in turn leads to an induction of the UPR. The induction of the UPR can be detected by using the $\triangle p d r l \Delta p d r 3$ UPRE-GFP (constructed in Chapter 3.10) in confocal microscopy under plakortide-T treatment. 


\subsection{Future applications for the PD-DMA}

In this thesis several compensatory pathways additional to the compounds proposed mechanism of action were identified using the PD-DMA that were not observed with the conventional DMA (for example, disruption of the tubulin cytoskeleton by latrunculin-A, a novel finding of this thesis). The use of the PD-DMA allows the use of lower concentrations of compounds that are PDR substrates and in addition it also removes the need to flood the cellular machinery with compound to overcome the efflux mechanism to achieve growth inhibition. The flooding of cellular machinery with compound can lead to activation of stress responses which can make the compounds true activity less apparent. Therefore, the PD-DMA is more effective in elucidating the mode of action of PDR substrates by chemical genetic profiling. This sets the precedence for re-profiling previously profiled compounds using the PD-DMA to identify other pathways that may have not been identified using the in the wild-type DMA.

The findings from re-profiling in the PD-DMA may contribute towards the recent developments in pharmacology where the focus is moving from compounds that have a single target to using compounds that have multiple targets (155). It is now apparent that most cellular processes are regulated by several key genes and these genes cannot be targeted with compounds without having adverse effects on normal cellular function. Targeting a downstream gene product may have no effect of consequence due to functional redundancy of genes and pathways. The recent approach looks to perturb function of proteins at several positions in pathways to achieve the desired effects without disrupting essential cellular processes (155). This can also be achieved by using 
combinations of compounds where each compound target either different pathways or different genes with in the same pathway.

Several combination of drugs are already in use which include Advair for treatment of asthma, Advicor for treatment of hypercholesterolemia and Combivir for treatment of HIV (155). From these, Advicor is a combination of niacin and statin both of which target HMG-CoA and was developed on the based on the benefits of both compounds, where the combination leads to a greater decrease in low density lipoprotein and a greater increase in high density lipoprotein both of which are desired effects (155) . Therefore, generating a more complete set of compound target interaction will improve the understanding of the compound mechanism of action as well as giving insight in to possible compound combinations that can be used to target multiple proteins. This will be particularly useful in treating complex diseases where one target does not exist. In that respect latrunculin-A could be used in combination with either an actin cytoskeleton disrupting agents or with a microtubule disrupting agents as a muticomponent therapeutic, since latrunculin A has shown to affect both actin and tubulin associated proteins. 


\section{$\underline{\text { Appendix }}$}

Appendix 1
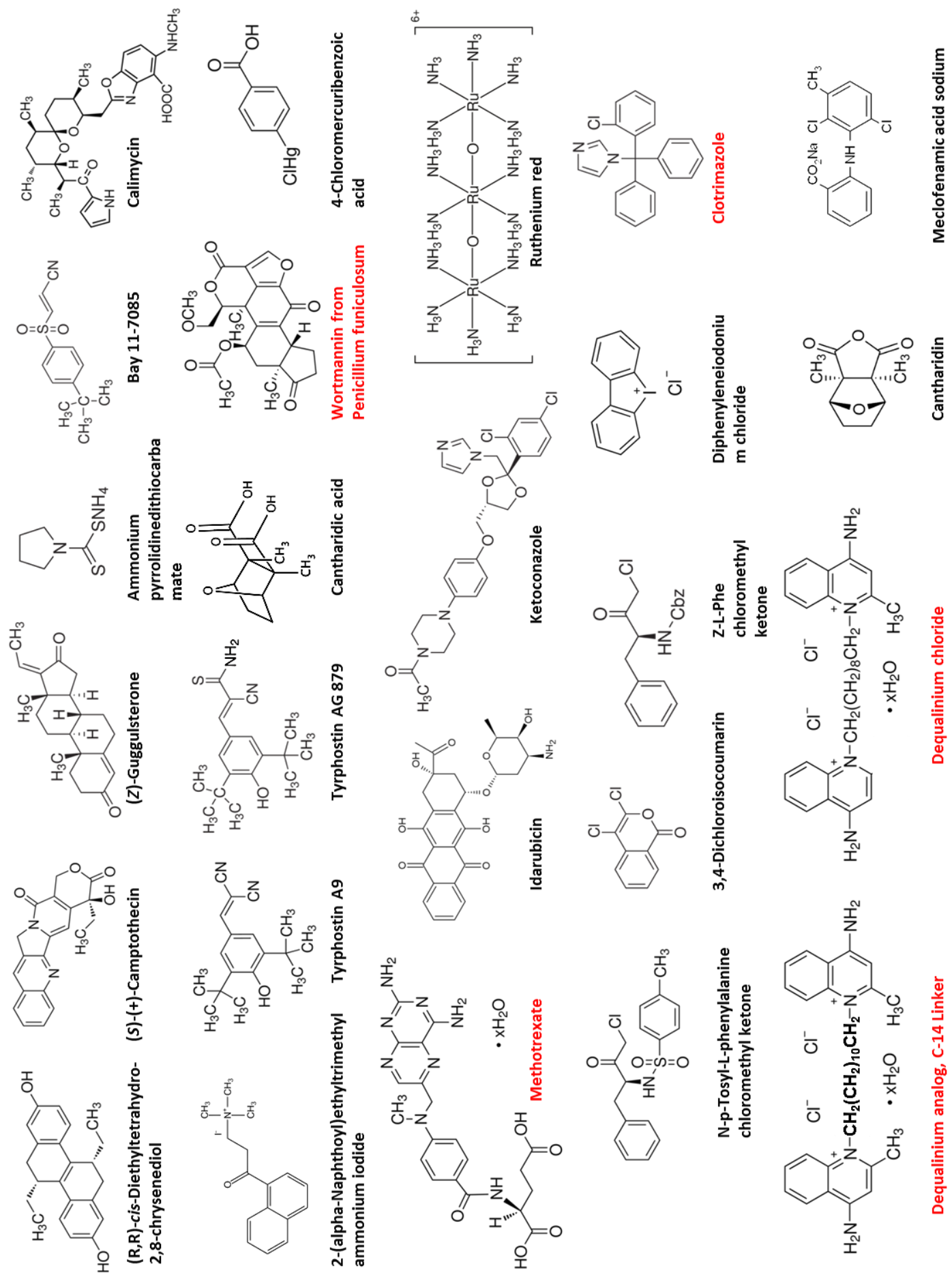

Figure 8.1 - Structures of LOPAC library compounds that showed biological activity in yeast.

Labelled in black are compounds that showed bioactivity only in the PDR-deficient strains. Labelled in red are compounds that are active in both wild-type and PDR-deficient strains. 
Appendix2

\begin{tabular}{|c|c|c|}
\hline ORF & Gene & Description \\
\hline YHR129C & ARP1 & Actin-related protein of the dynactin complex \\
\hline YMR119W & ASI1 & Putative integral membrane E3 ubiquitin ligase \\
\hline YJLO20C & BBC1 & Protein possibly involved in assembly of actin patches \\
\hline YIL159W & BNR1 & Formin \\
\hline YER048C & CAJ1 & Nuclear type II J heat shock protein of the E. coli dnaJ family \\
\hline YKL007W & CAP1 & Alpha subunit of the capping protein $(\mathrm{CP})$ heterodimer \\
\hline YIL034C & CAP2 & Beta subunit of the capping protein $(\mathrm{CP})$ heterodimer \\
\hline YMR198W & CIK1 & Kinesin-associated protein \\
\hline YIL035C & CKA1 & Alpha catalytic subunit of casein kinase 2 (CK2) \\
\hline YOR039W & CKB2 & Beta' regulatory subunit of casein kinase 2 (CK2) \\
\hline YIL157C & COA1 & $\begin{array}{l}\text { Mitochondrial membrane protein required for assembly of complex } \\
\text { IV }\end{array}$ \\
\hline YJR048W & CYC1 & Cytochrome c \\
\hline YAL026C & DRS2 & Trans-golgi network aminophospholipid translocase (flippase) \\
\hline YMR202W & ERG2 & C-8 sterol isomerase \\
\hline YCR034W & FEN1 & Fatty acid elongase \\
\hline YHL031C & GOS1 & v-SNARE protein involved in Golgi transport \\
\hline YNL281W & $\mathrm{HCH} 1$ & Heat shock protein regulator that binds to Hsp90p \\
\hline YIL116W & HIS5 & Histidinol-phosphate aminotransferase \\
\hline YGL168W & HUR1 & Protein of unknown function \\
\hline YDR123C & INO2 & Component of the Ino2p/Ino4p transcription activator \\
\hline YGL216W & KIP3 & $\begin{array}{l}\text { Kinesin-related motor protein involved in mitotic spindle } \\
\text { positioning }\end{array}$ \\
\hline YLL049W & LDB18 & Component of the dynactin complex \\
\hline YNL323W & LEM3 & Membrane protein of the plasma membrane and ER \\
\hline YOR231W & MKK1 & MAPKK involved in the protein kinase $\mathrm{C}$ signaling pathway \\
\hline YNL076W & MKS1 & Pleiotropic negative transcriptional regulator \\
\hline YNL099C & OCA1 & Putative protein tyrosine phosphatase \\
\hline YLR338W & OPI9 & Dubious open reading frame unlikely to encode a functional protein \\
\hline YGR078C & PAC10 & Part of the heteromeric co-chaperone GimC/prefoldin complex \\
\hline YOR153W & PDR5 & Plasma membrane ATP-binding cassette $(A B C)$ transporter \\
\hline
\end{tabular}




\begin{tabular}{|c|c|c|}
\hline YGL045W & RIM8 & Protein involved in proteolytic activation of Rim101p \\
\hline YOL039W & RPP2A & Ribosomal protein P2 alpha \\
\hline YDL225W & SHS1 & Component of the septin ring that is required for cytokinesis \\
\hline YDR011W & SNQ2 & Plasma membrane ATP-binding cassette (ABC) transporter \\
\hline YIL073C & SPO22 & Meiosis-specific protein essential for chromosome synapsis \\
\hline YDR463W & STP1 & Transcription factor \\
\hline YER111C & SWI4 & DNA binding component of the SBF complex (Swi4p-Swi6p) \\
\hline YJL004C & SYS1 & Integral membrane protein of the Golgi required for targeting Arl3p \\
\hline YBL054W & TOD6 & $\begin{array}{l}\text { PAC motif binding protein involved in rRNA and ribosome } \\
\text { biogenesis }\end{array}$ \\
\hline YNL079C & TPM1 & Major isoform of tropomyosin \\
\hline YDR074W & TPS2 & $\begin{array}{l}\text { Phosphatase subunit of the trehalose-6-phosphate synthase } \\
\text { complex }\end{array}$ \\
\hline YNL054W & VAC7 & $\begin{array}{l}\text { Integral vacuolar membrane protein involved in vacuole } \\
\text { organization }\end{array}$ \\
\hline YDR247W & VHS1 & Cytoplasmic serine/threonine protein kinase \\
\hline YKR001C & VPS1 & Dynamin-like GTPase required for vacuolar sorting \\
\hline YLR200W & YKE2 & Subunit of the heterohexameric Gim/prefoldin protein complex \\
\hline YBR178W & YBR178W & Dubious open reading frame unlikely to encode a functional protein \\
\hline YFR016C & YFR016C & Putative protein of unknown function \\
\hline YGL042C & YGL042C & Dubious open reading frame unlikely to encode a functional protein \\
\hline YIR042C & YIR042C & Putative protein of unknown function \\
\hline $\begin{array}{l}\text { YMR316C- } \\
\text { A }\end{array}$ & $\begin{array}{l}\text { YMR316C- } \\
\text { A }\end{array}$ & Protein of unknown function \\
\hline YNL120C & YNL120C & Dubious open reading frame unlikely to encode a functional protein \\
\hline YOR019W & YOR019W & Protein of unknown function \\
\hline
\end{tabular}

Table 8.1 - The complete List of genes deletion mutants hypersensitive to $28 \mathrm{nM}$ latrunculinA in a DNA barcode microarray experiment. 51 phenotypic enhancements were identified from a DNA barcode microarray utilizing the haploid PDR-deficient mutants. Genes were annotated according to SGD (62). 


\section{$\underline{\text { List of references }}$}

1. Fabricant DS \& Farnsworth NR (2001) The value of plants used in traditional medicine for drug discovery. Environmental Health Perspectives 109:69-75.

2. Li JW-H \& Vederas JC (2009) Drug Discovery and Natural Products: End of an Era or an Endless Frontier? Science 325(5937):161-165.

3. Wrigley SK \& Chicarelli-Robinson MI (1997) Chapter 29. Natural Products Research and Pharmaceuticals in the 1990's. Annual Reports in Medicinal Chemistry, ed James AB (Academic Press), Vol Volume 32, pp 285-294.

4. Lam KS (2007) New aspects of natural products in drug discovery. Trends in Microbiology 15(6):279-289.

5. Koehn FE \& Carter GT (2005) The evolving role of natural products in drug discovery. Nat Rev Drug Discov 4(3):206-220.

6. Fischbach MA \& Walsh CT (2009) Antibiotics for Emerging Pathogens. Science 325(5944):1089-1093.

7. Walsh DP \& Chang Y-T (2006) Chemical genetics. Chemical Reviews 106(6):2476-2530

8. Forsburg SL (2001) The art and design of genetic screens: yeast. Nat Rev Genet 2(9):659-668.

9. Simon JA \& Bedalov A (2004) Opinion - Yeast as a model system for anticancer drug discovery. Nat. Rev. Cancer 4(6):481-488.

10. Goffeau A, et al. (1996) Life with 6000 genes. Science 274(5287):546-\&.

11. Tong AHY, et al. (2001) Systematic genetic analysis with ordered arrays of yeast deletion mutants. Science 294(5550):2364-2368.

12. Gitler AD (2008) Beer and Bread to Brains and Beyond: Can Yeast Cells Teach Us about Neurodegenerative Disease? Neurosignals 16(1):52-62. 
13. Botstein D \& Fink GR (2011) Yeast: An Experimental Organism for 21st Century Biology. Genetics 189(3):695-704.

14. Sturgeon CM, Kemmer D, Anderson HJ, \& Roberge M (2006) Yeast as a tool to uncover the cellular targets of drugs. Biotechnology Journal 1(3):289-298.

15. Ho CH, et al. (2011) Combining functional genomics and chemical biology to identify targets of bioactive compounds. Current Opinion in Chemical Biology 15(1):66-78.

16. Winzeler EA, et al. (1999) Functional Characterization of the S.\&nbsp;cerevisiae Genome by Gene Deletion and Parallel Analysis. Science 285(5429):901-906.

17. Boone C, Bussey H, \& Andrews BJ (2007) Exploring genetic interactions and networks with yeast. Nature Reviews Genetics 8(6):437-449.

18. Giaever G, et al. (2002) Functional profiling of the Saccharomyces cerevisiae genome. Nature 418(6896):387-391.

19. Enserink JM (2012) Chemical Genetics: Budding Yeast as a Platform for Drug Discovery and Mapping of Genetic Pathways. Molecules 17(8):9258-9273.

20. Tashiro E \& Imoto M (2012) Target identification of bioactive compounds. Bioorganic \& Medicinal Chemistry 20(6):1910-1921.

21. Das RK, et al. (2011) Target Identification : A Challenging Step in Forward Chemical Genetics. IBC 3:3.

22. Cong F, Cheung AK, \& Huang S-MA (2012) Chemical Genetics-Based Target Identification in Drug Discovery. Annual Review of Pharmacology and Toxicology, Vol 52, Annual Review of Pharmacology and Toxicology, eds Insel PA, Amara SG, \& Blaschke TF), Vol 52, pp 57-78. 
23. Kley N (2004) Chemical Dimerizers and Three-Hybrid Systems: Scanning the Proteome for Targets of Organic Small Molecules. Chemistry \&amp; Biology 11(5):599-608.

24. Licitra EJ \& Liu JO (1996) A three-hybrid system for detecting small ligandprotein receptor interactions. Proceedings of the National Academy of Sciences 93(23):12817-12821.

25. Takahashi TT, Austin RJ, \& Roberts RW (2003) mRNA display: ligand discovery, interaction analysis and beyond. Trends in Biochemical Sciences 28(3):159-165.

26. O' Connor CJ, Laraia L, \& Spring DR (2011) Chemical genetics. Chemical Society Reviews 40(8):4332-4345.

27. Parsons AB, et al. (2004) Integration of chemical-genetic and genetic interaction data links bioactive compounds to cellular target pathways. Nature Biotechnology 22(1):62-69.

28. Heitman J, Movva NR, \& Hall MN (1991) Targets for cell-cycle arrest by the immunosuppressant rapamycin in yeast. Science 253(5022):905-909.

29. Gulshan K \& Moye-Rowley WS (2007) Multidrug resistance in fungi. Eukaryotic Cell 6(11):1933-1942.

30. Jungwirth H \& Kuchler K (2006) Yeast ABC transporters - A tale of sex, stress, drugs and aging. Febs Letters 580(4):1131-1138.

31. Bauer BE, Wolfger H, \& Kuchler K (1999) Inventory and function of yeast $\mathrm{ABC}$ proteins: about sex, stress, pleiotropic drug and heavy metal resistance. Biochimica et Biophysica Acta (BBA) - Biomembranes 1461(2):217-236.

32. Tegos GP, et al. (2011) Microbial Efflux Pump Inhibition: Tactics and Strategies. Current Pharmaceutical Design 17(13):1291-1302. 
33. Egner R, Bauer BE, \& Kuchler K (2000) The transmembrane domain 10 of the yeast Pdr5p ABC antifungal efflux pump determines both substrate specificity and inhibitor susceptibility. Molecular Microbiology 35(5):1255-1263.

34. Kolaczkowska A \& Goffeau A (1999) Regulation of pleiotropic drug resistance in yeast. Drug Resistance Updates 2(6):403-414.

35. Wolfger H, Mamnun YM, \& Kuchler K (2004) The yeast Pdr15p ATP-binding cassette $(\mathrm{ABC})$ protein is a general stress response factor implicated in cellular detoxification. Journal of Biological Chemistry 279(12):11593-11599.

36. Wolfger H, Mahe Y, ParleMcDermott A, Delahodde A, \& Kuchler K (1997) The yeast ATP binding cassette (ABC) protein genes PDR10 and PDR15 are novel targets for the Pdr1 and Pdr3 transcriptional regulators. Febs Letters 418(3):269-274.

37. Kolaczkowska A, Kolaczkowski M, Goffeau A, \& Moye-Rowley WS (2008) Compensatory activation of the multidrug transporters Pdr5p, Snq2p, and Yor1p by Pdr1p in Saccharomyces cerevisiae. Febs Letters 582(6):977-983.

38. Mamnun YM, Pandjaitan R, Mahé Y, Delahodde A, \& Kuchler K (2002) The yeast zinc finger regulators Pdr1p and Pdr3p control pleiotropic drug resistance (PDR) as homo- and heterodimers in vivo. Molecular Microbiology 46(5):14291440.

39. Hallstrom TC, et al. (2001) Coordinate control of sphingolipid biosynthesis and multidrug resistance in Saccharomyces cerevisiae. J. Biol. Chem.:M101568200.

40. DeRisi J, et al. (2000) Genome microarray analysis of transcriptional activation in multidrug resistance yeast mutants. Febs Letters 470(2):156-160.

41. Teixeira MC, Dias PJ, Simoes T, \& Sa-Correia I (2008) Yeast adaptation to mancozeb involves the up-regulation of FLR1 under the coordinate control of 
Yap1, Rpn4, Pdr3, and Yrr1. Biochemical and Biophysical Research Communications 367(2):249-255.

42. Gottesman MM \& Pastan I (1993) Biochemistry of multidrug-resistance mediated by the multidrug transporter. Annu. Rev. Biochem. 62:385-427.

43. Ernst R, Kueppers P, Stindt J, Kuchler K, \& Schmitt L (2010) Multidrug efflux pumps: Substrate selection in ATP-binding cassette multidrug efflux pumps first come, first served? FEBS Journal 277(3):540-549.

44. Ernst R, Klemm R, Schmitt L, \& Kuchler K (2005) Yeast ATP - Binding Cassette Transporters: Cellular Cleaning Pumps. Methods in Enzymology, eds Helmut S \& Lester P (Academic Press), Vol Volume 400, pp 460-484.

45. Golin J, Barkatt A, Cronin S, Eng G, \& May L (2000) Chemical Specificity of the PDR5Multidrug Resistance Gene Product of Saccharomyces cerevisiae Based on Studies with Tri-n-Alkyltin Chlorides. Antimicrobial Agents and Chemotherapy 44(1):134-138.

46. Golin J, et al. (2003) Studies with Novel Pdr5p Substrates Demonstrate a Strong Size Dependence for Xenobiotic Efflux. Journal of Biological Chemistry 278(8):5963-5969.

47. Rogers B, et al. (2001) The pleitropic drug ABC transporters from Saccharomyces cerevisiae. Journal of Molecular Microbiology and Biotechnology 3(2):207-214.

48. Golin J, Ambudkar SV, \& May L (2007) The yeast Pdr5p multidrug transporter: How does it recognize so many substrates? Biochemical and Biophysical Research Communications 356(1):1-5.

49. Emerson LR, Skillman BC, Wolfger H, Kuchler K, \& Wirth DF (2004) The sensitivities of yeast strains deficient in PDR ABC transporters, to quinoline- 
ring antimalarial drugs. Annals of Tropical Medicine and Parasitology 98:643649.

50. Pannunzio VG, et al. (2004) A Simple Chemical Method for Rendering WildType Yeast Permeable to Brefeldin A That Does Not Require the Presence of an erg6 Mutation. Journal of Biomedicine and Biotechnology 2004(3):150-155.

51. Tong AHY \& Boone C (2006) Synthetic genetic array analysis in Saccharomyces cerevisiae. Methods Mol Biol 313:171-192.

52. Tong AHY, et al. (2004) Global mapping of the yeast genetic interaction network. Science 303(5659):808-813.

53. Schüller C, et al. (2007) Membrane-active Compounds Activate the Transcription Factors Pdr1 and Pdr3 Connecting Pleiotropic Drug Resistance and Membrane Lipid Homeostasis in Saccharomyces cerevisiae. Molecular Biology of the Cell 18(12):4932-4944.

54. Brachmann BC, et al. (1998) Designer deletion strains derived from Saccharomyces cerevisiae S288C: A useful set of strains and plasmids for PCRmediated gene disruption and other applications. Yeast 14(2):115-132.

55. Tong AHY \& Boone C (2007) 16 High-Throughput Strain Construction and Systematic Synthetic Lethal Screening in. Methods in Microbiology, eds Ian S \& Michael JRS (Academic Press), Vol Volume 36, pp 369-386, 706-707.

56. Werner W (2005) A New Selection System for Molecular Biological Studies:

clonNAT + Plasmids pHN15 or pYL16.

57. Janke C, et al. (2004) A versatile toolbox for PCR-based tagging of yeast genes: new fluorescent proteins, more markers and promoter substitution cassettes. Yeast 21(11):947-962. 
58. Gietz RD \& Schiestl RH (2007) High-efficiency yeast transformation using the LiAc/SS carrier DNA/PEG method. Nat. Protocols 2(1):31-34.

59. Amberg DC, Burke D, Strathern JN, \& Laboratory CSH (2005) Methods in Yeast Genetics: A Cold Spring Harbor Laboratory Course Manual (Cold Spring Harbor Laboratory Press).

60. Dosil M, Giot L, Davis C, \& Konopka JB (1998) Dominant-Negative Mutations in the G-Protein-Coupled alpha -Factor Receptor Map to the Extracellular Ends of the Transmembrane Segments. Mol. Cell. Biol. 18(10):5981-5991.

61. Collins S, Schuldiner M, Krogan N, \& Weissman J (2006) A strategy for extracting and analyzing large-scale quantitative epistatic interaction data. Genome Biology 7(7):R63.

62. SGD (2010) Saccharomyces Genome Database.

63. Tong AH \& Boone C (2005) Synthetic Genetic Array Analysis in Saccharomyces cerevisiae. Yeast Protocols, (Humana Press), 2 Ed Vol 313, pp 171-191.

64. Cook MA (2007) Yeast Barcode Microarray Protocol. ed Bellows DB (Toronto, Ontario, M5G 1X5, Canada).

65. Parsons AB, et al. (2006) Exploring the Mode-of-Action of Bioactive Compounds by Chemical-Genetic Profiling in Yeast. Cell 126(3):611-625.

66. Colantuoni C, Henry G, Zeger S, \& Pevsner J (2002) Local mean normalization of microarray element signal intensities across an array surface: quality control and correction of spatially systematic artifacts. BioTechniques 32(6):1316-1320.

67. Cline MS, et al. (2007) Integration of biological networks and gene expression data using Cytoscape. Nat. Protocols 2(10):2366-2382. 
68. Maere S, Heymans K, \& Kuiper M (2005) BiNGO: a Cytoscape plugin to assess overrepresentation of Gene Ontology categories in Biological Networks. Bioinformatics 21(16):3448-3449.

69. Ashburner M, et al. (2000) Gene Ontology: tool for the unification of biology. Nat Genet 25(1):25-29.

70. Bircham PW, et al. (2011) Secretory pathway genes assessed by highthroughput microscopy and synthetic genetic array analysis. Molecular BioSystems 7(9):2589-2598.

71. Leberer E, Dignard D, Harcus D, Thomas DY, \& Whiteway M (1992) The protein kinase homologue Ste20p is required to link the yeast pheromone response G-protein beta gamma subunits to downstream signalling components. EMBO J 11(13):4815-4824.

72. Valtz N, Peter M, \& Herskowitz I (1995) FAR1 is required for oriented polarization of yeast cells in response to mating pheromones. The Journal of Cell Biology 131(4):863-873.

73. Chen P, Sapperstein SK, Choi JD, \& Michaelis S (1997) Biogenesis of the Saccharomyces cerevisiae Mating Pheromone a-Factor. The Journal of Cell Biology 136(2):251-269.

74. Yan G, Lai Y, \& Jiang Y (2012) The TOR Complex 1 Is a Direct Target of Rho1 GTPase. Molecular Cell 45(6):743-753.

75. Cherry JM, et al. (2012) Saccharomyces Genome Database: the genomics resource of budding yeast. Nucleic Acids Research 40(D1):D700-D705.

76. Schneider-Poetsch T, et al. (2010) Inhibition of eukaryotic translation elongation by cycloheximide and lactimidomycin. Nat Chem Biol 6(3):209-217. 
77. Lanthaler K, et al. (2011) Genome-wide assessment of the carriers involved in the cellular uptake of drugs: a model system in yeast. BMC Biology 9(1):70.

78. Limson MV \& Sweder KS (2010) Rapamycin Inhibits Yeast Nucleotide Excision Repair Independently of Tor Kinases. Toxicol. Sci. 113(1):77-84.

79. Katzmann DJ, Burnett PE, Golin J, Mahe Y, \& Moyerowley WS (1994) TRANSCRIPTIONAL CONTROL OF THE YEAST PDR5 GENE BY THE PDR3 GENE-PRODUCT. Molecular and Cellular Biology 14(7):4653-4661.

80. Owsianik G, Balzi L, \& Ghislain M (2002) Control of 26S proteasome expression by transcription factors regulating multidrug resistance in Saccharomyces cerevisiae. Molecular Microbiology 43(5):1295-1308.

81. Brôco N, Tenreiro S, Viegas CA, \& Sá-Correia I (1999) FLR1 gene (ORF YBR008c) is required for benomyl and methotrexate resistance in Saccharomyces cerevisiae and its benomyl-induced expression is dependent on Pdr3 transcriptional regulator. Yeast 15(15):1595-1608.

82. Parsons AB, et al. (2004) Integration of chemical-genetic and genetic interaction data links bioactive compounds to cellular target pathways. Nat Biotech 22(1):62-69.

83. Jonikas MC, et al. (2009) Comprehensive Characterization of Genes Required for Protein Folding in the Endoplasmic Reticulum. Science 323(5922):16931697.

84. Onda M, Ota K, Chiba T, Sakaki Y, \& Ito T (2004) Analysis of gene network regulating yeast multidrug resistance by artificial activation of transcription factors: involvement of Pdr3 in salt tolerance. Gene 332(0):51-59.

85. Workman CT, et al. (2006) A Systems Approach to Mapping DNA Damage Response Pathways. Science 312(5776):1054-1059. 
86. Teixeira MC, et al. (The YEASTRACT database: a tool for the analysis of transcription regulatory associations in Saccharomyces cerevisiae. Nucleic Acids Research 34(suppl 1):D446-D451.

87. Flynn PJ \& Reece RJ (1999) Activation of Transcription by Metabolic Intermediates of the Pyrimidine Biosynthetic Pathway. Mol. Cell. Biol. 19(1):882-888.

88. Brizzio V, Gammie AE, Nijbroek G, Michaelis S, \& Rose MD (1996) Cell fusion during yeast mating requires high levels of a-factor mating pheromone. The Journal of Cell Biology 135(6):1727-1739.

89. Devaux Fdr, Carvajal E, Moye-Rowley S, \& Jacq C (2002) Genome-wide studies on the nuclear PDR3-controlled response to mitochondrial dysfunction in yeast. Febs Letters 515(1â€"“3):25-28.

90. Devauz F, et al. (2001) An artificial transcription activator mimics the genomewide properties of the yeast Pdr1 transcription factor. Embo Reports 2(6):493498.

91. Hamel P, et al. (2004) Redundancy in the function of mitochondrial phosphate transport in Saccharomyces cerevisiae and Arabidopsis thaliana. Molecular Microbiology 51(2):307-317.

92. Guzder SN, Sommers CH, Prakash L, \& Prakash S (2006) Complex Formation with Damage Recognition Protein Rad14 Is Essential for Saccharomyces cerevisiae Rad1-Rad10 Nuclease To Perform Its Function in Nucleotide Excision Repair In Vivo. Molecular and Cellular Biology 26(3):1135-1141.

93. Wilcox LJ, et al. (2002) Transcriptional Profiling Identifies Two Members of the ATP-binding Cassette Transporter Superfamily Required for Sterol Uptake in Yeast. Journal of Biological Chemistry 277(36):32466-32472. 
94. Tong A, et al. (2004) Global mapping of the yeast genetic interaction network. Science 303:808 - 813.

95. Smets B, et al. (2010) Life in the midst of scarcity: adaptations to nutrient availability in Saccharomyces cerevisiae. Current Genetics 56(1):1-32.

96. De Virgilio C \& Loewith R (2006) The TOR signalling network from yeast to man. The International Journal of Biochemistry \&amp; Cell Biology 38(9):1476-1481.

97. Giannattasio S, Liu Z, Thornton J, \& Butow RA (2005) Retrograde Response to Mitochondrial Dysfunction Is Separable from TOR1/2 Regulation of Retrograde Gene Expression. Journal of Biological Chemistry 280(52):42528-42535.

98. De Virgilio C (2012) The essence of yeast quiescence. FEMS Microbiology Reviews 36(2):306-339.

99. Umekawa M \& Klionsky DJ (2012) Ksp1 Kinase Regulates Autophagy via the Target of Rapamycin Complex 1 (TORC1) Pathway. Journal of Biological Chemistry 287(20):16300-16310.

100. Inoki K, Ouyang H, Li Y, \& Guan K-L (2005) Signaling by Target of Rapamycin Proteins in Cell Growth Control. Microbiology and Molecular Biology Reviews 69(1):79-100.

101. Barz T, Ackermann K, \& Pyerin W (2003) Perturbation of protein kinase CK2 uncouples executive part of phosphate maintenance pathway from cyclin-CDK control. Febs Letters 537(1-3):210-214.

102. Reinke A, et al. (2004) TOR Complex 1 Includes a Novel Component, Tco89p (YPL180w), and Cooperates with Ssd1p to Maintain Cellular Integrity in Saccharomyces cerevisiae. Journal of Biological Chemistry 279(15):1475214762. 
103. Conibear E, Cleck JN, \& Stevens TH (2003) Vps51p Mediates the Association of the GARP (Vps52/53/54) Complex with the Late Golgi t-SNARE Tlg1p. Mol. Biol. Cell 14(4):1610-1623.

104. Conibear E \& Stevens TH (2000) Vps52p, Vps53p, and Vps54p Form a Novel Multisubunit Complex Required for Protein Sorting at the Yeast Late Golgi. Molecular Biology of the Cell 11(1):305-323.

105. Takagi K, et al. (2012) Involvement of Golgi-associated retrograde protein complex in the recycling of the putative Dnf aminophospholipid flippases in yeast. Biochemical and Biophysical Research Communications 417(1):490-494.

106. Hillenmeyer M, et al. (2010) Systematic analysis of genome-wide fitness data in yeast reveals novel gene function and drug action. Genome Biology 11(3):R30.

107. Søe R, et al. (2007) Sordarin Derivatives Induce a Novel Conformation of the Yeast Ribosome Translocation Factor eEF2. Journal of Biological Chemistry 282(1):657-666.

108. Cardenas ME, Cutler NS, Lorenz MC, Di Como CJ, \& Heitman J (1999) The TOR signaling cascade regulates gene expression in response to nutrients. Genes \& Development 13(24):3271-3279.

109. Fekete C, Posta K, \& Hornok L (2001) Primary structure and transcription patterns of RPL36, a ribosomal protein-encoding gene of the mycoparasitic fungus, Trichoderma hamatum. Current Genetics 39(3):183-189.

110. Schneider-Poetsch T, Usui T, Kaida D, \& Yoshida M (2010) Garbled messages and corrupted translations. Nat Chem Biol 6(3):189-198.

111. Francisco-Velilla R \& Remacha M (2010) In vivo formation of a stable pentameric (P2 alpha/P1 beta)-P0-(P1 alpha/P2 beta) ribosomal stalk complex in Saccharomyces cerevisiae. Yeast 27(9):693-704. 
112. Ballesta JPG, et al. (1999) Phosphorylation of the yeast ribosomal stalk. Functional effects and enzymes involved in the process. FEMS Microbiology Reviews 23(5):537-550.

113. Diaconu M, et al. (2005) Structural Basis for the Function of the Ribosomal L7/12 Stalk in Factor Binding and GTPase Activation. Cell 121(7):991-1004.

114. Garcia-Barrio M, Dong J, Ufano S, \& Hinnebusch AG (2000) Association of GCN1-GCN20 regulatory complex with the N-terminus of eIF2[alpha] kinase GCN2 is required for GCN2 activation. EMBO J 19(8):1887-1899.

115. Kim S \& Coulombe PA (2010) Emerging role for the cytoskeleton as an organizer and regulator of translation. Nat Rev Mol Cell Biol 11(1):75-81.

116. Hillenmeyer ME, et al. (2008) The Chemical Genomic Portrait of Yeast: Uncovering a Phenotype for All Genes. Science 320(5874):362-365.

117. Reid RJD, Kauh EA, \& Bjornsti M-A (1997) Camptothecin Sensitivity Is Mediated by the Pleiotropic Drug Resistance Network in Yeast. Journal of Biological Chemistry 272(18):12091-12099.

118. Gullo VP, McAlpine J, Lam KS, Baker D, \& Petersen F (2006) Drug discovery from natural products. J. Ind. Microbiol. Biotechnol. 33(7):523-531.

119. Haefner B (2003) Drugs from the deep: marine natural products as drug candidates. Drug Discovery Today 8(12):536-544.

120. Fürstner A, et al. (2007) Total Syntheses of the Actin-Binding Macrolides Latrunculin A, B, C, M, S and 16-epi-Latrunculin B. Chemistry - A European Journal 13(1):115-134.

121. Coué M, Brenner SL, Spector I, \& Korn ED (1987) Inhibition of actin polymerization by latrunculin A. Febs Letters 213(2):316-318. 
122. Ayscough KR, et al. (1997) High Rates of Actin Filament Turnover in Budding Yeast and Roles for Actin in Establishment and Maintenance of Cell Polarity Revealed Using the Actin Inhibitor Latrunculin-A. The Journal of Cell Biology 137(2):399-416.

123. Fuchs E \& Karakesisoglou I (2001) Bridging cytoskeletal intersections. Genes \& Development 15(1):1-14.

124. Brown SS (1999) Cooperation between microtubule- and actin-based motor proteins. Annu. Rev. Cell Dev. Biol. 15:63-80.

125. Spector I, Shochet N, Kashman Y, \& Groweiss A (1983) Latrunculins: novel marine toxins that disrupt microfilament organization in cultured cells. Science 219(4584):493-495.

126. Winsor B \& Schiebel E (1997) Review: An Overview of the Saccharomyces cerevisiae Microtubule and Microfilament Cytoskeleton. Yeast 13(5):399-434.

127. Evangelista M, Zigmond S, \& Boone C (2003) Formins: signaling effectors for assembly and polarization of actin filaments. Journal of Cell Science 116(13):2603-2611.

128. Smith AM, et al. (2009) Quantitative phenotyping via deep barcode sequencing. Genome Research 19(10):1836-1842.

129. Xu T, et al. (2011) The Marine Sponge-Derived Polyketide Endoperoxide Plakortide F Acid Mediates Its Antifungal Activity by Interfering with Calcium Homeostasis. Antimicrobial Agents and Chemotherapy 55(4):1611-1621.

130. Feng Y, et al. (2010) Antitrypanosomal Cyclic Polyketide Peroxides from the Australian Marine Sponge Plakortis sp. Journal of Natural Products 73(4):716719. 
131. Dembitsky VM (2008) Bioactive peroxides as potential therapeutic agents. European Journal of Medicinal Chemistry 43(2):223-251.

132. Patil AD, Freyer AJ, Carte B, Johnson RK, \& Lahouratate P (1996) Plakortides, Novel Cyclic Peroxides from the Sponge Plakortis halichondrioides: Activators of Cardiac SR-CA2+-Pumping ATPase. Journal of Natural Products 59(3):219223.

133. Li X, et al. (2011) Regulating Cytoplasmic Calcium Homeostasis Can Reduce Aluminum Toxicity in Yeast. Plos One 6(6).

134. Popa C-V, Dumitru I, Ruta LL, Danet AF, \& Farcasanu IC (2010) Exogenous oxidative stress induces $\mathrm{Ca} 2+$ release in the yeast Saccharomyces cerevisiae. FEBS Journal 277(19):4027-4038.

135. Cunningham KW (2011) Acidic calcium stores of Saccharomyces cerevisiae. Cell Calcium 50(2):129-138.

136. Pizzo P \& Pozzan T (2007) Mitochondria endoplasmic reticulum choreography: structure and signaling dynamics. Trends in cell biology 17(10):511-517.

137. Cyert MS (2003) Calcineurin signaling in Saccharomyces cerevisiae: how yeast go crazy in response to stress. Biochemical and Biophysical Research Communications 311(4):1143-1150.

138. Perrone GG, Tan S-X, \& Dawes IW (2008) Reactive oxygen species and yeast apoptosis. Biochimica Et Biophysica Acta-Molecular Cell Research 1783(7):1354-1368.

139. Carmona-Gutierrez D, et al. (2010) Apoptosis in yeast: triggers, pathways, subroutines. Cell Death Differ 17(5):763-773. 
140. Jamieson DJ, Rivers SL, \& Stephen DWS (1994) Analysis of Saccharomyces cerevisiae proteins induced by peroxide and superoxide stress. Microbiology 140(12):3277-3283.

141. Estruch F (2000) Stress-controlled transcription factors, stress-induced genes and stress tolerance in budding yeast. FEMS Microbiology Reviews 24(4):469486.

142. Veal EA, Ross SJ, Malakasi P, Peacock E, \& Morgan BA (2003) Ybp1 Is Required for the Hydrogen Peroxide-induced Oxidation of the Yap1 Transcription Factor. Journal of Biological Chemistry 278(33):30896-30904.

143. Spitzer M, et al. (2011) Cross-species discovery of syncretic drug combinations that potentiate the antifungal fluconazole. Mol Syst Biol 7.

144. Yokoyama H, et al. (2006) Involvement of calcineurin-dependent degradation of Yap1p in $\mathrm{Ca} 2+-$ induced $\mathrm{G}(2)$ cell-cycle regulation in Saccharomyces cerevisiae. Embo Reports 7(5):519-524.

145. Takatsume Y, et al. (2010) Calcineurin/Crz1 destabilizes Msn2 and Msn4 in the nucleus in response to $\mathrm{Ca} 2+$ in Saccharomyces cerevisiae. Biochemical Journal 427:275-287.

146. MacDiarmid CW, Gaither LA, \& Eide D (2000) Zinc transporters that regulate vacuolar zinc storage in Saccharomyces cerevisiae. Embo Journal 19(12):28452855.

147. Barber JMEK (2012) Chemical and Biological Aspects of Secondary Metabolites from Tongan Marine Sponges. PhD Thesis (Victoria University of Wellington).

148. Wang Y, et al. (2012) PubChem's BioAssay Database. Nucleic Acids Research 40(D1):D400-D412. 
149. Best H \& Miller J (2011) The Molecular Mechanism of the Microtubule Targeting Drug Laulimalide in Saccharomyces cerevisiae. Honours (Victoria University of Wellington, Wellington).

150. Gupta SS, et al. (2003) Antifungal Activity of Amiodarone Is Mediated by Disruption of Calcium Homeostasis. Journal of Biological Chemistry 278(31):28831-28839.

151. Jorgensen P, et al. (2002) High-Resolution Genetic Mapping With Ordered Arrays of Saccharomyces cerevisiae Deletion Mutants. Genetics 162(3):10911099.

152. Yibmantasiri P, et al. (2012) Molecular basis for fungicidal action of neothyonidioside, a triterpene glycoside from the sea cucumber, Australostichopus mollis. Molecular BioSystems 8(3):902-912.

153. Qiao W, Mooney M, Bird AJ, Winge DR, \& Eide DJ (2006) Zinc binding to a regulatory zinc-sensing domain monitored in vivo by using FRET. Proceedings of the National Academy of Sciences 103(23):8674-8679.

154. Bonilla M, Nastase KK, \& Cunningham KW (2002) Essential role of calcineurin in response to endoplasmic reticulum stress. Embo Journal 21(10):2343-2353.

155. Keith CT, Borisy AA, \& Stockwell BR (2005) Multicomponent therapeutics for networked systems. Nat Rev Drug Discov 4(1):71-78. 